for the award of the degree

"Doctor rerum naturalium"

of the Georg-August-Universität Göttingen

\title{
DEVELOPMENT AND APPLICATION OF CP-ENDOR SPECTROSCOPY ON PROTEIN RADICALS
}

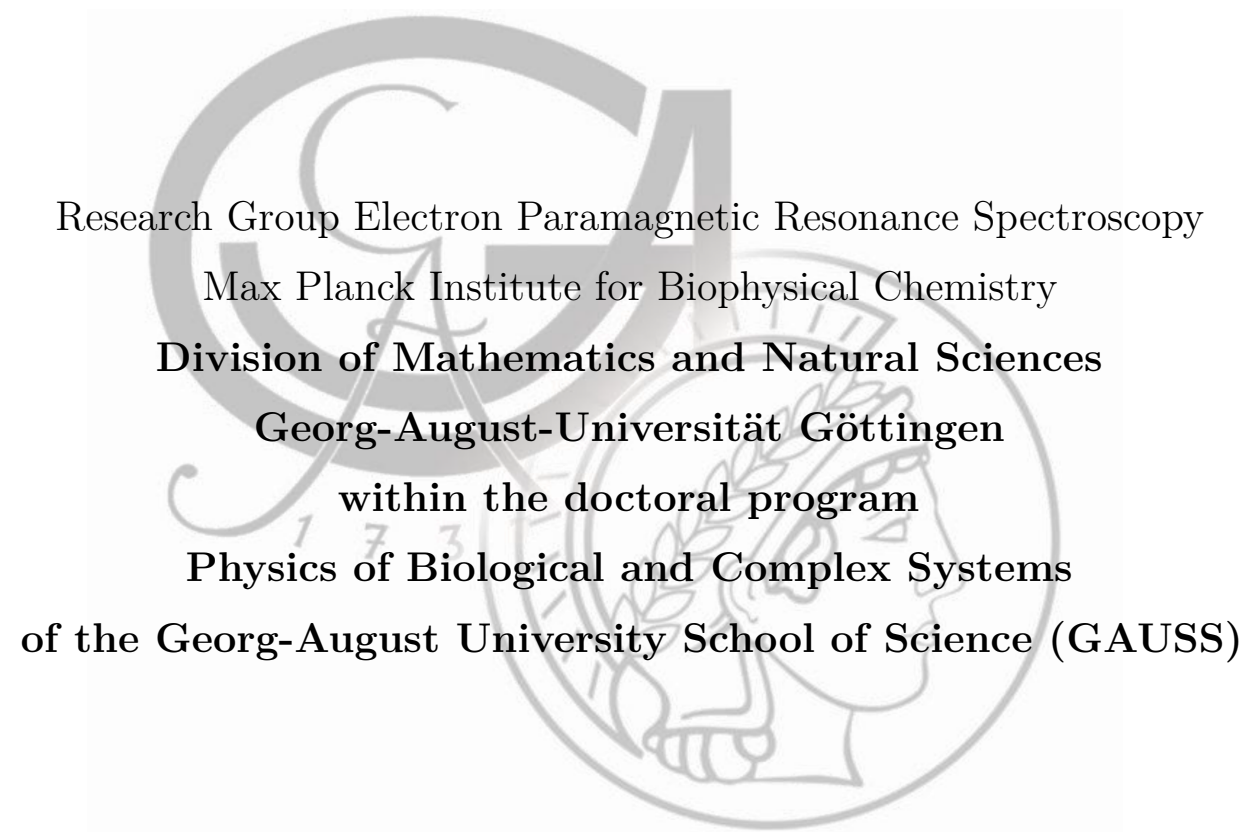

submitted by

Isabel Bejenke

from Kassel 



\section{Members of the $\mathrm{PhD}$ examination committee}

\section{Prof. Dr. Marina Bennati}

Member of the PhD thesis committee and 1st reviewer

Research Group Electron Paramagnetic Resonance Spectroscopy

Max Planck Institute for Biophysical Chemistry and

Institute for Organic and Biomolecular Chemistry

Georg-August-Universität Göttingen

\section{Prof. Dr. Christian Griesinger}

Member of the $\mathrm{PhD}$ thesis committee and 2nd reviewer

Department of NMR-based Structural Biology

Max Planck Institute for Biophysical Chemistry - Göttingen

\section{Prof. Dr. Martin Suhm}

Member of the PhD thesis committee

Physical Chemistry II

Institute for Physical Chemistry

Georg-August-Universität Göttingen

Prof. Dr. Sarah Köster

Institute for X-Ray Physics

Georg-August-Universität Göttingen

\section{Prof. Dr. Kai Tittmann}

Department of Molecular Enzymology

Schwann-Schleiden-Forschungszentrum

Georg-August-Universität Göttingen

\section{Prof. Dr. Martin Uecker}

Institute for Diagnostic and Interventional Radiology

University Medical Center - Göttingen 



\begin{abstract}
\end{abstract}
Electron-nuclear double resonance (ENDOR) spectroscopy is the method of choice for detecting magnetic nuclei in biomolecules which contain an unpaired electron spin. However, due to its low gyromagnetic ratio, the detection of deuterium couplings is a particular challenge for ENDOR spectroscopy. The standard ENDOR sequences suffer from either low sensitivity or line shape distortions. Yet, deuterium nuclei are among the most interesting targets for ENDOR spectroscopy. Hydrogen-bond environments of biomolecules can be investigated at the molecular scale by ${ }^{2} \mathrm{H}$ ENDOR spectroscopy in combination with $\mathrm{H}_{2} \mathrm{O} \rightarrow \mathrm{D}_{2} \mathrm{O}$ buffer exchange. Thus, aiming at improved sensitivity and/or spectral resolution, alternatives to the conventional ENDOR sequences have been proposed.

The cross-polarization edited ENDOR approach (CP-ENDOR) merges electron-nuclear cross polarization with ENDOR spectroscopy and generates an alternative polarization transfer scheme for it. In this thesis, the capability of the CP-ENDOR sequence to detect small hyperfine couplings between an electron spin and deuterium nuclei with high sensitivity is demonstrated at $94 \mathrm{GHz} / 3.4 \mathrm{~T}$.

The CP-ENDOR polarization transfer mechanism involving deuterium nuclei was established from single crystal studies of a deuterated malonic acid radical. The matching conditions for cross polarization and the CP-ENDOR intensities were determined experimentally and validated from analytical and numerical predictions.

The sensitivity for detecting small hyperfine couplings in CP-ENDOR is significantly improved when omitting the initial $\pi / 2$ pulse of the sequence. The modified sequence has been named "without preparation pulse" (WOP) CP-ENDOR. Its performance was evaluated on the EPR standard organic radical ${ }^{2} \mathrm{H}-\mathrm{BDPA}$ in crystalline powder form and resulted in an improvement of the signal-to-noise ratio by a factor of approximately five 
in comparison to the standard CP-ENDOR. The enhancement is attributed to different excitation profiles of the CP-ENDOR sequences, causing a central blind spot of different width in the ENDOR spectra. The width of the central blind spot of WOP CP-ENDOR is determined from analytical and numerical calculations and scales with the strength of the applied microwave irradiation pulse during the CP-step of the sequence.

Representative studies of the radical intermediate $\mathrm{ND}_{2} \mathrm{Y}_{731}{ }^{\bullet}$ in $E$. coli RNR illustrate the advantages of WOP CP-ENDOR in terms of signal-to-noise ratio and line shape in comparison to the well-established ENDOR sequences. Particularly, the WOP CPENDOR sequence has unmasked hyperfine tensor features of the amino group which are usually distorted by Mims blind spots in the ENDOR spectrum recorded with the wellestablished Mims ENDOR sequence. These advantages have allowed the establishment of the planarity of the functional group. 


\section{Contents}

1. EPR hyperfine spectroscopy of biomolecules . . . . . . . . . . 1

2. Introduction and theoretical background . . . . . . . . . 5

2.1. EPR spectroscopy . . . . . . . . . . . . . . . . 5

2.1.1. Spin Hamiltonian . . . . . . . . . . . . . . . . . 5

2.1.1.1. Electron and nuclear Zeeman interaction . . . . . . . 6

2.1.1.2. Hyperfine interaction . . . . . . . . . . . 7

2.1.1.3. Nuclear quadrupole interaction . . . . . . . . . . . . 9

2.2. Time evolution of spin systems . . . . . . . . . . . . . . . 9 9

2.2.1. Density operator formalism . . . . . . . . . . . . . . 10

2.3. Detection of hyperfine interactions via pulsed ENDOR spectroscopy . . 12

2.3.1. Analytically derived nuclear transition frequencies . . . . . . . . . 12

2.3.2. Davies ENDOR . . . . . . . . . . . . . . . . 15

2.3.3. Mims ENDOR . . . . . . . . . . . . . . . . . 20

2.3.4. High-field ENDOR spectroscopy . . . . . . . . . . . . . 23

2.3.5. Nuclear saturation in ENDOR spectroscopy . . . . . . . . . . . 25

2.4. Cross-polarization edited ENDOR spectroscopy . . . . . . . . . . . . . . 27

2.4.1. CP matching conditions from ${ }^{1} \mathrm{H}$ eNCP experiments . . . . . . . 29

2.4.2. ${ }^{1} \mathrm{H}$ CP-ENDOR spectroscopy . . . . . . . . . . . . . . 33

2.5. E.coli ribonucleotide reductase . . . . . . . . . . . . . . . . . . . . 38

3. Structure and scope of the thesis . . . . . . . . . . . . . 43

4. Materials and methods . . . . . . . . . . . . . . 45

4.1. Sample preparation . . . . . . . . . . . . . . . 45

4.1.1. Deuterated malonic acid radical of a $\gamma$-irradiated single crystal. . 45

4.1.2. Protonated BDPA radical/ Perdeuterated BDPA radical . . . . . 46 
4.1.3. Spin trapping of $\mathrm{ND}_{2} \mathrm{Y}_{731}$ in E. coli RNR . . . . . . . . . . . . . 47

4.2. Experimental setup . . . . . . . . . . . . . . . . . . . 48

4.2.1. ESE-detected field sweep experiments . . . . . . . . . . . . . 48

4.2.2. Rabi-nutations . . . . . . . . . . . . . . . . . . . 48

4.2.3. eNCP experiments . . . . . . . . . . . . . . . . 49

4.2.4. ENDOR experiments . . . . . . . . . . . . . . 50

4.2.4.1. CP-ENDOR . . . . . . . . . . . . . 50

4.2.4.2. Davies ENDOR . . . . . . . . . . . . . 51

4.2.4.3. Mims ENDOR . . . . . . . . . . . . . . . . . . . 52

4.3. Numerical simulations . . . . . . . . . . . . . . . . . . 52

4.3.1. Single crystal simulations . . . . . . . . . . . . . . . 53

4.3.1.1. eNCP simulation . . . . . . . . . . . 54

4.3.1.2. CP-ENDOR simulation . . . . . . . . . . . 56

4.3.1.3. Efficient simulation of the ENDOR step . . . . . . . . . 57

4.3.1.4. Heuristic simulation of the effect of $B_{1}$ inhomogeneity and relaxation during the $\mathrm{CP}$ and free evolution step . . . . . 58

4.3.2. Powder pattern simulations of ${ }^{2} \mathrm{H}-\mathrm{BDPA}$. . . . . . . . . . 58

4.3.2.1. Simulation strategy for powder pattern samples $-{ }^{2} \mathrm{H}-\mathrm{BDPA}$ simulations . . . . . . . . . . . . . . . . . . . 58

4.3.3. Powder pattern simulations of $\mathrm{ND}_{2} \mathrm{Y}_{731}$. . . . . . . . . . . 61

5. ${ }^{2} \mathrm{H}$ eNCP and ${ }^{2} \mathrm{H}$ CP-ENDOR single crystal studies of a deuterated malonic acid radical . . . . . . . . . . . . . . . . 63

5.1. The $S=1 / 2, I=1$ spin system . . . . . . . . . . . . 63

5.2. eNCP experiments and matching conditions for $S=1 / 2, I=1$. . . . 66

5.2.1. eNCP experiments . . . . . . . . . . . . . 68

5.2.2. Matching conditions from analytical treatment . . . . . . . 68

5.2.3. Matching conditions from numerical simulations . . . . . . . . 76

5.3. CP-ENDOR experiments and calculations of CP-ENDOR intensities . . 76

5.3.1. CP-ENDOR experiments . . . . . . . . . . . . 77

5.3.2. CP-ENDOR intensities from analytical treatment . . . . . . . . . 79

5.3.3. CP-ENDOR intensities from numerical treatment . . . . . . . . . 85

5.4. Discussion of eNCP and CP-ENDOR results . . . . . . . . . . . . . 86

5.4.1. ${ }^{2} \mathrm{H}$ eNCP investigations on a deuterated single crystal of malonic acid 86 
5.4.2. ${ }^{2} \mathrm{H}$ CP-ENDOR investigations on a deuterated single crystal of malonic acid . . . . . . . . . . . . . . . . . 87

6. Without preparation pulse (WOP) CP-ENDOR spectroscopy . . . 91

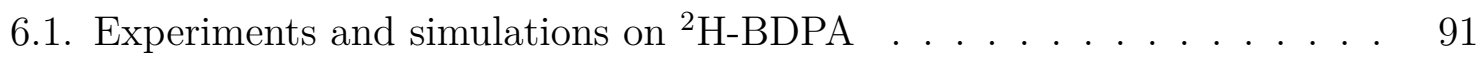

6.2. Microwave amplitude during spinlock - Numerical predictions and experimental trade-off . . . . . . . . . . . . . . . . . . . . . . . . . . . . . . . 98

6.3. Central blind spot in CP-ENDOR spectra derived from numerical and analytical excitation profiles . . . . . . . . . . . . . . . . . 104

6.3.1. Numerically derived excitation profiles of CP-ENDOR . . . . . . 104

6.3.2. Analytically derived excitation profile of WOP CP-ENDOR . . . 108

6.3.3. Experimentally observed central blind spot in WOP CP-ENDOR 111

6.4. Discussion of WOP CP-ENDOR investigations . . . . . . . . . . . . . 112

7. ${ }^{2} \mathrm{H}$ WOP CP-ENDOR of $\mathrm{ND}_{2} \mathrm{Y}_{731}{ }^{\bullet}$ from $E$. coli RNR . . . . . . 117

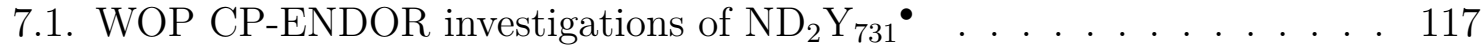

7.1.1. WOP CP-ENDOR performance . . . . . . . . . . . . . 118

7.1.2. Determination of the $\mathrm{ND}_{2}$-conformation from WOP CP-ENDOR 121

$\begin{array}{ll}\text { 7.1.3. Simulation of orientation-selective 2D WOP CP-ENDOR spectra } 127 & 12\end{array}$

7.2. Discussion of WOP CP-ENDOR on $\mathrm{ND}_{2} \mathrm{Y}_{731}$. . . . . . . . . . . . 132

7.2.1. WOP CP-ENDOR performance and orientation-selective CP-ENDOR simulations . . . . . . . . . . . . . . . . . 132

7.2.2. WOP CP-ENDOR for $\mathrm{ND}_{2}$-conformation studies . . . . . . . . . 136

8. Conclusions and perspectives for CP-ENDOR spectroscopy . . . . 139

Appendix . . . . . . . . . . . . . . . . . . . . 143

A. Davies ENDOR on a single crystal of a malonic acid radical . . . . . . . 143

B. Spin operator definitions . . . . . . . . . . . . . . . . . . . 144

B.1. Electron spin coupled to one nuclear spin-1 . . . . . . . . . . . . 144

B.2. Electron spin coupled to two nuclear spins-1 . . . . . . . . . . . . 145

B.3. Electron spin coupled to three nuclear spins-1 . . . . . . . . . . . 145

C. Transformation of spin operators into a new basis . . . . . . . . . . 146

D. Analytically calculated CP-ENDOR intensities for EPR $\gamma$ and EPR $\beta$ of the malonic acid single crystal . . . . . . . . . . . . . . . . . . . . . 148

D.1. Analytically calculated CP-ENDOR intensities for EPR $\gamma$ excitation 148

D.2. Analytically calculated CP-ENDOR intensities for EPR $\beta$ excitation 150

E. Control CP-ENDOR spectrum of ${ }^{2} \mathrm{H}-\mathrm{BDPA}$. . . . . . . . . . . . . . . 153 
F. PulseSPEL script for ${ }^{2} \mathrm{H}-\mathrm{BDPA}$ 2D CP-ENDOR experiments . . . . . . 154

G. Calculation script for plotting of analytically derived excitation profile . 157

H. eNCP spectrum of $\mathrm{ND}_{2} \mathrm{Y}_{731}{ }^{\circ} \ldots \ldots \ldots \ldots \ldots \ldots$

Bibliography . . . . . . . . . . . . . . . . . . . 161

Acknowledgments . . . . . . . . . . . . . . . . . . 173

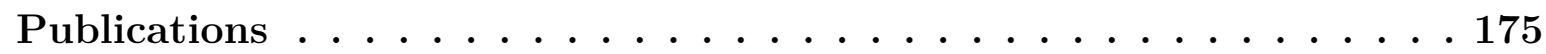




\section{List of abbreviations}

E. coli

ATP

BDPA

CDP

CP-ENDOR

DFT

DNP

ELDOR

EPR

ESE

FWHM

HFA

hfc

$\mathrm{HH}$

HTA

hta

lab

mw

NMR

NOVEL

NRMSD

PCET

\section{Escherichia coli}

Adenosine triphosphate

$\alpha, \gamma$,-bisdiphenylene- $\beta$-phenylallyl

Cytidine diphosphate

Cross-polarization edited ENDOR

Density function theory

Dynamic nuclear polarization

Electron-electron double resonance

Electron paramagnetic resonance

Electron spin echo

Full width at half maximum

High field approximation

Hyperfine coupling

Hartmann-Hahn

High temperature approximation

High-turning-angle

Laboratory

Microwave

Nuclear magnetic resonance

Nuclear spin orientation via electron spin locking

Normalized root-mean-square deviation

Proton-coupled electron transfer 


$\begin{array}{ll}\text { PDB ID } & \text { Protein database identification code } \\ \text { quad } & \text { Quadrupole } \\ \text { rf } & \text { Radio frequency } \\ \text { RNR } & \text { Ribonucleotide reductase } \\ \text { rt } & \text { Radical transfer } \\ \text { S/N } & \text { Signal-to-noise ratio } \\ \text { WOP } & \text { Without preparation pulse } \\ \text { Y } & \text { Tyrosine }\end{array}$




\section{List of figures}

2.1. Orientation of the magnetic field vector with respect to the $g$-tensor. . 6

2.2. EPR and NMR transitions illustrated in the first-order energy diagram 14

2.3. Davies ENDOR pulse sequence and population transfer scheme . . . . 16

2.4. Illustration of hole burning in Davies ENDOR . . . . . . . . . . . . . . 19

2.5. Pulse sequence for Mims ENDOR spectroscopy . . . . . . . . . . . . 21

2.6. Representative ESE-detected field sweep spectra of a tyrosyl radical at $9 \mathrm{GHz}$ and $94 \mathrm{GHz} . \ldots \ldots . . \ldots . . \ldots 24$

2.7. Polarization pattern between two Davies ENDOR sequences repeated for signal averaging . . . . . . . . . . . . . . . . 26

2.8. Illustration of the target nuclei type in ENDOR and DNP experiments 28

2.9. eNCP pulse sequence and idealized eNCP spectrum . . . . . . . . . . . 30

2.10. Electron- and nuclear-spin effective fields during the eNCP transfer . . 31

2.11. Illustration of a powder pattern eNCP spectrum . . . . . . . . . . . 33

2.12. CP-ENDOR pulse sequence, polarization transfer scheme and idealized CP-ENDOR spectrum . . . . . . . . . . . . . . . 34

2.13. Illustration of polarization transfer for a non-matched CP-ENDOR case 35

2.14. CP-ENDOR sequence and Davies ENDOR sequence described by the product operator formalism . . . . . . . . . . . . . . . . . . 36

2.15. Docking model of the E. coli $\alpha 2 \beta 2$ complex . . . . . . . . . . . . . . . 39

2.16. Schematic thermodynamic landscape for the rt pathway in E. coli RNR 40

4.1. $\quad$ ESE-detected field sweep spectrum of $\mathrm{ND}_{2} \mathrm{Y}_{731} \bullet$ for radical yield determination . . . . . . . . . . . . . . . . 47

4.2. $\quad$ Pulse timing and pulse phases in Hahn-echo experiments . . . . . . . . 48 
4.3. $\quad$ Electron-spin and nuclear-spin Rabi-nutation sequences . . . . . . . . . 49

4.4. Pulse timing and pulse phases in eNCP experiments . . . . . . . . . . 50

4.5. Pulse timing and pulse phases in CP-ENDOR experiments . . . . . . 50

4.6. Pulse timing and pulse phases in Davies ENDOR experiments . . . . . 52

4.7. Pulse timing and pulse phases in Mims ENDOR experiments . . . . . 52

4.8. Illustration of the eNCP simulation steps . . . . . . . . . . . . . 54

4.9. Illustration of the CP-ENDOR simulation steps . . . . . . . . . . 56

4.10. Illustration of the calculation strategy for powder pattern CP-ENDOR simulations . . . . . . . . . . . . . . . . 60

5.1. First-order energy diagram for an electron spin-1/2 coupled to a nuclear spin-1 . . . . . . . . . . . . . . . . 65

5.2. Chemical structure of malonic acid and ESE-detected field sweep spectrum 66

5.3. eNCP experiments and calculated matching conditions for a deuterated malonic acid single crystal . . . . . . . . . . . . . . 67

5.4. Illustration of the rotation angles used to transfer the Hamiltonian into the tilted frame . . . . . . . . . . . . . . . . . . 72

5.5. $\quad{ }^{2} \mathrm{H}$ CP-ENDOR on a deuterated malonic acid single crystal . . . . . . 78

5.6. First-order energy diagram for an electron spin-1/2 coupled to a nuclear spin-1 and negative hyperfine and quadrupole couplings . . . . . . . 83

5.7. Analytically calculated CP-ENDOR signal intensities for CP matched and CP non-matched cases . . . . . . . . . . . . . . . . 85

5.8. CP-ENDOR population transfer scheme for an electron spin coupled to a deuterium . . . . . . . . . . . . . . . . . 88

6.1. Illustration of the standard and WOP CP-ENDOR sequences . . . . . 92

6.2. Comparison of standard CP-ENDOR, WOP CP-ENDOR, Mims ENDOR

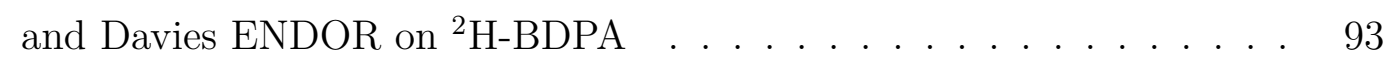

6.3. eNCP spectrum of ${ }^{2} \mathrm{H}-\mathrm{BDPA} \ldots \ldots \ldots . \ldots . \ldots . \ldots 94$

6.4. Standard CP-ENDOR and WOP CP-ENDOR experiments and simulations of ${ }^{2} \mathrm{H}-\mathrm{BDPA} \ldots \ldots . \ldots . \ldots . . \ldots 95$

6.5. Landscape and contour plots of 2D WOP CP-ENDOR and standard 2D

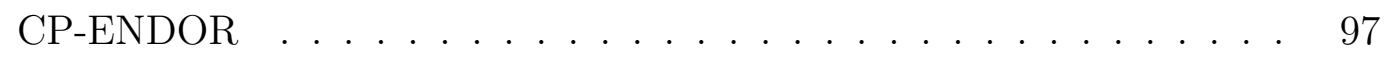

6.6. Slices from experimental 2D CP-ENDOR spectra . . . . . . . . . . 98 
6.7. Standard CP-ENDOR and WOP CP-ENDOR single crystal simulations for $S=1 / 2, I=1 \ldots \ldots \ldots$. . . . . . . . . . . . . . 99

6.8. Calculated CP-ENDOR signal intensity as a function of $A / 2 \pi$ at constant $\omega_{1 \mathrm{e}} / 2 \pi \ldots \ldots \ldots \ldots \ldots 1 . \ldots \ldots \ldots \ldots$

6.9. Calculated WOP CP-ENDOR intensity (non-matched) as a function of $\omega_{1 \mathrm{e}} / 2 \pi$ and $A / 2 \pi \ldots \ldots \ldots \ldots . \ldots \ldots 2 . \ldots \ldots$

6.10. WOP CP-ENDOR of ${ }^{2} \mathrm{H}-\mathrm{BDPA}$ with low $\omega_{1 \mathrm{e}} / 2 \pi \ldots$. . . . . . . . 103

6.11. Numerically calculated excitation profiles of spinlock ENDOR . . . . . 105

6.12. Numerically calculated CP-ENDOR excitation profiles . . . . . . . . . 107

6.13. Numerically calculated spinlock ENDOR excitation profile dependency on $\omega_{1 \mathrm{e}} / 2 \pi \ldots \ldots \ldots \ldots \ldots$

6.14. Electron spin offset dependency of the effective field illustrated for EPR $\beta 109$

6.15. Analytically calculated excitation profile of WOP CP-ENDOR . . . . . 110

6.16. Experimental hole function observed in spinlock WOP ENDOR on ${ }^{1} \mathrm{H}-$ BDPA . . . . . . . . . . . . . . . . . . . 111

6.17. Illustration of hole burning and central blind spot in Davies ENDOR and WOP CP-ENDOR spectroscopy . . . . . . . . . . . . . . . . . . 113

7.1. $\quad$ ENDOR performance comparison on $\mathrm{ND}_{2} \mathrm{Y}_{731}$. . . . . . . . . . . . . 119

7.2. Comparison of WOP CP-ENDOR and Mims ENDOR on $\mathrm{ND}_{2} \mathrm{Y}_{731}$. . 120

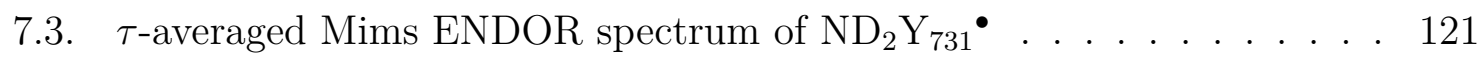

7.4. Landscape plots of orientation-selective 2D WOP CP-ENDOR experi-

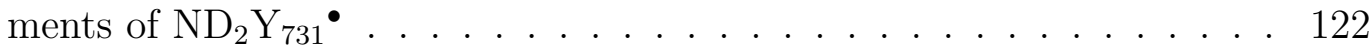

7.5. Orientation-selective WOP CP-ENDOR experiments and simulations of $\mathrm{ND}_{2} \mathrm{Y}_{731} \bullet \ldots \ldots \ldots \ldots \ldots \ldots \ldots$

7.6. Systematic ENDOR simulations for conformational changes of $\mathrm{ND}_{2}$. . 125

7.7. Orientation-selective 2D WOP CP-ENDOR experiments and simulations of $\mathrm{ND}_{2} \mathrm{Y}_{731} \bullet \ldots \ldots \ldots \ldots \ldots$

7.8. Comparison of experiments and two simulation approaches for orientationselective WOP CP-ENDOR of $\mathrm{ND}_{2} \mathrm{Y}_{731} \bullet \ldots \ldots$. . . . . . . . . . . . . . 129

7.9. Orientation-selective WOP CP-ENDOR and spinlock ENDOR simulation of $\mathrm{ND}_{2} \mathrm{Y}_{731} \bullet \ldots \ldots \ldots \ldots$. . . . . . . . . . . . . . 130

7.10. Systematic spinlock ENDOR simulations for conformational changes of $\mathrm{ND}_{2}$. 
7.11. Illustration of 2D CP-ENDOR summation and pseudo spinlock ENDOR summation in powder samples . . . . . . . . . . . . . . . . . . 134

A1. Davies ENDOR of a single crystal of deuterated malonic acid . . . . . 143

E2. Mims, Davies and WOP CP-ENDOR control experiments of ${ }^{2} \mathrm{H}-\mathrm{BDPA} 153$

H3. eNCP spectrum of $\mathrm{ND}_{2} \mathrm{Y}_{731}$. . . . . . . . . . . . . . . . 159 


\section{List of tables}

2.1. Analytically calculated CP-ENDOR intensities for an electron spin-1/2 coupled to a proton . . . . . . . . . . . . . . . . 35

5.1. Rotation angles for each EPR manifold . . . . . . . . . . . . . . . 73

5.2. Turning angles for the case of deuterated malonic acid . . . . . . . . . . 74

5.3. Analytically calculated matching conditions for the malonic acid case . $\quad 75$

5.4. Analytically calculated CP-ENDOR intensities for the malonic acid case 85

6.1. Hyperfine tensor values for the simulation of ${ }^{2} \mathrm{H}-\mathrm{BDPA} \quad \ldots . . . \quad . \quad . \quad 95$

7.1. Hyperfine tensor values, quadrupole tensor values and corresponding Euler angles for $\mathrm{ND}_{2} \mathrm{Y}_{731}{ }^{\circ} \ldots \ldots \ldots$. . . . . . . . . . . . . . . . . 124 



\section{EPR hyperfine spectroscopy of biomolecules}

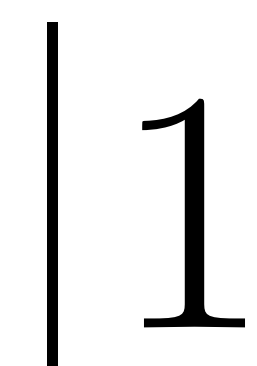

A key source of information for local structural investigations at the molecular scale is the interaction between electron spins and magnetic nuclei in close vicinity - the hyperfine interaction. Hyperfine interactions and the resulting hyperfine splittings arise in several spectroscopy techniques such as nuclear magnetic resonance and optical laser spectroscopy. However, these splittings are usually best resolved in electron paramagnetic resonance (EPR) spectroscopy.

EPR spectroscopy utilizes electromagnetic irradiation in the microwave range for gaining nanometer to sub-nanometer scale insights into systems containing at least one unpaired electron spin. The versatility of EPR techniques applied at different microwave frequencies/magnetic fields offers spectroscopists a wide range of possibilities to determine distances and molecular structures. These involve paramagnetic centers in crystalline and amorphous materials, as well as in frozen and liquid solutions. In particular, the capability to investigate electron spins occurring as highly reactive, thus transient, short-living intermediates in systems as complex as biomolecules, established EPR spectroscopy as a valuable biophysical technique. It provides structural information over a distance range from atomic scale up to $10 \mathrm{~nm}$, depending on which interaction of the electron spin is probed 1, 2. Several EPR hyperfine techniques can report on hyperfine interactions corresponding to distances between electron and nuclear spins up to about $2 \mathrm{~nm}$. However, most of these are restricted to application at low magnetic fields/frequencies ( $\leqslant 34 \mathrm{GHz} / 3.4 \mathrm{~T}$ ) for which nuclear resolution is limited.

The method of choice for high-resolution EPR hyperfine spectroscopy is referred to as electron-nuclear double resonance (ENDOR) spectroscopy 3 [5]. The technique relies on microwave and radio frequency irradiation and allows for the determination of hyperfine couplings of several megahertz with kilohertz resolution, unique among the available 
EPR hyperfine techniques. This resolution becomes particularly beneficial when nuclei with different magnetic moments are involved. Unlike EPR resonances, ENDOR lines of different nuclear spins appear at characteristic frequency ranges according to their respective nuclear Larmor frequencies. The separation of the latter increases with the increase of the fields/microwave frequencies. Indeed, the ENDOR method does not only report on amount and identity of the coupled nuclei, but also on their distance and spatial localization with respect to the paramagnetic center. Through the years, technical developments in high-field/high-frequency pulse EPR instrumentation have empowered ENDOR spectroscopy to spatially localize nuclei near the electron spin of several biomolecular systems ${ }^{[6]}$. For instance, ENDOR spectroscopy has played a key role in investigations of the molecular-level mechanism of H-cluster biosynthesis in $[\mathrm{FeFe}]$-hydrogenase ${ }^{[9]}[10]$, the electronic and geometric structure of photosystem II in its highest metastable catalytic state ${ }^{[11]}$ and the identification of stable as well as intermediate radicals in E. coli ribonucleotide reductases 12 15. For the latter, ENDOR spectroscopy has not only facilitated the identification of intermediates, but also allowed for conclusions on the enzyme's radical transfer mechanism from high-field/highfrequency ENDOR studies $\left(\geqslant 94 \mathrm{GHz} / 3.4 \mathrm{~T}\right.$ ) in combination with $\mathrm{H}_{2} \mathrm{O} \rightarrow \mathrm{D}_{2} \mathrm{O}$ solvent exchange $16[19$. Solvent exchange is an important approach for biomolecular ENDOR studies because buffer exchanged deuterium nuclei are probed at ENDOR frequency ranges well-separated from those of non-exchangeable protons of the biomolecule's backbone 2022 . In this way, local structural information at the molecular scale is gained. This forms the basis for the elucidation of hydrogen bond environments in biomolecules such as enzymes $16,23,24$. Hydrogen bonds in enzymes are important to understand since they are essential for stabilizing the biomolecule's structure(s) and/or mediating its biochemistry.

The deuterium nuclear spin, however, represents a challenging target for ENDOR spectroscopy. Indeed, the gyromagnetic ratio $\left(\gamma_{\mathrm{n}}\right)$ of deuterium is approximately 6.5 -times lower than that of a proton. This property inherently reduces the hyperfine coupling constants by the same factor.

For detecting small hyperfine couplings, the established ENDOR sequences 4 [4 5 are associated with either low sensitivity or line shape distortions. Additionally, in conventional ENDOR the electron and nuclear spins are manipulated on the timescale defined by the fast electron spin relaxation properties ( $\mu$ s to ms range) without considering the longer relaxation times of the nuclear spin (on the order of several seconds at low $T$ ). 
However, nuclear relaxation pathways are crucial for optimal ENDOR performance during long-term signal acquisition. The more the electron-spin and nuclear-spin relaxation properties differ, the more severe become nuclear saturation issues and resulting ENDOR sensitivity losses. The discrepancy in relaxation rates becomes particularly significant for low- $\gamma$ nuclei like deuterium and at low temperatures. This, however, is a standard experimental condition for ENDOR experiments of biomolecules. Unfortunately, nuclear-spin relaxation rates of specific nuclei nearby the paramagnetic center are difficult to access. They are expected to differ by at least an order of magnitude from those of the electron spin. Nonetheless, only ENDOR spectroscopy can provide high-resolution hyperfine spectra of small deuterium couplings.

Facing these difficulties, there is an ongoing effort to develop new methods aiming at resolution and/or sensitivity enhancement. This thesis investigates an alternative polarization transfer scheme using electron-nuclear cross polarization for ENDOR spectroscopy 25 . The resulting cross-polarization edited ENDOR sequence (CP-ENDOR) was developed only recently 25 and provides improved sensitivity and robustness towards nuclear saturation for detection of large proton hyperfine couplings $25 \sqrt[27]{27}$. Though the CP-ENDOR polarization transfer mechanism for protons has been published 25 , its extension and application to deuterium nuclei has not been reported yet. In contrast to the proton spin $(I=1 / 2)$, deuterium has a nuclear spin of $I=1$ which increases the number of energy levels potentially involved in the CP-process. Furthermore, the deuterium exhibits a quadrupolar moment resulting in an additional quadrupole coupling which potentially complicates the CP-ENDOR polarization transfer.

This thesis focuses on the development and application of $94 \mathrm{GHz} / 3.4 \mathrm{~T}^{2} \mathrm{H} \mathrm{CP-ENDOR}$ spectroscopy for the detection of small hyperfine interactions between the electron spin and deuterium nuclear spins. Firstly, the CP-ENDOR polarization transfer mechanism involving deuterium nuclei will be established from experiments and simulations on two representative radical systems. These include a single crystal of the deuterated malonic acid radical and the EPR standard organic radical ${ }^{2} \mathrm{H}-\mathrm{BDPA}$ in crystalline powder form. Secondly, a representative application of ${ }^{2} \mathrm{H}$ CP-ENDOR will be presented in which the structure of the amino group of an amino-tyrosyl radical intermediate in the E. coli ribonucleotide reductase is elucidated. 



\section{Introduction and theoretical background}

In this chapter a brief overview of state-of-the-art electron paramagnetic resonance (EPR) spectroscopy with special focus on the detection of hyperfine interactions via electron-nuclear double resonance spectroscopy is given. Further, the biological system E. coli ribonucleotide reductases (RNR) studied in this thesis is introduced.

\subsection{EPR spectroscopy}

This section features an overview of the theoretical framework relevant for the description of paramagnetic systems investigated in this thesis. It comprises fundamental spin Hamil-

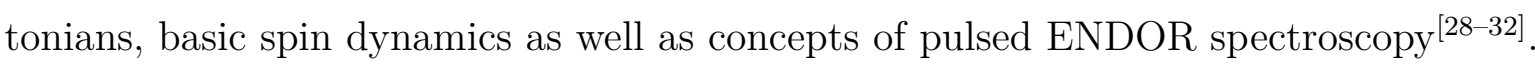

\subsubsection{Spin Hamiltonian}

The static Hamiltonian in energy units $\left(H_{0}\right)$ of an electron spin $S=1 / 2$ coupled to a nuclear spin $I$ (considering here only the terms relevant for the organic radicals studied in this thesis) is given by

$$
H_{0}=H_{\mathrm{EZ}}+H_{\mathrm{NZ}}+H_{\mathrm{HFC}}+H_{\mathrm{Q}}
$$

The terms of $H_{0}$ correspond to the electron Zeeman interaction $H_{\mathrm{EZ}}$, nuclear Zeeman interaction $H_{\mathrm{NZ}}$, electron-nuclear hyperfine interaction $H_{\mathrm{HFC}}$ and, if the spin system contains at least one nucleus with $I>1 / 2$, the quadrupole contribution $H_{\mathrm{Q}}$. 


\subsubsection{Electron and nuclear Zeeman interaction}

The basis for all EPR spectroscopy is the interaction between an unpaired electron spin and the external magnetic field, described by the electron Zeeman term

$$
H_{\mathrm{EZ}}=\mu_{\mathrm{B}} \vec{B}_{0}^{\mathrm{T}} g \hat{S}
$$

where $\mu_{\mathrm{B}}$ is the Bohr magneton, $\overrightarrow{B_{0}}$ the external static magnetic field vector, $g$ the $g$-tensor and $\hat{S}$ the electron spin vector operator ${ }^{1}$. In the principle axis system of $g$, its principle values are indicated by $g_{x}, g_{y}$ and $g_{z}$ and three so-called Euler angles $(\alpha, \beta, \gamma)$ define the orientation of the principle axis system in the molecular axis system. Usually, the $g$-principle axis frame is chosen to coincide with the molecular frame and all other interaction tensors (i.e. hfc-tensor) are referred to this frame. However, since the EPR spectrum of a powder or frozen solution will depend on the relative orientation of the applied magnetic field with respect to the molecular axis system, this angular dependence results in an EPR resonance condition

$$
\Delta E=h \nu=\mu_{\mathrm{B}} \vec{B}_{0}^{\mathrm{T}} g(\phi, \theta)
$$

where $\theta$ and $\phi$ are the polar angles of the applied field within the molecular $g$-tensor principal axis system as illustrated in Fig. 2.1.

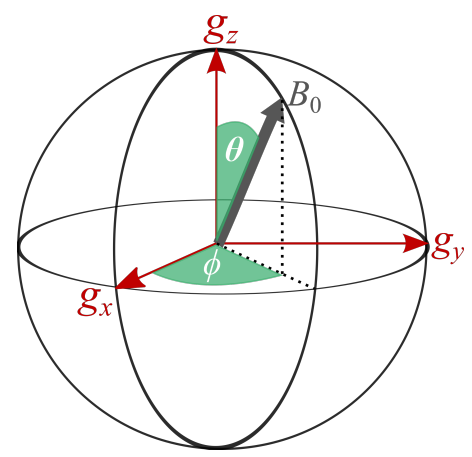

Figure 2.1: Orientation of the magnetic field vector with respect to the $\boldsymbol{g}$-tensor. The polar angles $\theta$ and $\phi$ define the orientation of the magnetic field vector $B_{0}$ in the $g$-tensor principal axis frame. Figure adapted from 30 .

From this, the effective $g_{\text {eff }}$ value can be expressed according to 33

$$
g_{\mathrm{eff}}(\theta, \phi)=\sqrt{g_{x}^{2} \sin ^{2} \theta \cos ^{2} \phi+g_{y}^{2} \sin ^{2} \theta \sin ^{2} \phi+g_{z}^{2} \cos ^{2} \theta}
$$

\footnotetext{
${ }^{1}$ The notation in this thesis uses $\vec{A}$ to refer to a vector, $A$ to refer to a tensor or matrix and $\hat{A}$ to refer
} to a vector operator. The superscript ${ }^{\mathrm{T}}$ indicates the respective transpose of a vector or tensor. 
which gives the effective $g_{\text {eff }}$ value in polar coordinates relative to the $g$-tensor principle axis coordinate system.

Consequently, each combination of $\theta$ and $\phi$ refers to one particular orientation of $\overrightarrow{B_{0}}$ in the $g$-tensor frame and the so-called powder pattern observed from samples of multiple molecules randomly oriented with respect to the external magnetic field. This is considered in powder pattern calculations by averaging over a number of $N$ combinations of $\theta$ and $\phi\left(N_{\theta}\right.$ and $\left.N_{\phi}\right)$.

In absence of cubic symmetry $\left(g_{x}=g_{y}=g_{z}\right)$ the tensor is either called axial symmetric $\left(g_{x}=g_{y} \neq g_{z}\right)$ or rhombic $\left(g_{x} \neq g_{y} \neq g_{z}\right)$. Deviations of the $g$-tensor values from the free electron spin value $g_{\mathrm{e}}$ are majorly caused by spin-orbit coupling which mixes orbital angular momentum into the spin ground state.

Analogous to the electron spin, also the nuclear Zeeman interaction refers to the interaction between the nuclear spin and the external static magnetic field:

$$
H_{\mathrm{NZ}}=-\mu_{\mathrm{N}} g_{\mathrm{n}} \vec{B}_{0}^{\mathrm{T}} \hat{I}
$$

where $\mu_{\mathrm{N}}$ is the nuclear magneton, $g_{\mathrm{n}}$ the nuclear $g$ value considered to be unresolved and thus isotropic, and $\hat{I}$ the nuclear spin vector operator. Depending on nucleus and isotope, $g_{\mathrm{n}}$ can be positive or negative. In general, the nuclear Zeeman contribution is much weaker than the electron Zeeman contribution which will be discussed later. For instance for protons it is only 1/660 of the electron spin interaction and for all other nuclei even less 30$]$.

\subsubsection{Hyperfine interaction}

The interaction in focus of this thesis and important information source in EPR spectroscopy is the hyperfine interaction between electron and nuclear spins defined by the Hamiltonian

$$
H_{\mathrm{HFC}}=\hat{S} A \hat{I}
$$

where $A$ is the hyperfine tensor. In the principle axis system of $A$, its principle values are described by $A_{x}, A_{y}$ and $A_{z}$ and three Euler angles $(\alpha, \beta, \gamma)$. These angels are defining the orientation of the principle axis system in the molecular axis system or $g$-tensor principle axis system. The hyperfine tensor comprises two terms, referring to the isotropic Fermi contact interaction $H_{\mathrm{F}}$ and the electron-nuclear dipole-dipole 
coupling $H_{\mathrm{DD}}$. The ladder is an anisotropic coupling given by 29

$$
H_{\mathrm{DD}}=\frac{\mu_{0}}{4 \pi} g_{\mathrm{e}} \mu_{\mathrm{B}} g_{\mathrm{n}} \mu_{\mathrm{N}}\left[\frac{3\left(\hat{S}^{\mathrm{T}} \vec{r}\right)\left(\vec{r}^{\mathrm{T}} \hat{I}\right)}{\mathrm{r}^{5}}-\frac{\hat{S}^{\mathrm{T}} \hat{I}}{\mathrm{r}^{3}}\right]
$$

where $\mathrm{r}$ is the distance between the two spins (in length unit assuming electron and nucleus as point dipoles) connected by the vector $\vec{r}$. $H_{\mathrm{DD}}$ is usually expressed considering electron spin delocalization, thus after integration over the spatial electron spin distribution it is obtained as

$$
H_{\mathrm{DD}}=\hbar \hat{S}^{\mathrm{T}} T \hat{I}
$$

where $T$ is a $3 \times 3$ second-rank tensor with tensor elements 29

$$
T_{i j}=\frac{\mu_{0}}{4 \pi} g_{\mathrm{e}} \mu_{\mathrm{B}} g_{\mathrm{n}} \mu_{\mathrm{N}}\left\langle\psi_{0}\left|\frac{3 r_{i} r_{j}}{\mathrm{r}^{5}}-\frac{\delta_{i j}}{\mathrm{r}^{3}}\right| \psi_{0}\right\rangle
$$

where $\delta_{i j}$ is the Kronecker symbol $\left(\delta_{i j}=0\right.$ for $i \neq j$ and $\delta_{i j}=1$ for $i=j$ ).

The second term contained in the hyperfine tensor is derived from the above case when r approaches zero ${ }^{34]}$. The expression was first derived by Fermi ${ }^{35]}$ and is referred to as Fermi contact interaction. It scales with the probability of the electron spin density at the nucleus according to

$$
H_{\mathrm{F}}=a_{\mathrm{iso}} \hat{S}^{\mathrm{T}} \hat{I}
$$

with the the isotropic hyperfine coupling (hfc) constant

$$
a_{\text {iso }}=\frac{2 \mu_{0} \hbar}{3} g_{\mathrm{e}} \mu_{\mathrm{B}} g_{\mathrm{n}} \mu_{\mathrm{N}}\left|\psi_{0}(\mathrm{r}=0)\right|^{2}
$$

where $\mu_{0}$ is the permeability of the vacuum and $\left|\psi_{0}(\mathrm{r}=0)\right|^{2}$ the ground state electron spin density at the nucleus. Spin density at the nucleus either occurs directly due to s orbital contribution or is induced by the spin polarization mechanism from electron density in $\mathrm{p}, \mathrm{d}$, or f orbitals $\underline{299}$.

Thus, for hyperfine couplings in organic radical like the systems studied in this thesis, the Hamiltonian can be formulated as

$$
H_{\mathrm{HFC}}=\hat{S}^{\mathrm{T}} A \hat{I} \text { with } A=a_{\mathrm{iso}} \mathbb{1}+T
$$

with the identity matrix $\mathbb{1}$. 


\subsubsection{Nuclear quadrupole interaction}

The last term in the spin Hamiltonian given in Eq. 2.1 accounts for nuclei with nuclear spin quantum number $\mathrm{I}>1 / 2$. Physical origin of this term is the interaction of the nucleus' electric quadrupole moment with the electric field gradient. This field gradient arises from non-spherical charge distribution of electric charges around it. The Hamiltonian in energy units is given by

$$
H_{\mathrm{Q}}=\hbar \hat{I}^{\mathrm{T}} P \hat{I}
$$

where $P$ is the traceless nuclear quadrupole tensor. The general Hamiltonian in its principle axis frame can be rewritten as 28.

$$
H_{\mathrm{Q}}=\frac{e^{2} q P \hbar}{4 I(2 I+1)}\left[3 I_{z}^{2}-\mathrm{I}(\mathrm{I}-1) \mathbb{1}+\eta\left(I_{x}^{2}-I_{y}^{2}\right)\right]
$$

where $e q$ is the $z z$-component of the field gradient at the nucleus and $\eta=\frac{V_{x}-V_{y}}{V_{z}}$ the asymmetry parameter of the charge distribution with $\left|V_{z}\right| \geq\left|V_{y}\right| \geq\left|V_{z}\right|, V_{z}=e q$ and $0 \leq \eta \leq 1$, being zero for axial symmetry 30 . It is common to characterize the quadrupole interaction by quantifying the effective quadrupole coupling 32

$$
Q=\frac{e^{2} q P}{4 I(2 I+1)}(\text { and } \eta)
$$

typically given in frequency units. The corresponding diagonal elements of the tensor become $Q_{x}=Q(-1+\eta), Q_{y}=Q(-1-\eta)$ and $Q_{z}=2 Q$ and three Euler angles $(\alpha, \beta, \gamma)$ define the orientation of the principle axis system in the molecular axis or $g$-tensor reference system. Usually, the $g$-, $A$ - and $Q$-tensor principle frames do not coincide with each other.

\subsection{Time evolution of spin systems}

Spin dynamics comprises theoretical tools to describe the propagation of the paramagnetic spin system for instance between, during and after mw and/or rf pulses. A classical macroscopic description of spin dynamics considers the total magnetization as a vector in motion, known as the Bloch equations 36 . This description, however, does not represent interacting, coupled spins in a microscopic consideration. Such a system in motion is rather expressed by a density operator based on a quantum mechanical 
frame work ${ }^{[37]}$. The following section introduces the tools and transformation rules used for the description of the experiments in this thesis, for more detailed information the reader is referred to corresponding textboosk $28,29,32,38,39$.

\subsubsection{Density operator formalism}

The density operator is an alternative representation of the state of a quantum system for which a single wavefunction $|\Psi\rangle$ cannot satisfy all the information about the system. Thus, the density operator formalism is favorable to describe the time evolution of an ensemble of spins in a mixed state. A mixed state ensemble of $\left|\Psi_{k}(t)\right\rangle$ states is expressed in the density matrix by

$$
\rho(t)=\left|\Psi_{k}(t)\right\rangle\left\langle\Psi_{k}(t)\right|
$$

where $\left|\Psi_{k}(t)\right\rangle\left\langle\Psi_{k}(t)\right|$ is an outer product written in bra-ket notation. Further, the expectation value of an observable $A$ at the time $t$ in a state of $\rho$ is given by the Trace

$$
\langle A(t)\rangle=\operatorname{Tr}(\rho(t) A)
$$

The matrix elements of $\rho$ can be rationalized as follows. For a particular state $|i\rangle$ of several spins, the state of a single spin is described by

$$
|\Psi(t)\rangle=\sum_{i} c_{i}(t)|i\rangle
$$

where $c_{i}$ is the complex, time-dependent coefficient for the occupation of state $|i\rangle$. Consequently, the density matrix elements are

$$
\begin{aligned}
\rho(t)_{i j} & =\langle i|\rho(t)| j\rangle \\
& =\sum_{i, j}\left\langle i \mid \Psi_{k}(t)\right\rangle\left\langle\Psi_{k}(t) \mid j\right\rangle \\
& =\sum_{i, j} \overline{c_{i}(t) c_{j}(t)^{*}}|i\rangle\langle j|
\end{aligned}
$$

where the bar indicates the ensemble average over all spins and $c_{j}(t)^{*}$ is the complex conjugate of $c_{j}(t)$. The diagonal elements of this matrix $(i=j)$ give the probability of occupying a state $|i\rangle$. The off-diagonal elements indicate superposition of states, referred to as coherences. 
With this definition of the density matrix, the system dynamics under the effect of rf and mw pulses or during free evolution can be obtained from the Liouville-von Neumann equation

$$
\frac{\partial \rho(t)}{\partial t}=-\mathrm{i}[H(t), \rho(t)]
$$

The numerical treatment of this thesis considers pulses with constant amplitudes in the rotating frame, which results in time independent Hamiltonians. In this case, the solution of Eq. 2.20 becomes

$$
\rho\left(t_{i}\right)=\exp \left(-\mathrm{i} H t_{i}\right) \rho(0) \exp \left(\mathrm{i} H t_{i}\right)
$$

and further we call

$$
\begin{aligned}
U\left(t_{i}\right) & =\exp \left(-\mathrm{i} H t_{i}\right) \\
U^{-1}\left(t_{i}\right) & =\exp \left(\mathrm{i} H t_{i}\right)
\end{aligned}
$$

where $U\left(t_{i}\right)$ is the propagator during a time integral $\left[t_{i}\right]$ (e.g. $U\left(t_{\mathrm{CP}}\right)$ for the propagator of the CP pulse of $t_{\mathrm{CP}}=200 \mu$ s duration).

All experiments in this thesis consist of several pulses with for instance different amplitude, length or phase. Thus, the time evolution is expressed as product of propagators $U_{m}\left(t_{i}\right)$, each step characterized by a different Hamiltonian $H_{m}$

$$
\rho\left(m t_{i}\right)=U_{m}\left(t_{i}\right) \ldots U_{1}\left(t_{1}\right) \rho(0) U_{1}^{-1}\left(t_{1}\right) \ldots U_{m}^{-1}\left(t_{i}\right) .
$$




\subsection{Detection of hyperfine interactions via pulsed ENDOR spectroscopy}

Electron-nuclear double resonance (ENDOR) spectroscopy is one of the most frequently used techniques to direcly determine hyperfine couplings. There are two general approaches for ENDOR spectroscopy: either microwave is irradiated continuously (CW-ENDOR) or in pulsed mode (pulse ENDOR). Their general concept, however, remains identical: detection of electron-spin polarization losses induced by driving nuclear transitions as a function of the rf frequency. In CW-ENDOR, the monitored electron spin polarization is generated by saturating an EPR transition, thus limiting the application to spin systems/samples that can be partially saturated (for example radicals in solution). In contrast, pulsed ENDOR techniques rely on polarization transfer between electron spin and nucleus realized by a series of short pulses. In the following sections, the concept of ENDOR spectroscopy as well as the well-established pulse ENDOR techniques - Davies ENDOR ${ }^{5}$ and Mims ENDOR ${ }^{4]}$ - are summarized for the example of an electron spin $S=1 / 2$ coupled to a nuclear spin $I=1 / 2$.

\subsubsection{Analytically derived nuclear transition frequencies}

The general spin Hamiltonian of an electron spin $S=1 / 2$ coupled to one nulcear spin $I=1 / 2$ given in Eq. 2.1 is reformulated according to the above sections in the laboratory frame in energy units to

$$
\begin{aligned}
& H_{0}^{\mathrm{lab}}=H_{\mathrm{EZ}}+H_{\mathrm{NZ}}+H_{\mathrm{HFC}} \\
& H_{0}^{\mathrm{lab}}=\mu_{\mathrm{B}} \vec{B}_{0}^{\mathrm{T}} g \hat{S}-\mu_{\mathrm{N}} g_{\mathrm{n}} \vec{B}_{0}^{\mathrm{T}} \hat{I}+\hbar \hat{S}^{\mathrm{T}} A \hat{I} .
\end{aligned}
$$

Considering the high field approximation (HFA) (valid for instance for proton nuclei and deuterium nuclei at $94 \mathrm{GHz} / 3.4 \mathrm{~T}$ or higher) where the quantization axis of the electron and nuclear spins is aligned with the $z$-axis of $\overrightarrow{B_{0}}\left(\overrightarrow{B_{0}}=\left(0,0, B_{0}\right)\right), S_{x, y}$ contributions to the hyperfine interaction can be neglected $[\overline{29}$. Further, the simplest case of an isotropic $g_{\mathrm{e}}$ and positive $g_{\mathrm{n}}$ ( such as for ${ }^{1} \mathrm{H}$ and ${ }^{2} \mathrm{H}$ ) is assumed which together with the HFA simplify the Hamiltonian to 29

$$
H_{0}^{l a b} \approx \mu_{\mathrm{B}} B_{0} g_{\mathrm{e}} \hat{S}_{z}-\mu_{\mathrm{N}} g_{\mathrm{n}} B_{0} \hat{I}+\hbar S_{z} A I_{z}+\hbar S_{z} B_{y} I_{y}+\hbar S_{z} B_{x} I_{x}
$$


where $A=A_{z z}, B_{y}=A_{z y}$ and $B_{x}=A_{z x}$ are called secular and pseudo-secular hyperfine couplings.

After matrix expansion, the matrix form of this Hamiltonian is obtained to $\left.{ }^{34}\right]$

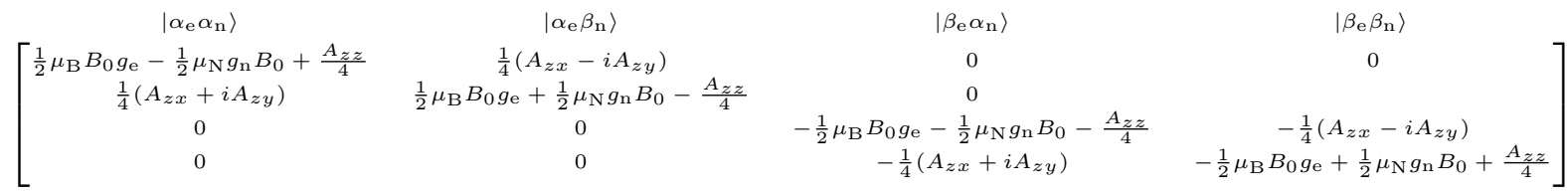

with the numbering of energy levels defined as $|1\rangle=\left|\alpha_{\mathrm{e}} \alpha_{\mathrm{n}}\right\rangle,|2\rangle=\left|\alpha_{\mathrm{e}} \beta_{\mathrm{n}}\right\rangle,|3\rangle=\left|\beta_{\mathrm{e}} \alpha_{\mathrm{n}}\right\rangle$, $|4\rangle=\left|\beta_{\mathrm{e}} \beta_{\mathrm{n}}\right\rangle$. Diagonalization of this Hamiltonian results in the energies 34

$$
\begin{aligned}
& E_{1,2}=\frac{\mu_{\mathrm{B}} B_{0} g_{\mathrm{e}}}{2} \pm \sqrt{\frac{1}{4}\left(A_{z x}^{2}-i A_{z y}^{2}\right)+\left(-\mu_{\mathrm{N}} g_{\mathrm{n}} B_{0}+\frac{1}{2} A_{z z}\right)} \\
& E_{3,4}=-\frac{\mu_{\mathrm{B}} B_{0} g_{\mathrm{e}}}{2} \pm \sqrt{\frac{1}{4}\left(A_{z x}^{2}-i A_{z y}^{2}\right)+\left(-\mu_{\mathrm{N}} g_{\mathrm{n}} B_{0}-\frac{1}{2} A_{z z}\right)} .
\end{aligned}
$$

Consequently, the nuclear transitions or ENDOR transitions (NMR [1-2] and NMR [3-4]) arise from the selection rules $\Delta m_{I}= \pm 1, \Delta m_{S}=0$ to

$$
\begin{aligned}
& E_{1}-E_{2}=\Delta E_{12}=\sqrt{\frac{1}{4}\left(A_{z x}^{2}-i A_{z y}^{2}\right)+\left(-\mu_{\mathrm{N}} g_{\mathrm{n}} B_{0}+\frac{1}{2} A_{z z}\right)} \\
& E_{3}-E_{4}=\Delta E_{34}=\sqrt{\frac{1}{4}\left(A_{z x}^{2}-i A_{z y}^{2}\right)+\left(-\mu_{\mathrm{N}} g_{\mathrm{n}} B_{0}-\frac{1}{2} A_{z z}\right)} .
\end{aligned}
$$

Considering additionally the nuclear Zeeman interaction to be larger than the hyperfine interaction, the $I_{x, y}$ contributions to the hyperfine interaction can be neglected as well and the equations reduce to 40

$$
\Delta E_{\mathrm{ENDOR}, I=1 / 2}=\left|\mu_{\mathrm{N}} g_{\mathrm{n}} B_{0} \pm \frac{1}{2} A_{z z}\right|
$$

which translates into two peaks observed in the ENDOR spectrum of one electron spin $S=1 / 2$ coupled to one nuclear spin $I=1 / 2$.

The ENDOR transitions for an electron spin $S=1 / 2$ coupled to a deuterium $(I=1)$ are derived analogously and result under HFA approximation for both spins to 40 .

$$
\Delta E_{\mathrm{ENDOR}, I=1}=\left|\mu_{\mathrm{N}} g_{\mathrm{n}} B_{0} \pm \frac{1}{2} A_{z z} \pm \frac{3}{2} Q_{z z}\right|
$$

Thus, in total four transitions split by the hyperfine and quadrupole coupling are 
predicted in the ENDOR spectrum of a $S=1 / 2, I=1$ spin system (see Fig. 2.2, C, center)).

A) Energy diagram $S=1 / 2, I=1$

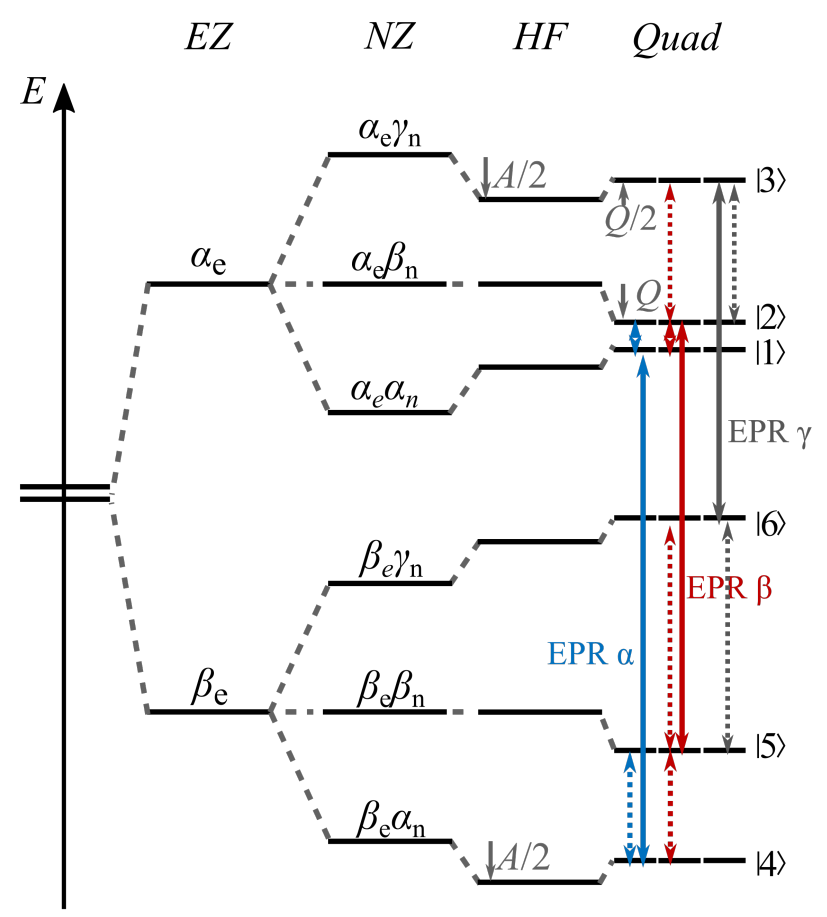

$\uparrow \uparrow \uparrow \begin{gathered}\text { EPR } \\ \text { transitions }\end{gathered}$

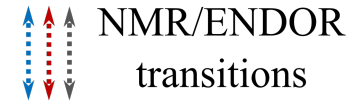

B) $E P R$ resonances for $A>0$

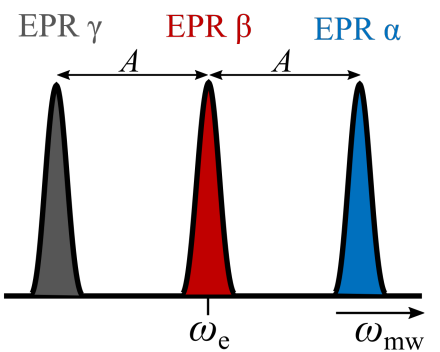

C) ENDOR resonances for $A>0$

ENDOR@EPR r

$[2-3]$

$[5-6]$

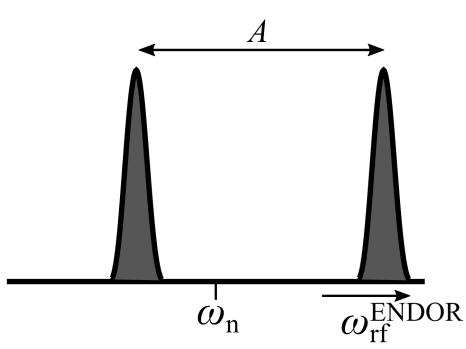

ENDOR@EPR $\beta$

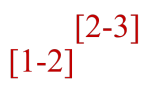

$[4-5]$

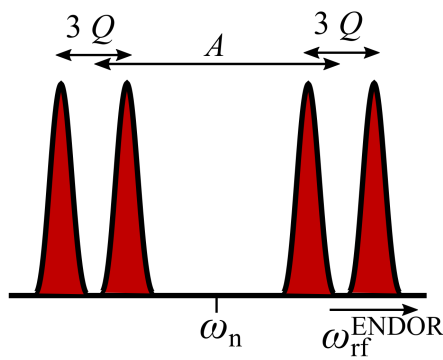

ENDOR@EPR $\alpha$

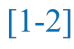

$[4-5]$

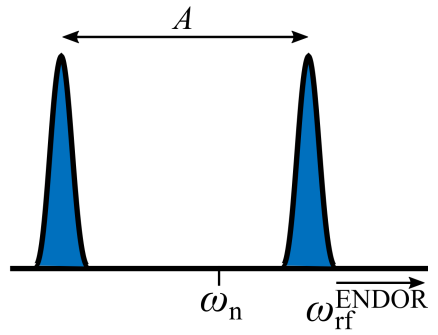

Figure 2.2: EPR and NMR transitions illustrated in the first-order energy diagram. A) First-order energy diagram for $S=1 / 2, I=1, A / 2 \pi>0, A / 2 \pi$ $<\omega_{\mathrm{n}}$ and $Q / 2 \pi>0$. We define $\beta_{\mathrm{e}}=-1 / 2, \alpha_{\mathrm{e}}=+1 / 2, \alpha_{\mathrm{n}}=+1, \beta_{\mathrm{n}}=0$ and $\gamma_{\mathrm{n}}$ $=-1$. In total three EPR transitions $\alpha, \beta, \gamma$ (straight arrows) and four different NMR transitions (dotted arrows) are assigned. $B$ ) Peak assignment in frequency domain EPR spectrum. C) Peak assignment in ENDOR spectrum. The number of the observed ENDOR lines relates to the number of allowed NMR transitions $\left(\Delta m_{I}= \pm 1\right)$ sharing an energy level with the respectively excited EPR transition.

However, for EPR selective ENDOR experiments, only two lines are predicted for 
selective excitation of EPR [1-4] (EPR $\alpha)$ and EPR [3-6] (EPR $\gamma)$, while four are obtained on EPR [2-5] (EPR $\beta)$ as illustrated in Fig. 2.2. Here, the EPR $\alpha$ transition is an electronic spin transition preserving the nuclear spin state $\alpha_{\mathrm{n}}, m_{I}=1\left(\left|\beta_{\mathrm{e}} \alpha_{\mathrm{n}}\right\rangle \rightarrow\left|\alpha_{\mathrm{e}} \alpha_{\mathrm{n}}\right\rangle\right)$, $\operatorname{EPR} \beta$ transition is an electronic spin transition preserving the nuclear spin state $\beta_{\mathrm{n}}$, $m_{I}=0\left(\left|\beta_{\mathrm{e}} \beta_{\mathrm{n}}\right\rangle \rightarrow\left|\alpha_{\mathrm{e}} \beta_{\mathrm{n}}\right\rangle\right)$ and EPR $\gamma$ is an electronic spin transition preserving the nuclear spin state $\gamma_{\mathrm{n}}, m_{I}=-1\left(\left|\beta_{\mathrm{e}} \gamma_{\mathrm{n}}\right\rangle \rightarrow\left|\alpha_{\mathrm{e}} \gamma_{\mathrm{n}}\right\rangle\right)$. The EPR and NMR/ENDOR transitions are illustrated in the energy diagram displayed in Fig. 2.2, A together with the resulting schematic electron-spin echo (ESE) detected EPR spectrum (Fig. 2.2, B) and EPR selective ENDOR spectra (Fig. 2.2, C). The observation of only two ENDOR transitions on EPR $\alpha$ and EPR $\gamma$ is due the fact that only two allowed NMR transitions $\left(\Delta m_{I}= \pm 1\right)$ can be driven on basis of the excited EPR transitions. For instance, an ENDOR experiment with selective excitation on EPR $\alpha$ does not report on the NMR transition [5-6] because a manipulation of these energy levels will not have an effect on the detected transition EPR [1-4].

\subsubsection{Davies ENDOR}

It was 45 years ago, in 1974, when E. R. DAviES published "a new pulsed ENDOR technique" referred two as "method two", which is nowadays a well-established ENDOR sequences known as Davies ENDOR $[5$. In Fig. 2.3, the Davies ENDOR pulse sequence and the polarization transfer scheme is illustrated schematically for an $S=1 / 2, I=1 / 2$, $A>0$ spin system 40$]$. The sequence can be divided into three parts: a preparation part which generates electron-nuclear polarization, an ENDOR part where an NMR transition is driven and a read-out step of the ENDOR information. The first pulse of the sequence is a $\pi$ inversion pulse which selectively (soft pulse) inverts the population of an EPR transition. Following the notation in 40$]$, this preparation pulse creates the electron-nuclear two-spin order $2 S_{z} I_{z}$ according to

$$
\rho_{0}=-S_{z}=S_{z}\left(-I^{\alpha}-I^{\beta}\right) \stackrel{\pi S_{x} I^{\alpha}}{\longrightarrow} S_{z}\left(I^{\alpha}-I^{\beta}\right)=2 S_{z} I_{z}=\rho_{\mathrm{prep}}
$$

with a selective $\pi$-pulse of $x$-phase applied on the EPR [1-3] transition of the density matrix $\rho_{0}$ at the beginning of the sequence defined as $\rho_{0}=-S_{z}$. The electron-nuclear two-spin order is defined as polarization of both the two ESR and the two NMR transitions, however no net polarization. 


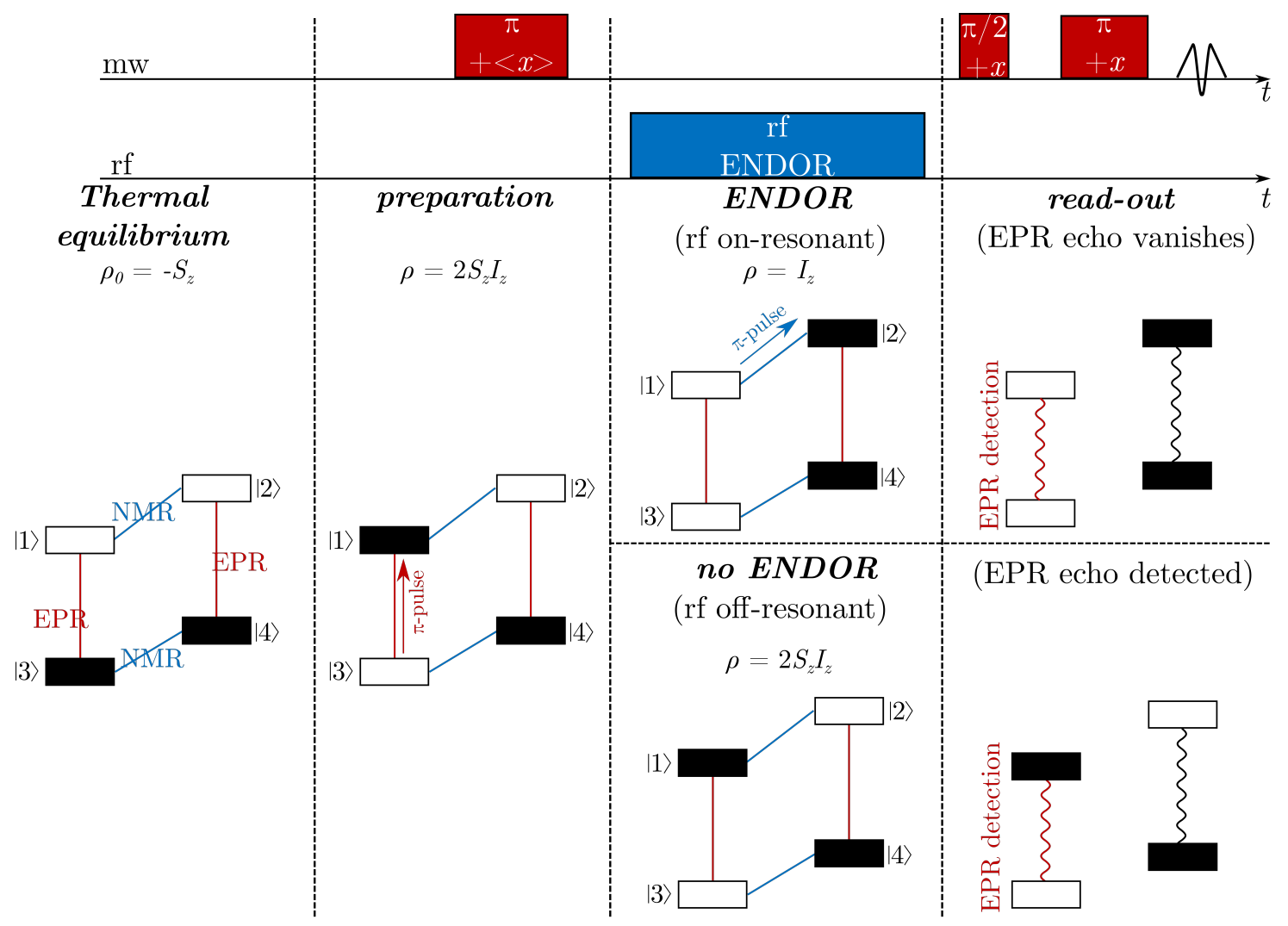

Figure 2.3: Davies ENDOR pulse sequence and population transfer scheme. Full boxes represent high population, empty boxes represent low population of the corresponding energy level. At the preparation stage, a selective mw pulse on EPR [1-3] creates the two-spin order $2 S_{z} I_{z}$. Afterwards, a frequency swept rf pulse selectively inverts on-resonant with e.g. NMR [1-2] the populations (ENDOR step). If the rf pulse is off-resonant with an NMR transition, the prepared population pattern remains. The resulting longitudinal electron spin polarization is transferred to electron spin coherence and detected via a Hahn-echo sequence. The ENDOR effect is observed as the difference in electron spin polarization between the rf ENDOR pulse off-resonant and on-resonant with an NMR transition. Figure adapted from [40].

In the notation following the product operator formalism as described in 41 the nuclear spin polarization operators that select the EPR line associated with the $\alpha$ and $\beta$ nuclear spin states are denoted by $I^{\alpha}$ and $I^{\beta}$. Likewise, $S^{\alpha}$ and $S^{\beta}$ denote nuclear spin polarization operators that select the NMR line associated with the $\alpha$ and $\beta$ electron spin states ${ }^{[40]}$. Further, in the introduced spin system $S_{x} I^{\alpha}$ and $S_{x} I^{\beta}$ refer to the allowed EPR transitions between states $\left|\alpha_{\mathrm{e}} \alpha_{\mathrm{n}}\right\rangle \rightarrow\left|\beta_{\mathrm{e}} \alpha_{\mathrm{n}}\right\rangle([1-3])$ and $\left|\alpha_{\mathrm{e}} \beta_{\mathrm{n}}\right\rangle \rightarrow\left|\beta_{\mathrm{e}} \beta_{\mathrm{n}}\right\rangle([2-4])$, respectively. Correspondingly, the operators $I_{x} S^{\alpha}$ and $I_{x} S^{\beta}$ refer to the allowed NMR transitions between states $\left|\alpha_{\mathrm{e}} \alpha_{\mathrm{n}}\right\rangle \rightarrow\left|\alpha_{\mathrm{e}} \beta_{\mathrm{n}}\right\rangle([1-2])$ and $\left|\beta_{\mathrm{e}} \alpha_{\mathrm{n}}\right\rangle \rightarrow\left|\beta_{\mathrm{e}} \beta_{\mathrm{n}}\right\rangle([3-4])$.

In the next step of the sequence, a frequency swept rf pulse selectively inverts on 
resonance with e.g. NMR transition [1-2] the populations according to

$$
2 S_{z} I_{z} \stackrel{\beta_{2} I_{x} S^{\alpha}}{\longrightarrow} \frac{1}{2}\left(1+\cos \beta_{2}\right) S_{z}\left(I^{\alpha}-I^{\beta}\right)-\frac{1}{2}\left(1-\cos \beta_{2}\right) I_{z}-\sin \beta_{2} I_{y} S^{\alpha}=\rho_{\mathrm{ENDOR}},
$$

obtained after a rf pulse along the $x$-axis with flip angle $\beta_{2}$. Finally, the two-pulse Hahnecho detection sequence $\pi / 2-\tau-\pi-\tau-$ echo is used to read-out the $z$-magnetization of the initially driven EPR transition according to

$$
\rho_{\mathrm{ENDOR}} \stackrel{\pi / 2 S_{x} I^{\alpha}}{\longrightarrow} \stackrel{\tau}{\rightarrow} \stackrel{\pi S_{x} I^{\alpha}}{\longrightarrow} \stackrel{\tau}{\rightarrow} \frac{1}{2}\left(1+\cos \beta_{2}\right) S_{y} I^{\beta}=\rho_{\mathrm{echo}}
$$

Although noted as selective (soft) pulses, the detection pulses are usually applied as non-selective (hard) pulses in order to improve the overall detected echo intensity since in general very few spins are excited. Accordingly, the detection does not require selective pulses, however, the preparation mandatorily does in order to generate the electronnuclear two-spin order. A non-selective preparation pulse will create $S_{z}$, inappropriate for the following rf ENDOR polarization transfer. At the time point of the echo, the ENDOR spectrum is mapped via the $S_{y}$ operator associated with transverse magnetization to 40

$$
I_{\text {echo }}\left(\beta_{2}\right)=\left\langle S_{y}\left(t_{\text {echo }}\right)\right\rangle=\operatorname{Tr}\left(\rho_{\text {echo }} S_{y}\right)=-\frac{1}{4}\left(1+\cos \beta_{2}\right) .
$$

The ENDOR effect, however, is defined as change in electron spin polarization (longitudinal) due to the rf pulse. Accordingly, $\left\langle S_{y}\left(t_{\text {echo }}\right)\right\rangle$ at the time point of the echo reports on the ENDOR effect on the excited EPR transition. It is clarified, that the phase of the mw pulses defines the detection operator such that for mw pulses with $x$-phase $\left\langle S_{y}\left(t_{\text {echo }}\right)\right\rangle$ is monitored and for mw pulses with $y$-phase $\left\langle S_{x}\left(t_{\text {echo }}\right)\right\rangle$. The ENDOR effect can be rationalized by the turning angle $\beta_{2}$ referring to either $\beta_{2}=0$ (rf frequency does not drive an NMR transitions, EPR echo detected) or $\beta_{2}=\pi$ (rf frequency drives an NMR transition, EPR echo vanishes). The two cases result in echo intensities as follows

$$
\begin{aligned}
& I_{\text {echo }}\left(\beta_{2}=0\right)=\left|-\frac{1}{4}(1+\cos 0)\right|=\frac{1}{2}, \\
& I_{\text {echo }}\left(\beta_{2}=\pi\right)=\left|-\frac{1}{4}(1+\cos \pi)\right|=0,
\end{aligned}
$$

This can be translated into a so-called ENDOR efficiency parameter which represents 
the relative echo change upon rf excitation by

$$
F_{\text {ENDOR }}=\frac{1}{2}\left|\frac{I_{\text {echo }}\left(\mathrm{rf}_{\text {off }}\right)-I_{\text {echo }}\left(\mathrm{rf}_{\text {on }}\right)}{I_{\text {echo }}\left(\mathrm{rf}_{\text {off }}\right)}\right| .
$$

The maximum Davies ENDOR efficiency $\left(\beta_{2}=\pi\right)$ refers to $F_{\text {ENDOR }}=0.5$ and a formally disappearing echo $\left(I_{\text {echo }}(\pi)=0\right)$.

The above description nicely reflects the Davies ENDOR intensities for ideal selective mw pulses acting on one spin packet. However, typical ENDOR experiments are performed on frozen solution samples, for which the observed inhomogenously broadened EPR line consists of a superposition of multiple spin packets with distinct Larmor frequencies 2 (see also Subsec. 2.3.4). The following phenomenological description of the Davies ENDOR sequence applied on a broad EPR line is illustrated in Fig. 2.4.

The Davies ENDOR $\pi$-preparation pulse excites a range of spin packets of the inhomogeneously broadened EPR line according to its excitation profile. This process is so-called hole burnt into the EPR spectrum. An approximation of the excitation profile for a short rectangular pulse in the frequency domain can be obtained from Fourier transformation of the pulse shape in the time domain 29. For a rectangular pulse extending from $t=-t_{\mathrm{p}} / 2$ to $t=+t_{\mathrm{p}} / 2$ this results in the absolute-value spectrum given by 29

$$
S\left(\Delta \omega_{\mathrm{e}}\right)=\left|\frac{\sin \left(\Delta \omega_{\mathrm{e}} t_{\mathrm{p}} / 2\right)}{\left(\Delta \omega_{\mathrm{e}} / 2\right)}\right|
$$

with $\Delta \omega_{e}$ is the resonance frequency offset and $t_{p}$ is the pulse length. The excitation bandwidth of such a pulse (in experimentally relevant frequency units) scales with

$$
\Delta \nu_{1 / 2} \approx \frac{1}{t_{\mathrm{p}}}
$$

thus, with the reciprocal of the pulse length. Obviously, only the spin packets excited by the pulse participate in the ENDOR experiment and can be individually described by the polarization scheme shown in Fig. 2.3. Describing the ENDOR sequence from the perspective of the non-excited spin packets, thus, from the remaining $z$-magnetization observed in the EPR spectrum, the frequency swept rf pulse on-resonant with one nuclear transition frequency during the ENDOR step becomes apparent by magnetization transfer from the outside to the center of the hole (Fig. 2.4. C and E). The created side holes are separated by the hfc $A$ from the central hole and indeed reduce the intensity

${ }^{2}$ There are various sources for inhomogeneity ranging from anisotropy of $g$ - and $A$-tensors to distributions of field intensity $B_{1}$. For further detailed information on EPR line broadening see 28, 29. 
of the central hole; its gets refilled. This refilled hole corresponds to the situation of vanishing echo intensity for the spin packets which participate in polarization transfer during the ENDOR sequence.

A)

$\pi$ - preparation
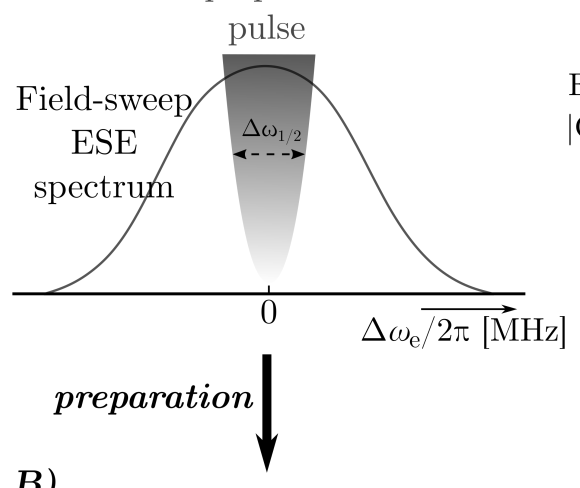

B) central hole

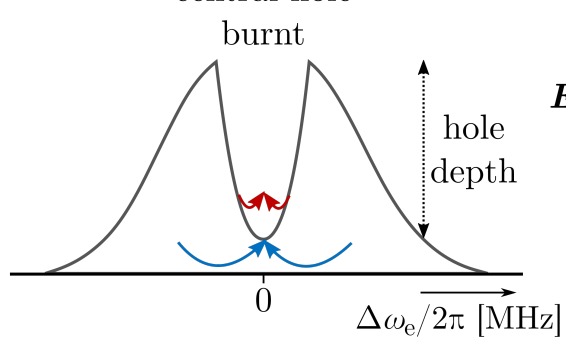

ENDOR

off-resonant

C)

detection

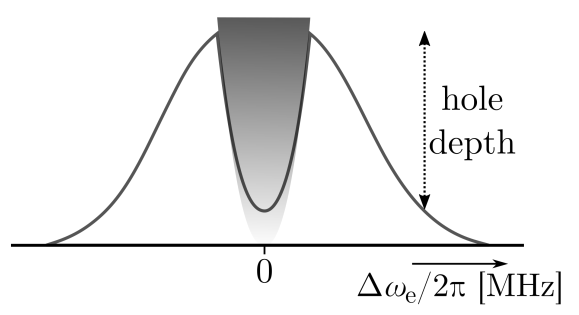

F)

Davies ENDOR blind spot

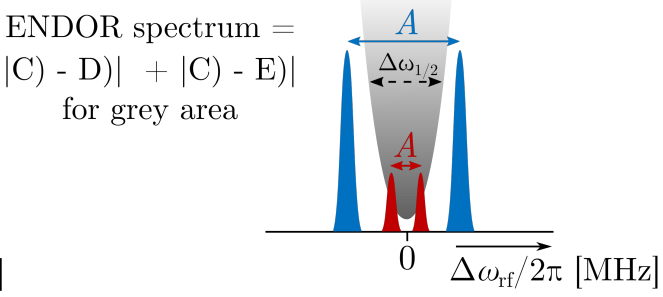

$\uparrow$ read-out

E) detection

ENDOR

$\stackrel{\text { small } A}{\longrightarrow}$

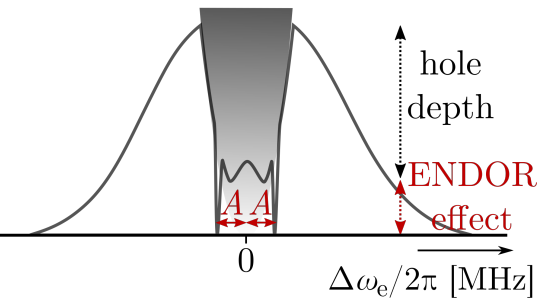

ENDOR

D)

D) detection

Figure 2.4: Illustration of hole burning in Davies ENDOR. $A) \rightarrow B$ ) The selective $\pi$-preparation pulse excites a fraction of the inhomogenously broadened EPR line, a so-called hole is burnt into the EPR line. $C$ ) The rf ENDOR pulse is off-resonant to an NMR transition, thus the initially burned hole remains unaltered. $D, E$ ) The rf ENDOR pulse on-resonant with an NMR transition creates side holes which are separated by the hfc $A$ to the central hole. This reduces the intensity of the central hole. $F)$ The ENDOR spectrum is observed from the difference between the on-resonant and off-resonant rf cases. The detected difference will be large for large $A$ (blue), however, decreases for small $A$ (red) with respect to the width oft the central hole. For $A$ smaller than the FWHM of the preparation pulse, central hole and side hole overlap, polarization transfer becomes incomplete and the ENDOR effect decreases.

Finally, the detection step monitors the central hole depth after the rf ENDOR pulse. If the rf ENDOR pulse was on-resonant with an NMR transition, the central hole has been 
refilled and the hole depth becomes zero. An off-resonant rf ENDOR pulse will leave the initial hole depth unaltered. The difference between the on-resonant and off-resonant rf case is referred to as ENDOR effect being proportional to the extent of refilling.

The described hole burning introduces a sensitivity limit for small hyperfine couplings. The polarization transfer during rf irradiation induces a detectable echo change only as long as $A$ exceeds the mw pulse bandwidth (Fig. 2.4, D). For $A$ smaller than the FWHM of the mw preparation pulse, central hole and side hole overlap, polarization transfer becomes incomplete and the desired change in echo intensity is diminished (Fig. 2.4 E). The consequence is a so-called blind spot in the center of the ENDOR spectrum (Fig. 2.4, F). Intrinsically, the applied selective $\pi$-preparation pulse length determines the FWHM of the excitation bandwidth of the pulse as well as the FWHM of the blind spot observed in the ENDOR spectrum. This correlation reflects the lower sensitivity limit for the detection of small hfcs in Davies ENDOR and makes the sequence favorable for the detection of large hfcs.

Since microwave technology improved over the last decades and pulse shaping has been introduced to EPR, the preparation inversion pulse in Davies ENDOR can be substituted by a more selective shaped pulse ${ }^{422}$. This results in an essentially narrower hole burned into the EPR spectrum, thus improving the sensitivity for detection of small hfcs. This approach, however, requires state-of-the-art instrumentation (arbitrary wave form generator) available at the spectrometer. Furthermore, it is reported ${ }^{42}$ to be particularly advantageous in cases where a good signal-to-noise of the echo transients can be observed, because the acquisition of the full echo transients as a function of rf ENDOR pulse frequency is required in this approach. However, for detection of small hfcs the method of choice is the Mims ENDOR sequence, described in the following subsection.

\subsubsection{Mims ENDOR}

Since published in 1965 by B. Mims 4 , the so-called Mims ENDOR sequence is the most commonly used ENDOR technique for detection of small hyperfine interactions. In a phenomenological description of the Mims ENDOR sequence, three $\pi / 2 \mathrm{mw}$ pulses create a stimulated echo at the time $\tau$ after the last mw pulse. However, an rf pulse on-resonant with an NMR transition between the second and the third mw pulse will shift the electron spin polarization such that it does not refocus anymore and the stimulated echo vanishes. Specifically, the required two-spin order $2 S_{z} I_{z}$ is generated by 
a preparation step consisting of two non-selective (hard) $\pi / 2$ mw pulses with phase $+x$, separated by a time $\tau$ as illustrated in Fig. 2.5.

The first pulse turns the $z$-magnetization into the transverse plane where it processes

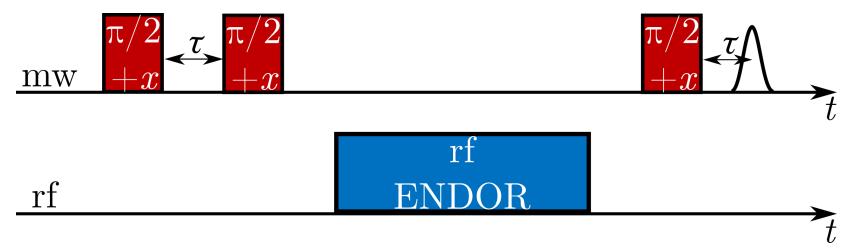

Figure 2.5: Pulse sequence for Mims ENDOR spectroscopy. Two nonselective $\pi / 2 \mathrm{mw}$ pulses separated by the free evolution time $\tau$ generate a $\tau$ dependent polarization grating in the longitudinal plane. In the ENDOR step, electron spin polarization gets shifted due to the rf pulse on-resonant with an NMR transition. Due to the shifting, the electron spin polarization does not refocus to an echo.

during the free evolution time $\tau$. The second mw pulse transfers the created polarization grating into the longitudinal plane ( $B_{0}$ direction) according to $\underline{40}$

$$
\begin{aligned}
\rho_{0}=-S_{z} \stackrel{\pi / 2 S_{x}}{\longrightarrow} S_{y} \stackrel{\tau}{\rightarrow} \stackrel{\pi / 2 S_{x}}{\longrightarrow} & S_{z} \cos \left(\frac{A}{2} \tau\right) \cos \left(\Delta \omega_{\mathrm{e}} \tau\right) \\
& -2 S_{z} I_{z} \sin \left(\frac{A}{2} \tau\right) \sin \left(\Delta \omega_{\mathrm{e}} \tau\right)=\rho_{\mathrm{prep}}
\end{aligned}
$$

where only the terms relevant for the echo detection are considered and non-detected coherences are omitted. Both terms are obtained as functions of the hfc $A$ as well as the electron spin offset $\Delta \omega_{\mathrm{e}}$. The ENDOR relevant two-spin order is created if $A \neq 0$ and $\Delta \omega_{\mathrm{e}} \neq 0$. This condition already reflects the origin of the high sensitivity which is the broad excitation bandwidth associated with hard, non-selective pulses compared to the soft preparation pulse in Davies ENDOR. Typical $\pi / 2$ pulse length for Mims ENDOR are $t_{\pi / 2} \approx 20-40 \mathrm{~ns}$ and for Davies ENDOR typical preparation pulses last $t_{\pi} \approx 200-400 \mathrm{~ns}$. Thus, there is a difference in excitation bandwidth between the two sequences by almost an order of magnitude according to the correlation given in Eq. 2.43. This preparation stage is followed by a frequency swept rf pulse which selectively inverts e.g. on-resonant with NMR transition [1-2] the populations according to

$$
\begin{aligned}
\rho_{\text {prep }} \stackrel{\beta_{2} I_{x} S^{\alpha}}{\longrightarrow} & S_{z} \cos \left(\frac{A}{2} \tau\right) \cos \left(\Delta \omega_{\mathrm{e}} \tau\right)-\left[2 S_{z} I_{z}\left[\frac{1}{2}\left(1+\cos \beta_{2}\right)\right]\right. \\
& +I_{z}\left[\frac{1}{2}\left(1-\cos \beta_{2}\right)\right]+I_{y}\left[\frac{1}{2}\left(\sin \beta_{2}\right)\right] \\
& \left.+2 S_{z} I_{y}\left[\frac{1}{2}\left(\sin \beta_{2}\right)\right]\right] \sin \left[\left(\frac{A}{2}\right) \tau\right] \sin \left(\Delta \omega_{\mathrm{e}} \tau\right)=\rho_{\text {ENDOR }}
\end{aligned}
$$


obtained after a rf pulse along the $x$-axis with flip angle $\beta_{2}$. A final non-selective mw $\pi / 2$ pulses with phase $+x$ creates a so-called stimulated echo at the time $2 \tau$ after the rf pulse:

$$
\begin{aligned}
\rho_{\text {ENDOR }} \stackrel{\pi / 2 S_{x}}{\longrightarrow} \underset{\tau}{\rightarrow} & -S_{y}\left[\cos ^{2}\left[\left(\frac{A}{2}\right) \tau\right] \cos ^{2}\left(\Delta \omega_{\mathrm{e}} \tau\right)\right. \\
& \left.+\frac{1}{2} \sin ^{2}\left[\left(\frac{A}{2}\right) \tau\right] \sin ^{2}\left(\Delta \omega_{\mathrm{e}} \tau\right)\left(1+\cos \beta_{2}\right)\right]=\rho_{\text {echo }} .
\end{aligned}
$$

The ENDOR effect can be rationalized by the turning angle $\beta_{2}$ referring to either $\beta_{2}=0$ (rf frequency does not drive an NMR transition, stimulated echo detected) or $\beta_{2}=\pi$ (rf frequency drives an NMR transition, stimulated echo vanishes). The corresponding intensities density matrices are

$$
\begin{aligned}
& \rho_{\text {echo }}\left(\beta_{2}=0\right)=-S_{y}\left[\cos ^{2}\left[\left(\frac{A}{2}\right) \tau\right] \cos ^{2}\left[\Delta \omega_{\mathrm{e}} \tau\right]+\sin ^{2}\left[\left(\frac{A}{2}\right) \tau\right] \sin ^{2}\left[\Delta \omega_{\mathrm{e}} \tau\right]\right] \\
& \rho_{\text {echo }}\left(\beta_{2}=\pi\right)=-S_{y}\left[\cos ^{2}\left[\left(\frac{A}{2}\right) \tau\right] \cos ^{2}\left[\Delta \omega_{\mathrm{e}} \tau\right]\right] .
\end{aligned}
$$

These terms describe the polarization transfer for one spin packet with a specific electron spin offset $\Delta \omega_{\mathrm{e}}$. The corresponding Mims ENDOR intensity associated with all excited EPR contributions is obtained from averaging over all resonance offsets for a turning angle $\beta_{2}=\pi$ :

$$
I_{\text {echo }}(\pi)=\frac{1}{4}[1+\cos (A \tau)]
$$

Accordingly, the ENDOR efficiency is obtained to ${ }^{43]}$

$$
F_{\mathrm{ENDOR}}=\frac{1}{4}[1-\cos (A \tau)]
$$

The maximum Mims ENDOR efficiency $\left(\beta_{2}=\pi\right)$ is obtained to $F_{\text {ENDOR }}=0.5$, equal to Davies ENDOR. Although the maximum ENDOR efficiency is equal for both sequences, their relative echo intensities are not directly comparable because of the already introduced different excitation bandwidths resulting in significantly different number of spin packets contributing to the echos 40 .

From Eq. 2.50 it becomes evident that $F_{\text {ENDOR }}$ reaches its maximum for $\tau=\frac{(2 n+1) \pi}{A}$ and its minimum for $\tau=\frac{2 n \pi}{A}, n=0,1,2$, etc.. The occurring minima in the spectrum at $A=\frac{n}{\tau}, n=0,1,2, \ldots$, are so-called Mims ENDOR blind spots and are described by 
the function

$$
I_{\text {blind spots }} \propto \frac{1}{4}[1+\cos (A \tau)]
$$

This blind spot issue is the major disadvantage of the Mims ENDOR sequence and usually leads to recording of several Mims ENDOR spectra with different $\tau$ values to ensure completeness of the observed hf-tensor features. This additional experimental need compromises the in principle high sensitivity of the sequence.

Finally, both Davies and Mims ENDOR are predicted with zero intensity at the Larmor frequency of the detected nucleus which is experimentally only rarely observed (for instance when detecting ${ }^{1} \mathrm{H}$ of a sample in a deuterated solvent). In most cases, a non-structured so-called matrix peak originating form polarization inversion by the rf pulse of a large number of nuclear spins weakly coupled to the paramagnetic species is observed at the center of the ENDOR spectrum.

\subsubsection{High-field ENDOR spectroscopy}

The advancing development of high-frequency microwave technology in the past years facilitated versatile applications of EPR and ENDOR spectroscopy at high fields/high frequencies 7, 17, 44, 46. The field dependent contributions of the Hamiltonians introduced in Section 2.1.1 are the electron and nuclear Zeeman interaction. With increasing magnetic fields for example from $9 \mathrm{GHz} / 0.3 \mathrm{~T}$ to $94 \mathrm{GHz} / 3.4 \mathrm{~T}$ or even $263 \mathrm{GHz} / 3.4 \mathrm{~T}$, the electron Zeeman interaction becomes the dominant contribution in the spin Hamiltonian for the a vast majority of organic radicals such as the tyrosyl radical studied in this thesis. In samples with molecules randomly oriented, like in powder samples or frozen solutions, each molecular orientation relative to the external magnetic field (connected to the $g$ - and $A / Q$-tensor principle axis frames as described in Sec. 2.1.1) refers to a different interaction strength of the electron (and nuclear) Zeeman term which translates into slightly different resonance conditions. At high fields/frequencies these distinct resonances can be separated and thus a so-called powder pattern spectrum is observed where the main $g$-tensor elements can be identified as illustrated in Fig. 2.6. However, almost all pulse experiments at these frequencies are operated with mw pulses which excite only a fraction of the EPR spectrum, for instance the preparation pulse in a Davies ENDOR experiment as discussed in Subsec. 2.3.2. Since the excited fraction refers to spin packets with a particular molecular orientation relative to the external 
magnetic field, the pulse is referred to as orientation selective (Fig. 2.6, B). This orientation selection results in "incomplete“-powder pattern ENDOR spectra and thus contains information on the mutual $g$-and $A / Q$-tensor orientation. This information can be further used to gain structural information on the nuclei in the close vicinity of the paramagnetic center 16, 17, 47.

A) X-Band

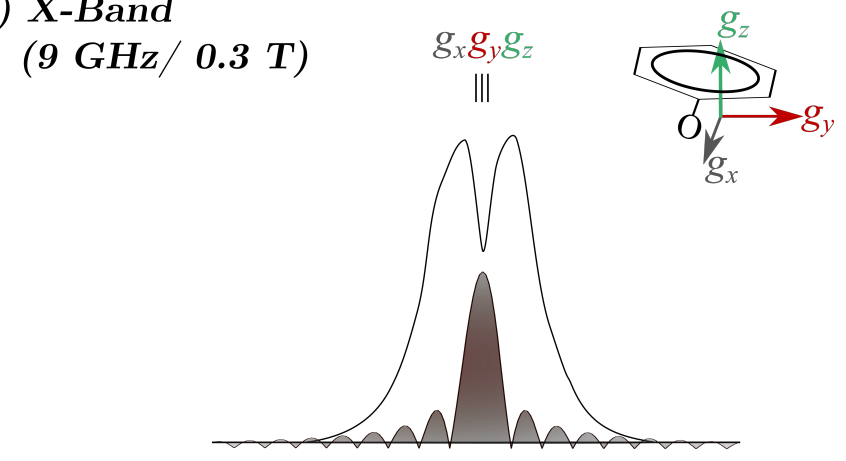

B) W-Band

(94 GHz, $3.4 \mathrm{~T})$
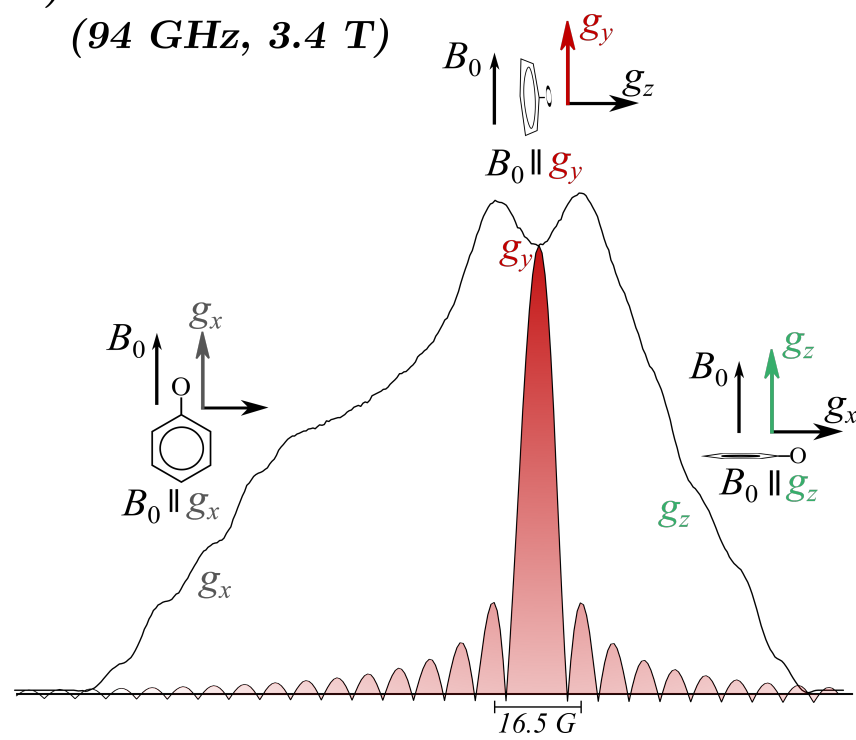

Figure 2.6: Representative ESE-detected field sweep spectra of a tyrosyl radical at $9 \mathbf{G H z}$ and $\mathbf{9 4} \mathbf{G H z}$. A) $9 \mathrm{GHz} / 0.3 \mathrm{~T}$ ESE-detected field sweep spectrum of a representative $\mathrm{Y}^{\bullet}$ and $g$-tensor principle axis orientations in the molecular frame. $g_{x}, g_{y}$ and $g_{z}$ are not resolved in the spectrum. Consequently, the shown $t_{\mathrm{mw}}=40 \mathrm{~ns}$ mw pulse excites a multitude of molecular orientations. B) At $94 \mathrm{GHz} / 3.4 \mathrm{~T}$, the $g$-tensor resolution is increased such that the $t_{\mathrm{mw}}=40 \mathrm{~ns} \mathrm{mw}$ pulse selects molecules with particular orientations relative to the external magnetic field (illustrated for excitation at $\left.B_{0} \| g_{y}\right)$.

Beside the enhanced $g$-tensor resolution in the high-field/high-frequency field sweep spectrum, the simultaneously increasing nuclear Zeeman interaction improves the separation of nuclear Larmor frequencies in ENDOR spectroscopy. For example the 
Larmor frequencies of the most frequently used target nuclei in ENDOR spectroscopy at $9 \mathrm{GHz} / 0.3 \mathrm{~T}$ are $\nu_{\mathrm{n}}^{14 \mathrm{~N}}=3.1 \mathrm{MHz}, \nu_{\mathrm{n}}^{15 \mathrm{~N}}=4.3 \mathrm{MHz}, \nu_{\mathrm{n}}^{17 \mathrm{O}}=5.7 \mathrm{MHz}, \nu_{\mathrm{n}}^{2 \mathrm{H}}=6.5 \mathrm{MHz}$, $\nu_{\mathrm{n}}^{1 \mathrm{H}}=42.6 \mathrm{MHz}$ and result in partial overlap of resonances from different nuclei assuming typical hfcs of 2-10 MHz. The situation improves at $3.4 \mathrm{~T} / 94 \mathrm{GHz}$ where the Larmor frequences are observed mostly separate at frequencies of $\nu_{\mathrm{n}}^{14 \mathrm{~N}}=10.5 \mathrm{MHz}$, $\nu_{\mathrm{n}}^{15 \mathrm{~N}}=14.7 \mathrm{MHz}, \nu_{\mathrm{n}}^{17 \mathrm{O}}=19.6 \mathrm{MHz}, \nu_{\mathrm{n}}^{2 \mathrm{H}}=22.2 \mathrm{MHz}, \nu_{\mathrm{n}}^{1 \mathrm{H}}=144.7 \mathrm{MHz}$.

Finally, the relative sensitivity improves with higher fields/frequencies due to the increasing electron spin polarization. The polarization $P$ is a measure of the difference in population between two energy levels and is determined by the Boltzmann factor

$$
P=\frac{p_{1}-p_{2}}{p_{1}+p_{2}} \sim \tanh \frac{\Delta E}{2 k_{\mathrm{B}} T}
$$

for a $S=1 / 2$ spin system. Thus, for $\Delta E \ll 2 k_{\mathrm{B}} T$ the polarization is approx. proportional to the energy difference $\Delta E$ between two spin levels which itself is a function of the applied magnetic field $B_{0}$ and the gyromagnetic ratio $\gamma_{\mathrm{e}, \mathrm{n}}=\left(\mu_{\mathrm{B}, \mathrm{N}} \cdot g_{\mathrm{e}, \mathrm{n}}\right) / \hbar$ as following from Eq. 2.3. The latter is a measure for the ratio of the magnetic moment of a spin-carrying particle (electron or nucleus) to its angular momentum and accounts for the fundamentally different interaction energies and timescales between electron and nuclear spins. For instance, the gyromagnetic ratio of the electron is $\approx 660$ times larger than for the proton, thus their energy splitting and consequently their polarization differs by almost three orders of magnitude. The described field dependency of $P$ results for electron spins at $T=10 \mathrm{~K}$ to $P(0.3 \mathrm{~T}) \approx 2.3 \%$ and $P(3.4 \mathrm{~T}) \approx 22 \%$, thus an order of magnitude higher polarization is achieved at $94 \mathrm{GHz} / 3.4 \mathrm{~T}$ compared to $9 \mathrm{GHz} / 0.3 \mathrm{~T}$ [48]. The increased polarization amounts for high sensitivity detection of small amount of free radicals. An advantage for protein studies with limited available sample amount.

\subsubsection{Nuclear saturation in ENDOR spectroscopy}

Beside the ENDOR sequence-specific disadvantages, the ENDOR performance in general can suffer from saturation effects as reported in several publications $26,49,51]$. Because ENDOR experiments are almost exclusively performed with long term averaging of the signal $(>12 \mathrm{~h})$, the repetition rate of the sequence becomes crucial. Following the formalism as presented in 49$]$ the population pattern of a spin system at the end of the Davies ENDOR sequence is non-Boltzmann equilibrium and is committed to fast electron spin relaxation, slow nuclear relaxation as well as zero- and double-quantum relaxation pathways as shown in Fig. 2.7. 


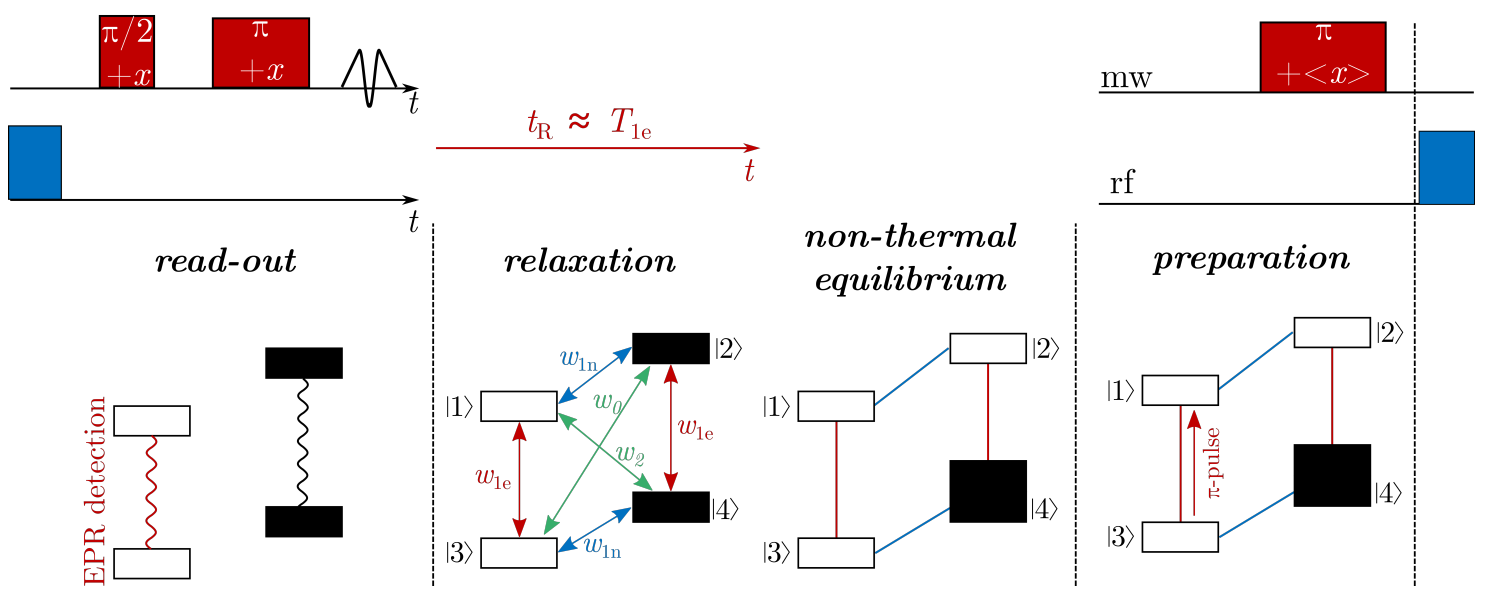

Figure 2.7: Polarization pattern between two Davies ENDOR sequences repeated for signal averaging. At the end of one shot (compare with Fig. 2.3) electron spin polarization vanishes and nuclear polarization is created. In general, full boxes represent high population, empty boxes represent low population of the corresponding energy level. Before the subsequent shot the system undergoes relaxation during the shot repetition time $t_{\mathrm{R}}$. Possible relaxation pathways and corresponding relaxation rates $\left(w_{i}\right)$ are via electron spin relaxation $\left(T_{1 \mathrm{e}}, w_{1 \mathrm{e}}\right.$, red arrows), nuclear spin relaxation ( $T_{1 \mathrm{n}} w_{1 \mathrm{n}}$, blue arrows) as well as zero quantum and double quantum relaxation $\left(w_{0}, w_{2}\right.$, green arrows). If $T_{1 \mathrm{e}}>T_{1 \mathrm{n}}$ and following the experimental protocol with a sequence repetition rate on the order of the electron spin relaxation $t_{\mathrm{R}} \sim T_{1 \mathrm{e}}$, the repetition rate is not sufficient to re-establish thermal equilibrium before the subsequent shot. Thus, this following scan will be less efficient since the required electron spin polarization for the preparation pulse is not restored. Adapted from 52 .

Since thermal equilibrium cannot be re-established from fast relaxation pathways only $\left(w_{1 \mathrm{e}}\right.$ in Fig. 2.7), the slow nuclear relaxation rate $\left(w_{1 n}\right.$ in Fig 2.7) determines the sequence repetition rate ${ }^{[49]}$. Unfortunately, this relaxation rate difficult to access by a magnetic resonance experiment and thus often remains unknown. The nuclear relaxation times can be several orders of magnitude longer with respect to the electron spin relaxation rate, particularly for experiments performed at very low temperatures and detecting low-gamma nuclei like ${ }^{2} \mathrm{H}[50$. Thus, the optimal relaxation interval for long-term signal averaging is on the order of the slow nuclear relaxation rate. Under these conditions, sequence repetition rates on the order of the fast electron spin relaxation rate are predicted to result in non-thermal equilibrium distribution of polarization at the end of a shot 26, 49] and thereby trigger a less efficient next ENDOR shot as illustrated in Fig. 2.7.

In practice, the ENDOR experiment is usually operated with repetition rates on the order of the electron spin, single shot acquisition per frequency point and stochastic rf frequency incrementation procedure rather than linear ${ }^{53]}$. These methods imply 
relaxation intervals of at least one scan duration before irradiating at the same rf frequency and the latter diminishes baseline distortions from rf-caused heating. Although a relief, these procedures not entirely overcome the issue for all applications, thus it is not surprising that there have been several publications focusing on approaches to partially overcome the current limitations of ENDOR spectroscopy $25,50,51,53,54$. The alternative ENDOR sequence developed in our research group is called coss-polarization edited ENDOR and will be discussed in the following section.

\subsection{Cross-polarization edited ENDOR spectroscopy}

The following section will provide an overview of the main concepts relevant for the understanding of the experimental eNCP and CP-ENDOR approach and the resulting beneficial spectra for proton nuclei rather than a comprehensive theoretical description. An extensive mathematical description can be found in the respective publications from our group 2527 .

The polarization transfer between electronic spins $S$ and nuclear spins $I$ via a crosspolarization (CP) transfer mechanism for the improvement of ENDOR spectroscopy was inspired by the CP approach in dynamic nuclear polarization (DNP) where it has been successfully applied for more than 50 years. In contrast to ENDOR spectroscopy, which aims to identify nuclei in the close vicinity of the paramagnetic center $(<2 \mathrm{~nm}$ to the radical) and determine the corresponding hyperfine interaction strength, the general DNP experiment is used to enhance the NMR signal of all nuclei of the sample and detect the NMR signal in a conventional NMR read-out scheme (Fig. 2.8).

A well-established approach for coherent polarization transfer between electron and nuclear spins relies on the Hartmann-Hahn (HH) method [55]. It is commonly used to transfer polarization from a high- $\gamma$ (see also Eq. 2.52), for instance protons, to a low- $\gamma$ target nucleus such as ${ }^{13} \mathrm{C}$ in spin systems with strong dipolar couplings as it is the usual case for solid-state NMR $[56$. The technique consists of a preparation pulse which generates transverse magnetization of the high- $\gamma$ nucleus followed by a long pulse (spinlock) simultaneously on both spins. At this stage polarization transfer occurs upon matching both spinlock field strengths $\omega_{1}^{1 \mathrm{H}}=\omega_{1}^{13 \mathrm{C}}$ driven by the dipolar coupling. 


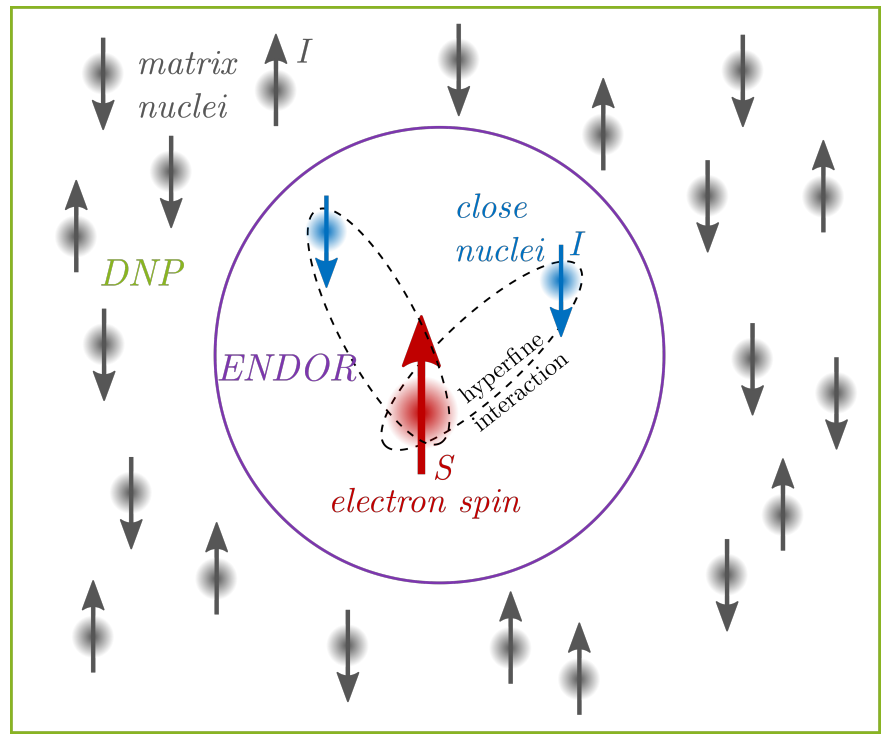

Figure 2.8: Illustration of the target nuclei types in ENDOR and DNP experiments. While ENDOR spectroscopy is suited to determine the hyperfine coupling strength between an electron spin an nearby nuclear spins ( $<2 \mathrm{~nm}$ to the radical), DNP is used to enhance the polarization of all magnetic nuclei of the sample. Thus, they can be detected with enhanced sensitivity in a conventional NMR experiment.

This approach becomes unfeasible for the application on electron and nuclear spins because the large discrepancy of their gyromagnetic ratios $\left(\gamma_{\mathrm{e}} / \gamma_{\mathrm{n}} \approx 660\right)$ causes a mismatch between the required mw and rf field strength. A partial solution to this limit called nuclear spin orientation via electron spin locking (NOVEL) proposed to spinlock only the electron spins such that the electron spin in the rotating frame is matched to the nuclear spin in the laboratory frame $\omega_{1}^{1 \mathrm{e}}=\omega_{1}^{0 \mathrm{n}}$. This was improved even further by the so called integrated solid effect (ISE) where a fast field sweep through the EPR line incorporates also off-resonance electron spins into the CP process. However, the application is limited to low static magnetic fields, for which the nuclear Larmor frequencies are on the order of the available mw field strength. Very recently, a NOVEL-based alternative which uses only a fraction of the microwave irradiation field strength compared to standard NOVEL has been proposed 57 . The technique requires a train of mw pulses substituting the long spinlock pulse with a particular spacing between each pulse (time-optimized DNP, TOP-DNP) and promising predicted applications at higher fields/frequencies. However, they are experimentally not yet demonstrated. Further, the authors 57 reported that TOP-DNP shows a longer polarization buildup time and therefore is less robust to $T_{2 \mathrm{e}}$ relaxation compared to NOVEL.

The underlying idea for the implementation of CP to ENDOR spectroscopy was proposed about 20 years ago, the so-called dressed-state solid effect (DSSE) [58. This approach 
uses a mw preparation pulse followed by a 90 degree phase shifted mw field interacting with the electron spin to create a so-called dressed state. Simultaneously, an additional rf irradiation pulse is used to create nuclear polarization by selective excitation of particular NMR transitions in the electron-spin dressed state, mediated by the hyperfine coupling. By that time, the description was derived for the specific DNP purpose where $A \ll \omega_{1 \mathrm{e}}$, but was later described in a more general framework of electronnuclear cross polarization (eNCP) $[59]$. The presented description 59 results in general eNCP matching conditions for strongly and for weakly coupled nuclei and predicts field-independent transfer efficiency. The proposed eNCP sequence [58, 59] is suited to monitor the polarization transfer to the nucleus as loss of electron spin polarization which will be discussed in Subsec. 2.4.1. A few years ago, the first part of this sequence was successfully merged with the ENDOR sequence a few years ago by researchers of our group 25 . The resulting electron-nuclear cross polarization edited ENDOR sequence (CP-ENDOR) introduces a new a polarization transfer concept for ENDOR spectroscopy as described in Subsec. 2.4.2. The general CP-ENDOR polarization scheme proposes a transfer of electron spin polarization to the nucleus in the CP step. Afterwards, the polarization is transferred back from the nuclear to the electron spin by the rf ENDOR pulse while monitoring the electron spin polarization. The following section summarizes the recently published eNCP and CP-ENDOR results on proton nuclei $2 5 \longdiv { 2 7 }$.

\subsubsection{CP matching conditions from ${ }^{1} \mathrm{H}$ eNCP experiments}

The eNCP experiment can be considered a pre-experiment from which the so-called CP matching conditions are determined. The eNCP pulse sequence consists of a selective $\pi / 2$ pulse creating electron spin coherence on one of the electron spin manifolds which is instantaneously spin locked by a $90^{\circ}$ phase shifted mw pulse. The spinlock pulse is applied with a constant, weak mw amplitude for a duration of several hundred microseconds. Simultaneously to the mw spinlock pulse, a frequency swept rf pulse with constant amplitude is applied on the target nuclear spin. In the last step of the sequence, a selective $\pi$ pulse with the same phase and constant amplitude as the initial $\pi / 2$ pulse is applied in order to create an electron-spin echo at the time point $2 \tau$ as illustrated in Fig. 2.9, A. At this stage, the loss of electron spin polarization due to $\mathrm{CP}$ as a function of the rf frequency is detected. Thus, a CP matching condition is observed as an intensity minimum in the eNCP spectrum, illustrated in Fig. 2.9, C. 


\section{A) eNCP sequence}

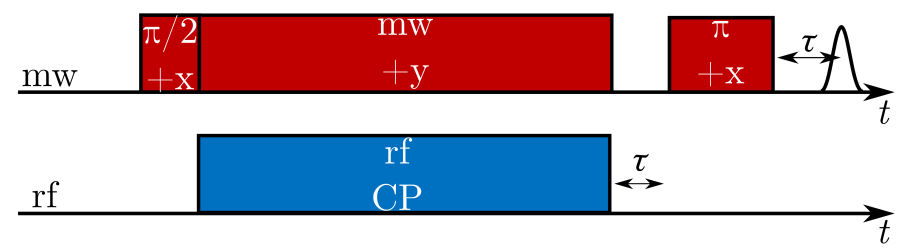

\section{B) Field-sweep ESE spectrum}

\section{C) eNCP spectrum}
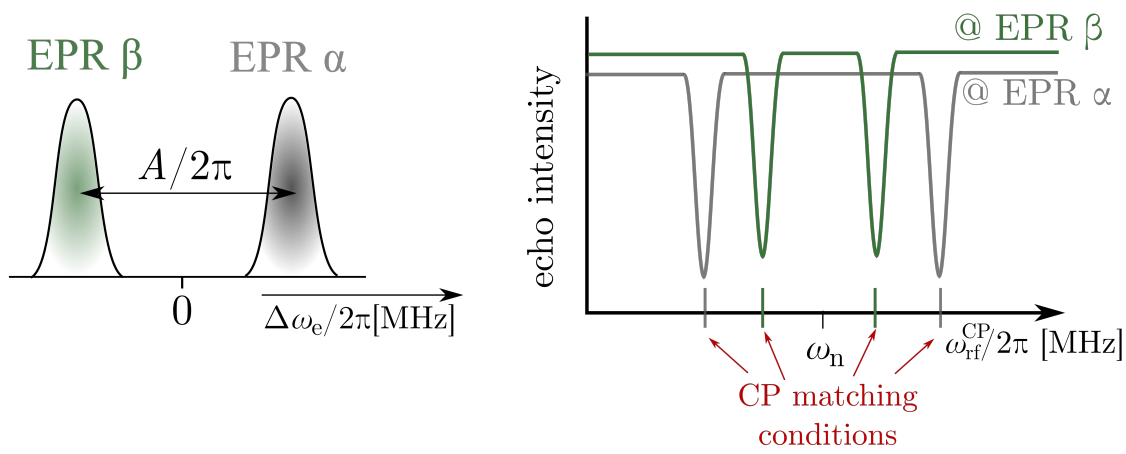

Figure 2.9: eNCP pulse sequence and idealized eNCP spectrum. $A$ ) The eNCP pulse sequence consists of a selective mw pulse followed by a 90 degree phase shifted long mw irradiation pulse. Concomitantly to the mw pulse, an frequency swept $\mathrm{rf}$ pulse is applied. A selective $\pi$-pulse refocuses the transverse magnetization to an echo. $B$ ) Idealized ESE spectrum of an $S=1 / 2, I=1 / 2, A>0$ spin system. EPR $\alpha$ and EPR $\beta$ refer to the EPR transitions between energy levels [1-3] and [2-4], respectively $C$ ) eNCP spectrum with two peaks (= matching conditions) symmetric around the nuclear Larmor frequency $\omega_{\mathrm{n}}$ observed from selective excitation of an EPR transition. Adapted from $[52]$.

Following the formalism presented in 25$]$, CP between an electron spin $S=1 / 2$ coupled to a proton nuclei $I=1 / 2$ occurs when a matching conditions according to

$$
\Delta \omega_{\mathrm{n}}^{\mathrm{CP}}= \pm \frac{1}{2}\left(\omega_{\alpha} \pm \omega_{\beta}\right)
$$

is satisfied, where $\omega_{\alpha}$ and $\omega_{\beta}$ are the effective fields of the two electron spin manifolds defined as $\omega_{\alpha}=\sqrt{\left(-\Delta \omega_{\mathrm{e}}+\frac{A}{2}\right)^{2}+\omega_{1 \mathrm{e}}^{2}}$ and $\omega_{\beta}=\sqrt{\left(-\Delta \omega_{\mathrm{e}}-\frac{A}{2}\right)^{2}+\omega_{1 \mathrm{e}}^{2}}$. In principle, four matching conditions according to Eq. 2.53 are predicted, but considering selective EPR excitation, only two matching conditions for each EPR transition are observed. They were assigned from single crystal investigations as presented in 27$]$ (Fig. 2.9, B). During the spinlock period $t_{\mathrm{CP}}$ the electron spins are in a dressed state and interact with the nuclear spins in the so-called bare state. Thus, the electron spins are quantized along the effective field $\omega_{\alpha} / \omega_{\beta}$ and the nuclear spins remain quantized along the $z$-axis of the laboratory frame as illustrated in Fig. 2.10. 


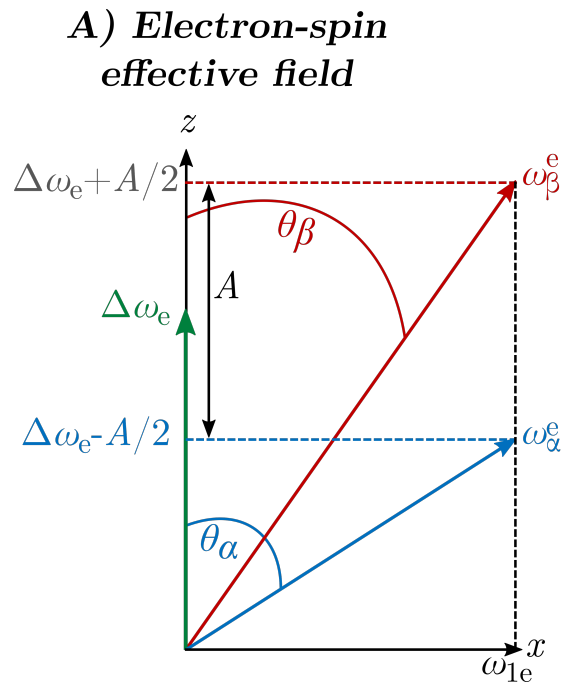

\section{B) Nuclear-spin effective field}

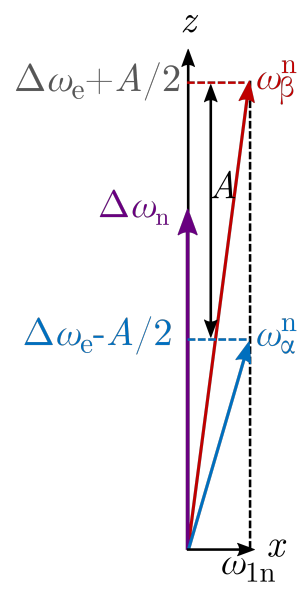

Figure 2.10: Electron- and nuclear-spin effective fields during the eNCP transfer. A) Electron spins in the tilted frame are quantized along the effective field directions $\omega_{\alpha}^{\mathrm{e}}, \omega_{\beta}^{\mathrm{e}}$, which depend on $\Delta \omega_{\mathrm{e}}, A$ and $\omega_{1 \mathrm{e}} . B$ ) The effective field of the nuclear spins $\omega_{\alpha}^{\mathrm{n}}, \omega_{\beta}^{\mathrm{n}}$ remains along the $z$-axis of the laboratory frame during the CP transfer. Figure adapted from $[52$.

The assumption holds as long as $\omega_{1 \mathrm{n}}$ (usually $\mathrm{kHz}$ ) is small compared to $A$ (usually $\mathrm{MHz}$ ) which is a common case in ENDOR experiments. The corresponding frame of reference for the description of the eNCP process is called tilted frame and it depends on a combination of $\Delta \omega_{\mathrm{e}}, A$ and $\omega_{1 \mathrm{e}}$. The Hamiltonian $\mathcal{H}^{\prime}$ in this tilted frame gets the form ${ }^{3}$

$$
\tilde{\mathcal{H}}^{\prime}=\left[\begin{array}{cccc}
-\frac{\omega_{\alpha}}{2}-\Delta \omega_{\mathrm{n}} & \omega_{1 \mathrm{n}} \cos \left(\frac{\theta_{\beta}}{2}-\frac{\theta_{\alpha}}{2}\right) & 0 & \omega_{1 \mathrm{n}} \sin \left(\frac{\theta_{\beta}}{2}-\frac{\theta_{\alpha}}{2}\right) \\
\omega_{1 \mathrm{n}} \cos \left(\frac{\theta_{\beta}}{2}-\frac{\theta_{\alpha}}{2}\right) & -\frac{\omega_{\beta}}{2}+\Delta \omega_{\mathrm{n}} & \omega_{1 \mathrm{n}} \sin \left(\frac{\theta_{\beta}}{2}-\frac{\theta_{\alpha}}{2}\right) & 0 \\
0 & \omega_{1 \mathrm{n}} \sin \left(\frac{\theta_{\beta}}{2}-\frac{\theta_{\alpha}}{2}\right) & +\frac{\omega_{\alpha}}{2}-\Delta \omega_{\mathrm{n}} & \omega_{1 \mathrm{n}} \cos \left(\frac{\theta_{\beta}}{2}-\frac{\theta_{\alpha}}{2}\right) \\
\omega_{1 \mathrm{n}} \sin \left(\frac{\theta_{\beta}}{2}-\frac{\theta_{\alpha}}{2}\right) & 0 & \omega_{1 \mathrm{n}} \cos \left(\frac{\theta_{\beta}}{2}-\frac{\theta_{\alpha}}{2}\right) & +\frac{\omega_{\beta}}{2}+\Delta \omega_{\mathrm{n}}
\end{array}\right]
$$

where $\theta_{\alpha}$ and $\theta_{\beta}$ are the turning angles which connect the laboratory frame with the tilted frame for each electron spin manifold as illustrated in Fig. 2.10. From the Hamiltonian in the tilted frame it can be derived that a set of off-diagonal elements proportional to the rf field amplitude $\omega_{1 \mathrm{n}}$ connects pairs of energy levels in the tilted frame. Accordingly, $\mathrm{CP}$ is driven when the energy difference between such connecting diagonal elements becomes on the order of $\omega_{1 \mathrm{n}}$. In the formalism published on the protons ${ }^{25}$ the time evolution of the diagonal elements of the density matrix during CP in the tilted frame

\footnotetext{
${ }^{3}$ The tilde on top of the $\mathcal{H}$ refers to an ordering of energy levels introduced to simplify the CP-ENDOR description, thus non-following the conventional ordering as for instance given in 29$]$. The respective transformation formalism for the deuterium nuclei studied in this theses is given in Appendix $\bar{C}$.
} 
was reported to oscillate between two CP-matched levels until their populations are equalized. At the end of the CP transfer, nuclear polarization is generated and will be further used for ENDOR spectroscopy. Precisely, the eNCP step replaces the selective $\pi$ preparation pulse of the Davies ENDOR sequence as will be described in the following section. Though before introducing the CP-ENDOR sequence, the eNCP experiment on powder/frozen solution systems will be discussed briefly.

The above description represents the behavior of a two spin system under isotropic conditions, such as a single crystal 27. However, most of the EPR/ENDOR experiments are performed on power samples or frozen solutions where multiple molecules are randomly oriented (see Subsec. 2.3.4). Consequently, the appearing $g$ - and $A$-anisotropy effects will broaden the observed eNCP spectrum for two reasons (illustrated in Fig. 2.11). Firstly, resonances of different spin packets are shifted to slightly different Larmor frequencies and will overlap, thus selective excitation of only one EPR transition becomes unfeasible. Secondly, the excited spin packets show various $A$ due to hf anisotropy which results in a different $\Delta \omega_{\mathrm{n}}^{\mathrm{CP}}$ for each $A\left(\Delta \omega_{\mathrm{n}}^{\mathrm{CP}}\right.$ depends on $A$ according to Eq. 2.53). Accordingly, when the matching condition is set to a fixed rf offset value, only nuclear spins coupled with a distinct hyperfine coupling $A$ are polarized in the CP process. This selective polarization transfer has to be taken into account for CP-ENDOR experiments performed on powder or frozen solution samples as will be discussed in the next section. 
A) Field-sweep ESE spectrum

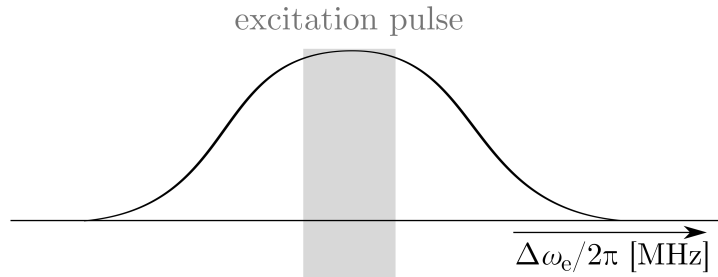

individual spin packets
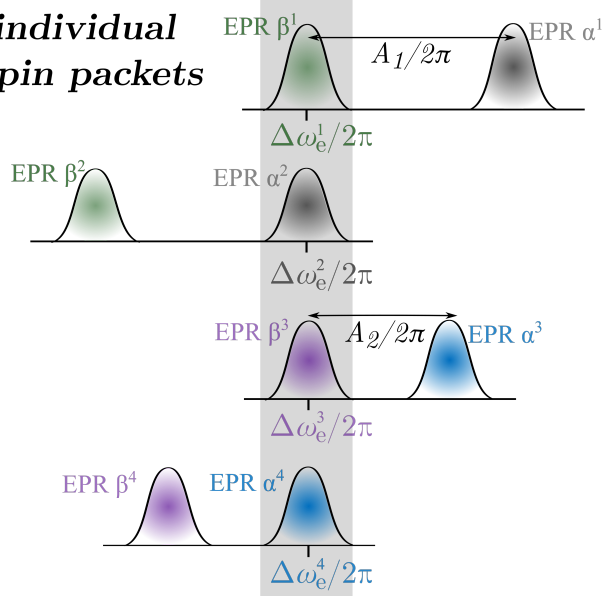

B) eNCP spectrum

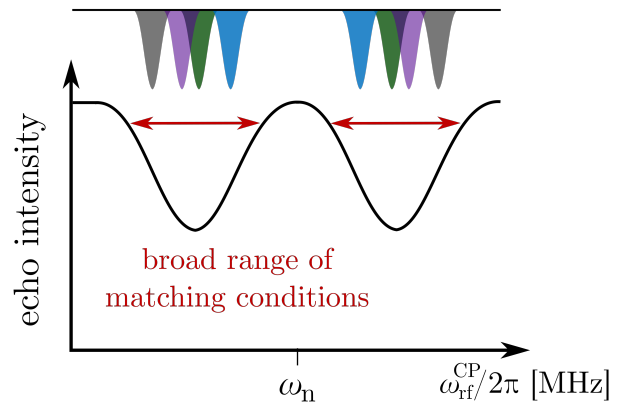

Figure 2.11: Illustration of a powder pattern eNCP spectrum. $A$ ) Resonances of different spin packets $i$ are shifted to slightly different Larmor frequencies $\omega_{\mathrm{e}}^{i}$ and will overlap and result in a broad EPR line. Selective excitation of only one EPR transition from a broad EPR line becomes unfeasible $B$ ) All excited EPR transitions (grey area) contribute to the eNCP spectrum and instead of sharp peaks from the individual spin packets, broad matching condition ranges (red arrows) are obtained.

\subsection{2. ${ }^{1} \mathrm{H}$ CP-ENDOR spectroscopy}

In the CP-ENDOR sequence shown in Fig. $2.12 \mathrm{~A}$, the selective $\pi$ preparation pulse from Davies ENDOR is replaced by the first part of the eNCP sequence (compare with Fig. 2.3). The sequence can be divided in three parts. 1) The CP preparation part, where electron spin polarization is transferred to the nucleus, 2) the ENDOR part, where an NMR transition is driven and thereby polarization is transferred from the nucleus to the electron spin and 3) the read-out part in order to detect the change in electron polarization (= ENDOR effect) as a function of the rf ENDOR pulse. The polarization transfer during the CP-ENDOR sequence in rotating and tilted frame is illustrated in Fig. 2.12, B, on basis of the theoretical predictions presented in 25]. At the beginning of the sequence, the four energy levels of a $S=1 / 2, I=1$ spin system, $A<0$ system are populated according to Boltzmann statistics. The amount of population is represented by grey balls of different size. After a selective $\pi / 2$ mw excitation pulse, for instance on the EPR [1-3] transition, one pair of energy levels is split by the mw field strength $\omega_{1 \mathrm{e}}$ and the other by $A$ when described in the tilted frame. 
A) CP-ENDOR sequence

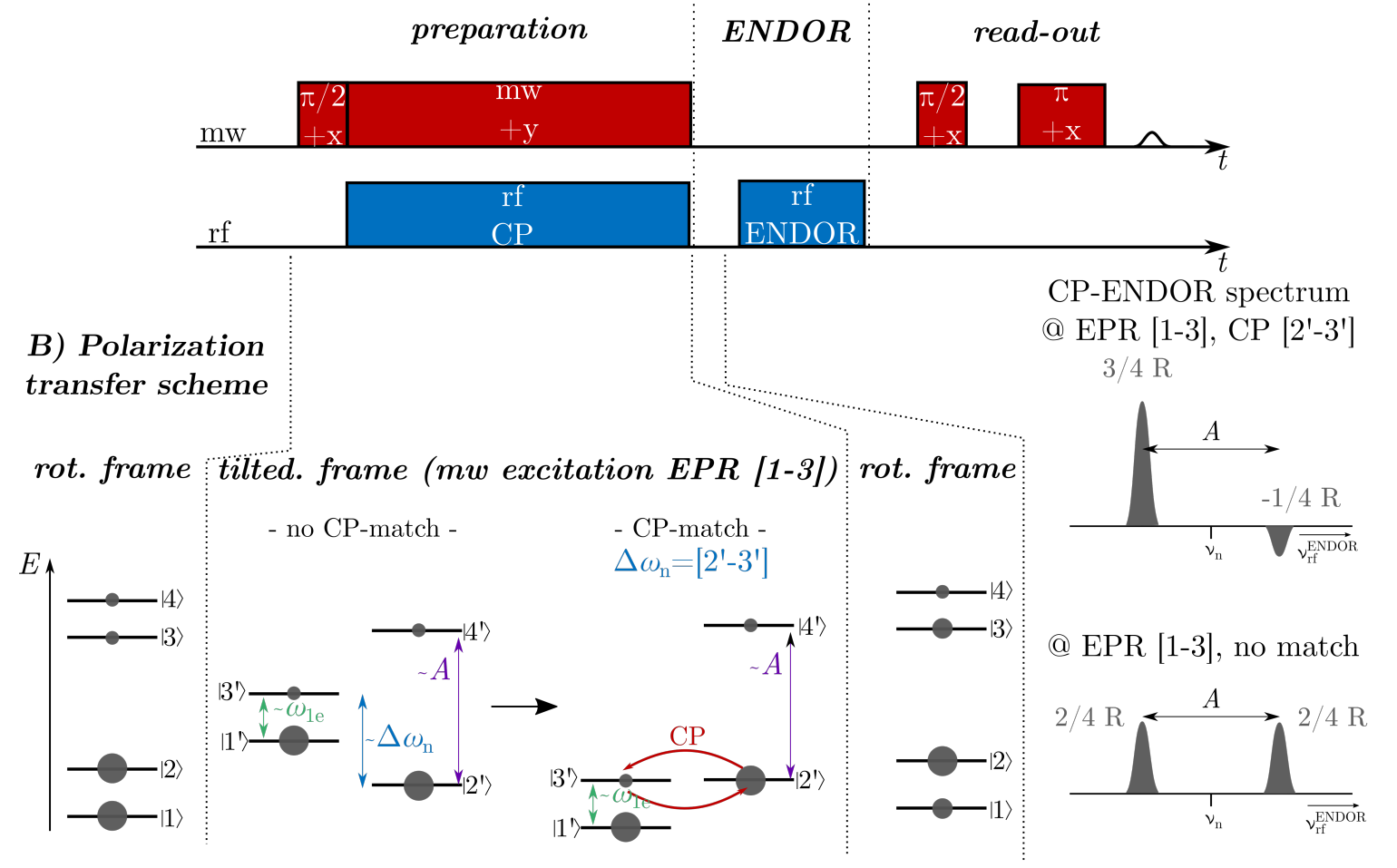

Figure 2.12: CP-ENDOR pulse sequence, polarization transfer scheme and idealized CP-ENDOR spectrum. Energy level scheme depicted for a $S=1 / 2, I=1 / 2, A<0$ spin system and $\omega_{1 \mathrm{e}} \leqslant A$. A) During the preparation step a selective $\pi / 2$ preparation pulse selectively excites one EPR transition followed by a long mw spinlock and rf irradiation pulse. The rf CP frequency is set to satisfy a matching condition. Afterwards, a frequency-swept ENDOR rf pulse followed by a mw echo detection sequence is applied. B) Polarization transfer scheme in the rotating and tilted frame for selective excitation of EPR transition [1-3]. The amount of population is represented by grey balls of different size. The energy level splitting in the tilted frame is given by $A, \omega_{1 \mathrm{e}}$ and $\Delta \omega_{\mathrm{n}}$. CP polarization transfer is illustrated for polarization transfer between levels $\left[2^{\prime}-3^{\prime}\right]$. The predicted CP-ENDOR spectrum shows asymmetric peaks. Figure adapted from 52 .

The tilted frame description is valid as long as the long mw pulse is applied. During that period, two of the energy levels in the tilted frame are degenerate when a matching condition is satisfied. After the CP-step, back in the rotating frame, a polarization pattern corresponding to nuclear polarization is created. Finally, the frequency-swept rf ENDOR pulse will change polarization of a NMR transition and the ENDOR spectrum with CP characteristic asymmetric ENDOR peaks will be obtained (Fig. 2.12, B, right). The asymmetry is due to the fact that at the end of the CP-step the NMR manifold are oppositely polarized with respect to each other. The predicted CP-ENDOR intensities for a $S=1 / 2, I=1 / 2, A>0$ spin system are $-1 / 4 \mathrm{R}: 3 / 4 \mathrm{R}$ for the shown example 25,27 . The constant $R$ is the polarization factor and is proportional to 
the projection of the magnetization vector onto the effective field and to Boltzmann population. Comprehensive calculations of CP-ENDOR intensities can be found in 25, 27. and are summarized for all relevant combinations of selective EPR excitation and CP matching condition in Tab. 5.4.

Table 2.1: Analytically calculated CP-ENDOR intensities for an electron spin-1/2 coupled to a proton. The intensities are listed for a $S=1 / 2, I=1 / 2$, $A<0$ spin system as published in 27 .

\begin{tabular}{c||c|c|c|c}
\multicolumn{1}{c||}{} & \multicolumn{2}{c|}{ EPR $\alpha,[1-3]$} & \multicolumn{2}{c}{ EPR $\beta,[2-4]$} \\
& NMR & NMR & NMR & NMR \\
& {$[1-2]$} & {$[3-4]$} & {$[1-2]$} & {$[3-4]$} \\
\hline \hline $\mathrm{CP}\left[1^{\prime}-4^{\prime}\right]$ & $-1 / 4 \mathrm{R}$ & $3 / 4 \mathrm{R}$ & $2 / 4 \mathrm{R}$ & $2 / 4 \mathrm{R}$ \\
$\mathrm{CP}\left[2^{\prime}-3^{\prime}\right]$ & $3 / 4 \mathrm{R}$ & $-1 / 4 \mathrm{R}$ & $2 / 4 \mathrm{R}$ & $2 / 4 \mathrm{R}$ \\
$\mathrm{CP}\left[1^{\prime}-2^{\prime}\right]$ & $2 / 4 \mathrm{R}$ & $2 / 4 \mathrm{R}$ & $-1 / 4 \mathrm{R}$ & $3 / 4 \mathrm{R}$ \\
$\mathrm{CP}\left[3^{\prime}-4^{\prime}\right]$ & $2 / 4 \mathrm{R}$ & $2 / 4 \mathrm{R}$ & $3 / 4 \mathrm{R}$ & $-1 / 4 \mathrm{R}$
\end{tabular}

Although it might seem paradox at first sight, an ENDOR spectrum is observed even if no matching condition is fulfilled during the CP step. In such case, the selective $\pi / 2$ pulse together with the long mw irradiation pulse create for instance a $S_{x} I^{\alpha}-S_{z} I^{\beta}$ term denoted in the product operator formalism (see Subsec. 2.3.2). This refers to electron spin polarization $-S_{z} I^{\beta}$ of one electron spin manifold, however no electron spin polarization but coherence of the other manifold $S_{x} I^{\alpha}$ (Fig. 2.13, A $\rightarrow$ B). In such case, the rf pulse on-resonant with a NMR transition will re-create electron spin polarization on $S_{x} I^{\alpha}$. The resulting difference in electron polarization as a function of the $r f$ frequency is detected by the selective read-out echo detection (Fig. 2.13, C).

A)

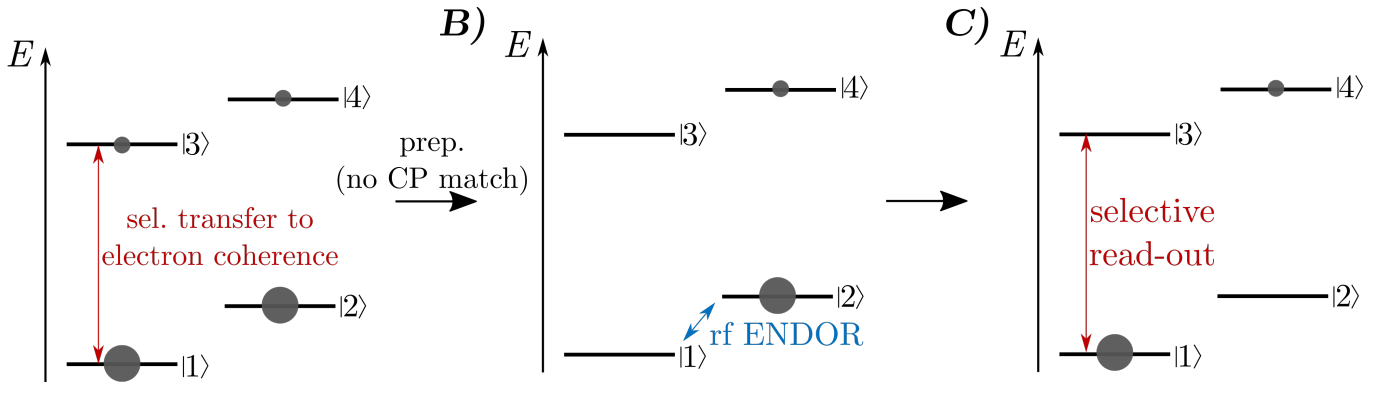

Figure 2.13: Illustration of polarization transfer for a non-matched CPENDOR case. A) The selective mw pulse on the EPR [1-3] transition creates $B$ ) electron spin coherence on the excited manifold and leaves the other manifold unperturbed $\left.\left(S_{x} I^{\alpha}-S_{z} I^{\beta}\right) . C\right)$ Electron spin polarization is re-obtained and selectively detected when the rf ENDOR pulse is on-resonant with an NMR transition (e.g. NMR [1-2]). 
Furthermore, the product operator formalism allows to point out some key differences between CP-ENDOR and Davies ENDOR, summarized in Fig. 2.14. The product operators at the end of the preparation step and after the ENDOR step for each sequence are given by

$$
\begin{aligned}
& \text { Davies ENDOR : } 2 S_{z} I_{z} \stackrel{\text { rf ENDOR }}{\longrightarrow} I_{z} \\
& \text { CP-ENDOR : } \quad I_{z} \stackrel{\text { rf ENDOR }}{\longrightarrow} 2 S_{z} I_{z}
\end{aligned}
$$

and identify the CP-ENDOR sequence as reverse of the Davies ENDOR sequence. This has two major consequences. In CP-ENDOR, the detected electron spin polarization is small unless the rf ENDOR pulse is on-resonant with an NMR transition. Thus, the ENDOR effect in CP-ENDOR is obtained as a gain in echo intensity. This is contrary to Davies ENDOR where the observed echo vanishes when an NMR transition is driven.

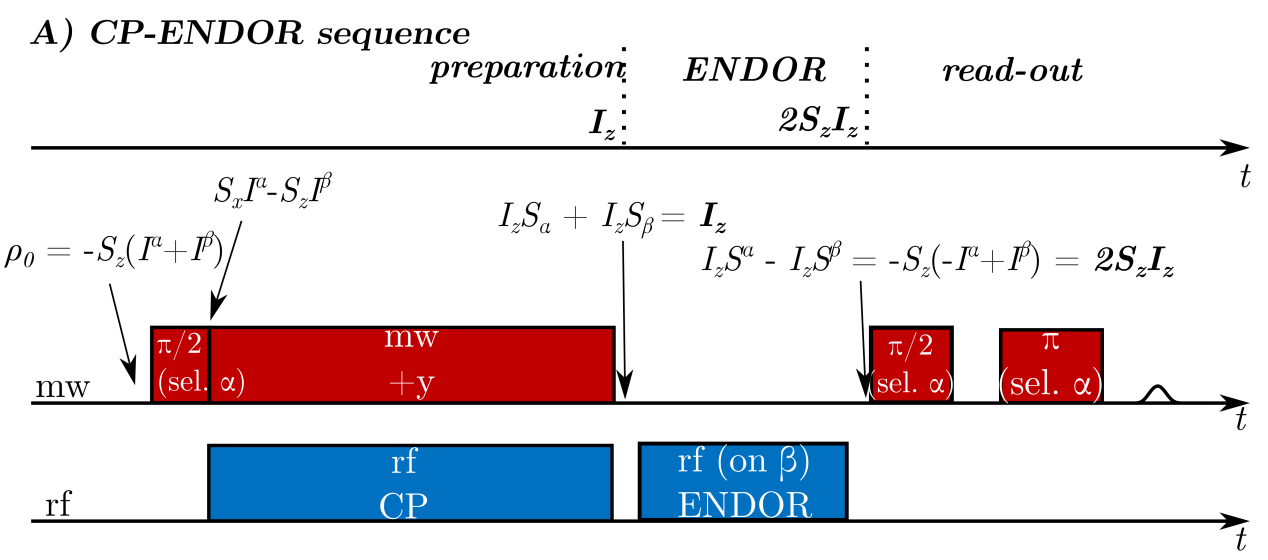

B) Davies ENDOR sequence

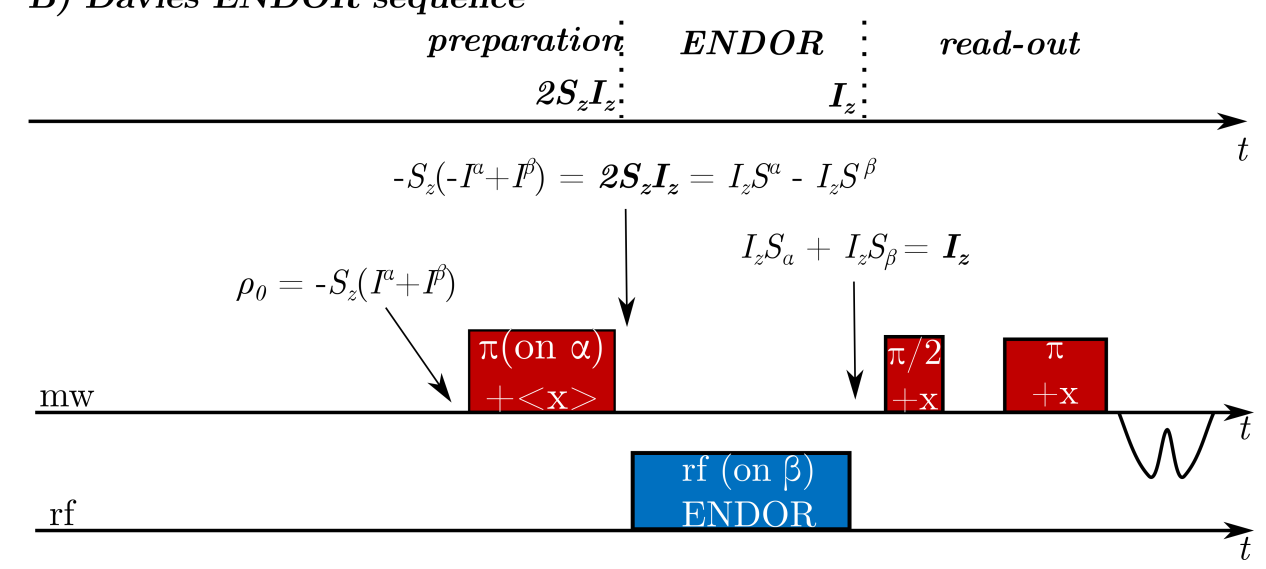

Figure 2.14: CP-ENDOR sequence and Davies ENDOR sequence described by the product operator formalism. A) At the end of the preparation step $I_{z}$ is generated, which is transferred to $2 S_{z} I_{z}$ by the ENDOR rf pulse. The ENDOR effect is observed as gain in echo intensity. $B$ ) Reverse to the CP-ENDOR sequence, in Davies ENDOR the preparation step creates $2 S_{z} I_{z}$ and $I_{z}$ is observed after the ENDOR rf pulse. The ENDOR effect is observed as vanishing echo intensity. 
Finally, this CP-ENDOR polarization scheme results in a polarization pattern at the end of the sequence which does not solely rely on the nuclear relaxation rate in order to re-obtain thermal equilibrium, but follows a bi-exponential decay attributed to the electron- and nuclear-spin relaxation rate 26 . Consequently, the CP ENDOR experiment can be acquired with a repetition time on the order of the electron spin relaxation time in contrast to Davies ENDOR. Optimal performance in Davies ENDOR requires waiting times on the order of the nuclear-spin lattice relaxation. This is a major benefit of the CP-ENDOR experiment and has been investigated by numerical calculations as well as comparative experiments 26,27 .

In particular, the presented $\mathrm{S} / \mathrm{N}$ improvements observed for ENDOR spectra on a stable tyrosyl radical in the ribonucleotide reductase enzyme from $E$. coli ${ }^{27}$ demonstrated the enhanced performance of CP-ENDOR spectroscopy. In this study, the ENDOR performance comparison at $94 \mathrm{GHz} / 3.4 \mathrm{~T}$ and low temperature $(T=5 \mathrm{~K})$ revealed $\approx 3.5$ times higher ENDOR signal intensities, which corresponds to $\approx 12$ times less averaging time.

However, the application of CP-ENDOR on powder and frozen solution samples requires an additional experimental modification. Specifically, the broadening of the EPR line and the resulting distribution of the matching conditions needs to be considered (see Fig. 2.11). The distribution of matching conditions is taken into account by acquiring CP-ENDOR spectra in a 2D-fashion 25, 27. Each scan of the experiment (= sweep of the rf ENDOR frequency) is recorded at a fixed CP rf offset frequency. The CP rf offset frequency is incremented ( $y$-dimension) after each ENDOR scan, such that after several increments the entire matching condition range is covered. The particular CP rf offset will result in an asymmetric ENDOR spectrum ( $x$-dimension) and the enhancement/asymmetry pattern differs for different CP rf offsets values. Accordingly, the 2D approach results in a so-called 2D CP-ENDOR spectrum (shown as landscape and/or contour plots) (see for instance Fig. 7.4 in Chapter 7). In order to re-established the common ENDOR line shape and compare the experiment with the standard ENDOR experiments, all CP-ENDOR spectra with different CP rf offsets are summed (see also Fig. 7.4 in Chapter 7). Therefore, CP-ENDOR experiments on powder and frozen solution samples are usually recorded at several different CP rf-offsets frequencies in order to ensure that the $\mathrm{CP}$ effect contributes to each hyperfine line ${ }^{27}$ and artefacts due to unbalanced CP enhancements can be excluded. 


\subsection{E.coli ribonucleotide reductase}

Ribonucleotide reductases (RNRs) comprises a class of enzymes which catalyze the reduction of nucleotides to 2'-deoxynucleotides in all organisms and thereby provide the precursors needed for DNA synthesis and repair 60 62]. The Escherichia coli (E. coli) RNR uses a reversible radical transfer (rt) mechanism which serves as a paradigm to study proton-coupled electron transfer (PCET) processes in biological transformations [63, 64]. Over the past decades, pioneering research aiming at structural and mechanistic elucidation of ribonucleotide reductases was and still is pursued 65-70. A fundamental part of the experiments presented in this thesis were performed on a tyrosyl radical intermediate in E. coli RNR. Although a structural identification was the aim, the main focus of these experiments has to be seen in a spectroscopic context, rather than a biological one. Therefore, the following section provides only a very brief overview about the E. coli RNR enzyme structure and mechanism as well as the strategy for the formation of the radical intermediates studied in this project. For further reading, numerous reviews provide a good starting point 7174 .

E. coli RNR is composed of two homodimeric subunits $\alpha 2$ and $\beta 2$ (Fig. 2.15, top) which form the active $\alpha 2 \beta 2$ complex upon binding of substrate and allosteric effector ${ }^{75}$. For each catalytic turnover, $\beta 2, \alpha 2$, substrate and effector must interact, reducing $\mathrm{Y}_{122} \bullet$ in $\beta 2$ and oxidizing $\mathrm{C}_{439}$ to a thiyl radical in the active side of $\alpha 2$ [76, 77]. However, an $\mathrm{X}$-ray structure of the active enzyme is still not available because the transient $\alpha 2 \beta 2$ complex could not be crystallized to so far. Instead, an in silico model of the active complex on the basis of shape complementary of the individual X-ray structures of the subunits was firstly proposed by Uhlin and Eklund, referred to as docking model shown in Fig. 2.15 (bottom) 66, 78. Through the years, this docking model has been established as a valuable representation of the active RNR structure by several biophysical and biochemical investigations 63, 69, 74, 79, 80,

From the docking model it was proposed that $\mathrm{Y}_{122} \bullet$ and the catalytic side are about $35 \AA$ apart which resulted in a proposed electron transfer over an unprecedented distance of $>35 \AA$. This transfer, however, is not a one step transfer but a reversible half-side hopping mechanism involving at least five redox-active amino acids $\frac{79,81}{81}$. Three of them were identified as tyrosyl residues, assigned to $\mathrm{Y}_{356}$ in $\beta 2$ and $\mathrm{Y}_{731}$ and $\mathrm{Y}_{730}$ in $\alpha 2$ [16, 17, 63, 78. They were identified to be essential from phenylalanine mutants of $Y_{730^{-}}$ $\alpha 2, \mathrm{Y}_{731^{-}} \alpha 2$ and a $\mathrm{Y}_{356^{-}} \beta 2$ which showed no enzyme activity while $\mathrm{X}$-ray crystallography validated the unaltered structure of the mutated subunits $\left.{ }^{74,} 82\right]$. 


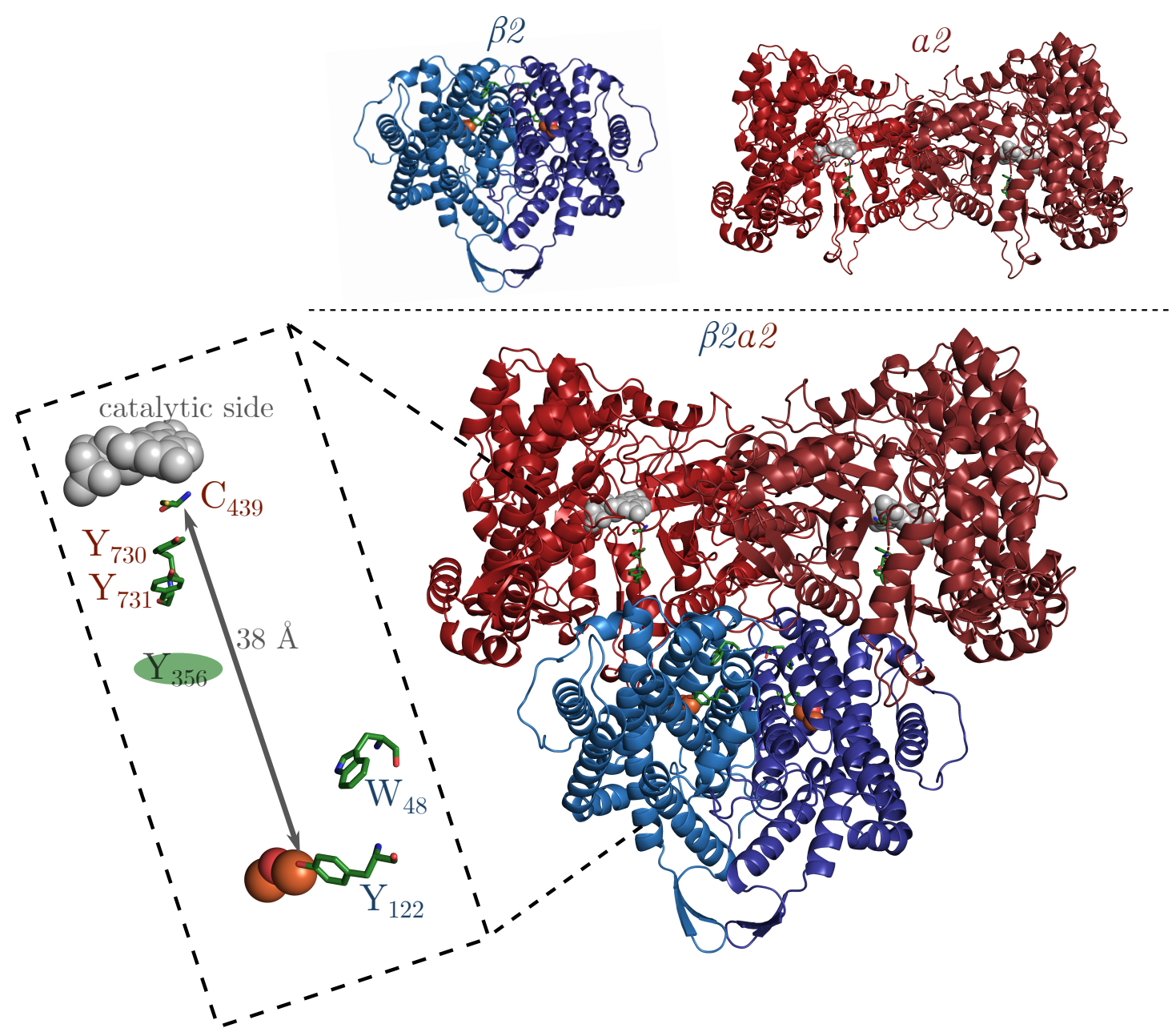

Figure 2.15: Docking model of the E. coli $\alpha 2 \beta 2$ complex. The complex consists of two homodimeric subunits $\alpha 2$ (red, PDB ID: 4R1R) and $\beta 2$ (blue, PDB ID: 1MXR). The inset shows the redox-active amino acids involved in the PCET $\left(\mathrm{Y}_{122} \rightleftharpoons[\mathrm{W} 48\right.$ ? $] \rightleftharpoons \mathrm{Y}_{356}$ in $\beta 2$ to $\mathrm{Y}_{731} \rightleftharpoons \mathrm{Y}_{730} \rightleftharpoons \mathrm{C}_{439}$ in $\left.\alpha 2\right)$. The exact spatial localization of $\mathrm{Y}_{356}$ is unknown because it is part of the flexible C-terminal tail (35 amino acids) of $\beta 2$. The question mark at W48 indicates that there is no direct evidence for its role in PCET. Figure adapted from 63 .

The radical transfer involving these intermediates is proposed to occur in a PCET mechanism, thereby preventing the formation of highly energetic, charged intermediates $16,17,74,83$. Of particular interest is the understanding of the radical transfer mechanism between the two subunits which involves residues $\mathrm{Y}_{356}$ in $\beta 2$ and $\mathrm{Y}_{731}$ in $\alpha 2$. This connection is particularly puzzling because $\mathrm{Y}_{356}$ is located in the flexible C-terminal tail (35 amino acids) which is unresolved in the X-ray structure of the $\beta 2$ subunit, thus, lacking in the docking model 66, 78.

The mechanistic details of electron and proton pathways have been investigated by EPR spectroscopy using high field/frequency EPR, pulsed electron-electron double resonance (PELDOR) and/or ENDOR ${ }^{16}-18,69$. In particular, EPR studies on site-specifically 
incorporated unnatural amino acids as spin traps have become a powerful tool to gain mechanistic insights via distances measurements 18,69 and hydrogen-bonding network investigations $16,17,19$. These unnatural amino acid spin traps were developed by the research group of JoAnne Stubbe (Massachusetts Institute of Technology, Cambridge). The necessity of altering the energy landscape of the PCET arises from the fact that the rate limiting step is a conformational change prior to rapid nucleotide reduction and rapid tyrosine oxidation 84 . Furthermore, the radical transfer follows a thermodynamic uphill pathway $(>200 \mathrm{mV})$ [79. 85 , schematically shown in Fig. 2.16. Thus, spectroscopic detection of pathway intermediates in wild-type enzyme becomes unfeasible.

One major group of spin traps are $\mathrm{NH}_{2} \mathrm{Ys}_{\mathrm{s}}$ incorporated at positions $\mathrm{Y}_{356}$ in $\beta 2^{[85]}$ as well as $\mathrm{Y}_{731}$ and $\mathrm{Y}_{730}$ in $\alpha 2^{79}$ which provide a reduced redox potential at the respective residue, illustrated for the $\mathrm{NH}_{2} \mathrm{Y}$ mutation at $\mathrm{Y}_{731}$ in Fig. 2.16.

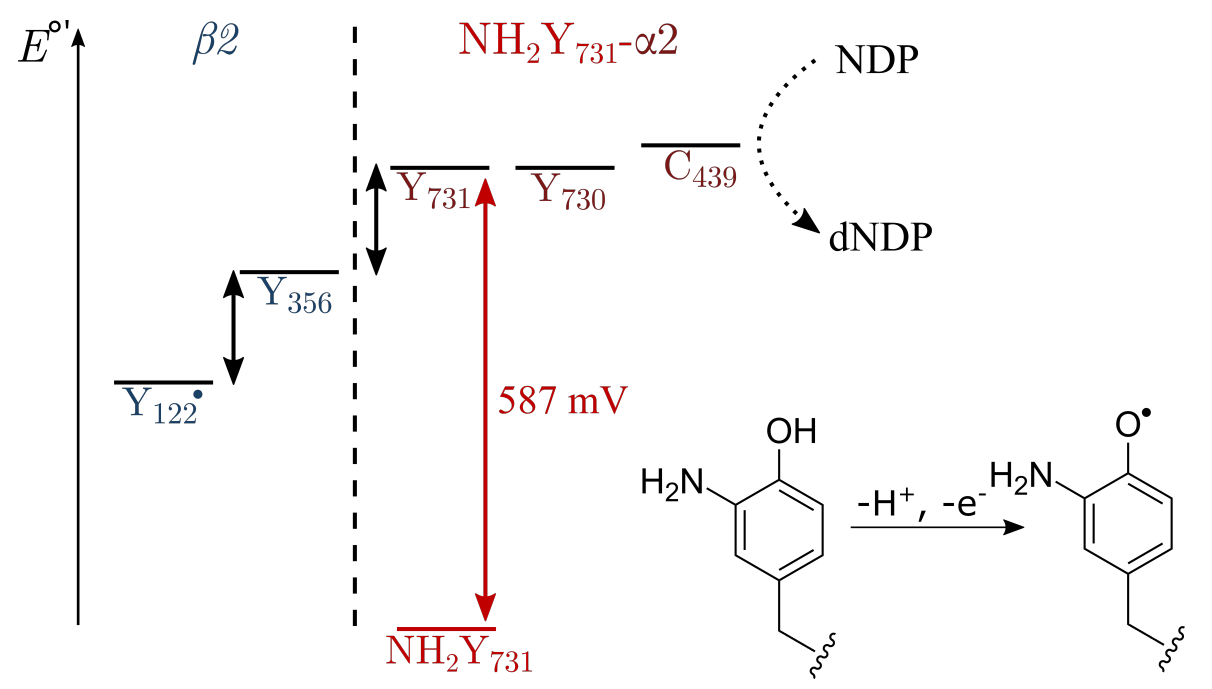

Figure 2.16: Schematic thermodynamic landscape for the rt pathway in $\boldsymbol{E}$. coli RNR. The forward rt transfer follows a thermodynamic uphill pathway. Site-specific incorporation of $\mathrm{NH}_{2} \mathrm{Y}$ at $\mathrm{Y}_{356}$ in $\beta 2$ or $\mathrm{Y}_{731}$ and $\mathrm{Y}_{730}$ in $\alpha 2$ reduce the redox potential at the respective mutation position, illustrated for $\mathrm{NH}_{2} \mathrm{Y}$ at $\mathrm{Y}_{731}$. Once the catalytic process is initialized and the radical approaches the modified amino acid, $\mathrm{NH}_{2} \mathrm{Y}$ is reduced to $\mathrm{NH}_{2} \mathrm{Y}^{\bullet}$. Figure adapted from 86 .

By combining $\mathrm{NH}_{2} \mathrm{Y}-\alpha 2 / \beta 2$ subunit with the complementary subunit, as well as substrate (CDP) and effector (ATP), the radical can be trapped kinetically (lifetime of a few seconds). The formed radicals have been in focus of several EPR studies from which not only the docking model was confirmed but also detailed insights into the local PCET mechanism were obtained $16,19,79]$.

In general, the $\mathrm{NH}_{2} \mathrm{Y}$ spin-trap functionality is based on its reduced redox potential when compared to $\mathrm{Y}$. Specifically, a sink of $587 \mathrm{mV}$ in the rt pathway at $\mathrm{NH}_{2} \mathrm{Y}_{731^{-}}$ 
$\alpha 2$ was determined from cyclic voltammetry studies ${ }^{[86}$, certainly enough to block further rt (see Fig. 2.16). However, protein environments can tune redox potentials through conformational changes 87 . Density functional theory (DFT) calculations of a 2-aminophenol [86] predicted a possible increase of the redox potential by 190-390 $\mathrm{mV}$ upon tilting of the amino group. This change could be enough to still enable rt to the next amino acid residue. For answering the question of the $\mathrm{NH}_{2} \mathrm{Y}$ radical intermediate conformation, orientation-selective ENDOR spectroscopy is in principle well suited. Although the $\mathrm{NH}_{2} \mathrm{Y}$ mutation strategy has been available for more than 10 years and published DFT calculations suggested a planar structure of the $\mathrm{NH}_{2}$ group in 2015 already, an unambiguous spectroscopic evidence was only recently provided in conjunction with the results obtained in this thesis 86 . 



\section{Structure and scope of the thesis}

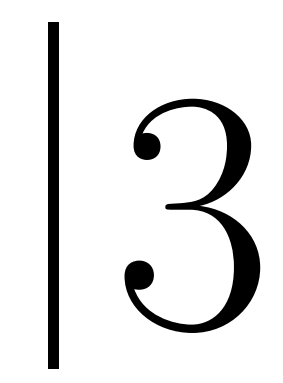

High-field ENDOR spectroscopy is an important EPR technique for the determination of hyperfine couplings between paramagnetic centers and the magnetic nuclei in the close vicinity. Deuterium ENDOR studies of biomolecules in combination with $\mathrm{H}_{2} \mathrm{O} \rightarrow \mathrm{D}_{2} \mathrm{O}$ solvent exchange are of particular interest. The buffer exchanged deuterium hyperfine couplings are probed at ENDOR frequency ranges well-separated from those of nonexchangeable protons of the biomolecule's backbone ${ }^{20 \mid 22]}$. Such ${ }^{2}$ H ENDOR studies are, for instance, essential for the elucidation of hydrogen-bond environments in systems as complex as enzymes.

The deuterium, however, represent a challenging target nuclear spin for ENDOR spectroscopy. Its low gyromagnetic ratio $\left(\gamma_{\mathrm{H}} / \gamma_{\mathrm{D}} \approx 6.5\right)$ is the origin of very weak hyperfine interactions which result in small hyperfine couplings. The established ENDOR techniques are associated with either low sensitivity (Davies ENDOR) or line shape distortions (Mims ENDOR) regarding the detection of small hyperfine couplings. Additionally, the manipulation of electron and nuclear spins, without taking into account of the fact that the nuclear-spin relaxation times are significantly longer than the electron-spin relaxation times, results in nuclear saturation and ENDOR signal loss during long-term signal acquisition. The CP-ENDOR sequence introduces a new polarization transfer mechanism into ENDOR spectroscopy 25]. Its improved sensitivity and robustness towards nuclear saturation have been demonstrated for the detection of large proton hyperfine couplings $26,27$.

The objective of this thesis is to improve the sensitivity for the detection of small deuterium hyperfine couplings with ${ }^{2} \mathrm{H}$ CP-ENDOR spectroscopy at $94 \mathrm{GHz} / 3.4 \mathrm{~T}$.

In Chapter 5, the experimental and theoretical CP-ENDOR approach is adapted and 
expanded to the spin system of an electron spin coupled to a deuterium. Firstly, for a solid understanding of the CP-process, the CP matching conditions and CP-ENDOR intensities are predicted analytically and numerically for a coupled two spin system $\left(\mathrm{e}^{-} /{ }^{2} \mathrm{H}\right)$. A numerical simulation routine which is suited to predict matching conditions and CP-ENDOR intensities is presented. Experiments on a deuterated malonic acid radical from a $\gamma$-irradiated single crystal are performed to validate the predictions.

In Chapter 6, a modification of the CP-ENDOR sequence is introduced. The so-called without preparation pulse (WOP) CP-ENDOR sequence provides enhanced sensitivity for the detection of small hyperfine couplings. The performance of the sequence is explored on a well-known EPR standard sample: the perdeuterated BDPA radical dispersed in a protonated matrix of polystyrene. This is a valuable reference sample to investigate the performance of the WOP CP-ENDOR sequence for samples containing several molecules of random orientation. In addition to the experiments, the excitation profile generated by the CP-pulses during the CP-ENDOR sequence is given using numerical and analytical simulations.

Finally, the WOP CP-ENDOR sequence is applied to the spin-trapped radical intermediate $\mathrm{ND}_{2} \mathrm{Y}_{731}$ in E. coli RNR for two purposes (Chapter 7). Firstly, the performance of the WOP CP-ENDOR sequence is compared to the well-established ENDOR sequences using a biological sample. Secondly, the WOP CP-ENDOR sequence is used to validate the structure of the $\mathrm{ND}_{2}$ functional group from orientation-selective WOP CP-ENDOR experiments in conjunction with simulations based on DFT predicted hyperfine and quadrupole tensor parameters. There, spectroscopic limitations of the standard ENDOR sequences prevented an unambiguous structural elucidation. 


\section{Materials and methods}

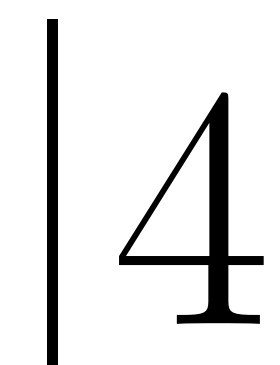

\subsection{Sample preparation}

\subsubsection{Deuterated malonic acid radical of a $\gamma$-irradiated single crystal}

The $\gamma$-irradiated single crystal of deuerated malonic acid radical was donated by Prof. Gunnar Jeschke (Dep. of Chemistry and Applied Bioscience, ETH Zürich), grown several years ago. According to the general procedure, the single crystals were grown from a saturated solution of deuterated malonic acid (98 atom\% D, $99 \%$ purity, purchased from Sigma Aldrich) in $\mathrm{D}_{2} \mathrm{O}$ (99.9 atom\% D, Sigma Aldich) by slow evaporation of the water. The radical was obtained after $\gamma$-irradiation with a ${ }^{60} \mathrm{Co}$ source. The crystal was sliced to fill a quartz W-band sample tube with ID/OD $=0.5 / 0.9 \mathrm{~mm}$ (Wilmad) to a height of $\sim 0.2 \mathrm{~cm}$. The capillary was sealed at both ends. After sample insertion into the magnet, the crystal orientation was identified by EPR and ENDOR measurements. The sample holder was then rotated in 20 degree increments and ESE-detected field sweeps and ENDOR spectra recorded at each position to identify the current hyperfine/quadrupole coupling (Literature values 88 : $A_{x} / 2 \pi=-4.4 \mathrm{MHz}, A_{y} / 2 \pi=-8.8 \mathrm{MHz}, A_{z} / 2 \pi=-14$ $\mathrm{MHz}$ and $\left.Q_{x} / 2 \pi=0.07 \mathrm{MHz}, Q_{y} / 2 \pi=-0.03 \mathrm{MHz}, Q_{z} / 2 \pi=-0.04 \mathrm{MHz}\right)$. Stepwise rotation and EPR control measurements showed hfc values ranging from $A / 2 \pi \sim \mid 4.5$ $8.9 \mid \mathrm{MHz}$. Accordingly, the two hfc tensor principle axis orientations $A_{x}$ and $A_{y}$ were examined perpendicular to the external magnetic field and $A_{z}$ parallel to the external magnetic field. Thus, the tensor principle axis orientation $A_{z}$ was assigned as the rotation axis during this procedure. The crystal orientation for the eNCP/CP-ENDOR experiments was chosen to result in a hfc value similar to the isotropic hfc $a_{\text {iso }}=9 \mathrm{MHz}$. 
This case represents best the two-spin system considered in the analytical and numerical treatment as will be discussed later.

\subsubsection{Protonated BDPA radical/ Perdeuterated BDPA radical}

Perdeuterated $\alpha, \gamma$-bisdiphenylene- $\beta$-phenylallyl (BDPA) was synthesized by the Facility for Synthetic Chemistry of the Max Planck Institute for biophysical Chemistry in Göttingen in 2012. The five step synthesis was performed in analogy to procedures published for the synthesis of protonated BDPA 29, , 90], but using fully deuterated educts and deuterated solvents. In the last step of the synthesis, a one-electron oxidation of the stable carbanion intermediate generates the BDPA radical. Protonated BDPA radical was purchased from Sigma Aldrich as BDPA complex with benzene (1:1). Polystyrene of average molecular weight 35000 was purchased from Sigma Aldrich. The following protocol was conducted by Brigitta Angerstein (RG Electron Spin Resonance, MPI for biophysical Chemistry Göttingen) in order to obtain equal dispersion of BDPA radicals in the (protonated) polystyrene matrix $(0.1 \% \mathrm{w} / \mathrm{w})$. Polystyrene and the respective BDPA radical were dissolved in $\mathrm{CHCl}_{3}$, then solvents were removed under nitrogen gas steam followed by lyophilization. The solid mixture of the BDPA radical in polystyrene matrix was finely ground, filled into a W-band sample tube (ID/OD $=0.5 / 0.9 \mathrm{~mm}$, Wilmad) to a height of $\sim 0.4 \mathrm{~cm}$ and sealed at both ends. The BDPA radical provides a valuable model radical to the EPR community since it is an air-stable, carbon-centered radical with high electron spin delocalization. The unpaired electron is predominantly located at the 1- and 3-positions of the compound's allyl core. It is is further stabilized by delocalization throughout the two biphenyl ring systems 21$]$ where 16 proton/deuterium nuclear spins are coupled (see Fig. 6.2, A, green). Accordingly, the five nuclei at the phenyl rings (see Fig. 6.2, A, purple) are not involved in electron spin delocalization. Finally, only two different sets of hfcs are present due to the high symmetry of the radical 91$]$. Thus, it can be analyzed as a spin system with one electron spin- $1 / 2$ coupled to two nuclear spins $I_{1}$ and $I_{2}$. 


\subsubsection{Spin trapping of $\mathrm{ND}_{2} \mathrm{Y}_{731}{ }^{\circ}$ in $E$. coli $\mathrm{RNR}$}

$\mathrm{NH}_{2} \mathrm{Y}_{731}-\alpha_{2}$ and wt- $\beta 2$ were provided by Prof. JoAnne Stubbe's research group (Massachusetts Institute of Technology, Cambridge) following previously established preparation protocols $79,85,92 . \mathrm{NH}_{2} \mathrm{Y}_{731}-\alpha_{2}$ and wt- $\beta_{2}$ were mixed 1:1 to a final concentration of $160-180 \mu \mathrm{M}$ in $\mathrm{D}_{2} \mathrm{O}$ assay buffer (50 mM HEPES, 15 mM MgSO4, 1 mM EDTA, $\mathrm{pH}$ 7.6) as previously described 17, 79. After initiating the radical reaction by adding $6 \mathrm{mM}$ ATP and $2 \mathrm{mM} \mathrm{CDP}$ at $25^{\circ} \mathrm{C}$, the reaction was hand-quenched in a W-band samples tube (ID/OD =0.5/0.9 mm, Wilmad) with assistance of Jun.-Prof. Müge Kasanmascheff (currently at Dept. of Physical Chemistry, TU Dortmund) in liquid $\mathrm{N}_{2}$ after $13 \mathrm{~s}$. The reference ESE field sweep spectrum of $\mathrm{Y}_{122}{ }^{\bullet}$ in the resting $\beta 2$ protein was subtracted from the $T=10 \mathrm{~K}$ ESE field sweep spectrum, yielding the $\mathrm{ND}_{2} \mathrm{Y}_{731}$ • spectrum obtained at $T=70 \mathrm{~K}$. After scaling the reference spectrum of $\mathrm{Y}_{122}{ }^{\bullet}$ at the low field side, where no intensity of $\mathrm{ND}_{2} \mathrm{Y}_{731} \bullet$ is expected, the $\mathrm{ND}_{2} \mathrm{Y}_{731}$ radical yield was determined to be $33 \%$.

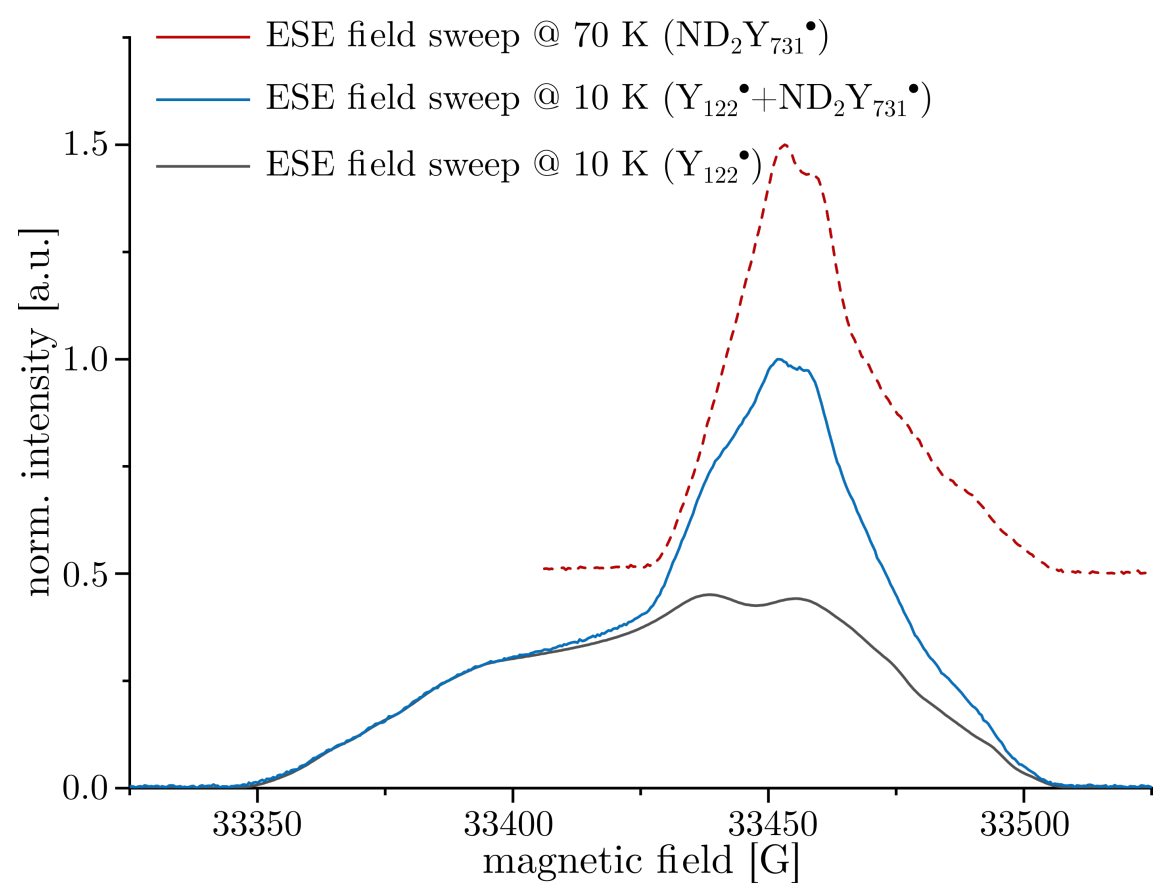

Figure 4.1: ESE-detected field sweep spectrum of $\mathrm{ND}_{2} \mathrm{Y}_{731}$ for radical yield determination. ESE field sweep spectra of $\mathrm{ND}_{2} \mathrm{Y}_{731}{ }^{\bullet}$ at $T=70 \mathrm{~K}$ (dashed red), $\mathrm{ND}_{2} \mathrm{Y}_{731}{ }^{\bullet}+\mathrm{Y}_{122}{ }^{\bullet}$ at $T=10 \mathrm{~K}$ (blue) and reference $\mathrm{Y}_{122} \bullet$ at $T=10 \mathrm{~K}$. The radical yield is obtained to be $33 \%$ from scaling the reference spectrum of $\mathrm{Y}_{122}{ }^{\bullet}$ at the low field side to the $\mathrm{ND}_{2} \mathrm{Y}_{731}{ }^{\bullet}+\mathrm{Y}_{122}{ }^{\bullet}$ spectrum. Exp. conditions: $T=70 \mathrm{~K}$, $t_{\pi / 2}=20 \mathrm{~ns}, \tau=240 \mathrm{~ns}$, shot repetition time $=3 \mathrm{~ms}, 50$ shot $/$ point, 10 scans. $T=10 \mathrm{~K}, t_{\pi / 2}=24 \mathrm{~ns}, \tau=240 \mathrm{~ns}$, shot repetition time $=150 \mathrm{~ms}, 5 \mathrm{shot} /$ point, 6 scans. Reference $\mathrm{Y}_{122}{ }^{\bullet}$ spectrum recorded by Dr. Tomislav Argirević during his $\mathrm{PhD}$ project at our research group. $T=10 \mathrm{~K}, t_{\pi / 2}=20 \mathrm{~ns}, \tau=340 \mathrm{~ns}$, shot repetition time $=40 \mathrm{~ms}$, shots $/$ point $=20$, scans $=125$. 


\subsection{Experimental setup}

Experiments were performed on a Bruker Elexsys E680 spectrometer operating at $\nu_{\mathrm{EPR}}=94 \mathrm{GHz}$ (W-band). The instrument revealed $400 \mathrm{~mW}$ output power (Power upgrade 2) which corresponds to our $\mathrm{TE}_{011}$ cavity achieving microwave fields of up to $\omega_{1 \mathrm{e}} / 2 \pi \approx 10 \mathrm{MHz}$. Typical $\pi$ inversion pulses at maximum power where $\mathrm{t}_{\pi, \mathrm{mw}}=40$ $50 \mathrm{~ns}$ as determined from Rabi nutation. The W-band bridge was upgraded by the manufacturer to $2 \mathrm{~W}$ output power. After the upgrade microwave fields of up to $\omega_{1 \mathrm{e}} / 2 \pi \approx 40 \mathrm{MHz}$ where achievable for our $\mathrm{TE}_{011}$ cavity resulting in an inversion pulse length of $t_{\pi, \mathrm{mw}}=16-20 \mathrm{~ns}$. A commercial Bruker ENDOR probehead (Bruker TeraFlex) fed with rf power of about 200 Watt (Amplifier research) results in ${ }^{2} \mathrm{H}$ and ${ }^{1} \mathrm{H}$ rf inversion pulses of $\mathrm{t}_{\pi, \mathrm{rf}}=25-38 \mu$ s obtained from Rabi nutations on the respective sample (see Fig. 4.3). The nuclear Rabi nutations were performed at the most intense hf line in the ENDOR spectrum of each sample. The ENDOR spectra displayed in this thesis are shown centered at the ${ }^{2} \mathrm{H} /{ }^{1} \mathrm{H}$ Larmor frequencies at $3.4 \mathrm{~T}$ of $\nu_{\mathrm{n}}^{2 \mathrm{H}} \approx 22 \mathrm{MHz}$ and $\nu_{\mathrm{n}}^{1 \mathrm{H}} \approx 142 \mathrm{MHz}$, respectively. Detailed experimental parameters are listed in figure captions.

\subsubsection{ESE-detected field sweep experiments}

The electron-spin echo (ESE) detected field sweep spectra were recorded with the Hahnecho pulse sequence $t_{\pi / 2}-\tau-t_{\pi}$ illustrated in Fig.4.2. Pulse durations of $t_{\pi} \approx 40-50 \mathrm{~ns}$ and a separation of $\tau=200-300 \mathrm{~ns}$ between the two pulses were used.

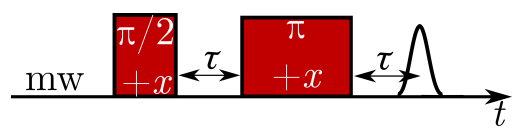

Figure 4.2: Pulse timing and pulse phases in Hahne-echo experiments. A $\pi / 2$-pulse of $t_{\pi, \mathrm{mw}}=1 /\left(4\left(\omega_{1 \mathrm{e}} / 2 \pi\right)\right)$ and a $\pi$-pulse of $t_{\pi, \mathrm{mw}}=1 /\left(2\left(\omega_{1 \mathrm{e}} / 2 \pi\right)\right)$ separated by the free evolution time $\tau$ are applied with $+x$ phases in order to obtain the Hahn Echo at the timepoint $2 \tau$ after the first pulse.

\subsubsection{Rabi-nutations}

The mw and rf pulse durations $t_{\mathrm{t}, \mathrm{mw}}, t_{\mathrm{t}, \mathrm{rf}}$ and corresponding field strength $\omega_{1 \mathrm{e}} / 2 \pi, \omega_{1 \mathrm{n}} / 2 \pi$ were determined by electron and nuclear spin Rabi nutations for each experiment as 
shown in Fig. 4.3. The nuclear Rabi nutations were performed at the most intense hf line in the ENDOR spectrum of each sample.

A) mw-nutation sequence

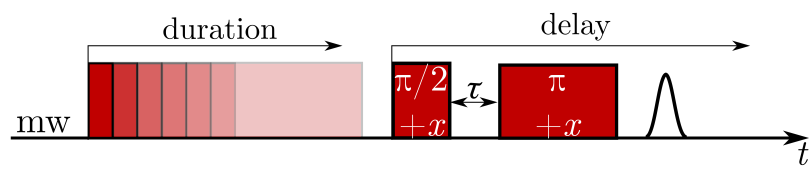

C) rf-nutation experiments

B) rf-nutation sequence
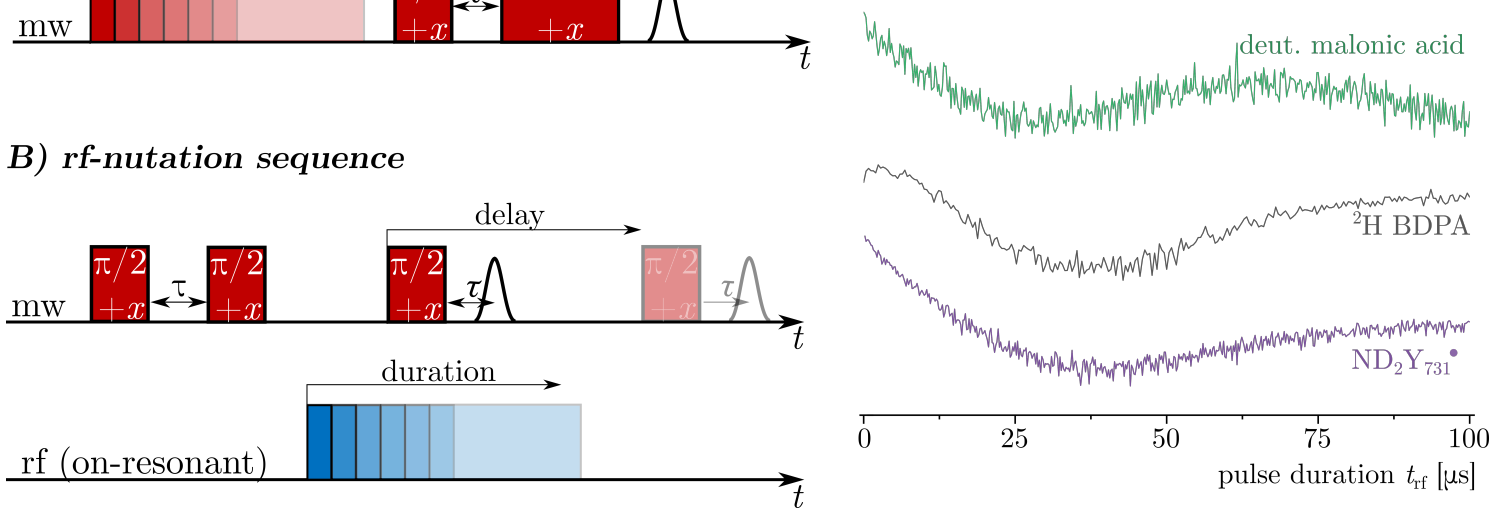

Figure 4.3: Electron spin and nuclear spin Rabi-nutation sequences. A) Schematic illustration of the mw-nutation sequence. B) Schematic illustration of the rf-nutation sequence. $C$ ) rf-nutation experiments on the deuterated malonic acid single crystal radical (green), ${ }^{2} \mathrm{H}-\mathrm{BDPA}$ (grey) and $\mathrm{ND}_{2} \mathrm{Y}_{731} \bullet$ (purple). Exp. condtitions: Malonic acid: $\nu_{\mathrm{EPR}}=94 \mathrm{GHz}, T=40 \mathrm{~K}, t_{\pi / 2, \mathrm{mw}}=20 \mathrm{~ns}, \tau=200 \mathrm{~ns}$, $\nu_{\mathrm{rf}}=25.9 \mathrm{MHz}$, shot repetition time $=100 \mathrm{~ms}, 1$ shot $/$ point, $\Delta t_{\mathrm{rf}}=200 \mathrm{~ns}, x-$ axis $=1024$ points, 6 scans; ${ }^{2} H-B D P A: \nu_{\mathrm{EPR}}=94 \mathrm{GHz}, T=80 \mathrm{~K}, t_{\pi / 2, m w}=24 \mathrm{~ns}$, $\tau=200 \mathrm{~ns}, \nu_{\mathrm{rf}}=21.3 \mathrm{MHz}$, shot repetition time $=15 \mathrm{~ms}, 1 \mathrm{shot} /$ point, $\Delta t_{\mathrm{rf}}=400 \mathrm{~ns}$, $x$-axis $=256$ points, 25 scans; $N D_{2} Y_{731}{ }^{\bullet}: \nu_{\mathrm{EPR}}=94 \mathrm{GHz}, T=10 \mathrm{~K}, t_{\pi / 2, \mathrm{rf}}=24 \mathrm{~ns}$, $\tau=200 \mathrm{~ns}, \nu_{\mathrm{rf}}=21.1 \mathrm{MHz}$, shot repetition time $=100 \mathrm{~ms}, 1 \mathrm{shot} /$ point, $\Delta t_{\mathrm{rf}}=200 \mathrm{~ns}$, $x$-axis $=512$ points, 5 scans.

\subsection{3. eNCP experiments}

The $+x$ and $+y$ mw channel power were attenuated to match the $\omega_{1 \mathrm{e}} / 2 \pi$ given in the respective figure captions of the experiments. The applied mw power was calculated from the Rabi frequencies determined by electron-spin Rabi-nutations. The primary criterion for the listed $\omega_{1 \mathrm{e}} / 2 \pi$ values was $A / 2 \pi>\omega_{1 \mathrm{e}} / 2 \pi$ as reported in 25 . The described pulse timings and phases are illustrated in Fig. 4.4. A $\pi / 2$ preparation $+x$ pulse of duration $t_{\pi / 2, \mathrm{mw}}=1 /\left(4\left(\omega_{1 \mathrm{e}} / 2 \pi\right)\right)$ followed by a mw spinlock pulse $t_{\mathrm{CP}}=150-200 \mu \mathrm{s}$ with $90^{\circ}$ phase shift $(+y$ microwave channel) were set. The CP pulse length was optimized to obtain maximum loss of electron spin polarization in the eNCP spectrum. In order to keep the duty cycle $\leq 1 \%$ and avoid heating effects, a maximum rf length during spinlock was set to $200 \mu \mathrm{s}$. The rf pulse during spinlock was set simultaneous to the microwave pulse with a stochastically swept rf frequency. A $\pi$-pulse of $t_{\pi, \mathrm{mw}}=1 /\left(2\left(\omega_{1 \mathrm{e}} / 2 \pi\right)\right)$ with a delay time $t_{\mathrm{d}}$ of $\approx 1 \mu \mathrm{s}$ after the spinlock pulse was used to monitor the electron spin 
polarization loss as a function of the rf frequency. The experiment was performed with selective excitation (= soft mw pulses) and detection of one EPR transition.

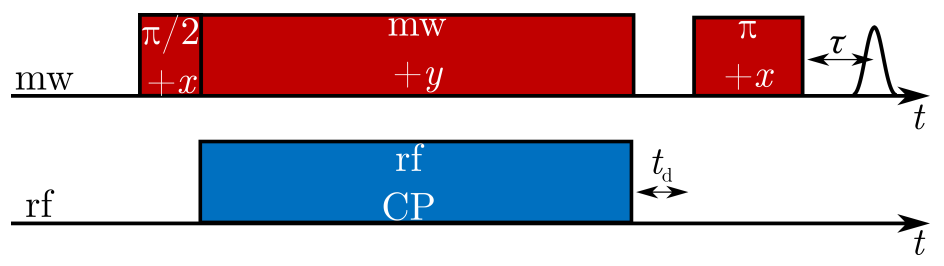

Figure 4.4: Pulse timing and pulse phases in eNCP experiments. The eNCP sequence consists of a $\pi / 2 \mathrm{mw}$ pulse with $+x$ phase followed by a $90^{\circ}$ phase shifted mw pulse with $+y$ phase. Concomitantly to the mw pulse, an rf pulse with stochastically swept $\mathrm{rf}$ frequency is applied. After another delay time $t_{\mathrm{d}}$ of $\approx 1 \mu \mathrm{s}$ a $\pi / 2$ pulse with $+x$ phase is used to monitor the electron spin polarization as a function of the rf frequency.

\subsubsection{ENDOR experiments}

\subsubsection{CP-ENDOR}

Fig. 4.5 shows the schematic CP-ENDOR sequence with pulse timings and phases as described in the following.

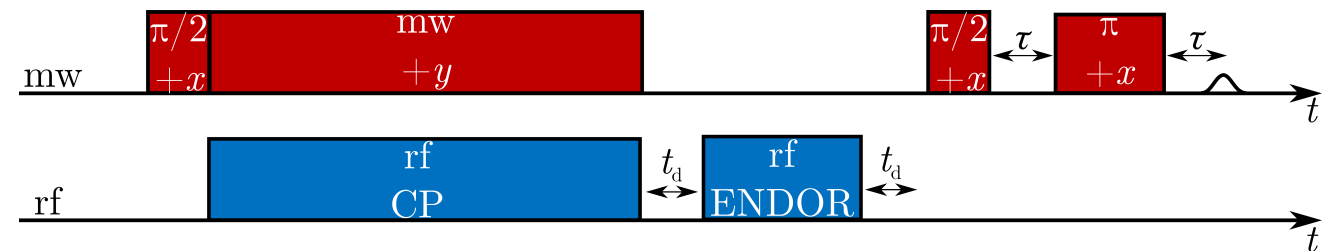

Figure 4.5: Pulse timing and pulse phases in CP-ENDOR experiments. The CP-ENDOR sequence consists of a $\pi / 2 \mathrm{mw}$ pulse with $+x$ phase followed by a $90^{\circ}$ phase shifted mw pulse with $+y$ phase. Concomitant to the mw pulse, an rf pulse with stochastically swept $r$ frequency is applied. After a delay time $t_{\mathrm{d}}$ of $1 \mu \mathrm{s}$, the frequency swept rf ENDOR pulse is applied. The ENDOR information was monitored by a read-out Hahn-echo sequence of $t_{\pi / 2}-\tau-t_{\pi}$ with on the mw $+x$ channel, set after a delay time of $t_{\mathrm{d}} \approx 1 \mu \mathrm{s}$ after the ENDOR rf pulse.

The power of the $+x$ and $+y$ mw channel was attenuated to match the $\omega_{1 \mathrm{e}} / 2 \pi$ as given in the figure captions of the respective experiments. The power was manually optimized for each experiment to obtain a reasonable compromise between hole width and $\mathrm{S} / \mathrm{N}$ ratio (excitation bandwidth) of the resulting CP-ENDOR spectrum. Firstly, a $\pi / 2$ preparation $+x$ pulse of duration $t_{\pi / 2, \mathrm{mw}}=1 /\left(4\left(\omega_{1 \mathrm{e}} / 2 \pi\right)\right)$ was set for standard CP-ENDOR. This pulse was skipped in WOP CP-ENDOR. The mw spinlock pulse of $t_{\mathrm{CP}}=150-200 \mu \mathrm{s}$ with $90^{\circ}$ phase shift was set on the $+y$ microwave channel. The pulse 
length was set according to the optimized value obtained from the eNCP experiment. In order to keep the duty cycle $\leq 1 \%$ and avoid heating effects a maximum rf length during spinlock was set to $200 \mu \mathrm{s}$. The rf pulse during spinlock was set concomitant to the microwave pulse with fixed rf. The rf was changed manually after a fixed number of averages or by a home-written PulseSPEL routine printed in Appendix F. Another rf pulse of $t_{\pi, \mathrm{rf}}=25-38 \mu \mathrm{s}$ on the same rf channel was set with a delay of $\approx 1 \mu \mathrm{s}$ after the spinlock pulse. The frequency of this pulse was swept over the ENDOR frequency range in a stochastic manner. A selective read-out Hahn-echo sequence of $t_{\pi / 2}-\tau-t_{\pi}$ with $t_{\pi / 2, \mathrm{mw}}=1 /\left(4\left(\omega_{1 \mathrm{e}} / 2 \pi\right)\right)$ and $\tau=800-1600$ ns on the microwave $+x$ channel was set after a delay time $t_{\mathrm{d}}$ of $\approx 1 \mu$ s after the ENDOR rf pulse. Longer mw pulses lead to a broader echo and thus longer $\tau$ values are required to sufficiently separate the echo from the FID and the spectrometer dead-time. Long $\tau$ evolution times, however, facilitate longer time duration in which relaxation occurs. Consequently, part of the magnetization has decayed before the detection. Thus, $\tau$ has to be optimized to the sample system intrinsic $T_{2}$ relaxation time at the given experimental conditions (magnetic field and temperature). Note, that the integrated CP-ENDOR echo intensity is significantly smaller compared to Davies and Mims ENDOR. Here, the CP-ENDOR effect is obtained as a gain in echo intensity. In CP-ENDOR, the electron spin polarization is transferred to the coupled nuclear spins in the CP step, thus minimal echo intensity is detected if the subsequent rf ENDOR pulse is off-resonant. When the rf ENDOR pulse is on-resonant with an NMR transition, the polarization is transferred back to the electron spin and and the electron-spin echo intensity is re-obtained. In contrast, the ENDOR effect in Davies and Mims ENDOR is obtained as a loss of electron-spin echo intensity. There, the intensity of the monitored EPR transition vanishes when the rf ENDOR pulse transfers polarization, in this case from the electron spin to the nucleus (for details see Subsec. 2.4.2 and Fig. 2.14).

\subsubsection{Davies ENDOR}

The $+\left\langle x>\right.$-channel mw power was adjusted to satisfy a selective $t_{\pi, \mathrm{mw}}=1 /\left(2\left(\omega_{1 \mathrm{e}} / 2 \pi\right)\right)$ preparation pulse with phase $+x$ (for sequence description see Sec. 2.3.2). The inversion pulse length at a particular mw attenuation was determined from a Rabi-nutation. The rf $\pi$-pulse of $25-38 \mu$ s was set with a delay of $\approx 1 \mu$ s after the preparation pulse. A read-out sequence of $t_{\pi / 2}-\tau-t_{\pi}$ on the microwave $+x$ channel with maximum mw power and phase $+x$ was set after a delay time $t_{\mathrm{d}}$ of $\approx 1 \mu$ s after the ENDOR rf pulse 
as shown in Fig. 4.6. The pulses were non-selective (hard pulses) in order to increase the detected echo intensity. Typical pulse lengths with $400 \mathrm{~mW}$ output power were $t_{\pi / 2}=24-30 \mathrm{~ns}$.

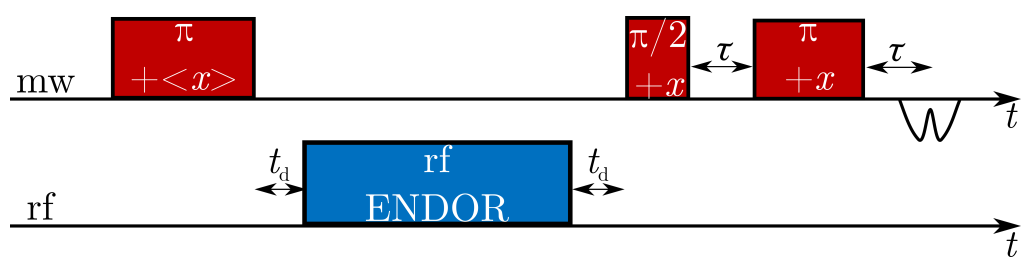

Figure 4.6: Pulse timing and pulse phases in Davies ENDOR experiments. The sequence consists of the following steps: a selective $\pi$ mw pulse with phase $+x$, time delay of $t_{\mathrm{d}}$, frequency-swept rf ENDOR inversion $\pi$ pulse, time delay of $t_{\mathrm{d}}$ and Hahn Echo read out with non-selective pulses. All mw phases are applied with $+x$ phase.

\subsubsection{Mims ENDOR}

Mims ENDOR was performed with the maximum available mw output power before the upgrade, resulting in pulses of $t_{\pi / 2}=24 \mathrm{~ns}$ duration. Two mw $\pi / 2$ pulses separated by $\tau$ were applied on the $+x$ channel. The ENDOR rf $\pi$-pulse of $25-38 \mu$ s was set with a delay of $\approx 1 \mu$ s before and after the pulse. Another $\pi / 2$ pulse on the $+x$ channel with $+x$ phase was used to read-out the ENDOR information. Pulse timings and phases are illustrated in Fig. 4.7 .

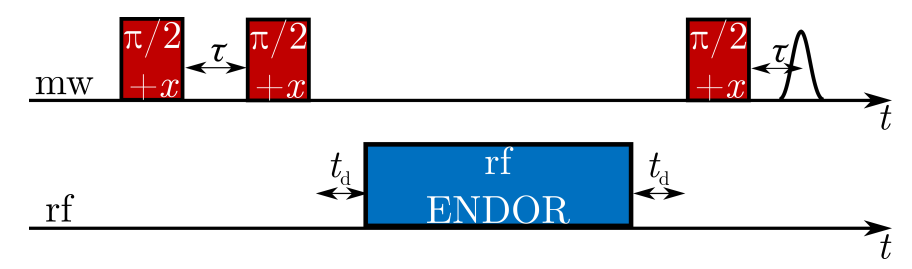

Figure 4.7: Pulse timing and pulse phases in Mims ENDOR experiments. Two non-selective $\pi / 2 \mathrm{mw}$ pulses separated by $\tau$ are applied with phases $+x$. This preparation step is followed by a time delay of $t_{\mathrm{d}}$, the frequency swept $\mathrm{rf}$ ENDOR inversion pulse and another time delay $t_{\mathrm{d}}$. Finally, a non-selective $\pi / 2$ pulse with phase $+x$ generates the monitored stimulated echo after $\tau$ evolution time.

\subsection{Numerical simulations}

The presented simulation routine based on density operator formalism was developed in collaboration with Dr. Robert Zeier and Prof. Steffen Glaser (Department of Chemistry, TU Munich). Firstly, a formalism for numerical prediction eNCP and CP-ENDOR spectra of a two-spin system with an electron spin $S=1 / 2$ coupled to a nuclear spin 
$I=1 / 2$ (not shown) was developed. This formalism was adapted and modified in order to describe the case of an electron spin $S=1 / 2$ coupled to a nuclear spin $I=1$ as presented in Section 4.3.1. Further, up to three coupled $I=1$ nuclear spins and powder averaging (Section 4.3.2.1), as well as orientation-selection (Section 4.3.3) on the basis of a simulation strategy developed previously in our research group were implemented 13 .

\subsubsection{Single crystal simulations}

The Hamiltonian $\mathcal{H}_{0}$ of the experimental system in the doubly rotating frame is defined as

$$
\mathcal{H}_{0}=\Delta \omega_{\mathrm{e}} S_{z}+\Delta \omega_{\mathrm{n}} I_{z}+A S_{z} I_{z}+Q\left(\frac{3}{2} I_{z}^{2}-\mathbb{1}\right)
$$

where $A / 2 \pi$ and $Q / 2 \pi$ denote hyperfine and quadrupole interaction strength, and $\omega_{\mathrm{e}}$ and $\omega_{\mathrm{n}}$ are the Larmor frequencies of electron (e) and nuclear (e) spin. For simplification of the mathematical treatment, we consider HFA for both spins, thus, terms containing $I_{x} / S_{x}$ and $I_{y} / S_{y}$ are neglected (pseudo-secular hf terms $B_{x}$ and $B_{y}$ ). This is a valid assumption since previous published CP-ENDOR studies $25[27]$ demonstrated that the CP-mechanism does not rely on these terms. Furthermore, approaches in which these terms are considered have been taken, however, numerical and analytical calculations resulted unenforceable due to the resulting complexity of the Hamiltonian.

At next, control functions $u_{x}(t), u_{y}(t), v_{x}(t)$ and $v_{y}(t)$ are added to the Hamiltonian given in Eq. 4.1. They detail how the Hamiltonian changes during a time evolution when applying pulses of $S_{x}, S_{y}, I_{x}$ and $I_{y}$. This results in the Hamiltonian

$$
\mathcal{H}(t)=\mathcal{H}_{0}+2 \pi u_{x}(t) S_{x}+2 \pi u_{y}(t) S_{y}+2 \pi v_{x}(t) I_{x}+2 \pi v_{y}(t) I_{y}
$$

The Larmor frequencies of the electron and the nuclear spin are given by $\omega_{e}$ and $\omega_{n}$. The following description is given in the doubly rotating frame corresponding to the electron and the nuclear spin carrier frequencies $\Delta \omega_{\mathrm{e}}=\omega_{\mathrm{e}}-\omega_{\mathrm{mw}}$ and $\Delta \omega_{\mathrm{n}}=\omega_{\mathrm{n}}-\omega_{\mathrm{rf}}$ and applying the rotating wave approximation. With the latter, all terms oscillating with $2 \omega_{\mathrm{mw}}$ are neglected, assuming that they are not actively influencing the rotating frame.

The general numerical simulation strategy critically relies on the choice of the rotating frame. The chosen rotating frame allows to treat almost all Hamiltonians $\mathcal{H}_{m}$ which act during the time steps of the eNCP and CP-ENDOR sequence as time independent. 
Accordingly, the control functions $u_{x}(t), \ldots, v_{y}(t)$ become time independent $u_{x}, \ldots, v_{y}$. Only the Hamiltonian $\mathcal{H}_{\text {ENDOR }}(t)$ for the ENDOR step is treated as not constant. This is required since during the CP-ENDOR sequence two rf pulses (CP and ENDOR) with different rf carrier frequencies $\left(\omega_{\mathrm{rf}}^{\mathrm{CP}}\right.$ and $\left.\omega_{\mathrm{rf}}^{\mathrm{ENDOR}}\right)$ are applied. Each of the steps can be described in its own nuclear spin rotating frame of either $\Delta \omega_{\mathrm{n}}=\omega_{\mathrm{n}}-\omega_{\mathrm{rf}}^{\mathrm{CP}}=\Delta \omega_{\mathrm{n}}^{\mathrm{CP}}$ or $\Delta \omega_{\mathrm{n}}=\omega_{\mathrm{n}}-\omega_{\mathrm{rf}}^{\mathrm{ENDOR}}=\Delta \omega_{\mathrm{n}}^{\mathrm{ENDOR}}$, however they are not identical $\left(\Delta \omega_{\mathrm{n}}^{\mathrm{ENDOR}} \neq \Delta \omega_{\mathrm{n}}^{\mathrm{CP}}\right)$. Thus, once the nuclear spin rotating frame is defined to be identical to the CP rf, the ENDOR step becomes time-dependent. In the following, first the general strategy for time-independent Hamiltonians as introduced in Subsec. 4.3.1.3 is presented and later the more involved ENDOR step is discussed. In a time step of duration $t_{i}$, the density matrix $\rho$ is mapped to $U_{m}\left(t_{i}\right) \rho U_{m}\left(t_{i}\right)^{-1}$. The unitary matrix $U_{m}=\exp \left(-\mathrm{i} H_{m} t_{i}\right)$ is computed by numerical matrix exponentiation (see Section 2.2). The resulting density matrix $U_{m}\left(t_{i}\right) \rho U_{m}\left(t_{i}\right)^{-1}$ is calculated by two numerical matrix multiplications.

\subsubsection{1. eNCP simulation}

The eNCP simulation was performed with a sequence of five simulation steps while varying the $\mathrm{CP}$ rf $\left(\omega_{\mathrm{rf}}^{\mathrm{CP}}\right)$, illustrated in Fig. 4.8 .

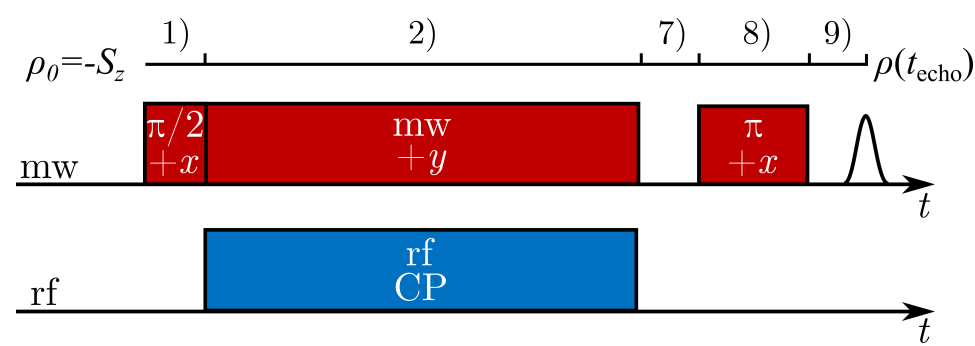

Figure 4.8: Illustration of the eNCP simulation steps. Five steps are considered: 1) $\pi / 2$ preparation pulse selectively on one EPR transition with phase $+x, 2$ ) long mw irradiation pulse with phase $+x$ concomitantly to the frequency swept $\mathrm{rf}$ pulse, 7) free evolution, 8) mw $\pi$ pulse of phase $+x, 9)$ free evolution. The initial state for all simulations is $\rho_{0}=-S_{z}$ which evolves to $\rho\left(t_{\text {echo }}\right)$ at the echo detection. Note : Numbering of steps was adapted to match with Fig. 4.9. 
The five steps (listed below) will be described in the following. Precise parameters can be found in the figure captions.

$$
\begin{aligned}
\text { 1) } \mathcal{H}_{1} & :=\mathcal{H}_{0}+\omega_{1 \mathrm{e}} S_{x} \\
\text { 2) } \mathcal{H}_{2} & :=\mathcal{H}_{\mathrm{CP}}:=\mathcal{H}_{0}+\omega_{1 \mathrm{e}} S_{y}+\omega_{1 \mathrm{n}} I_{x} \\
\text { 7) } \mathcal{H}_{7} & :=\mathcal{H}_{0} \\
\text { 8) } \mathcal{H}_{8} & :=\mathcal{H}_{1}:=\mathcal{H}_{0}+\omega_{1 \mathrm{e}} S_{x} \\
\text { 9) } \mathcal{H}_{9} & :=\mathcal{H}_{0}
\end{aligned}
$$

The eNCP sequence starts from the density matrix $\rho_{0}=-S_{z}$. In the first step, a $\pi / 2$ pulse on the electron spin is applied along the $+x$-direction. This corresponds to a time evolution with respect to the time-independent Hamiltonian $\mathcal{H}_{1}$ with $\omega_{1 \mathrm{e}} / 2 \pi$ and the duration $t_{1}$. The second step is the CP step where mw and rf irradiation are applied concomitantly along the $+y$-direction. In particular the time-independent Hamiltonian $\mathcal{H}_{2}$ with $\omega_{1 \mathrm{n}} / 2 \pi$ and $\omega_{1 \mathrm{e}} / 2 \pi$ is applied for $t_{2}$. Note that the CP rf-offset $\Delta \omega_{\mathrm{n}}^{\mathrm{CP}}$ is implicitly contained in $\mathcal{H}_{0}$. The third step (7) consists of a free evolution with respect to $\mathcal{H}_{7}$ which lasts $t_{7}$. In the fourth step (8), a $\pi$ pulse on the electron spin is applied along the $+x$-direction. This pulse is modeled by the time-independent Hamiltonian $\mathcal{H}_{8}$ which is applied for $t_{8}$. The fifth step (9) is another free evolution step with respect to $\mathcal{H}_{7}$ which lasts $t_{7}$. The eNCP signal is obtained from $-<S_{y}\left(t_{\text {echo }}\right)>$ after the fifth step. The following parameters have to be optimized for each of the Hamiltonians accordingly: (a) electron spin offset $\Delta \omega_{\mathrm{e}}(= \pm A, 0)$, (b) hyperfine coupling $A / 2 \pi$, (c) quadrupole coupling $Q / 2 \pi$, (d) microwave frequency amplitude $\omega_{1 \mathrm{e}} / 2 \pi$, (e) radio frequency amplitude $\omega_{1 \mathrm{n}} / 2 \pi$, and (f) time intervals $t_{i}$ for each propagator. 


\subsubsection{CP-ENDOR simulation}

The CP-ENDOR simulation consists of 9 steps as illustrated in Fig. 4.9 and listed in the following, where CP- and ENDOR frequency are varied:

$$
\begin{aligned}
& \text { 1) } \mathcal{H}_{1}:=\mathcal{H}_{0}+\omega_{1 \mathrm{e}} S_{x} \\
& \text { 2) } \mathcal{H}_{2}:=\mathcal{H}_{\mathrm{CP}}:=\mathcal{H}_{0}+\omega_{1 \mathrm{e}} S_{y}+\omega_{1 \mathrm{n}} I_{x} \\
& \text { 3) } \mathcal{H}_{3}:=\mathcal{H}_{0} \\
& \text { 4) } \mathcal{H}_{4}:=\mathcal{H}_{\mathrm{ENDOR}}(t):=\mathcal{H}_{0}+\omega_{1 \mathrm{n}} \cos \left(\Delta \omega_{\mathrm{rf}}^{\text {Diff }} t\right) I_{x}+\omega_{1 \mathrm{n}} \sin \left(\Delta \omega_{\mathrm{rf}}^{\text {Diff }} t\right) I_{y} \\
& \text { 5) } \mathcal{H}_{5}:=\mathcal{H}_{3}:=\mathcal{H}_{0} \\
& \text { 6) } \mathcal{H}_{6}:=\mathcal{H}_{1}:=\mathcal{H}_{0}+\omega_{1 \mathrm{e}} S_{x} \\
& \text { 7) } \mathcal{H}_{7}:=\mathcal{H}_{0} \\
& \text { 8) } \mathcal{H}_{8}:=\mathcal{H}_{0}+\omega_{1 \mathrm{e}} S_{x} \\
& \text { 9) } \mathcal{H}_{9}:=\mathcal{H}_{7}:=\mathcal{H}_{0}
\end{aligned}
$$

where $\Delta \omega_{\mathrm{rf}}^{\mathrm{Diff}}=\omega_{\mathrm{rf}}^{\mathrm{ENDOR}}-\omega_{\mathrm{rf}}^{\mathrm{CP}}$.

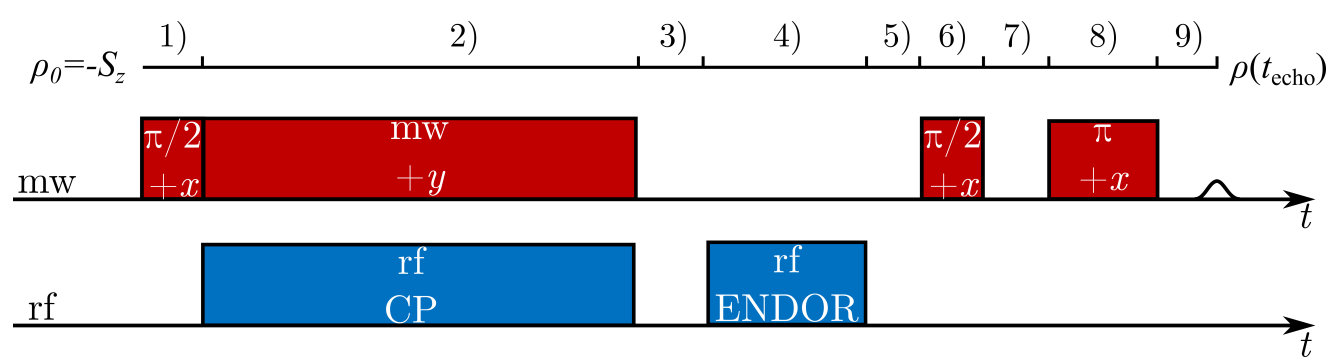

Figure 4.9: Illustration of the CP-ENDOR simulation steps. Nine steps are considered: 1) $\pi / 2$ preparation pulse selectively on one EPR transition with phase $+x, 2)$ long $\mathrm{mw}$ irradiation pulse with phase $+x$ concomitantly to the CP rf pulse, 3) free evolution, 4) rf $\pi$ ENDOR inversion pulse, 5) free evolution, 6) selective $\pi / 2$ mw pulse with phase $+x, 7)$ free evolution, 8 ) selective $\pi$ mw pulse with phase $+x$, 9 ) free evolution. The initial state for all simulations is $\rho_{0}=-S_{z}$ which evolves to $\rho\left(t_{\text {echo }}\right)$ at the echo detection.

The CP-ENDOR sequence starts from the density matrix $\rho_{0}=-S_{z}$. The first three steps are identical to the eNCP experiment. The fourth step is identified as the ENDOR step which applies an rf irradiation with incrementation of the rf frequency on the nuclear spin for $t_{4}$. The corresponding time-dependent Hamiltonian is given by $\mathcal{H}_{4}$. The fifth step is identical to the third step and the sixth step is identical to the first step. The seventh step consists of a free evolution with respect to $\mathcal{H}_{7}$ which lasts $t_{7}$. In the 
eighth step, a $\pi$-pulse on the electron spin is applied along the $+x$-direction. This pulse is modeled by the time-independent Hamiltonian $\mathcal{H}_{8}$ which is applied for $t_{8}$. The ninth step is identical to the seventh step. The echo intenstiy is obtained from $\left\langle S_{y}\left(t_{\text {echo }}\right)\right\rangle$ after the ninth step.

\subsubsection{Efficient simulation of the ENDOR step}

In the numerical simulation the CP-ENDOR sequence is described in a doubly-rotating frame. The rotating frame frequency for the nuclear spin $I$ is chosen to be identical to the $\mathrm{rf} \omega_{\mathrm{rf}}^{\mathrm{CP}}$ that is applied during the CP $\operatorname{step}\left(\mathcal{H}_{2}:=\mathcal{H}_{\mathrm{CP}}\right)$. Hence, in this rotating frame, the offset frequency is given by $\Delta \omega_{\mathrm{n}}=\omega_{\mathrm{n}}-\omega_{\mathrm{rf}}^{\mathrm{CP}}$. However, during the ENDOR period (step 4) the irradiation frequency $\omega_{\mathrm{rf}}^{\mathrm{ENDOR}}$ is generally different from $\omega_{\mathrm{rf}}^{\mathrm{CP}}$ which results in a time-dependent oscillating rf Hamiltonian in the rotating frame defined by $\omega_{\mathrm{rf}}^{\mathrm{CP}}$. In principle, the ENDOR step could be implemented by discretizing the ENDOR period in small time-steps during which the rf Hamiltonian is approximately constant, and, calculating the time evolution of the density operator for these time steps. However, this naïve approach would be computationally expensive because it requires the calculation of a large number of steps. For example, for a difference frequency $\Delta \omega_{\mathrm{rf}}^{\mathrm{Diff}}=\omega_{\mathrm{rf}}^{\mathrm{ENDOR}}-\omega_{\mathrm{rf}}^{\mathrm{CP}}$ of $10 \mathrm{MHz}$, one oscillation period corresponds to $T_{\mathrm{osc}}=1 / \Delta \omega_{\mathrm{rf}}=100 \mathrm{~ns}$, the time steps should be smaller than $T_{\text {osc }} / 10=10 \mathrm{~ns}$ in order to faithfully approach the modulation by a piecewise constant function. For a typical ENDOR period of $30 \mu \mathrm{s}$, this would require at least 3000 time steps to be calculated. A computationally much more efficient approach consists of two simple steps:(a) Running the calculation during the ENDOR period (step 4) in the rotating frame defined by the ENDOR nuclear rf irradiation $\omega_{\mathrm{rf}}^{\mathrm{ENDOR}}$ in which the rf Hamiltonian is constant and the offset frequency of the nuclear spin is $\Delta \omega_{\mathrm{n}}^{\text {ENDOR }}=\omega_{\mathrm{n}}-\omega_{\mathrm{rf}}^{\text {ENDOR }}$. Hence, the Hamiltonian $\mathcal{H}_{4}:=\mathcal{H}_{\text {ENDOR }}(t)$ can be reformulated to the constant term:

$$
\mathcal{H}_{4 a}=\Delta \omega_{\mathrm{e}} S_{z}+\Delta \omega_{\mathrm{n}}^{\mathrm{ENDOR}} I_{y}+A S_{z} I_{z}+\omega_{Q}\left(\frac{3}{2}\left(I_{z}\right)^{2}-1\right)+\omega_{1 \mathrm{n}} I_{x}
$$

This is followed by step (b) which takes into account the different rotating frequencies during the ENDOR step of duration $t_{4}$ by the density operator transformed by $U_{4 b}=$ $\exp \left(-\mathrm{i} H_{4 b} t_{4}\right)$ with

$$
\mathcal{H}_{4 b}=\left(\omega_{\mathrm{rf}}^{\mathrm{ENDOR}}-\omega_{\mathrm{rf}}^{\mathrm{CP}}\right) I_{z}=\Delta \omega_{\mathrm{rf}}^{\mathrm{Diff}} I_{z}
$$


In summary, the density matrix $\rho$ is mapped to the density matrix $U_{4 b}\left(U_{4 a} \rho U_{4 a}^{-1}\right) U_{4 b}^{-1}$ in the ENDOR step using the two sub-steps (a) and (b), which can both be modeled using a time-independent Hamiltonian. This provides an efficient approach to compute the effect of the ENDOR step.

\subsubsection{Heuristic simulation of the effect of $B_{1}$ inhomogeneity and relax- ation during the $\mathrm{CP}$ and free evolution step}

The effect of $B_{1}$ inhomogeneity and relaxation was taken into account in our simulations in consideration of the elaborations published in 25 . In principle, the $B_{1}$ inhomogeneity effect could be numerically simulated by averaging a range of $B_{1}$ amplitudes. This would, however, lead to a significant simulation overhead and a decrease in efficiency. Here, a heuristic simulation approach was used to model the effect of $B_{1}$ inhomogeneity and the relaxation accumulated during the second (i.e. CP) and third step, which are assumed to be the primary incoherent contribution during the CP-ENDOR sequence. After the application of the Hamiltonian $\mathcal{H}_{2}=\mathcal{H}_{\mathrm{CP}}$ during the second step, (a) the density matrix is transformed into the eigenbasis of $\mathcal{H}_{2}=\mathcal{H}_{\mathrm{CP}}$, (b) all non-diagonal entries of the density matrix are removed, and (c) the density matrix is transformed back to its original basis. Then, (d) the unitary $U_{3}$ is applied to the density matrix and (e) all of its non-diagonal entries are removed again. The new substeps (a) to (c) emulate the averaging effect of $B_{1}$ inhomogeneity during the CP step as well as a transverse relaxation. As before, sub-step (d) accounts for the free evolution during the third step. The substep (e) is considered to model the effect of fast transverse relaxation. This heuristic approach leads to only a minor computational overhead while capturing important experimental contributions during the CP-ENDOR sequence.

\subsubsection{Powder pattern simulations of ${ }^{2} \mathrm{H}-\mathrm{BDPA}$}

\subsubsection{Simulation strategy for powder pattern samples - ${ }^{2}$ H-BDPA simu- lations}

For powder pattern CP-ENDOR simulations of ${ }^{2} \mathrm{H}$-BDPA, the routine described in the previous section was extended to consider two more aspects: 1) a second coupled deuterium (according to the spin density distribution as discussed in Subsec. 4.1.2) and 2) $g$ - and $A$-ansiotropy effects. Orientation-selection is not taken into account at this point, because it is assumed to have a minor contribution to the obtained spectra ( $g$-tensor not 
resolved). The first aspect was implemented by straight-forward matrix expansion (see Appendix B.2). A coupled spin-system of $S=1 / 2, I_{1}=1, I_{2}=1$ results in a density matrix of dimension $18 \times 18$. Further, the $g / A$-anisotropy implementation requires two modifications: (a) starting from hfc tensor values in the hyperfine principle axis system (Table 6.1), its contribution with respect to the laboratory system is calculated. This is done for a powder average of $N_{\phi}$ and $N_{\theta}$ orientations in order to cover all possible orientations of the two hf tensors (see Subsec. 2.1.1.1 for definitions of $\theta$ and $\phi$ ). The calculation was implemented following a previously in-house developed protocol ${ }^{[13]}$. The obtained hf-values are handed over to the CP-ENDOR part of the simulation. Consequently, the absolute intensity obtained in the simulations is proportional to $N_{\phi, \theta}$. For ${ }^{2} \mathrm{H}$-BDPA simulation, $N_{\phi, \theta}=32$ was optimized manually by line shape analysis. Because larger $N_{\phi, \theta}$ values did not considerably change the obtained ENDOR line shape, a full powder pattern was observed at these numbers. Smaller values, however, indicated line shape distortions resulting from incomplete powder average.

(b) $g$-anisotropy is considered via summation of CP-ENDOR simulations at the electron spin offsets $\Delta \omega_{\mathrm{e}}=-A, 0,+A$ (on-resonant components) for the case of one coupled deuterium. Previously published studies 25 suggested that the $g$-anisotropy effect, which shifts EPR transitions to slightly different resonances, can be introduced to the CPENDOR simulation by averaging the results obtained for different spin systems at different $\Delta \omega_{\mathrm{e}}$. This can be visualized considering an idealized case of only three spin packets and $g$-anisotropy, which shifts resonances by $\pm A$ as illustrated in Fig. 4.10. In this particular case $\operatorname{EPR} \alpha, \beta$ and $\gamma$ of different spin packets (1,2 and 3$)$ share an excitation position in the EPR spectrum (grey box). In particular, EPR $\beta$ of spin packet 1, EPR $\alpha$ of spin packet 2 and EPR $\gamma$ of spin packet 3 share a resonance frequency (Fig. 4.10, grey box). They will be all excited at once by the excitation pulse. However, for a given rf offset their CP-ENDOR spectra are different because they do not necessarily share a CP matching condition (B). It is the sum of the three CP-ENDOR spectra (on $\alpha, \beta$ and $\gamma$ ) that predicts the CP-ENDOR spectrum at this resonance position and $\mathrm{rf}$ offset $(\mathrm{B}$, bottom). This idea is implemented by calculating the CP-ENDOR spectrum at each electron spin offset $\Delta \omega_{\mathrm{e}}=-A, 0,+A$ for every hfc $A$ contributing to the powder pattern $\left(N_{\phi, \theta}\right)$ and summing them up. The number of electron spin offset which have to be considered, however, scales with the number of coupled nuclear spins. The two hfcs considered for the BDPA powder simulation show $A_{1} \neq A_{2}$ for almost all orientations which results in nine EPR resonances per 
spin packet. This means nine electron spin offsets need to be considered for the above described extension.

Additional contributions from off-resonant components $\Delta \omega_{\mathrm{e}} \neq-A, 0,+A$ were neglected. Indeed, we assume that, due to the complex-multiple spin systems ( 9 and 27 electron spin offsets considered for BDPA and RNR simulations, respectively) in conjunction with their small (on the order of the electron spin excitation bandwidth) and similar hfc values (in the same order of magnitude), several off-resonant components are inherently considered according to the excitation bandwidth of the pulse.

\section{A) $\mathbf{E P R} \quad S=1 / 2, I=1$}

spin packet 1

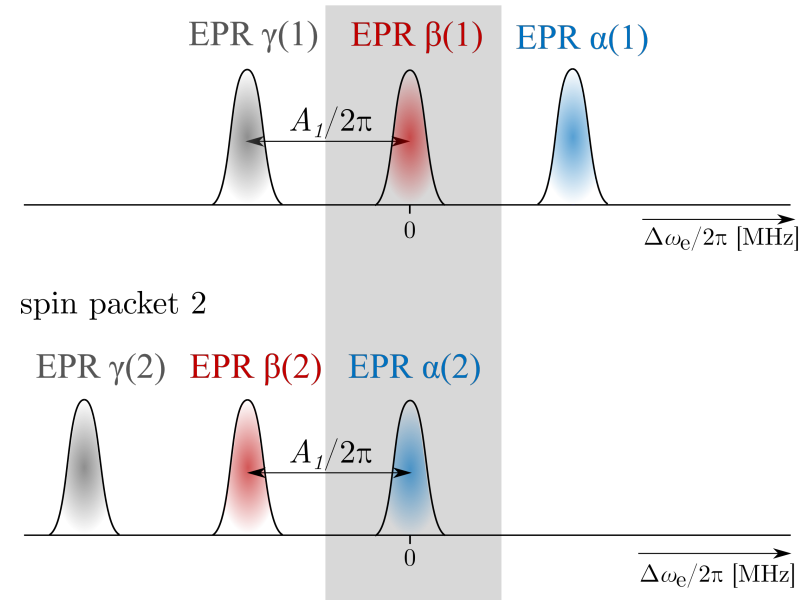

spin packet 3

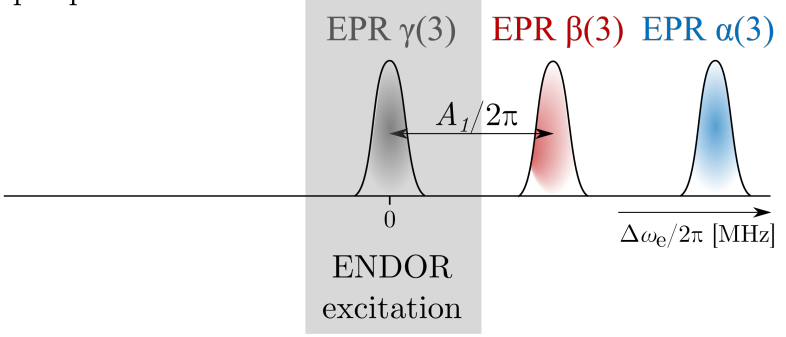

B) CP-ENDOR at ENDOR excitation (match for EPR $\alpha=$ match for $E P R \beta$ )

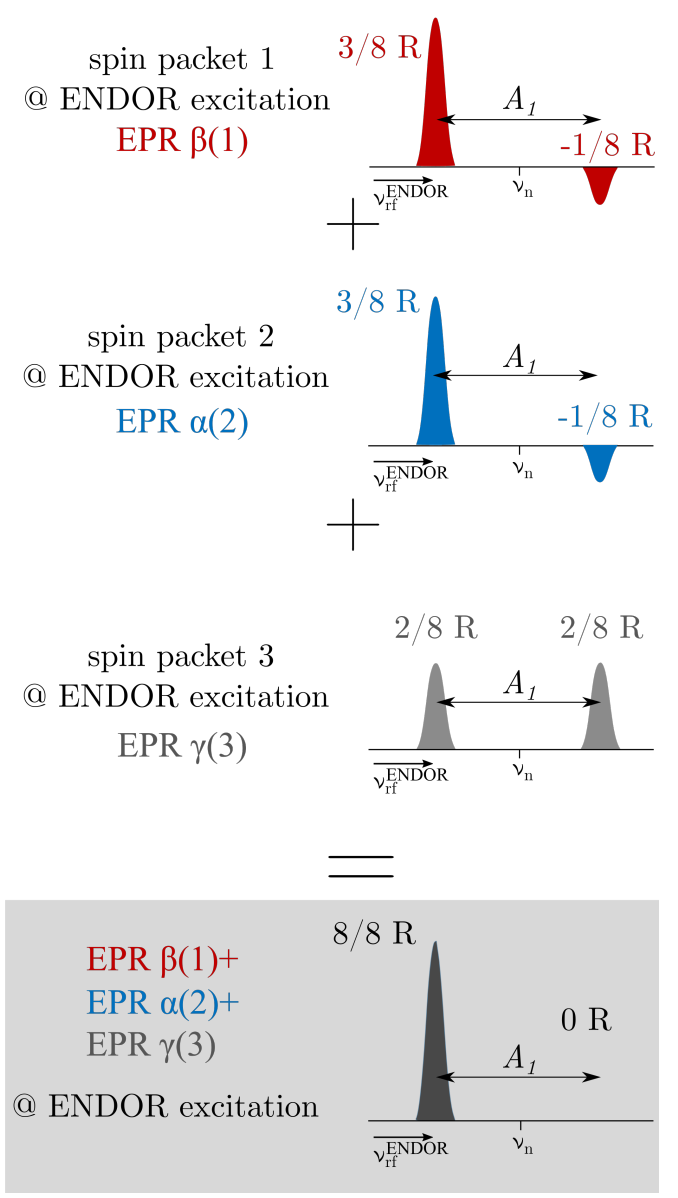

Figure 4.10: Illustration of the calculation strategy for powder pattern CP-ENDOR simulations. A) Example case showing three spin packets with equal hfc $A_{1} / 2 \pi$. In this particular case they are shifted by $A_{1} / 2 \pi$ due to $g$-anisotropy. One EPR transition out of each spin packet is on resonance at the ENDOR excitation position (bottom). B) CP-ENDOR intensity predicted at the ENDOR excitation position. Rf offset during $\mathrm{CP}$ is matched for $\mathrm{EPR} \alpha$, which is in turn also a matching rf offset for EPR $\beta$ (see Table 5.3). The CP-ENDOR intensity at the ENDOR excitation position is obtained from summation of the respective CP-ENDOR at EPR $\alpha, \beta$ and $\gamma$ (bottom). 


\subsubsection{Powder pattern simulations of $\mathrm{ND}_{2} \mathrm{Y}_{731}{ }^{\bullet}$}

The CP-ENDOR simulations of $\mathrm{ND}_{2} \mathrm{Y}_{731}$ required two more extensions of the simulation routine presented in the previous sections. (a) The number of considered nuclear spins for the CP-ENDOR simulation was chosen according to their relevance in the ENDOR spectrum. Since only three deuterium nuclei (D1, D2 and D3) can be identified in the ${ }^{2} \mathrm{H}$-ENDOR spectrum, three ${ }^{2} \mathrm{H}$ nuclear spins are considered by straight-forward matrix expansion (see Appendix B.3). This step increases the density matrix to dimension $54 \times 54(S=1 / 2, I(\mathrm{D} 1)=1, I(\mathrm{D} 2)=1, I(\mathrm{D} 3)=1) . \quad(\mathrm{b})$ The implementation of orientation-selection was done via a pre-routine that considers all coupled nuclei (D1, D2, D3, ${ }^{14} \mathrm{~N}, \mathrm{H}_{\text {Ring }}, \mathrm{H}_{\beta}$ ) following the simulation script developed in-house ${ }^{[13]}$. First, the routine calculates the EPR powder line shape including $g$-anisotropy and all nuclear spins. The script calculates $A_{z z}$ and $Q_{z z}$ according to the selected excitation position in the EPR line (= orientation-selection, see also Subsec. 2.3.4). Then, the obtained hf- and quad-values for D1, D2 and D3 are handed over to the CP-ENDOR simulation which calculates the 2D WOP CP-ENDOR spectrum for the selected $A_{z z}(\mathrm{D} 1, \mathrm{D} 2, \mathrm{D} 3)$ and $Q_{z z}(\mathrm{D} 1, \mathrm{D} 2, \mathrm{D} 3)$ combinations according to the CP simulation script. 



\section{${ }^{2} \mathrm{H}$ eNCP and ${ }^{2} \mathrm{H}$ CP-ENDOR single crystal studies of a deuterated malonic acid radical}

In this chapter, the experimental and theoretical description of eNCP and CP-ENDOR for deuterium nuclei are presented. First, CP matching conditions will be identified from eNCP experiments of a single crystal of deuterated malonic acid radical and theoretical predictions for a $S=1 / 2, I=1$ spin system. Second, CP-ENDOR intensities are going to be discussed. CP-ENDOR intensities will be predicted based on analytical and numerical density matrix calculations and compared with the experimental CP-ENDOR spectra.

\subsection{The $S=1 / 2, I=1$ spin system}

In this description of the eNCP process, the spin system of an electron spin $S=1 / 2$ with $m_{S}= \pm 1 / 2$ coupled to one deuterium $I=1$ with $m_{I}= \pm 1,0$ is investigated. For simplicity of the following treatment HFA was considered, which means that the quantization axis of the electron spin is mainly determined by the electron Zeeman interaction and the influence of the hyperfine interaction on this axis (pseudo-secular hf terms $B_{x}$ and $B_{y}$ ) can be neglected (see also Subsec. 2.3.1) [29]. This is a valid assumption since they are not necessary for driving the CP-mechanism demonstrated in previous published CP-ENDOR treatments 2527 . The quadrupole term is assumed to be axially symmetric $(\eta=0)$ with $\hbar Q=\frac{1}{2} e^{2} q P$ for $I=1$ according to Eq. 2.15 .30]. Following these definitions, the first order Hamiltonian in the laboratory (lab) frame and energy units $\left(H_{0}^{\text {lab }}\right)$ or frequency units $\left(\mathcal{H}_{0}^{\text {lab }}\right)$ is given by

$$
\begin{aligned}
& H_{0}^{\mathrm{lab}}=\mu_{\mathrm{B}} g_{\mathrm{e}} S_{z} B_{0}-\mu_{\mathrm{N}} g_{\mathrm{n}} I_{z} B_{0}+\hbar A S_{z} I_{z}+\hbar Q\left(\frac{3}{2} I_{z}^{2}-\mathbb{1}\right) \\
& \mathcal{H}_{0}^{\mathrm{lab}}=\frac{H_{0}^{\mathrm{lab}}}{\hbar}=\omega_{\mathrm{e}} S_{z}+\omega_{\mathrm{n}} I_{z}+A S_{z} I_{z}+Q\left(\frac{3}{2} I_{z}^{2}-\mathbb{1}\right)
\end{aligned}
$$


where, according to the previous definitions, $\mu_{B}$ and $\mu_{N}$ are the electron and nuclear magnetons respectively, the electron $g$-factor is considered to be isotropic and $\omega_{\mathrm{e}}$ and $\omega_{\mathrm{n}}$ are the Larmor frequencies of electron (e) and nuclear (n) spin, respectively. General spin operators are summarized in Appendix B.1. In Fig. 5.1, A the effect of the hyperfine and quadrupole interaction on the energy level diagram and the EPR spectrum is illustrated for the case of isotropic electron Zeeman interaction, positive hyperfine coupling and quadrupole coupling. In this general case, three EPR transitions denoted as EPR $\alpha, \beta$ and $\gamma$ are observed as shown in Fig. 5.1, B. The case of negative $A / 2 \pi$ and negative $Q / 2 \pi$ is shown in Fig. 5.1, $\mathrm{C}$ and reflects the malonic acid single crystal case. The electronic transitions are denoted as $\operatorname{EPR} \alpha, \operatorname{EPR} \beta$ and $\operatorname{EPR} \gamma$ in blue, red and grey, respectively, as associated to nuclear state $\alpha, \beta$ or $\gamma$. For the following description, an alternative ordering of energy levels in the Hamiltonian when compared to the standard one introduced in Subsec. 2.3.1 of this thesis is introduced. The respective basis transformation is given in Appendix $\mathrm{C}$ and results in

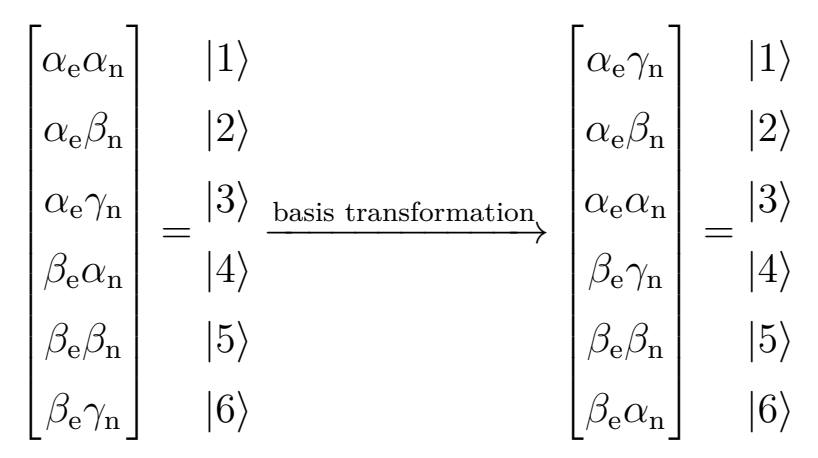

This was done to simplify the assignment of individual energy levels and their corresponding populations in the density matrix in the following treatment, particularly when rewriting the Hamiltonian in the fictitious spin- $1 / 2$ formalism. In the following, the EPR [3-6] ( $\alpha$ ) transition is an electronic spin transition preserving the nuclear spin state $\alpha_{\mathrm{n}}, m_{I}=1\left(\left|\beta_{\mathrm{e}} \alpha_{\mathrm{n}}\right\rangle \rightarrow\left|\alpha_{\mathrm{e}} \alpha_{\mathrm{n}}\right\rangle\right)$, EPR [2-5] $(\beta)$ transition is an electronic spin transition preserving the nuclear spin state $\beta_{\mathrm{n}}, m_{I}=0\left(\left|\beta_{\mathrm{e}} \beta_{\mathrm{n}}\right\rangle \rightarrow\left|\alpha_{\mathrm{e}} \beta_{\mathrm{n}}\right\rangle\right)$ and EPR $[1-4](\gamma)$ is an electronic spin transition preserving the nuclear spin state $\gamma_{\mathrm{n}}, m_{I}=-1$ $\left(\left|\beta_{\mathrm{e}} \gamma_{\mathrm{n}}\right\rangle \rightarrow\left|\alpha_{\mathrm{e}} \gamma_{\mathrm{n}}\right\rangle\right)$. It is recalled from Subsec. 2.3.1, that the number of lines observed in the ENDOR spectrum depends on the probed EPR transition. EPR $\alpha$ is connected with two possible allowed NMR transitions [1-2] and [4-5], resulting in two obtained ENDOR peaks. According to Fig. 5.1 they will be split by the hyperfine and shifted by the quadrupole coupling. Further, for EPR $\gamma$ again two NMR peaks [2-3], [5-6] and for EPR $\beta$ four ENDOR peaks associated with NMR transitions [1-2], [2-3], [4-5] and [5-6] are predicted. 


\section{A) Energy diagram}

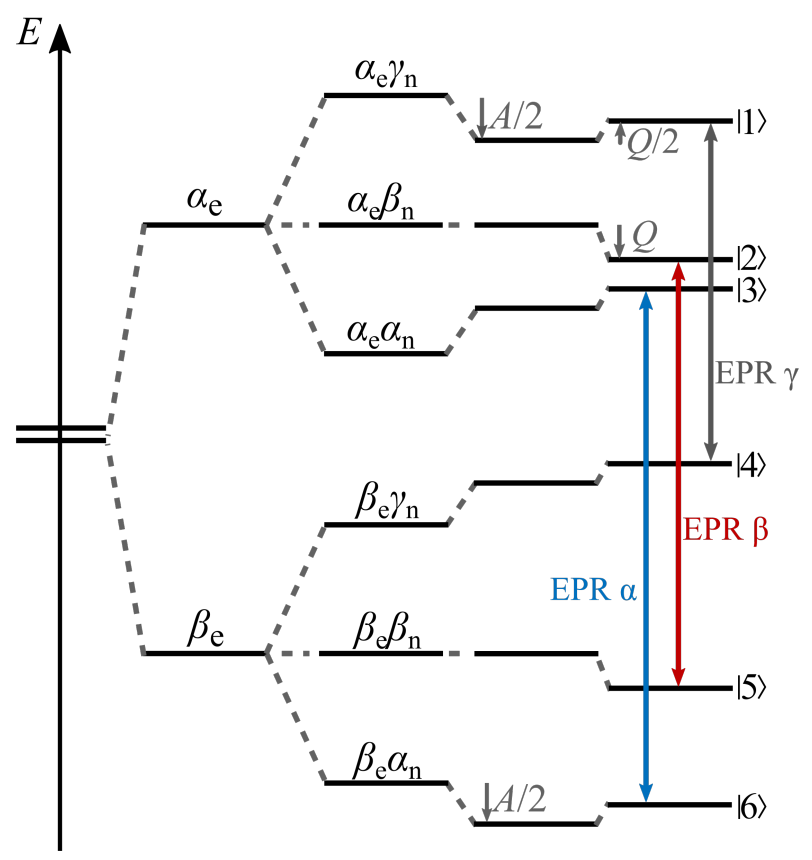

B) EPR resonances

for $A>0$

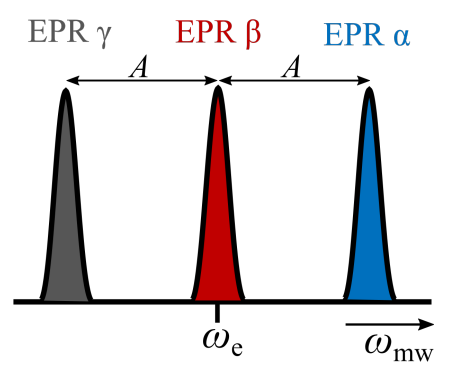

C) EPR resonances for $A<0$

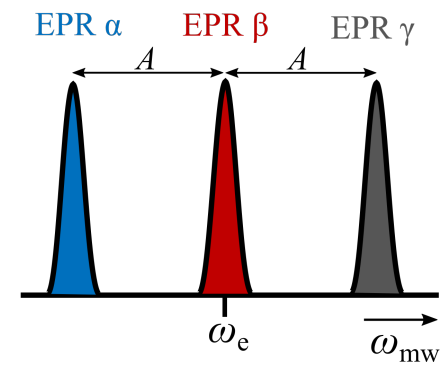

Figure 5.1: First-order energy diagram for an electron spin-1/2 coupled to a nuclear spin-1. A) The energy diagram is shown for the spin system $S=1 / 2, I=1$, $A / 2 \pi>0, A / 2 \pi<\omega_{\mathrm{n}}$ and $Q / 2 \pi>0$. We define $\beta_{\mathrm{e}}=-1 / 2, \alpha_{\mathrm{e}}=+1 / 2, \alpha_{\mathrm{n}}=+1, \beta_{\mathrm{n}}=0$ and $\gamma_{\mathrm{n}}=-1$. B) Line assignment in frequency domain ESE spectrum for $A / 2 \pi>0$, $Q / 2 \pi>0$ (corresponds to the energy diagram shown in A). C) Line assignment in frequency domain EPR spectrum for $A / 2 \pi<0$. Figure adapted from 29 .

One realization of this spin system is a $\gamma$-irradiated single crystal of malonic acid. The stable malonic acid radical is strongly coupled to the central deuterium nucleus (Fig. $5.2 \mathrm{~A}$, green highlight) with well-defined hyperfine $\left(A_{x} / 2 \pi=-4.4 \mathrm{MHz}, A_{y} / 2 \pi=-8.8 \mathrm{MHz}\right.$ and $\left.A_{z} / 2 \pi=-14 \mathrm{MHz}\right)$ and quadrupole tensor values $\left(Q_{x} / 2 \pi=0.07 \mathrm{MHz}\right.$, $Q_{y} / 2 \pi=-0.03 \mathrm{MHz}, Q_{z} / 2 \pi=-0.04 \mathrm{MHz}$. Hyperfine couplings to the carboxyl group deuterons are too small to be resolved. Thus, the echo-detected EPR spectrum at $94 \mathrm{GHz} / 3.4 \mathrm{~T}$ is composed of three lines, separated by a crystal-orientation dependent hyperfine coupling shown in Fig. $5.2 \mathrm{~B}$ ). The crystal was mounted in an orientation with $A / 2 \pi \approx A_{y} / 2 \pi=-8.9 \mathrm{MHz}$ and $Q / 2 \pi \approx Q_{z} / 2 \pi=-0.04 \mathrm{MHz}$. The orientation was chosen for two reasons: 1) $A / 2 \pi=-8.9 \mathrm{MHz}$ is close to the isotropic value $a_{\text {iso }} / 2 \pi=-9.0 \mathrm{MHz}{ }^{88}$, thus, pseudo-secular hyperfine terms can be neglected and the above theoretical assumptions are satisfied. 2) The three EPR lines are well-separated by the sufficiently large $A$, required for selective excitation of each EPR transition $\left(A \gg \omega_{1 \mathrm{e}}\right)$. The sample mounted in this orientation was used for the following eNCP and CP-ENDOR experiments. 


\section{B) ESE-detected \\ field sweep spectrum}

A) Radical structure<smiles>O=C(O)OC(=O)C(=O)O</smiles>

malonic acid radical chemical structure

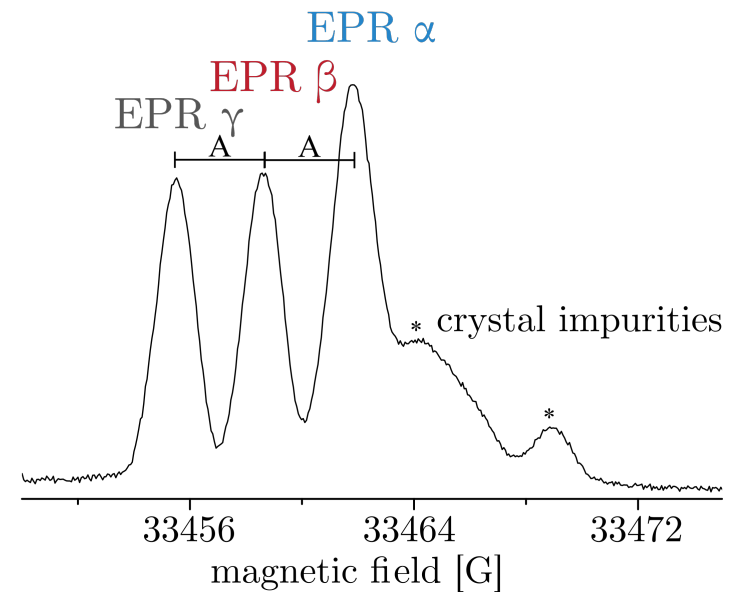

Figure 5.2: Chemical structure of malonic acid and ESE-detected field sweep spectrum. A) Chemical structure of malonic acid. The strongly coupled nucleus is highlighted in green. B) $94 \mathrm{GHz} / 3.4 \mathrm{~T}$ ESE-detected field sweep spectrum of malonic acid. In the chosen orientation is $A \approx A_{y} / 2 \pi=-8.9 \mathrm{MHz}$ and $Q / 2 \pi \approx Q_{z} / 2 \pi=-0.04 \mathrm{MHz}$. Exp. parameters: $T=40 \mathrm{~K}, \nu_{\mathrm{EPR}}=94 \mathrm{GHz}$, $t_{\pi / 2, \mathrm{mw}}=20 \mathrm{~ns}, \tau=500 \mathrm{~ns}, 25 \mathrm{shot} /$ point, $1 \mathrm{scan}$. Note: The EPR spectrum is shown in field domain in contrast to Fig. 5.1, C which interchanges the assignment of the hf-transitions as compared to Fig. 5.1 .

\section{2. eNCP experiments and matching conditions}

$$
\text { for } S=1 / 2, I=1
$$

At first, the matching conditions for the $S=1 / 2, I=1$ spin system were obtained from eNCP experiments on the single crystal, in analogy to previously published procedures for proton spectra (see also Subsec. 2.4.1) 25, 27. The experimental eNCP spectra were recorded by detecting the electron polarization loss for selective excitation of EPR transition as a function of the rf frequency with the sequence shown in Fig. 5.3, A. Assignment of the obtained CP matching conditions resulted from analytical calculations and was supported by numerical simulations. The results are summarized in Fig. 5.3 and will be presented separately in the following subsections. The experimental results are described in Subsection 5.2.1, analytical calculations in Subsection 5.2.2 and numerical simulations in Subsection 5.2.3. 


\section{A) eNCP sequence}

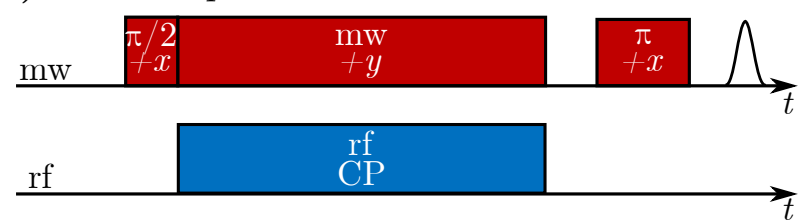

B) eNCP spectra
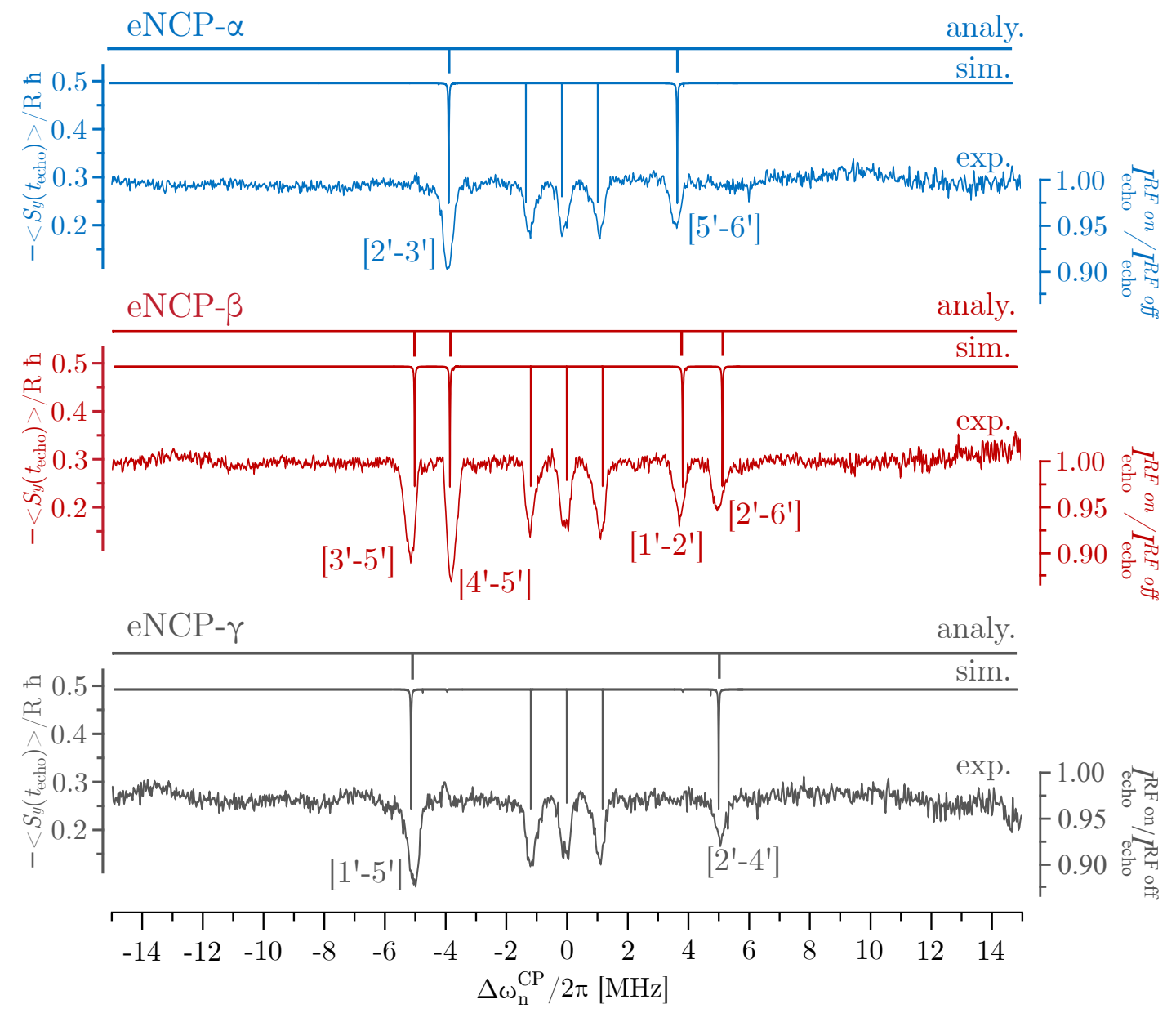

Figure 5.3: eNCP experiments and calculated matching conditions for a deuterated malonic acid single crystal. A) Schematic illustration of the eNCP pulse sequence. B) Experimental eNCP spectra selective for each EPR transition $(\alpha, \beta$ and $\gamma)$ (exp.) and resonances derived from analytical treatment (analy.) and numerical simulations (sim.). In parenthesis are assignments to respective $\mathrm{CP}$ matching conditions. Exp. conditions: $T=40 \mathrm{~K}, \nu_{\mathrm{EPR}}=94 \mathrm{GHz}$, shot repetition time $=100 \mathrm{~ms}, t_{\pi / 2, \mathrm{mw}}=200 \mathrm{~ns}, \omega_{1 \mathrm{e}} / 2 \pi=1.25 \mathrm{MHz}, t_{\mathrm{CP}}=150 \mu \mathrm{s}, \omega_{1 \mathrm{n}} / 2 \pi=16 \mathrm{kHz}$, $\mathrm{rf}_{\text {sweep }}=16 \mathrm{MHz}, 1$ shot $/$ point, random rf acquisition, $100-250$ scans. Sim. parameters: $A(\mathrm{D} 1) / 2 \pi=-0.1 \mathrm{MHz}, A(\mathrm{D} 2) / 2 \pi=-8.9 \mathrm{MHz}, Q(\mathrm{D} 1) / 2 \pi=0.0 \mathrm{MHz}$, $Q(\mathrm{D} 2) / 2 \pi=-0.04 \mathrm{MHz}, \omega_{1 \mathrm{e}} / 2 \pi=1.18 \mathrm{MHz}, \omega_{1 \mathrm{n}} / 2 \pi=10 \mathrm{kHz}, \mathrm{rf}_{\text {sweep }}=30 \mathrm{MHz}$, $\Delta \omega_{\mathrm{e}}= \pm A / 2 \pi, 0$. 


\subsection{1. eNCP experiments}

All experiments were performed with the single crystal mounted in the same orientation $\left(A / 2 \pi \approx A_{y} / 2 \pi=-8.9 \mathrm{MHz}\right.$ and $\left.Q / 2 \pi \approx Q_{z} / 2 \pi=-0.04 \mathrm{MHz}\right)$ and under similar experimental conditions. Experimental parameters are given in the caption of Fig. 5.3. Irradiation on EPR $\alpha$ (Fig. $5.3 \mathrm{~B}$, blue) resulted in depolarization peaks at $\Delta \omega_{\mathrm{n}}^{\mathrm{CP}} / 2 \pi=-3.8,3.9 \mathrm{MHz}$. Irradiation on $\mathrm{EPR} \beta$ (Fig. $5.3 \mathrm{~B}$, red) resulted in peaks at $\Delta \omega_{\mathrm{n}}^{\mathrm{CP}} / 2 \pi=-3.8,3.9 \mathrm{MHz}$ and $-5.1,5.2 \mathrm{MHz}$. Finally, resonances at $\Delta \omega_{\mathrm{n}}^{\mathrm{CP}} / 2 \pi=-5.1,5.2 \mathrm{MHz}$ are obtained for irradiation on EPR $\gamma$ (Fig.5.3B, grey). Additional peaks at $\Delta \omega_{\mathrm{n}}^{\mathrm{CP}} / 2 \pi= \pm 1.18 \mathrm{MHz}$ and $0 \mathrm{MHz}$ were obtained for each of the three experiments and are discussed later.

\subsubsection{Matching conditions from analytical treatment}

The following analytical treatment was elaborated in collaboration with Dr. Roberto Rizzato during his $\mathrm{PhD} / \mathrm{PostDoc}$ at our research group on basis of the procedure published for the proton nuclei 25 .

The matching conditions are obtained from a three step procedure where (a) the CPHamiltonian in doubly rotating frame using single transition operators is defined; (b) the electronic spin part of this Hamiltonian gets diagonalized (transformation into a tilted frame) by considering rf irradiation as a perturbation and; (c) matching conditions are obtained as pairs of degenerate states connected in the tilted frame by a non-diagonal element.

As the following steps directly deal with individual elements and populations of the Hamiltonian in Eq. 5.2, it is convenient and much more suited to convert Eq. 5.2 into a basis $\tilde{\mathcal{H}}$ in which the elements are numbered according to the energy scheme of Fig. 5.2, A. The straight-forward basis transformation and particular transformations of spin operators are given in Appendix C.

In order to derive the $\mathrm{CP}$ matching conditions, an electron spin $S=1 / 2$ coupled to a nucleus with spin $I=1$ with positive, isotropic hfc $A$ and positive gyromagnetic ratio (such as the case for deuterium) is considered. Accordingly, the Hamiltonian in the electron-nuclear doubly rotating frame under mw and rf irradiation is given by

$$
\tilde{\mathcal{H}}=\Delta \omega_{\mathrm{e}} \tilde{S}_{z}+\Delta \omega_{\mathrm{n}} \tilde{I}_{z}+A \tilde{S}_{z} \tilde{I}_{z}+Q\left(\frac{3}{2} \tilde{I}_{z}^{2}-\mathbb{1}\right)+\omega_{1 \mathrm{e}} \tilde{S}_{y}+\omega_{1 \mathrm{n}} \tilde{I}_{x}
$$


with $\omega_{1 \mathrm{e}}=\frac{g_{\mathrm{e}} \mu_{\mathrm{B}} B_{1 \mathrm{e}}}{\hbar}, \omega_{1 \mathrm{n}}=\frac{g_{\mathrm{n}} \mu_{\mathrm{N}} B_{1 \mathrm{n}}}{\hbar}, Q=\frac{1}{2} e^{2} q Q_{z z} / 2 \pi$ and $\Delta \omega_{\mathrm{e}}=\omega_{\mathrm{e}}-\omega_{\mathrm{mw}}, \Delta \omega_{\mathrm{n}}=\omega_{\mathrm{n}}-\omega_{\mathrm{rf}}$ and $\mathbb{1}$ is a $6 \times 6$ identity matrix. It is noted that both, nuclear Larmor $\left(\omega_{\mathrm{n}}\right)$ and $\mathrm{rf}\left(\omega_{\mathrm{rf}}\right)$ are negative, which must be taken into account in the calculation of the eNCP matching conditions and CP-ENDOR intensities. Equation 5.4 can be rewritten in the fictitious spin-1/2 operators for which we use the following definitions 93 94]:

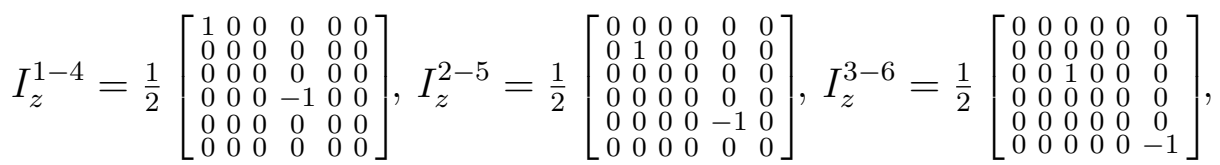

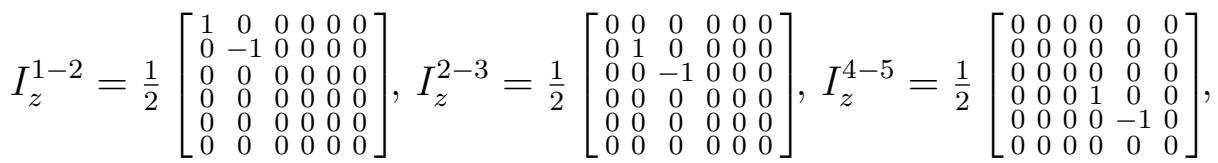

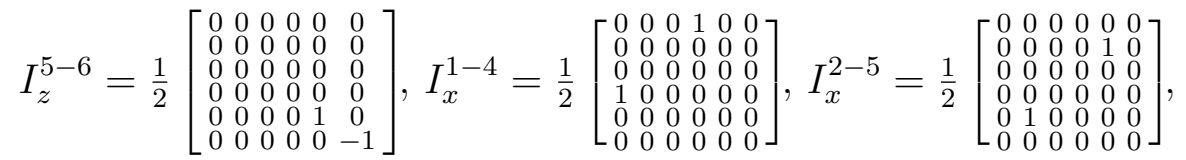

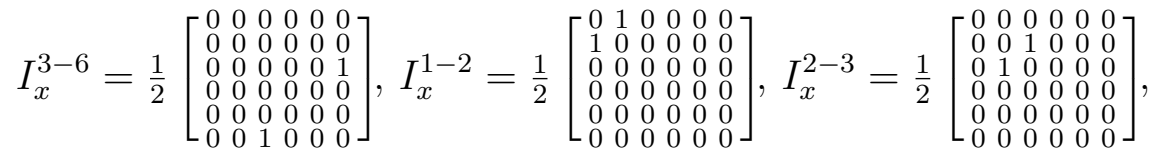

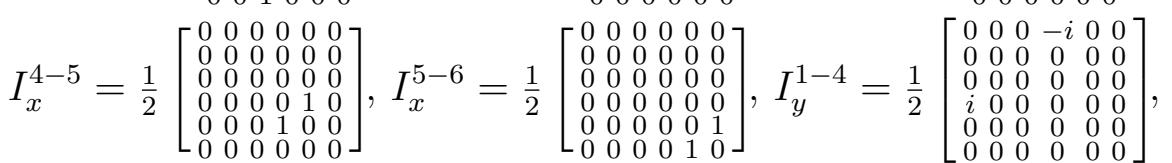

$$
\begin{aligned}
& I_{y}^{2-5}=\frac{1}{2}\left[\begin{array}{cccccc}
0 & 0 & 0 & 0 & 0 & 0 \\
0 & 0 & 0 & 0 & -i & 0 \\
0 & 0 & 0 & 0 & 0 & 0 \\
0 & 0 & 0 & 0 & 0 & 0 \\
0 & i & 0 & 0 & 0 & 0 \\
0 & 0 & 0 & 0 & 0 & 0
\end{array}\right], I_{y}^{3-6}=\frac{1}{2}\left[\begin{array}{cccccc}
0 & 0 & 0 & 0 & 0 & 0 \\
0 & 0 & 0 & 0 & 0 & 0 \\
0 & 0 & 0 & 0 & 0 & - \\
0 & 0 & 0 & 0 & 0 & 0 \\
0 & 0 & 0 & 0 & 0 & 0 \\
0 & 0 & i & 0 & 0 & 0
\end{array}\right]
\end{aligned}
$$

which are used to rewrite the operators

$$
\begin{aligned}
& \tilde{S}_{z}=\frac{1}{2}\left[\begin{array}{cccccc}
1 & 0 & 0 & 0 & 0 & 0 \\
0 & 1 & 0 & 0 & 0 & 0 \\
0 & 0 & 1 & 0 & 0 & 0 \\
0 & 0 & 0 & -1 & 0 & 0 \\
0 & 0 & 0 & 0 & -1 & 0 \\
0 & 0 & 0 & 0 & 0 & -1
\end{array}\right], \tilde{I}_{z}=\left[\begin{array}{cccccc}
-1 & 0 & 0 & 0 & 0 & 0 \\
0 & 0 & 0 & 0 & 0 & 0 \\
0 & 0 & 1 & 0 & 0 & 0 \\
0 & 0 & 0 & -1 & 0 & 0 \\
0 & 0 & 0 & 0 & 0 & 0 \\
0 & 0 & 0 & 0 & 0 & 1
\end{array}\right], \\
& \tilde{S}_{x}=\frac{1}{2}\left[\begin{array}{llllll}
0 & 0 & 0 & 1 & 0 & 0 \\
0 & 0 & 0 & 0 & 1 & 0 \\
0 & 0 & 0 & 0 & 0 & 1 \\
1 & 0 & 0 & 0 & 0 & 0 \\
0 & 1 & 0 & 0 & 0 & 0 \\
0 & 0 & 1 & 0 & 0 & 0
\end{array}\right], \tilde{I}_{x}=\frac{1}{\sqrt{2}}\left[\begin{array}{llllll}
0 & 1 & 0 & 0 & 0 & 0 \\
1 & 0 & 1 & 0 & 0 & 0 \\
0 & 1 & 0 & 0 & 0 & 0 \\
0 & 0 & 0 & 0 & 1 & 0 \\
0 & 0 & 0 & 1 & 0 & 1 \\
0 & 0 & 0 & 0 & 1 & 0
\end{array}\right], \tilde{S}_{y}=\frac{1}{2}\left[\begin{array}{cccccc}
0 & 0 & 0 & -i & 0 & 0 \\
0 & 0 & 0 & 0 & -i & 0 \\
0 & 0 & 0 & 0 & 0 & -i \\
i & 0 & 0 & 0 & 0 & 0 \\
0 & i & 0 & 0 & 0 & 0 \\
0 & 0 & i & 0 & 0 & 0
\end{array}\right],
\end{aligned}
$$


into

$$
\begin{aligned}
& \tilde{S}_{z}=I_{z}^{1-4}+I_{z}^{2-5}+I_{z}^{3-6} \\
& \tilde{I}_{z}=2\left(-I_{z}^{1-2}-I_{z}^{2-3}-I_{z}^{4-5}-I_{z}^{5-6}\right) \\
& \tilde{S}_{x}=I_{x}^{1-4}+I_{x}^{2-5}+I_{x}^{3-6} \\
& \tilde{S}_{y}=I_{y}^{1-4}+I_{y}^{2-5}+I_{y}^{3-6} \\
& \tilde{I}_{x}=\sqrt{2}\left(I_{x}^{1-2}+I_{x}^{2-3}+I_{x}^{4-5}+I_{x}^{5-6}\right) \\
& A \tilde{S}_{z} \tilde{I}_{z}=A\left(I_{z}^{1-4}+I_{z}^{2-5}+I_{z}^{3-6}\right) 2\left(-I_{z}^{1-2}-I_{z}^{2-3}-I_{z}^{4-5}-I_{z}^{5-6}\right)
\end{aligned}
$$

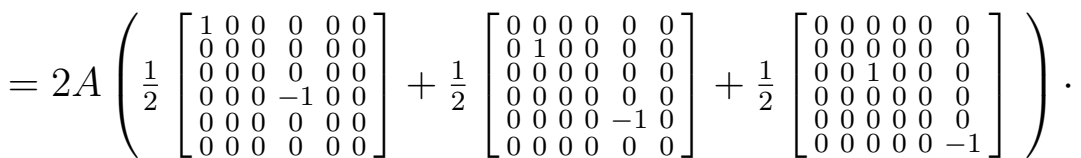

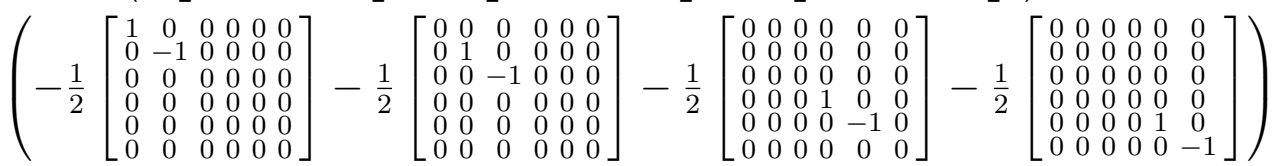

$$
\begin{aligned}
& =2 A\left[\begin{array}{cccccc}
1 / 2 & 0 & 0 & 0 & 0 & 0 \\
0 & 1 / 2 & 0 & 0 & 0 & 0 \\
0 & 0 & 1 / 2 & 0 & 0 & 0 \\
0 & 0 & 0 & -1 / 2 & 0 & 0 \\
0 & 0 & 0 & 0 & -1 / 2 & 0 \\
0 & 0 & 0 & 0 & 0 & -1 / 2
\end{array}\right] \cdot\left[\begin{array}{cccccc}
-1 / 2 & 0 & 0 & 0 & 0 & 0 \\
0 & 0 & 0 & 0 & 0 & 0 \\
0 & 0 & 1 / 2 & 0 & 0 & 0 \\
0 & 0 & 0 & -1 / 2 & 0 & 0 \\
0 & 0 & 0 & 0 & 0 & 0 \\
0 & 0 & 0 & 0 & 0 & 1 / 2
\end{array}\right]=A\left[\begin{array}{cccccc}
-1 / 2 & 0 & 0 & 0 & 0 & 0 \\
0 & 0 & 0 & 0 & 0 & 0 \\
0 & 0 & 1 / 2 & 0 & 0 & 0 \\
0 & 0 & 0 & 1 / 2 & 0 & 0 \\
0 & 0 & 0 & 0 & 0 & 0 \\
0 & 0 & 0 & 0 & 0 & -1 / 2
\end{array}\right] \\
& =A\left(-I_{z}^{1-4}+I_{z}^{3-6}\right)
\end{aligned}
$$

and

$$
\begin{aligned}
& Q\left(\frac{3}{2} \tilde{I}_{z}^{2}-\mathbb{1}\right)=Q\left(\frac{3}{2}\left(-2 I_{z}^{1-2}-2 I_{z}^{2-3}-2 I_{z}^{4-5}-2 I_{z}^{5-6}\right)^{2}-\mathbb{1}\right)
\end{aligned}
$$

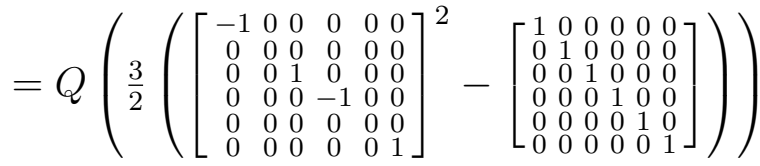

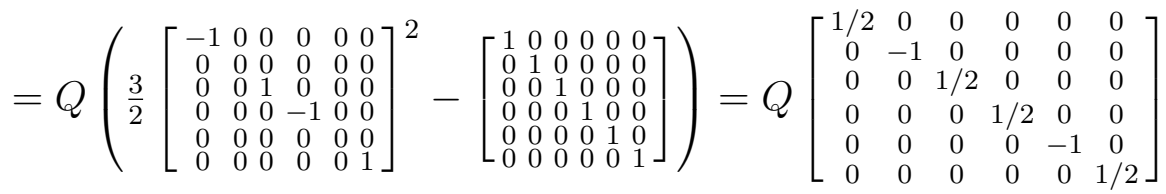

$$
\begin{aligned}
& =Q\left(I_{z}^{1-2}-I_{z}^{2-3}+I_{z}^{4-5}-I_{z}^{5-6}\right) .
\end{aligned}
$$

Thus, the Hamiltonian in Eq. 5.4 can be rewritten as

$$
\begin{aligned}
\tilde{\mathcal{H}} & =\underbrace{\left(\Delta \omega_{\mathrm{e}}-A\right) I_{z}^{1-4}}_{\mathrm{EPR} \gamma}+\underbrace{\Delta \omega_{\mathrm{e}} I_{z}^{2-5}}_{\mathrm{EPR} \beta}+\underbrace{\left(\Delta \omega_{\mathrm{e}}+A\right) I_{z}^{3-6}}_{\mathrm{EPR} \alpha} \\
& -2 \Delta \omega_{\mathrm{n}}\left(I_{z}^{1-2}+I_{z}^{2-3}+I_{z}^{4-5}+I_{z}^{5-6}\right)+Q\left(I_{z}^{1-2}-I_{z}^{2-3}+I_{z}^{4-5}-I_{z}^{5-6}\right) \\
& +\omega_{1 \mathrm{e}}\left(I_{y}^{1-4}+I_{y}^{2-5}+I_{y}^{3-6}\right)+\omega_{1 \mathrm{n}} \sqrt{2}\left(I_{x}^{1-2}+I_{x}^{2-3}+I_{x}^{4-5}+I_{x}^{5-6}\right) .
\end{aligned}
$$


This rewriting allows for separate treatment of the electron and nuclear contributions of the Hamiltonian.

$$
\begin{aligned}
\tilde{\mathcal{H}} & =\tilde{\mathcal{H}}_{\mathrm{e}}+\tilde{\mathcal{H}}_{\mathrm{n}} \\
\tilde{\mathcal{H}}_{e} & =\left(\Delta \omega_{\mathrm{e}}-A\right) I_{z}^{1-4}+\Delta \omega_{\mathrm{e}} I_{z}^{2-5}+\left(\Delta \omega_{\mathrm{e}}+A\right) I_{z}^{3-6} \\
& +\omega_{1 \mathrm{e}}\left(I_{y}^{1-4}+I_{y}^{2-5}+I_{y}^{3-6}\right) \\
\tilde{\mathcal{H}}_{\mathrm{n}} & =-2 \Delta \omega_{\mathrm{n}}\left(I_{z}^{1-2}+I_{z}^{2-3}+I_{z}^{4-5}+I_{z}^{5-6}\right)+Q\left(I_{z}^{1-2}-I_{z}^{2-3}+I_{z}^{4-5}-I_{z}^{5-6}\right) \\
& +\omega_{1 \mathrm{n}} \sqrt{2}\left(I_{x}^{1-2}+I_{x}^{2-3}+I_{x}^{4-5}+I_{x}^{5-6}\right) .
\end{aligned}
$$

In this analytical treatment only the electron part of the Hamiltonian is diagonalized as the nuclear part is considered as a perturbation. The frame where $\tilde{\mathcal{H}}_{\mathrm{e}}$ is diagonal is further called tilted frame. In this frame, the electron spin is quantized along the effective field $\omega$ defined by the electron offset $\Delta \omega_{\mathrm{e}}$, the hyperfine coupling $A$ and mw field strength $\omega_{1 \mathrm{e}}$, as illustrated in Figure 5.4 .

The transformation into the tilted frame is performed via

$$
U_{x} \tilde{\mathcal{H}}_{e} U_{x}^{-1}
$$

with $U_{x}$ defined as

$$
\begin{aligned}
& U_{x}\left(\theta_{\gamma}, \theta_{\beta}, \theta_{\alpha}\right)=\exp \left(-i \theta_{\gamma} I_{x}^{1-4}\right) \exp \left(-i \theta_{\beta} I_{x}^{2-5}\right) \exp \left(-i \theta_{\alpha} I_{x}^{3-6}\right) \\
& =\left[\begin{array}{cccccc}
\cos \frac{\theta_{\gamma}}{2} & 0 & 0 & -i \sin \frac{\theta_{\gamma}}{2} & 0 & 0 \\
0 & \cos \frac{\theta_{\beta}}{2} & 0 & 0 & -i \sin \frac{\theta_{\beta}}{2} & 0 \\
0 & 0 & \cos \frac{\theta_{\alpha}}{2} & 0 & 0 & -i \sin \frac{\theta_{\alpha}}{2} \\
-i \sin \frac{\theta_{\gamma}}{2} & 0 & 0 & \cos \frac{\theta_{\gamma}}{2} & 0 & 0 \\
0 & -i \sin \frac{\theta_{\beta}}{2} & 0 & 0 & \cos \frac{\theta_{\beta}}{2} & 0 \\
0 & 0 & -i \sin \frac{\theta_{\alpha}}{2} & 0 & 0 & \cos \frac{\theta_{\alpha}}{2}
\end{array}\right] .
\end{aligned}
$$

The three angles $\theta_{\alpha, \beta, \gamma}$ individually act on one of the electron manifolds based on the assumption of selective EPR excitation. The angles are defined according to the orientation of the $z$-axis laboratory frame of each manifold relative to the orientation of the tilted magnetization during selective irradiation (= effective field orientations). In order to visualize this transformation, it is useful to consider the direction of the effective magnetic field in each of the electronic manifolds separately. Thus, $U_{x} \tilde{\mathcal{H}} U_{x}^{-1}$ transforms the direction of the z-laboratory frame axis of each electron manifold independently to coincide with the effective field direction $\theta_{\alpha, \beta, \gamma}$ (see Fig. 5.4). The transformation does not influence the diagonal part of the nuclear Hamiltonian, but it 
contributes to split the eigenstates in the electronic manifolds by some constant nuclear offset $-2 \Delta \omega_{\mathrm{n}}\left(I_{z}^{1-2}+I_{z}^{2-3}+I_{z}^{4-5}+I_{z}^{5-6}\right)+Q\left(I_{z}^{1-2}-I_{z}^{2-3}+I_{z}^{4-5}-I_{z}^{5-6}\right)$ and rotates the nuclear non-diagonal part.

A) General case

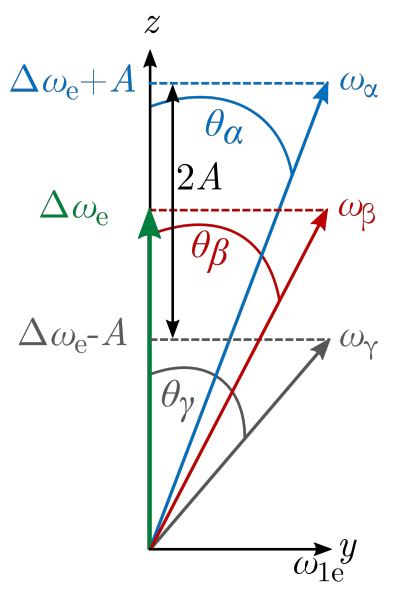

C) On resonance with EPR $\beta$

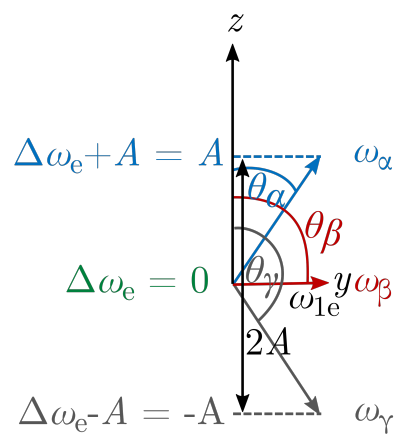

B) On resonance with EPR $\alpha$

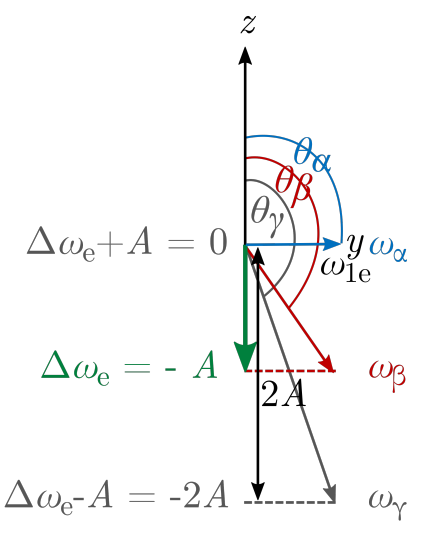

D) On resonance with EPR $\gamma$

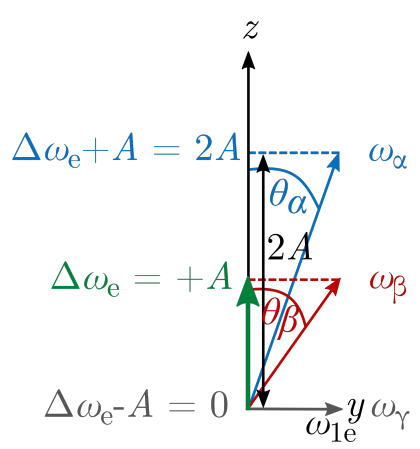

Figure 5.4: Illustration of the rotation angles used to transfer the Hamiltonian into the tilted frame. The rotation angles $\theta_{\alpha}, \theta_{\beta}$ and $\theta_{\gamma}$ are used in the unitary transformation $U_{x}\left(\theta_{\gamma}, \theta_{\beta}, \theta_{\alpha}\right)$ to transfer the Hamiltonian into a frame in which the $z$ direction is defined by the effective fields. A) General case. B) On resonance irradiation with EPR $\alpha$ manifold, $C$ ) EPR $\beta$ manifold and $D$ ) EPR $\gamma$ manifold. The rotation angles are defined according to Table 5.1. (Note: Shown for the case of $A / 2 \pi>0$.)

The rotation angles $\theta_{\alpha}, \theta_{\beta}, \theta_{\gamma}$ for each of the manifolds are obtained according to trigonometric tangent functions in Fig. 5.4 are given in Table 5.1. 
Table 5.1: Rotation angles for each EPR manifold. The rotation angles $\theta_{\alpha}, \theta_{\beta}, \theta_{\gamma}$ are listed for the case of $A / 2 \pi>0$.

$$
\begin{aligned}
& \text { for } \Delta \omega_{\mathrm{e}}-A \geq 0 \\
& \theta_{\gamma}=\tan ^{-1}\left(\frac{\omega_{1 \mathrm{e}}}{\Delta \omega_{\mathrm{e}}-A}\right) \\
& \text { else } \\
& \theta_{\gamma}=\pi+\tan ^{-1}\left(\frac{\omega_{1 \mathrm{e}}}{\Delta \omega_{\mathrm{e}}-A}\right) \\
& \text { for } \Delta \omega_{\mathrm{e}} \geq 0 \\
& \theta_{\beta}=\tan ^{-1}\left(\frac{\omega_{1 \mathrm{e}}}{\Delta \omega_{\mathrm{e}}}\right) \\
& \theta_{\beta}=\pi+\tan ^{-1}\left(\frac{\omega_{1 \mathrm{e}}}{\Delta \omega_{\mathrm{e}}}\right) \\
& \theta_{\alpha}=\tan ^{-1}\left(\frac{\omega_{1 \mathrm{e}}}{\Delta \omega_{\mathrm{e}}+A}\right)
\end{aligned}
$$

The resulting electronic part of the Hamiltonian in the tilted frame is

$$
\begin{aligned}
\tilde{\mathcal{H}}_{\mathrm{e}}^{\prime} & =\sqrt{\left(\Delta \omega_{\mathrm{e}}-A\right)^{2}+\omega_{1 \mathrm{e}}^{2}} I_{z}^{1-4} \\
& +\sqrt{\Delta \omega_{\mathrm{e}}^{2}+\omega_{1 \mathrm{e}}^{2}} I_{z}^{2-5} \\
& +\sqrt{\left(\Delta \omega_{\mathrm{e}}+A\right)^{2}+\omega_{1 \mathrm{e}}^{2}} I_{z}^{3-6} \\
& =\omega_{\gamma} I_{z}^{1-4}+\omega_{\beta} I_{z}^{2-5}+\omega_{\alpha} I_{z}^{3-6}
\end{aligned}
$$

with $\omega_{\gamma}=\sqrt{\left(\Delta \omega_{\mathrm{e}}-A\right)^{2}+\omega_{1 \mathrm{e}}^{2}}, \omega_{\beta}=\sqrt{\Delta \omega_{\mathrm{e}}^{2}+\omega_{1 \mathrm{e}}^{2}}$ and $\omega_{\alpha}=\sqrt{\left(\Delta \omega_{\mathrm{e}}+A\right)^{2}+\omega_{1 \mathrm{e}}^{2}}$.

And the diagonal elements are:

$$
\begin{aligned}
& E_{\left|1^{\prime}\right\rangle}=+\frac{1}{2} \omega_{\gamma}-\Delta \omega_{\mathrm{n}}+\frac{1}{2} Q \\
& E_{\left|2^{\prime}\right\rangle}=+\frac{1}{2} \omega_{\beta}-Q \\
& E_{\left|3^{\prime}\right\rangle}=+\frac{1}{2} \omega_{\alpha}+\Delta \omega_{\mathrm{n}}+\frac{1}{2} Q \\
& E_{\left|4^{\prime}\right\rangle}=-\frac{1}{2} \omega_{\gamma}-\Delta \omega_{\mathrm{n}}+\frac{1}{2} Q \\
& E_{\left|5^{\prime}\right\rangle}=-\frac{1}{2} \omega_{\beta}-Q \\
& E_{\left|6^{\prime}\right\rangle}=-\frac{1}{2} \omega_{\alpha}+\Delta \omega_{\mathrm{n}}+\frac{1}{2} Q
\end{aligned}
$$

with $\omega_{\alpha}, \omega_{\beta}$ and $\omega_{\gamma}$ as defined before.

The off-diagonal elements of the tilted frame Hamiltonian are generally small with respect to their connecting diagonal element and proportional to $\omega_{1 \mathrm{n}}$ as derived from their analytically computation of $\omega_{1 \mathrm{n}} \sqrt{2} U\left[I_{x}^{1-2}+I_{x}^{2-3}+I_{x}^{4-5}+I_{x}^{5-6}\right] U^{-1}$ (not shown). Because the efficiency during the CP step depends on the magnitude of these off-diagonal elements there is no $\mathrm{CP}$ effect as long as they are small as compare to the diagonal elements. The CP condition is described as when pairs of levels $\left[i^{\prime}-j^{\prime}\right]$ in the tilted frame become degenerate and there is an off-diagonal element that no longer can be 
ignored. Thus, it becomes source of a CP process that transfers population until they are equalized. The matching conditions are obtained from setting always two of the diagonal elements (Eq. 5.26-5.31) equal, which are connected by an always non-zero off-diagonal element (numerically calculated, not shown), and reformulate as expressing the equality as a function of $\Delta \omega_{\mathrm{n}}^{\mathrm{CP}}$. The possible matching conditions are:

$$
\begin{aligned}
& \Delta \omega_{\mathrm{n}}^{\mathrm{CP}\left[1^{\prime}-5^{\prime}\right]}=\frac{+\omega_{\gamma}+\omega_{\beta}+3 Q}{2} \quad \Delta \omega_{\mathrm{n}}^{\mathrm{CP}\left[2^{\prime}-6^{\prime}\right]}=\frac{+\omega_{\beta}+\omega_{\alpha}+3 Q}{2} \\
& \Delta \omega_{\mathrm{n}}^{\mathrm{CP}}\left[1^{\prime}-2^{\prime}\right]=\frac{+\omega_{\gamma}-\omega_{\beta}+3 Q}{2} \quad \Delta \omega_{\mathrm{n}}^{\mathrm{CP}\left[5^{\prime}-6^{\prime}\right]}=\frac{-\omega_{\beta}+\omega_{\alpha}+3 Q}{2} \\
& \Delta \omega_{\mathrm{n}}^{\mathrm{CP}\left[4^{\prime}-5^{\prime}\right]}=\frac{-\omega_{\gamma}+\omega_{\beta}+3 Q}{2} \quad \Delta \omega_{\mathrm{n}}^{\mathrm{CP}\left[2^{\prime}-3^{\prime}\right]}=\frac{+\omega_{\beta}-\omega_{\alpha}+3 Q}{2} \\
& \Delta \omega_{\mathrm{n}}^{\mathrm{CP}\left[2^{\prime}-4^{\prime}\right]}=\frac{-\omega_{\gamma}-\omega_{\beta}+3 Q}{2} \quad \Delta \omega_{\mathrm{n}}^{\mathrm{CP}}\left[3^{\prime}-5^{\prime}\right]=\frac{-\omega_{\beta}-\omega_{\alpha}+3 Q}{2}
\end{aligned}
$$

The matching conditions are further calculated for the malonic acid single crystal case. We note again that both the hfc and quadrupole coupling have negative signs which changes the resonance conditions of the EPR lines as illustrated in Fig. 5.1, $B \rightarrow C$. Hfc $(A / 2 \pi=-8.9 \mathrm{MHz})$ and quadrupole coupling $(Q / 2 \pi=-0.04 \mathrm{MHz})$ were defined in the first section and the microwave field strength $\omega_{1 \mathrm{e}} / 2 \pi=1.18 \mathrm{MHz}$ was set according to the experimental value. For the malonic acid case of $|A / 2 \pi| \gg \omega_{1 \mathrm{e}} / 2 \pi, A / 2 \pi<0$ we obtain a set of rotation angles $\theta_{\alpha}, \theta_{\beta}, \theta_{\gamma}$ summarized in Table 5.2 .

Table 5.2: Turning angles for the case of deuterated malonic acid. Hyperfine and quadrupole coupling are $A / 2 \pi=-8.9 \mathrm{MHz}, Q / 2 \pi=-0.04 \mathrm{MHz}$. Note: In this case $|A / 2 \pi| \gg \omega_{1 \mathrm{e}} / 2 \pi, A / 2 \pi<0$ which refers to the case shown in Fig. 5.4. C).

\begin{tabular}{c|c|c|c} 
& $\operatorname{EPR} \alpha[3-6]$ & $\operatorname{EPR} \beta[2-5]$ & $\operatorname{EPR} \gamma[1-4]$ \\
\hline$\theta_{\alpha}$ & $\pi / 2$ & $\pi$ & $\pi$ \\
$\theta_{\beta}$ & 0 & $\pi / 2$ & $\pi$ \\
$\theta_{\gamma}$ & 0 & 0 & $\pi / 2$
\end{tabular}

The resulting so-called CP-effective matching conditions for selective EPR excitation and detection are obtained from the following steps: 1) All matching conditions according to Eq. 5.32 for the given $A / 2 \pi, Q / 2 \pi$ and $\omega_{1 \mathrm{e}} / 2 \pi$ are calculated as summarized in Table 5.3. The calculation considers that the nuclear Larmor frequency $\omega_{\mathrm{n}}$ and $\omega_{\mathrm{rf}}^{\mathrm{CP}}$ in $\Delta \omega_{\mathrm{n}}^{\mathrm{CP}}$ (Eq. 5.32) are negative. 2) The magnitude of the off-diagonal elements in the tilted frame Hamiltonian are numerically inspected (not shown) for all three sets of euler angles given in Table. 5.2. 
Table 5.3: Analytically calculated matching conditions for the malonic acid case. $A / 2 \pi=-8.9 \mathrm{MHz}, Q / 2 \pi=-0.04 \mathrm{MHz}$ and $\omega_{1 \mathrm{e}} / 2 \pi=1.25 \mathrm{MHz}$. Note that the nuclear Larmor frequency $\omega_{\mathrm{n}}$ and $\omega_{\mathrm{rf}}^{\mathrm{CP}}$ in $\Delta \omega_{\mathrm{n}}^{\mathrm{CP}}$ (Eq. 5.32) are considered negative.

\begin{tabular}{c||c|c|c} 
degenerated & $\operatorname{EPR} \alpha[3-6]$ & $\operatorname{EPR} \beta[2-5]$ & $\operatorname{EPR} \gamma[1-4]$ \\
states & $\Delta \omega_{\mathrm{e}}=-A / 2 \pi$ & $\Delta \omega_{\mathrm{e}}=0$ & $\Delta \omega_{\mathrm{e}}=A / 2 \pi$ \\
\cline { 2 - 4 } & $\Delta \omega_{\mathrm{n}}^{\mathrm{CP}} / 2 \pi[\mathrm{MHz}]$ & $\Delta \omega_{\mathrm{n}}^{\mathrm{CP}} / 2 \pi[\mathrm{MHz}]$ & $\Delta \omega_{\mathrm{n}}^{\mathrm{CP}} / 2 \pi[\mathrm{MHz}]$ \\
\hline \hline$\left[1^{\prime}-2^{\prime}\right]$ & -4.4 & 3.9 & 3.9 \\
{$\left[1^{\prime}-5^{\prime}\right]$} & -13.4 & -5.1 & -5.1 \\
{$\left[2^{\prime}-3^{\prime}\right]$} & -3.8 & 3.9 & 4.5 \\
{$\left[2^{\prime}-4^{\prime}\right]$} & 13.5 & 5.2 & 5.2 \\
{$\left[2^{\prime}-6^{\prime}\right]$} & -5.1 & 5.2 & -13.4 \\
{$\left[3^{\prime}-5^{\prime}\right]$} & 5.2 & -5.1 & 13.5 \\
{$\left[4^{\prime}-5^{\prime}\right]$} & 4.5 & -3.8 & -3.8 \\
{$\left[5^{\prime}-6^{\prime}\right]$} & 3.9 & -3.8 & -4.4
\end{tabular}

Some of the listed matching conditions are connected by only very small off-diagonal elements. For example we find on-resonant with $\operatorname{EPR} \alpha\left(\theta_{\alpha}=\pi / 2, \theta_{\beta}=0, \theta_{\gamma}=0\right)$ that $\mathrm{CP}\left[1^{\prime}-5^{\prime}\right]$ and $\mathrm{CP}\left[2^{\prime}-4^{\prime}\right]$ are only weakly allowed transitions as compared to $\mathrm{CP}\left[2^{\prime}-3^{\prime}\right]$ and $\mathrm{CP}\left[5^{\prime}-6^{\prime}\right]$ (one order of magnitude difference in transition probability). Accordingly, one requirement for a so-called efficient matching conditions is a non-negligible transition probabilities. 3) Some of the matching conditions degenerate energy levels which are already equally populated. Here, CP is non-efficient because it has no net effect on the population. Thus, a second criteria for efficient CP is that the populations of the matched states before CP must be different. This will become more evident from the density-operator formalism presented later in Sec. 5.3.2, e.g. Eq. 5.46. Finally, only the highlighted matching conditions satisfy these conditions and are expected to efficiently drive electron-nuclear cross polarization.

According to this analytical treatment, in total eight effective matching conditions are predicted. For the specific malonic acid parameters, two matching conditions at EPR $\alpha$ $\left(\Delta \omega_{\mathrm{n}}^{\mathrm{CP}} / 2 \pi=-3.8,+3.9 \mathrm{MHz}\right)$, four at $\operatorname{EPR} \beta\left(\Delta \omega_{\mathrm{n}}^{\mathrm{CP}} / 2 \pi=-5.1,-3.8,+3.9,+5.2 \mathrm{MHz}\right)$ and another two at $\operatorname{EPR} \gamma\left(\Delta \omega_{\mathrm{n}}^{\mathrm{CP}} / 2 \pi=-5.1,+5.2 \mathrm{MHz}\right)$ are predicted. The resonance positions are sketched as bars and corresponding energy levels are marked as $\left[i^{\prime}-j^{\prime}\right]$ in the plots of Fig. 5.3 , B. 


\subsubsection{Matching conditions from numerical simulations}

In this thesis, a simulation routine was developed based on density operator formalism in order to simulate eNCP and CP-ENDOR spectra. The build-up was supported by a collaboration with Dr. Robert Zeier from Prof. Dr. Steffen Glaser's research group at TU Munich and is part of the achievements of the this PhD project. The general formalism and simulation strategy are summarized in Sections 4.3.1 of materials and methods. This structure is chosen in order to provide the reader a collective overview not only on the simulation relevant for this chapter, but also for the later extensions to the simulation script.

Consequently in the following section the eNCP simulation results are summarized. For the deuterated malonic acid parameters of $A / 2 \pi=-8.9 \mathrm{MHz}, Q / 2 \pi=-0.04 \mathrm{MHz}$ and $\omega_{1 \mathrm{e}} / 2 \pi=1.18 \mathrm{MHz}$ two lines on the EPR $\alpha$ transition are simulated at $\Delta \omega_{\mathrm{n}}^{\mathrm{CP}} / 2 \pi$ $=-3.8,+3.9 \mathrm{MHz}$. Four lines are obtained at $\operatorname{EPR} \beta\left(\Delta \omega_{\mathrm{n}}^{\mathrm{CP}} / 2 \pi=-5.1,-3.8,+3.9\right.$, $+5.2 \mathrm{MHz})$ and further two at $\operatorname{EPR} \gamma\left(\Delta \omega_{\mathrm{n}}^{\mathrm{CP}} / 2 \pi=-5.2,+5.2 \mathrm{MHz}\right)$, see Fig. $5.3(\operatorname{sim})$. The values are in agreement with the experimental and analytical results.

The peaks observed at the center of each eNCP spectrum (at $\Delta \omega_{\mathrm{n}}^{\mathrm{CP}} / 2 \pi= \pm 1.18 \mathrm{MHz}$ and $0 \mathrm{MHz}$ ) were simulated with parameters of $A / 2 \pi=-0.1 \mathrm{MHz}, Q / 2 \pi=-0.0 \mathrm{MHz}$ and $\omega_{1 \mathrm{e}} / 2 \pi=1.18 \mathrm{MHz}$. These peaks at $\Delta \omega_{\mathrm{n}}^{\mathrm{CP}} / 2 \pi= \pm 1.18 \mathrm{MHz}$ were obtained consistently on $\operatorname{EPR} \alpha, \beta$ and $\gamma$. The results for $A / 2 \pi=0.1 \mathrm{MHz}$ and $A / 2 \pi=-8.9 \mathrm{MHz}$ of each EPR transition were summed up in order to obtain the spectra shown in Fig. 5.3, B.

\subsection{CP-ENDOR experiments and calculations of CP-ENDOR intensities}

The determined matching conditions are now used to set a fixed rf in order to drive electron-nuclear cross polarization. Afterwards, a second RF read-out pulse which transfers nuclear polarization back into observable electron polarization is applied. Finally, the CP-ENDOR spectrum is observed from EPR echo detection as a function of the second RF. Fig. 5.5 summarizes the experimental as well as theoretical results. CP-ENDOR experiments were performed for selective excitation of EPR $\alpha(\mathrm{B}), \mathrm{EPR} \beta$ (C) and EPR $\gamma(\mathrm{D})$. The plots show results for two or four rf offset values with effective matching and one non-matched rf offset for each of the manifolds. The experiments are 
further discussed in Section 5.3 and theoretical results on the basis of analytical and numerical calculations as described in Subsections 5.3 .2 and 5.3.3, respectively.

\subsubsection{CP-ENDOR experiments}

The experimental CP-ENDOR spectra (Fig. 5.5) were obtained selectively on EPR $\alpha$ (B), $\beta(\mathrm{C})$ and $\gamma(\mathrm{D})$ using the standard CP-ENDOR sequence (A). After the selective $\pi / 2$ preparation pulse of $t_{\pi / 2, \mathrm{mw}}=200 \mathrm{~ns}$ duration, a $150 \mu \mathrm{s}$ lasting spinlock pulse at a fixed rf offset value was set. The ENDOR information is then read-out from a rf inversion pulse followed by a selective Hahn-echo detection sequence. The rf during spinlock was set according to the values obtained from the eNCP studies.

Thus, on EPR $\alpha$ it was set to $\Delta \omega_{\mathrm{n}}^{\mathrm{CP}} / 2 \pi=3.9 \mathrm{MHz}\left(\mathrm{CP}\left[5^{\prime}-6^{\prime}\right]\right)$ and $-3.8 \mathrm{MHz}\left(\mathrm{CP}\left[2^{\prime}-3^{\prime}\right]\right)$. Additionally, the ENDOR spectrum shown at the top of Fig. 5.5, B was recorded with an rf offset of $\Delta \omega_{\mathrm{n}}^{\mathrm{CP}} / 2 \pi=-5.1 \mathrm{MHz}\left(\mathrm{CP}\left[2^{\prime}-6^{\prime}\right]\right)$ during the spinlock pulse. This value does not fulfill a matching condition for the excited EPR $\alpha$ transition (see Table 5.3). Thus, two almost equally intense ENDOR transitions separated by $A / 2 \pi$ are observed. At CP-matched rf offsets, one peak with positively enhanced and one with vanishing signal intensity is obtained. The experiments on $\operatorname{EPR} \beta$ and $\gamma$ were performed in the same manner.

At EPR $\beta$ rf offsets of $\Delta \omega_{\mathrm{n}}^{\mathrm{CP}} / 2 \pi=-5.1,-3.8,+3.9,+5.2 \mathrm{MHz}$ for effective $\mathrm{CP}$ and $\Delta \omega_{\mathrm{n}}^{\mathrm{CP}} / 2 \pi=-2.3 \mathrm{MHz}$ without $\mathrm{CP}$ matching were chosen. The non-matched CP-ENDOR spectrum (top of $\mathrm{C}$ ) reveals four peaks with slightly higher intensity of the high frequency peaks. The large splitting was assigned to hyperfine coupling and the small splitting to quadrupole coupling $(=3 Q)$. Further, at CP-matched rf offsets asymmetric CP-ENDOR spectra were obtained. In all CP-matched experiments, two out of the four NMR transitions were enhanced, one vanishes and one gets reduced compared to the corresponding non-matched CP-ENDOR intensity.

Finally, CP-ENDOR on EPR $\gamma$ with rf offets of $\Delta \omega_{\mathrm{n}}^{\mathrm{CP}} / 2 \pi=-5.1,+5.2 \mathrm{MHz}$ for matched and $\Delta \omega_{\mathrm{n}}^{\mathrm{CP}} / 2 \pi=-3.9 \mathrm{MHz}$ for non-matched CP-ENDOR were recorded (D). Again, the non-matched CP-ENDOR spectrum (top of D) reveals two peaks separated by $A$ with slightly higher intensity of the high frequency peaks and the CP-ENDOR spectra show asymmetric peaks. Thus, we obtain CP-ENDOR characteristic asymmetric spectra when satisfying a matching condition of the excited EPR line and symmetric spectra for non-matched CP-ENDOR experiments. Further, the asymmetry pattern gets interchanged when changing from negative to positive offset values. 
A) standard CP-ENDOR sequence

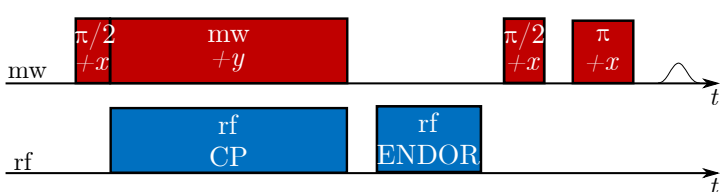

C) CP-ENDOR on EPR $\beta$
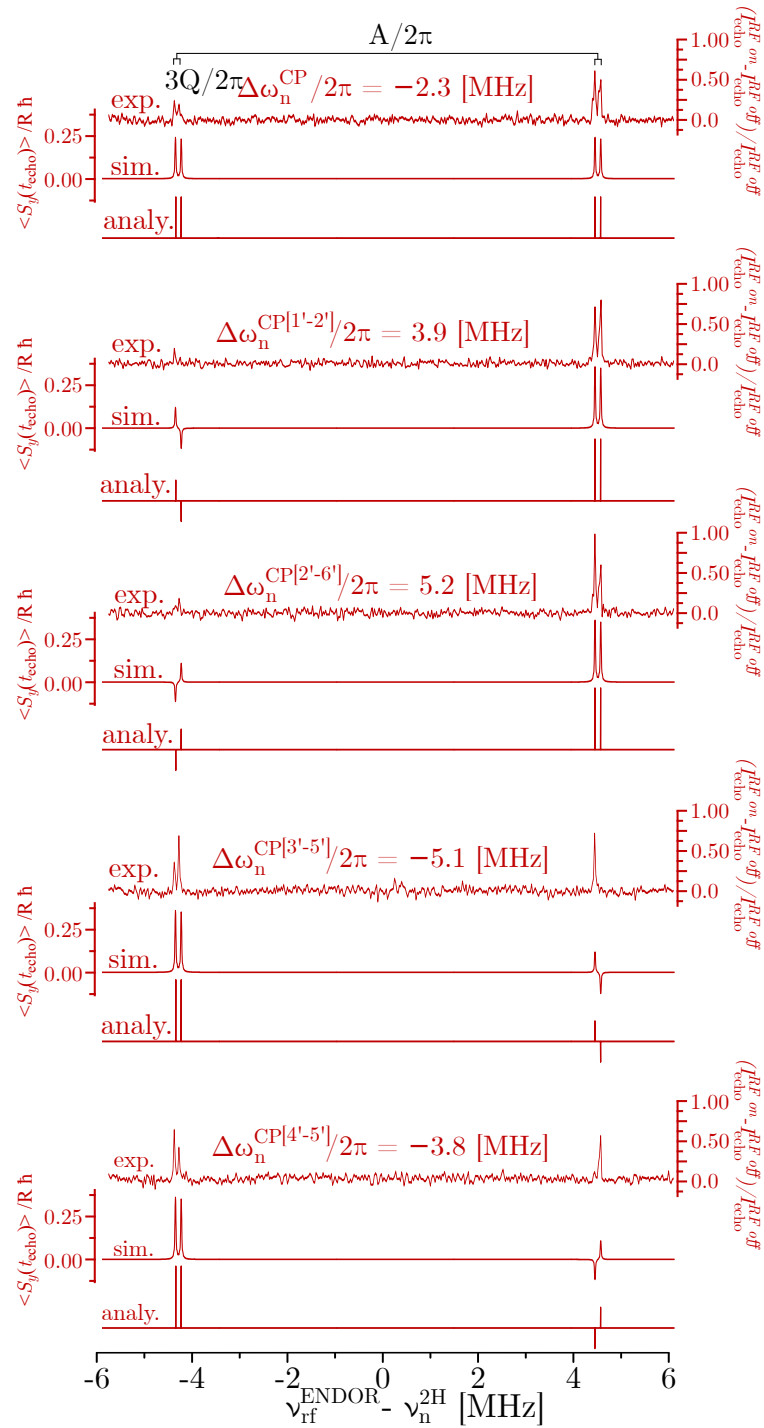

\section{B) CP-ENDOR on EPR $\alpha$}
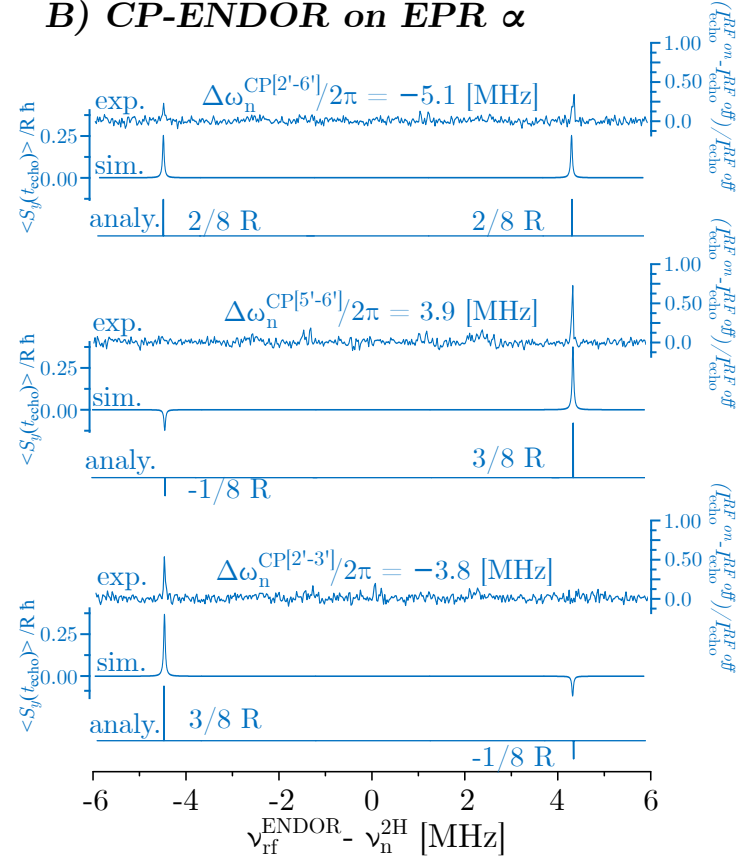

D) CP-ENDOR on EPR $\gamma$
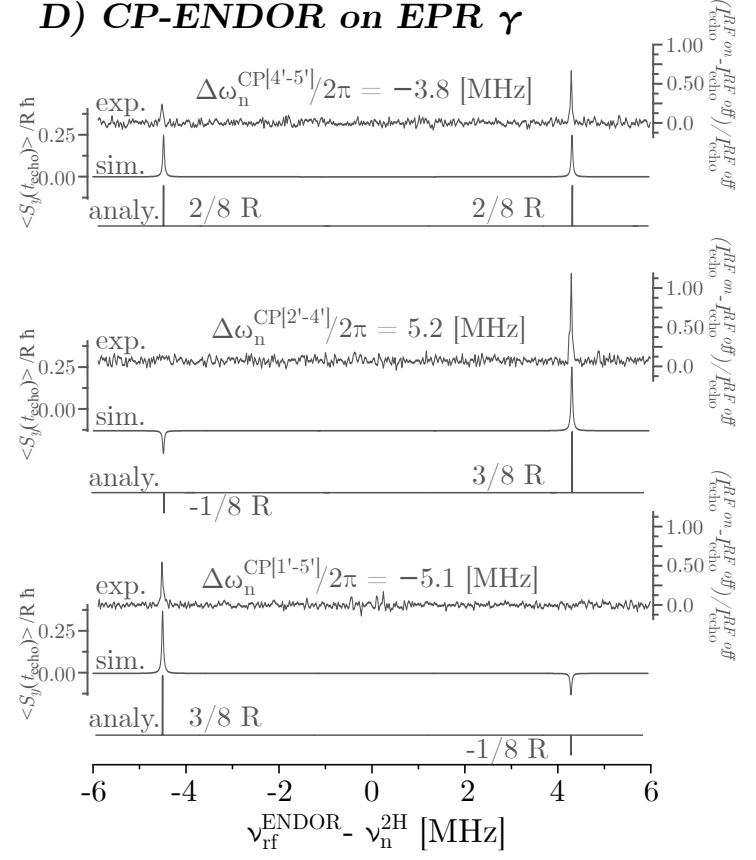

Figure 5.5: ${ }^{2} \mathrm{H}$ CP-ENDOR on a deuterated malonic acid single crystal. Experimental, analytical and numerical results are shown for excitation on each EPR manifold. A) Schematic standard CP-ENDOR sequence. B) CP-ENDOR results on EPR $\alpha$ for two CP-effective irradiations (middle and bottom) and one non-effective irradiation (top). $C$ ) CP-ENDOR results on EPR $\beta$ at one non-effective CP irradiation (top) and four CP-effective rf irradiations. D) CP-ENDOR results on EPR $\gamma$ for two CP-effective rf irradiations (middle and bottom) and one non-effective irradiation (top). CP characteristic asymmetric ENDOR spectra are obtained for the CP-effective irradiation and symmetric ENDOR peaks for non-effective irradiation. Exp. conditions: $T=40 \mathrm{~K}, \nu_{\mathrm{EPR}}=94 \mathrm{GHz}$, shot repetition time $=100 \mathrm{~ms} ; t_{\pi / 2, \mathrm{mw}}=200 \mathrm{~ns}$, $\omega_{1 \mathrm{e}} / 2 \pi=1.25 \mathrm{MHz}, \omega_{1 \mathrm{n}} / 2 \pi=16 \mathrm{kHz}, t_{\mathrm{CP}}=150 \mu \mathrm{s}, t_{\pi, \mathrm{rf}}=28 \mu \mathrm{s}, 1 \mathrm{shot} /$ point, random rf acquisition, 100 scans. Sim. parameters: $A / 2 \pi=-8.9 \mathrm{MHz}, Q / 2 \pi=-0.04 \mathrm{MHz}$, $\omega_{1 \mathrm{e}} / 2 \pi=1.18 \mathrm{MHz}, \omega_{1 \mathrm{n}} / 2 \pi=13 \mathrm{kHz}, \Delta \omega_{\mathrm{e}}= \pm A / 2 \pi, 0$. 


\subsubsection{CP-ENDOR intensities from analytical treatment}

In the following analysis, the density operator formalism is used to predict the CPENDOR intensities. The density matrix evolution during the pulse sequence under relaxation is evaluated for each of the eight $\mathrm{CP}$ matching conditions and finally the ENDOR effect calculated. However, we do not consider relaxation in terms of precise electron and nuclear spin-lattice relaxation times $T_{1 \mathrm{e}, 1 \mathrm{n}}$, spin-spin relaxation times $T_{2 \mathrm{e}, 2 \mathrm{n}}$ or as an electron spin rotating frame relaxation time $T_{1 \rho, \mathrm{e}}$ during the $\mathrm{CP}$ step, but consider the relaxation related processes in a phenomenological fashion.

The density matrix (see Subsec. 5.2.2) at thermal equilibrium assuming Boltzmann distribution 29$]$ is defined as

$$
\rho_{\mathrm{B}}=\frac{\exp (-\beta \hbar \tilde{H})}{\operatorname{Tr}\{\exp (-\beta \hbar \tilde{H})\}}
$$

with $\beta=1 / k_{\mathrm{B}} T$ and $\tilde{H}$ as defined in the previous section. The expression simplifies under high temperature approximation $\hbar \omega_{\mathrm{e}} \ll k_{\mathrm{B}} T$ (HTA) to

$$
\rho_{B} \simeq \frac{\mathbb{1}-\beta \hbar \tilde{H}}{\operatorname{Tr}\{\mathbb{1}\}}
$$

with $\operatorname{Tr}\{\mathbb{1}\}=6$ for the $S=1 / 2, I=1$ spin system, such that

$$
\rho_{\mathrm{B}}=\frac{\mathbb{1}}{6}-\frac{1}{6} \beta \hbar \tilde{H}
$$

The traceless part of $\rho_{\mathrm{B}}$ is

$$
\begin{aligned}
\rho_{0} & =-\frac{1}{6} \frac{\hbar \gamma_{e} B_{0}}{k_{\mathrm{B}} T} \tilde{S}_{z} \\
& =-R \tilde{S}_{z}
\end{aligned}
$$

which we takes the explicit form

$$
\rho_{0}=-R \tilde{S}_{z}=-R\left[\begin{array}{cccccc}
1 / 2 & 0 & 0 & 0 & 0 & 0 \\
0 & 1 / 2 & 0 & 0 & 0 & 0 \\
0 & 0 & 1 / 2 & 0 & 0 & 0 \\
0 & 0 & 0 & -1 / 2 & 0 & 0 \\
0 & 0 & 0 & 0 & -1 / 2 & 0 \\
0 & 0 & 0 & 0 & 0 & -1 / 2
\end{array}\right]
$$


In the following, the diagonal elements of $\rho$ are written in a reduced notation with double square brackets [[ ]] in a column:

$$
\left.\rho_{0}=-R \tilde{S}_{z}=-R\left[\left[\begin{array}{c}
1 / 2 \\
1 / 2 \\
1 / 2 \\
-1 / 2 \\
-1 / 2 \\
-1 / 2
\end{array}\right]\right]=\left[\begin{array}{c}
\rho_{11} \\
\rho_{22} \\
\rho_{33} \\
\rho_{44} \\
\rho_{55} \\
\rho_{66}
\end{array}\right]\right]
$$

This is defined as the initial density matrix at the beginning of the treatment $\rho\left(t_{0}\right)=\rho_{0}$.

Consequently,

$$
\left.\left.\left\langle\tilde{S}_{z}\left(t_{0}\right)\right\rangle=\hbar \operatorname{Tr}\left\{\rho(t=0) \tilde{S}_{z}\right\}=\hbar \operatorname{Tr}\left\{\left[\begin{array}{c}
-R / 2 \\
-R / 2 \\
-R / 2 \\
R / 2 \\
R / 2 \\
R / 2
\end{array}\right]\right] \cdot\left[\begin{array}{c}
1 / 2 \\
1 / 2 \\
1 / 2 \\
-1 / 2 \\
-1 / 2 \\
-1 / 2
\end{array}\right]\right)\right\}=-1.5 R \hbar
$$

Note that the negative sign reflects the fact that $\left\langle\tilde{S}_{z}\left(t_{0}\right)\right\rangle$ is oriented oppositely to its magnetic moment due to the negative gyromagnetic ratio and subsequently the magnetic moment results positive. For the following calculations $1 / 3\left\langle\tilde{S}_{z}\left(t_{0}\right)\right\rangle \equiv-R \hbar / 2$ in HTA and thermal equilibrium is considered. When EPR excitation occurs for example selectively on the EPR $\alpha$ transition $([3-6])$ by means of a $\left(\frac{\pi}{2}\right)_{x}$ mw pulse the initial density matrix $\rho\left(t_{0}\right)$ in the doubly rotating frame becomes $\rho\left(0^{+}\right)$

$$
\begin{aligned}
\rho\left(0^{+}\right) & =e^{-i \frac{\pi}{2} I_{x}^{3-6}}\left\{-R\left(I_{z}^{1-4}+I_{z}^{2-5}+I_{z}^{3-6}\right)\right\} e^{i \frac{\pi}{2} I_{x}^{3-6}} \\
& =-R I_{z}^{1-4}-R I_{z}^{2-5}-R \cos \left(\frac{\pi}{2}\right) I_{z}^{3-6}+R \sin \left(\frac{\pi}{2}\right) I_{y}^{3-6} \\
& =-R\left(I_{z}^{1-4}+I_{z}^{2-5}-I_{y}^{3-6}\right) .
\end{aligned}
$$

In order to understand the spin dynamics during CP, it is convenient to transform $\rho\left(0^{+}\right)$ into the tilted frame of the $\alpha$ manifold. The transformation into the tilted frame is performed similar to Eq. 5.23 via $U_{x} \rho U_{x}^{-1}$ with $U_{x}$ as defined in Eq.5.2.2. The rotation 
angles for the malonic acid case are $\theta_{\alpha}=\pi / 2, \theta_{\beta}=\theta_{\gamma}=0$ according to Table 5.2. The transformation results in

$$
\begin{aligned}
\rho^{\prime}\left(0^{+}\right) & =U_{x}\left(\theta_{\gamma}, \theta_{\beta}, \theta_{\alpha}\right)\left\{\rho\left(0^{+}\right)\right\} U_{x}^{-1}\left(\theta_{\gamma}, \theta_{\beta}, \theta_{\alpha}\right) \\
& =U_{x}(0,0, \pi / 2)\left\{-R\left(I_{z}^{1-4}+I_{z}^{2-5}-I_{y}^{3-6}\right)\right\} U_{x}^{-1}(0,0, \pi / 2) \\
& =-R I_{z}^{1-4}-R I_{z}^{2-5}+R I_{z}^{3-6} .
\end{aligned}
$$

Here, the superscript ' denotes the density matrix in the tilted frame. When no CP condition is matched or no rf field is applied, the diagonal elements of $\rho^{\prime}\left(0^{+}\right)$stay locked along the lock field and decay with a characteristic relaxation time $T_{1 \rho}$. All other matrix elements decay with a shorter relaxation time which is the dephasing time of the magnetization rotating around the lock field due to $B_{1}$ inhomogeneity and spin-spin relaxation. This leads to $\rho^{\prime}\left(t_{\text {spinlock }}\right)$, with only diagonal elements remaining:

$$
\left.\rho^{\prime}\left(t_{\text {spinlock }}\right)=-R I_{z}^{1-4}-R I_{z}^{2-5}+R I_{z}^{3-6}=\left[\begin{array}{c}
-R / 2 \\
-R / 2 \\
R / 2 \\
R / 2 \\
R / 2 \\
-R / 2
\end{array}\right]\right]
$$

If at this stage rf irradiation is applied during the spinlock period at rf offset corresponding to an effective matching condition, the previously described off-diagonal elements of the tilted Hamiltonian $\tilde{\mathcal{H}}^{\prime}$ drive transitions between the corresponding degenerate energy levels. For example the matching conditions $\left[2^{\prime}-3^{\prime}\right]$ and $\left[5^{\prime}-6^{\prime}\right]$ at EPR $\alpha$ (see Table 5.3) result in:

$$
\rho^{\prime}\left(t_{\text {spinlock }}\right)=\left[\left[\begin{array}{c}
-R / 2 \\
-R / 2 \\
R / 2 \\
R / 2 \\
R / 2 \\
-R / 2
\end{array}\right] \rightarrow \rho^{\prime}\left(t_{\mathrm{CP}}^{\left[2^{\prime}-3^{\prime}\right]}\right)=\left[\left[\begin{array}{c}
-R / 2 \\
\frac{-R / 2+R / 2}{2} \\
\frac{+R / 2-R / 2}{2} \\
R / 2 \\
R / 2 \\
-R / 2
\end{array}\right]\right]=\left[\begin{array}{c}
-R / 2 \\
0 \\
0 \\
R / 2 \\
R / 2 \\
-R / 2
\end{array}\right]\right]
$$


and

$$
\left.\left.\rho^{\prime}\left(t_{\text {spinlock }}\right)=\left[\left[\begin{array}{c}
-R / 2 \\
-R / 2 \\
R / 2 \\
R / 2 \\
R / 2 \\
-R / 2
\end{array}\right]\right] \rightarrow \rho^{\prime}\left(t_{\mathrm{CP}}^{\left[5^{\prime}-6^{\prime}\right]}\right)=\left[\begin{array}{c}
-R / 2 \\
-R / 2 \\
R / 2 \\
R / 2 \\
\frac{+R / 2-R / 2}{2} \\
\frac{-R / 2+R / 2}{2}
\end{array}\right]\right]=\left[\begin{array}{c}
-R / 2 \\
-R / 2 \\
R / 2 \\
R / 2 \\
0 \\
0
\end{array}\right]\right]
$$

Whereas if in this example degeneracy is created between levels $\left[1^{\prime}-2^{\prime}\right]$ we obtain

$$
\rho^{\prime}\left(t_{\text {spinlock }}\right)=\left[\left[\begin{array}{c}
-R / 2 \\
-R / 2 \\
R / 2 \\
R / 2 \\
R / 2 \\
-R / 2
\end{array}\right] \rightarrow \rho^{\prime}\left(t_{\mathrm{CP}}^{\left[1^{\prime}-2^{\prime}\right]}\right)=\left[\left[\begin{array}{c}
\frac{-R / 2-R / 2}{2} \\
\frac{-R / 2-R / 2}{2} \\
R / 2 \\
R / 2 \\
R / 2 \\
-R / 2
\end{array}\right]\right]=\left[\begin{array}{c}
-R / 2 \\
-R / 2 \\
R / 2 \\
R / 2 \\
R / 2 \\
-R / 2
\end{array}\right]\right]
$$

and there is no net effect on the density matrix. (In Section 5.2.2 non-effective matching conditions were analytically derived. They were attribute as non-effective because they create degeneracy of levels in the tilted frame which are already equally populated. This a representative example of such cases.)

At next, the density matrix after the CP step is transferred back in the doubly rotating frame via the inverse transformation of Eq. 5.23 (solved numerically):

$$
\begin{aligned}
\rho\left(t_{\mathrm{CP}}^{\left[i^{\prime}-j^{\prime}\right]}\right) & =U_{x}^{-1}\left(\theta_{\gamma}, \theta_{\beta}, \theta_{\alpha}\right)\left\{\rho^{\prime}\left(t_{\mathrm{CP}}^{\left[i^{\prime}-j^{\prime}\right]}\right)\right\} U_{x}\left(\theta_{\gamma}, \theta_{\beta}, \theta_{\alpha}\right) \\
& =U_{x}^{-1}(0,0, \pi / 2)\left\{\rho^{\prime}\left(t_{\mathrm{CP}}^{\left[i^{\prime}-j^{\prime}\right]}\right)\right\} U_{x}(0,0, \pi / 2)
\end{aligned}
$$

and after letting the off-diagonal elements decay for time $\tau>T_{2 \mathrm{e}}$, we obtain the elements

$$
\left.\rho\left(t_{\mathrm{CP}}^{\left[2^{\prime}-3^{\prime}\right]}\right)=\left[\left[\begin{array}{c}
-R / 2 \\
0 \\
-R / 4 \\
R / 2 \\
R / 2 \\
-R / 4
\end{array}\right]\right], \rho\left(t_{\mathrm{CP}}^{\left[5^{\prime}-6^{\prime}\right]}\right)=\left[\left[\begin{array}{c}
-R / 2 \\
-R / 2 \\
R / 4 \\
R / 2 \\
0 \\
-R / 4
\end{array}\right]\right], \rho\left(t_{\mathrm{CP}}^{\left[1^{\prime}-2^{\prime}\right]}\right)=\left[\begin{array}{c}
0 \\
0 \\
R / 2 \\
R / 2 \\
R / 2 \\
R / 2
\end{array}\right]\right]
$$


We can now calculate ENDOR line intensity of the detected transition as proportional to the density matrix coefficient of $I_{z}^{1-4}, I_{z}^{2-5}$ or $I_{z}^{3-6}$ just after the selective $\pi \mathrm{rf}$ ENDOR pulse. For clearance, the schematic energy diagram with negative hyperfine and quadrupole coupling, as it is the case for malonic acid, is shown in Fig. 5.6, A.

A) Energy diagram $S=1 / 2, I=1$

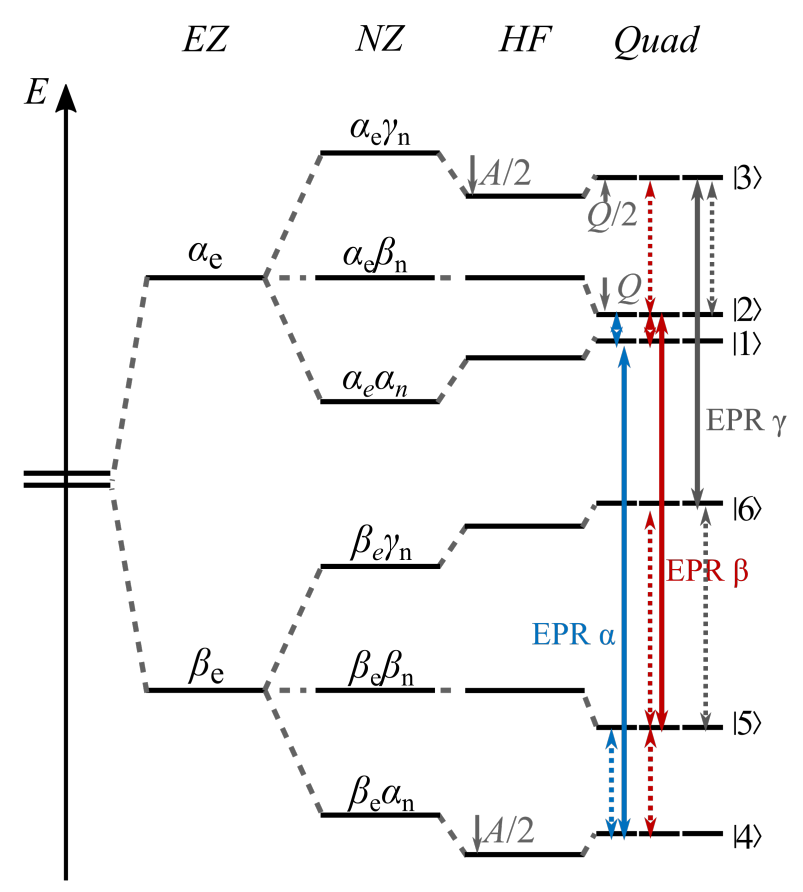

$\uparrow \uparrow \uparrow \begin{gathered}\text { EPR } \\ \text { transitions }\end{gathered}$

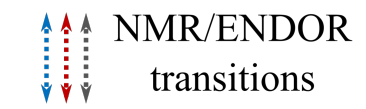

\section{B) EPR resonances}

for $A>0$

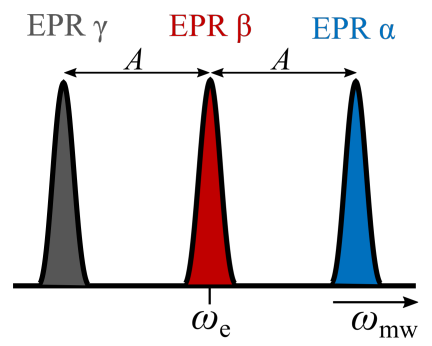

C) ENDOR resonances for $A>0$

ENDOR@EPR $\gamma$

[2-3]
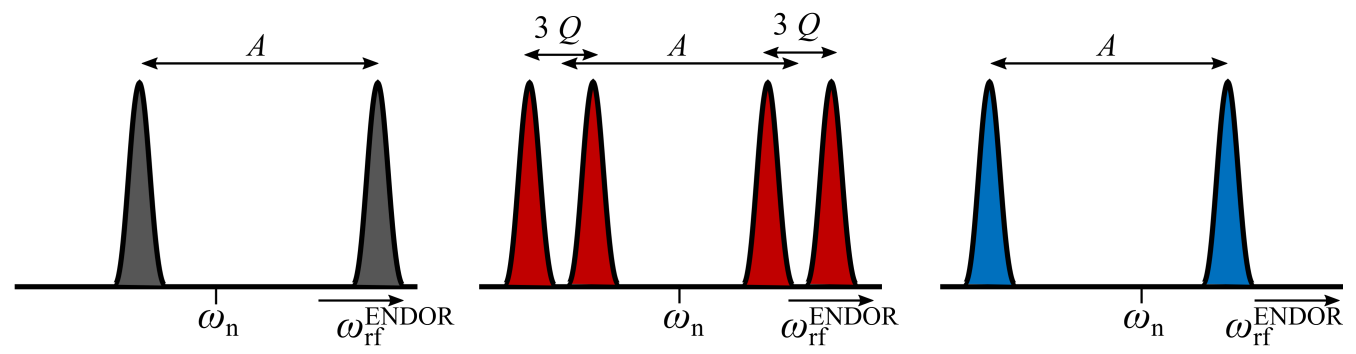

Figure 5.6: First-order energy diagram for an electron spin-1/2 coupled to a nuclear spin-1 and negative hyperfine and quadrupole couplings. $A$ ) Energy diagram for the spin system $S=1 / 2, I=1, A / 2 \pi<0, A / 2 \pi<\omega_{\mathrm{n}}$ and $Q / 2 \pi<0$. EPR transitions are assigned by solid lines, NMR/ENDOR transitions by dashed lines. B) EPR line assignment in frequency domain EPR spectrum with numbering of electron spin levels as defined in the analytical treatment. $C$ ) Assignment of ENDOR lines in the ENDOR spectrum. The number of the observed ENDOR lines relates to the number of allowed NMR transitions $\left(\Delta m_{I}= \pm 1\right)$ sharing an energy level with the respectively excited EPR transition. 
The energy levels are numbered according to our definition for the analytical treatment with all EPR transitions (solid lines) and NMR transitions (dashed lines) assigned. The corresponding EPR and ENDOR lines illustrated in Fig. 5.6, B and Fig. 5.6, C, respectively. In case of selective detection of EPR $\alpha$ ([3-6]), two NMR transitions [5-6] and [2-3] are observed in the ENDOR spectrum (see Fig. 5.6 C, blue). For the rf ENDOR pulse on-resonant with NMR transition [5-6] after CP on [2'-3'] we obtain the ENDOR signal intensity from

$$
\begin{aligned}
S_{\text {echo, } \alpha}^{\mathrm{NMR} 5-6}\left(\mathrm{CP}\left[2^{\prime}-3^{\prime}\right]\right) & \sim \operatorname{Tr}\left\{\left(U_{\pi}^{5-6}\right) \rho\left(t_{\mathrm{CP}}^{\left[2^{\prime}-3^{\prime}\right]}\right)\left(U_{\pi}^{5-6}\right)^{-1} I_{z}^{3-6}\right\} \\
& \sim \operatorname{Tr}\left\{\rho\left(t_{\mathrm{CP}}^{\left[2^{\prime}-3^{\prime}\right]}\right)\left(U_{\pi}^{5-6}\right)^{-1} I_{z}^{3-6}\left(U_{\pi}^{5-6}\right)\right\} \\
& \sim \operatorname{Tr}\left\{\rho\left(t_{\mathrm{CP}}^{\left[2^{\prime}-3^{\prime}\right]}\right) I_{z}^{3-5}\right\}
\end{aligned}
$$

and likewise for the NMR transition [2-3]

$$
\begin{aligned}
S_{\text {echo, } \alpha}^{\mathrm{NMR} 2-3}\left(\mathrm{CP}\left[2^{\prime}-3^{\prime}\right]\right) & \sim \operatorname{Tr}\left\{\left(U_{\pi}^{2-3}\right) \rho\left(t_{\mathrm{CP}}^{\left[2^{\prime}-3^{\prime}\right]}\right)\left(U_{\pi}^{2-3}\right)^{-1} I_{z}^{3-6}\right\} \\
& \sim \operatorname{Tr}\left\{\rho\left(t_{\mathrm{CP}}^{\left[2^{\prime}-3^{\prime}\right]}\right)\left(U_{\pi}^{2-3}\right)^{-1} I_{z}^{3-6}\left(U_{\pi}^{2-3}\right)\right\} \\
& \sim \operatorname{Tr}\left\{\rho\left(t_{\mathrm{CP}}^{\left[2^{\prime}-3^{\prime}\right]}\right) I_{z}^{2-6}\right\}
\end{aligned}
$$

The intensities when matching CP $\left[2^{\prime}-4^{\prime}\right]$ are calculated accordingly. CP-ENDOR intensities for all EPR transitions are summarized in Table 5.4 and the analogous calculations for EPR $\beta$ and $\gamma$ can be found in Appendix D. Note that negative signs in Table 5.4 reflect the fact that $\left\langle\tilde{S}_{z}\right\rangle$ is oriented oppositely to its magnetic moment due to the negative gyromagnetic ratio and subsequently the measured signal results positive. In summary, a CP characteristic asymmetric CP-ENDOR pattern is obtained at an effective matching condition offset (highlighted in color in Table 5.4, e.g. $-1 / 8 \mathrm{R}: 3 / 8 \mathrm{R}$ ) and symmetric ENDOR intensities of $2 / 8 \mathrm{R}: 2 / 8 \mathrm{R}$ are obtained when no $\mathrm{rf}$ is applied or the rf offset does not satisfy a matching condition. Both cases are illustrated in Fig. 5.7. 


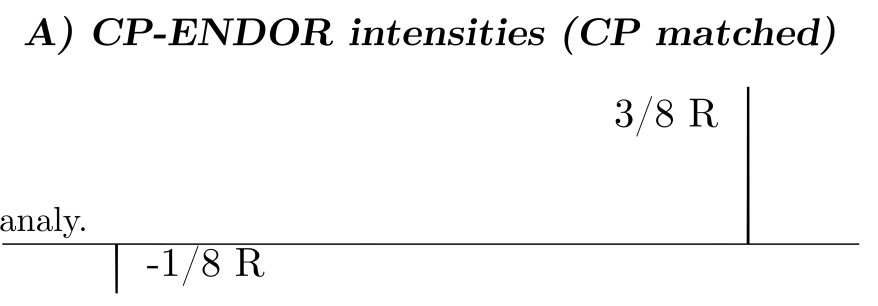

B) CP-ENDOR intensities (CP non-matched)

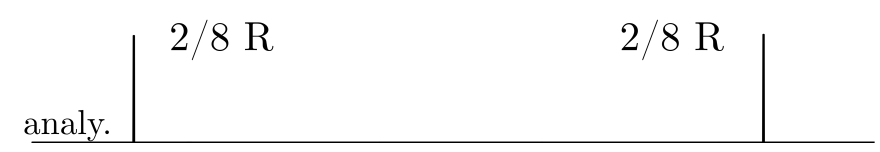

Figure 5.7: Analytically calculated CP-ENDOR intensities for CP matched and $\mathbf{C P}$ non-matched cases. A) Characteristic asymmetric CPENDOR pattern when matching a CP condition. ENDOR signal intensities are calculated to $-1 / 8 \mathrm{R}: 3 / 8 \mathrm{R}$. B) Symmetric CP-ENDOR spectra are obtained when no $\mathrm{CP}$ condition is satisfied. The ENDOR intensities are obtained to $2 / 8 \mathrm{R}: 2 / 8 \mathrm{R}$.

Table 5.4: Analytically calculated CP-ENDOR intensities for the malonic acid case. CP-ENDOR intensities calculated for the matching conditions in Table 5.3. The factor $R$ is given by Eq. 5.36.

\begin{tabular}{l||c|c|c|c|c|c|c|c}
\multicolumn{1}{c||}{} & \multicolumn{2}{c|}{ EPR $\alpha,[3-6]$} & \multicolumn{3}{c|}{ EPR $\beta,[2-5]$} & \multicolumn{2}{c}{ EPR $\gamma,[1-4]$} \\
& ENDOR & ENDOR & ENDOR & ENDOR & ENDOR & ENDOR & ENDOR & ENDOR \\
& {$[2-3]$} & {$[5-6]$} & {$[1-2]$} & {$[2-3]$} & {$[4-5]$} & {$[5-6]$} & {$[1-2]$} & {$[4-5]$} \\
\hline \hline $\mathrm{CP}\left[1^{\prime}-2^{\prime}\right]$ & $-2 / 8 \mathrm{R}$ & $-2 / 8 \mathrm{R}$ & $-3 / 8 \mathrm{R}$ & $-3 / 8 \mathrm{R}$ & $1 / 8 \mathrm{R}$ & $-1 / 8 \mathrm{R}$ & $-2 / 8 \mathrm{R}$ & $-2 / 8 \mathrm{R}$ \\
$\mathrm{CP}\left[1^{\prime}-5^{\prime}\right]$ & $-2 / 8 \mathrm{R}$ & $-2 / 8 \mathrm{R}$ & $-2 / 8 \mathrm{R}$ & $-2 / 8 \mathrm{R}$ & $-2 / 8 \mathrm{R}$ & $-2 / 8 \mathrm{R}$ & $1 / 8 \mathrm{R}$ & $-3 / 8 \mathrm{R}$ \\
$\mathrm{CP}\left[2^{\prime}-3^{\prime}\right]$ & $1 / 8 \mathrm{R}$ & $-3 / 8 \mathrm{R}$ & $-2 / 8 \mathrm{R}$ & $-2 / 8 \mathrm{R}$ & $-2 / 8 \mathrm{R}$ & $-2 / 8 \mathrm{R}$ & $-2 / 8 \mathrm{R}$ & $-2 / 8 \mathrm{R}$ \\
$\mathrm{CP}\left[2^{\prime}-4^{\prime}\right]$ & $-2 / 8 \mathrm{R}$ & $-2 / 8 \mathrm{R}$ & $-2 / 8 \mathrm{R}$ & $-2 / 8 \mathrm{R}$ & $-2 / 8 \mathrm{R}$ & $-2 / 8 \mathrm{R}$ & $-3 / 8 \mathrm{R}$ & $1 / 8 \mathrm{R}$ \\
$\mathrm{CP}\left[2^{\prime}-6^{\prime}\right]$ & $-2 / 8 \mathrm{R}$ & $-2 / 8 \mathrm{R}$ & $-3 / 8 \mathrm{R}$ & $-3 / 8 \mathrm{R}$ & $-1 / 8 \mathrm{R}$ & $1 / 8 \mathrm{R}$ & $-2 / 8 \mathrm{R}$ & $-2 / 8 \mathrm{R}$ \\
$\mathrm{CP}\left[3^{\prime}-5^{\prime}\right]$ & $-2 / 8 \mathrm{R}$ & $-2 / 8 \mathrm{R}$ & $-1 / 8 \mathrm{R}$ & $1 / 8 \mathrm{R}$ & $-3 / 8 \mathrm{R}$ & $-3 / 8 \mathrm{R}$ & $-2 / 8 \mathrm{R}$ & $-2 / 8 \mathrm{R}$ \\
$\mathrm{CP}\left[4^{\prime}-5^{\prime}\right]$ & $-2 / 8 \mathrm{R}$ & $-2 / 8 \mathrm{R}$ & $1 / 8 \mathrm{R}$ & $-1 / 8 \mathrm{R}$ & $-3 / 8 \mathrm{R}$ & $-3 / 8 \mathrm{R}$ & $-2 / 8 \mathrm{R}$ & $-2 / 8 \mathrm{R}$ \\
$\mathrm{CP}\left[5^{\prime}-6^{\prime}\right]$ & $-3 / 8 \mathrm{R}$ & $1 / 8 \mathrm{R}$ & $-2 / 8 \mathrm{R}$ & $-2 / 8 \mathrm{R}$ & $-2 / 8 \mathrm{R}$ & $-2 / 8 \mathrm{R}$ & $-2 / 8 \mathrm{R}$ & $-2 / 8 \mathrm{R}$
\end{tabular}

\subsubsection{CP-ENDOR intensities from numerical treatment}

In analogy to the numerical prediction of $\mathrm{CP}$ matching conditions also the CP-ENDOR signal intensities were calculated using the simulation strategy in presented in Subsec. 4.3.1.2. Similar to the experimental procedure, the rf frequency offsets during CP 
$\left(\Delta \omega_{\mathrm{n}}^{\mathrm{CP}}\right)$ were chosen to either satisfy or not satisfy a CP matching condition of the respective excited EPR transition. The obtained ENDOR intensities are in agreement with the analytically predicted ones (see Fig. 5.5). For example, ENDOR signal intensities of $3 / 8:-1 / 8$ at the timepoint of the echo when matching $\mathrm{CP}\left[1^{\prime}-5^{\prime}\right]$ and selective excitation of EPR $\alpha$ were observed. This is in agreement with the relative intensity ratio obtained from the analytical treatment $(3 / 8 \mathrm{R}:-1 / 8 \mathrm{R})$. For a non-matched case, equally intense ENDOR peaks are obtained. All simulated CP-ENDOR spectra are plotted in Fig. 5.5.

\subsection{Discussion of eNCP and CP-ENDOR results}

\subsection{1. ${ }^{2} \mathrm{H}$ eNCP investigations on a deuterated single crystal of malonic acid}

Overall, eNCP experiments of the deuterium were successful and we obtained strong electron spin depolarization peaks in the eNCP spectra.

In general, the obtained matching conditions can be divided in two groups. The interpretation is analogous to the previously published work from our group on a single crystal of protonated malonic acid 27 . In total eight matching conditions were identified (labeled with $\left[i^{\prime}-j^{\prime}\right]$ ) arising from interaction with the strongly coupled deuterium (highlighted in green Fig. 5.2 ) which satisfies $A / 2 \pi>\omega_{1 \mathrm{e}} / 2 \pi$ and $Q / 2 \pi \ll A / 2 \pi$. These specifically assigned matching conditions with a defined $A$ are in focus of this work because they are associated with polarization transfer to the nuclei of interest in ENDOR spectroscopy. The matching conditions for transferring polarization to such a deuterium nucleus via eNCP depend on $A / 2 \pi, Q / 2 \pi$ and $\omega_{1 \mathrm{e}} / 2 \pi$. The non-specified peaks at the center of each eNCP spectrum (at $\Delta \omega_{\mathrm{n}}^{\mathrm{CP}} / 2 \pi= \pm 1.18 \mathrm{MHz}$ and $0 \mathrm{MHz}$ ) are assigned to polarization transfer to far distant, weakly coupled nuclei in analogy to previously published work on a protonated malonic acid single crystal 27 . Possible weakly coupled nuclei could be the carboxyl deuterons and/or to deuterons in the nearby crystal unit (in the regime $A / 2 \pi<\omega_{1 \mathrm{e}} / 2 \pi$ ). Usually they are so-called matrix peaks, composed of several randomly distributed nuclei with small, almost zero hfes.

The analytical and numerical eNCP treatments are able to reproduce the matching conditions for the single crystal in good agreement with the experimental results. Additionally, the energy levels that are interchanging populations during $\mathrm{CP}\left[i^{\prime}-j^{\prime}\right]$ in the 
tilted frame were identified from the analytical treatment.

For the proton CP-ENDOR case it was reported 25$]$ that $\omega_{1 \mathrm{n}} / 2 \pi$ usually is much smaller than the detected hfc $A / 2 \pi$, which allows for the assumption of the nuclear spins quantized along the $z$-axis of the rotating frame (bare state). This is a fundamental assumption for the analytical treatment. An alternative treatment would have been to diagonalize both electron spin and nuclear part of the Hamiltonian ${ }^{[58}$. Indeed, the numerical simulation routine diagonalizes both, thus calculating a so-called doubly tilted frame. In the end, the results from both of our theoretical approaches are in very good agreement independently on which frame (tilted frame or doubly tilted frame) was considered. This agreement of the two approaches further validates that ithe diagonalization of only the electronic part in the analytical treatment is a valid assumption and the nuclear part has negligible contribution to the effective field during CP (electron spin in the dressed state, nuclear spin in the bare state).

Further, the simulation routine is free from electron- or nuclear-spin relaxation considerations. However, the averaging effect of $B_{1}$ inhomogeneity during the CP step as well as transverse relaxation during the CP mw irradiation are emulated (see Subsec. 4.3.1.4). It implies that every component vanishes which is not locked along the effective field 25 . This step is not affecting the locked component but it is crucial to eliminate the contributions which are off-resonant to the effective field. We found this step to be crucial, particularly for the later WOP CP-ENDOR simulations.

\subsection{2. ${ }^{2} \mathrm{H}$ CP-ENDOR investigations on a deuterated single crystal of malonic acid}

CP-ENDOR characteristic asymmetric as well as symmetric non-matched CP-ENDOR spectra are obtained from experimental and theoretical studies. In particular, the simulation routine was found to be robust and offers fast and reliable access to matching conditions and CP-ENDOR intensities.

Both theoretical approaches predict equally intense ENDOR lines at no CP matching. The intensities are the result of the $\pi / 2$ preparation pulse and the long microwave spinlock pulse acting together as ENDOR preparation step. Indeed, the obtained symmetric ENDOR pattern is a result of selectively turning the magnetization of one electron spin manifold out of equilibrium as illustrated in Fig. 2.13. In previous publications of proton CP-ENDOR experiments, this signal was called residual ENDOR 2 . Here, it 
will be called spinlock ENDOR, because it is associated with the long mw pulse acting as an ENDOR preparation pulse at a non-matched rf offset. In the following, the case where the rf offset satisfies a matching condition, but CP transfer is incomplete due to for instance too short contact time will be called residual ENDOR.

The CP-ENDOR polarization transfer scheme on basis of the results obtained in this thesis is illustrated in Fig. 5.8 for one of the possible CP-ENDOR pathways.

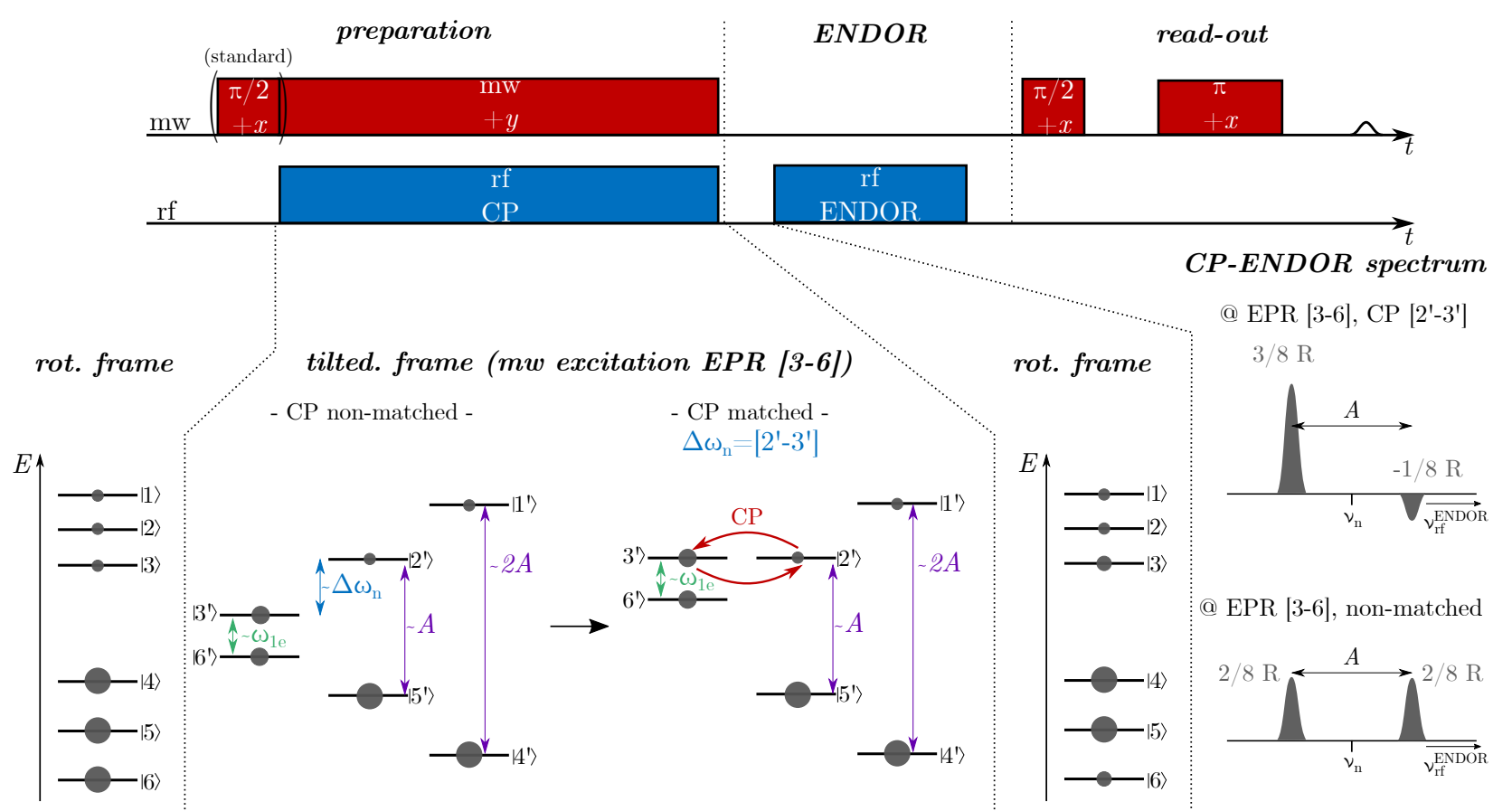

Figure 5.8: CP-ENDOR population transfer scheme for an electron spin coupled to a deuterium . Illustration for a $S=1 / 2, I=1 / 2, A<0$ spin system and $\omega_{1 \mathrm{e}} \leqslant A$. The case of selective excitation of the EPR transition $\alpha,[3-6]$ is shown. Grey balls of different size represent population of a level. The energy level splitting in the tilted frame is given by $A, \omega_{1 \mathrm{e}}$ and $\Delta \omega_{\mathrm{n}}$. CP polarization transfer is illustrated for matching $\mathrm{CP}\left[2^{\prime}-3^{\prime}\right]$. The resulting CP-ENDOR spectrum shows asymmetric lines while a non-mached CP-ENDOR experiment results in symmetric ENDOR lines.

Although predicted with equal intensities, the experimental intensities at the highfrequency side appear slightly higher. This is not only observed in CP-ENDOR, but also in eNCP and control Davies ENDOR experiments (see Fig. A1) and has been reported in previously published ENDOR studies 88 . Without giving a detailed analysis, this asymmetry might be due to non-linearity of the RF excitation and/or deviation from HFA, i.e. a so-called hf enhancement 95 . Indeed, an asymmetry of the ENDOR lines could be reproduced in a simulation of the Davies-ENDOR spectra using the software Easyspin [96] when considering hf-enhancement and complete Hamiltonian diagonalization (data not shown). This effect could not be reproduced by our numerical 
simulations, which assume high-field conditions. However, it compromises a direct quantification of the experimentally obtained relative intensities, particularly in the CP-ENDOR experiments.

Nontheless, the experimental trend of oppositely polarized NMR transitions due to CP transfer is in agreement with the theoretical predictions. The predicted change of the asymmetry pattern when changing from negative to positive rf offsets or vice versa has been observed, proving that during CP nuclear spins coupled to the electron spin with hyperfine coupling $A$ are selectively polarized. Moreover, the asymmetry factor obtained for the deuterium (when neglecting the quadrupole effect) is similar to the ratio reported for proton CP-ENDOR experiments $(3:-1) \stackrel{27}{27}$. This indicates that once two levels become degenerate for sufficiently long time, the underlying CP efficiency and dynamics are equal for both types of target nuclei.

However, in all CP-ENDOR spectra (Fig. 5.5) only positive peaks are obtained, although negative peaks are predicted from both theoretical approaches. The lack of negative peak intensities can be due to the crystal impurities that are obvious already in the ESE-detected field sweep spectrum (Fig. 5.2). The nature of such impurities remains unclear (split off the crystal, twin crystal, etc.), but all the prepared samples showed a different extend of such crystal impurity. 



\section{Without preparation pulse (WOP) CP-ENDOR spectroscopy}

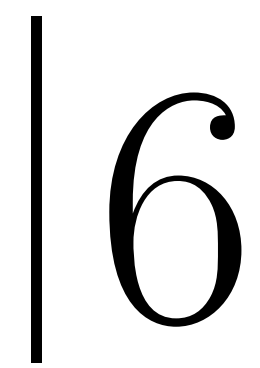

In this chapter the modified CP-ENDOR sequence called "without preparation pulse CP-ENDOR" (WOP CP-ENDOR) will be introduced. The sequence provides improved performance for the detection of small hyperfine couplings compared to the previously published CP-ENDOR and also to standard ENDOR sequences. This will be demonstrated by experiments and simulations on the standard organic radical $\alpha, \gamma,-$ bisdiphenylene- $\beta$-phenylallyl (BDPA). It will be shown that the observed enhancement over the standard CP-ENDOR sequence is the result of different hole functions of the two sequences. Furthermore, an analysis of the central hole function for standard and WOP CP-ENDOR will be presented.

\subsection{Experiments and simulations on ${ }^{2} \mathrm{H}-\mathrm{BDPA}$}

The hyperfine interaction between an electron spin and deuterium nuclei in biological samples usually is on the order of a few $\mathrm{MHz}(<5 \mathrm{MHz})$ due the low gyromagnetic ratio of the deuterium $\left(\frac{\gamma_{1 \mathrm{H}}}{\gamma_{2 \mathrm{H}}} \approx 6.5\right)$. Davies ENDOR and Mims ENDOR suffer from suppression of small hfcs or line shape distortions for the detection of small hfcs. Further, the standard CP-ENDOR sequence revealed sensitivity issues when targeting small hyperfine couplings. Because the suppression of small hfcs in Davies ENDOR is closely related to the preparation pulse conditions and the resulting excitation profile (see Sec. 2.3.2) the influence of the pulses involved in the CP step of the CP-ENDOR sequence with respect to the optimal parameters for the detection of small hfcs were examined. In the framework of these studies, the modification in which the $\pi / 2$-pulse of the CP-ENDOR preparation step was omitted resulted in significantly improved CP-ENDOR intensities for small hfcs. The modification was called without preparation (WOP) CP-ENDOR sequence (see Fig. 6.1). First, the WOP CP-ENDOR performance 
A) standard CP-ENDOR sequence

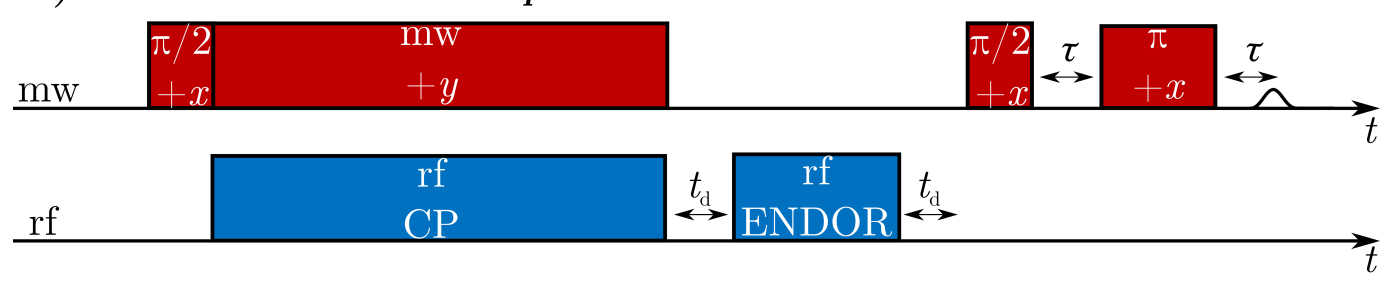

B) WOP CP-ENDOR sequence

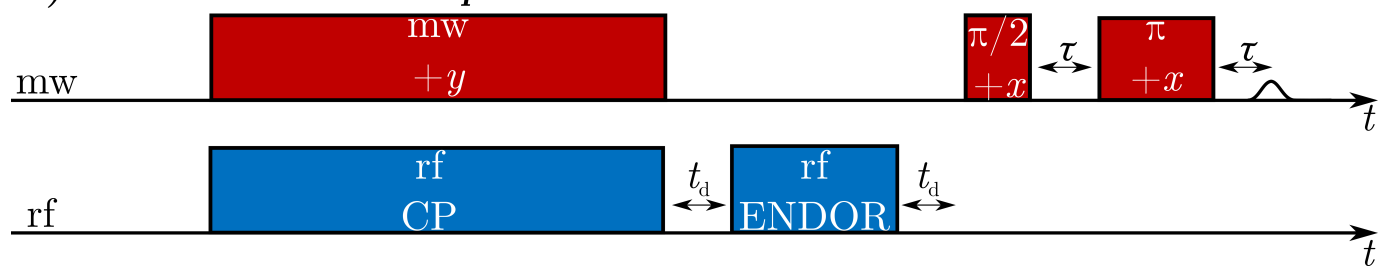

Figure 6.1: Illustration of the standard and WOP CP-ENDOR sequences. The standard CP-ENDOR pulse sequence is shown in (A) and the without preparation pulse (WOP) CP-ENDOR sequence in (B). In case of WOP CP-ENDOR, the $\pi / 2$ preparation pulse is omitted. Otherwise, the sequences are identical.

was studied on a standard EPR powder sample: the organic radical BDPA with all proton positions deuterated. The sample preparation procedure can be found in Subsection 4.1.2. The molecular ${ }^{2} \mathrm{H}-\mathrm{BDPA}$ structure as well as the electron-spin echo field sweep spectrum (ESE) at $94 \mathrm{GHz} / 3.4 \mathrm{~T}$ are shown in Fig. 6.2, A. A representative comparison of ENDOR spectra on ${ }^{2} \mathrm{H}-\mathrm{BDPA}$ is shown in Fig. 6.2, B. They were recorded with EPR excitation at the maximum of the ESE intensity (asterisk in A).

Mims ENDOR with $\tau=200$ ns (blue), Davies ENDOR (green), standard CP-ENDOR (grey) and WOP CP-ENDOR (red) were recorded at $T=80 \mathrm{~K}$, with the same shot repetition time of $25 \mathrm{~ms}$, random ENDOR rf acquisition, and 1000 scans each. All experimental conditions can be found in the figure caption. The Mims $\tau$-value was chosen in order to obtain an ENDOR line shape similar to WOP CP-ENDOR. The spectra are scaled to the same noise level of the baseline. A first qualitative comparison reveals identical line shapes, but different signal-to-noise ratios ( $\mathrm{S} / \mathrm{N})$. Mims ENDOR and WOP CP-ENDOR show a similar S/N, in comparison to standard CP-ENDOR and Davies ENDOR which show about five times less S/N. All ENDOR spectra display slightly higher intensities at the $\nu_{\mathrm{n}}^{2 \mathrm{H}}+A / 2 \pi$ resonances compared to the $\nu_{\mathrm{n}}^{2 \mathrm{H}}-A / 2 \pi$ resonances. This asymmetry is most likely associated with hardware imperfection, such as a non-linear rf amplifier power, that was not observed at an earlier stage of this thesis (see experiments shown in Appendix E). 
A) Radical structure

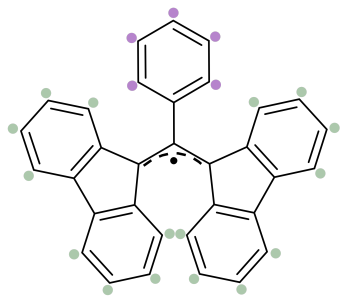

${ }^{2} \mathrm{H}-\mathrm{BDPA}$

B) ESE-detected field sweep spectrum
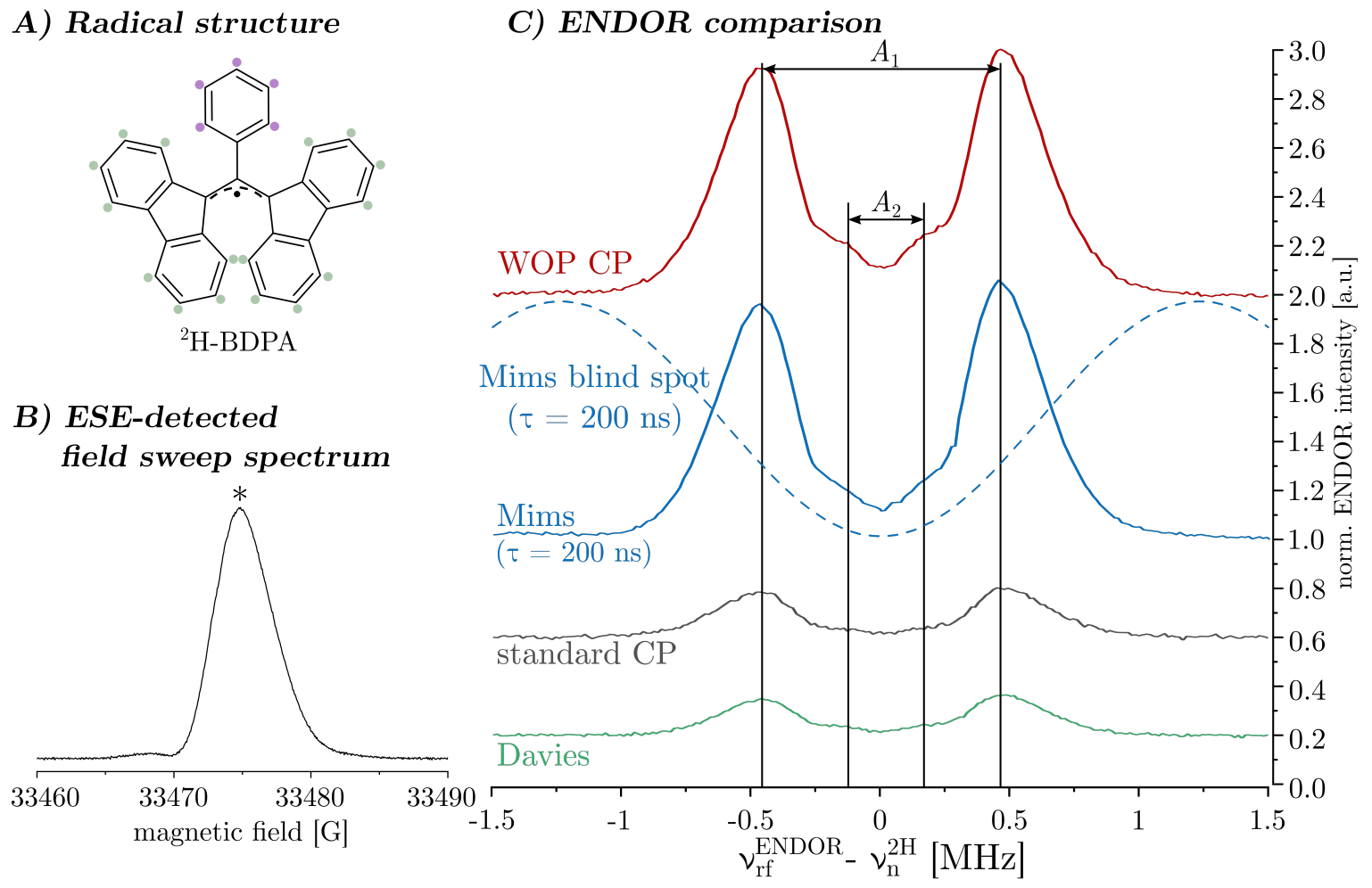

Figure 6.2: Comparison of standard CP-ENDOR, WOP CP-ENDOR, Mims ENDOR and Davies ENDOR on ${ }^{2}$ H-BDPA. A) ${ }^{2} \mathrm{H}$-BDPA radical structure with coupled deuterium nuclei highlighted in green. B) $94 \mathrm{GHz} / 3.4 \mathrm{~T}$ ESE-detected field sweep spectrum of $0.1 \%{ }^{2} \mathrm{H}$-BDPA. The asterisk marks the EPR excitaton during ENDOR acquisition. C) ENDOR comparison of standard CP-ENDOR (grey, sum of 2D dataset shown in Fig. 6.5, B), WOP CP-ENDOR (red, sum of 2D dataset shown in Fig. 6.5. A), Mims ENDOR $(\tau=200 \mathrm{~ns}$, blue, blind spot function as dashed line) and Davies ENDOR (green) recorded at maximum intensity of the field sweep spectrum (asterisk in B)). Exp. conditions: $T=80 \mathrm{~K}$, $\nu_{\mathrm{EPR}}=94 \mathrm{GHz}, t_{\pi, \mathrm{rf}}=38 \mu \mathrm{s}, \omega_{1 \mathrm{n}} / 2 \pi=13 \mathrm{kHz}$, shot repetition time $=25 \mathrm{~ms}$, 1 shot/point, random rf acquisition, 1000 scans (acquisition time $\approx 2.5 \mathrm{~h}$ ); Davies: $t_{\pi, \text { prep }}=400 \mathrm{~ns}, t_{\pi / 2, \mathrm{det}}=20 \mathrm{~ns} ;$ Mims: $t_{\pi / 2, \mathrm{mw}}=20 \mathrm{~ns} ; C P: t_{\pi / 2, \mathrm{det}}=200 \mathrm{~ns}$, $\omega_{1 \mathrm{e}} / 2 \pi=1.25 \mathrm{MHz}, t_{\mathrm{CP}}=200 \mu \mathrm{s}, \Delta \omega_{\mathrm{n}}^{\mathrm{CP}} / 2 \pi=-0.9 \mathrm{MHz}$ to $+0.9 \mathrm{MHz}$ (20 steps).

Before having a closer look at the standard CP-ENDOR and WOP CP-ENDOR performances and the corresponding simulations, some experimental setup aspects will be presented. Both CP-ENDOR experiments were performed under the same experimental conditions (temperature, averaging time, etc.) and run in a 2D fashion from an in-house PulseSPEL (BRUKER) script (Appendix F). The second dimension of this $2 \mathrm{D}$ experiment is defined by the rf matching condition/offset range $\Delta \omega_{\mathrm{n}}^{\mathrm{CP}} / 2 \pi$. Recently published proton CP-ENDOR studies showed that anisotropy effects in powder or frozen solution samples generate a broad range of matching conditions/rf offsets instead of the well-defined sharp matching conditions as observed for the single crystal 25, 27. The offset width scales with anisotropy effects as well as the applied microwave field strength 
and was identified from an eNCP experiment (see also Sec. 5.2 and Subsec. 2.4.2). The normalized eNCP spectrum of ${ }^{2} \mathrm{H}-\mathrm{BDPA}$ for $\omega_{1 \mathrm{e}} / 2 \pi=1.25 \mathrm{MHz}$ is shown in Fig. 6.3 and the corresponding matching condition range is identified as broad range from $\Delta \omega_{\mathrm{n}}^{\mathrm{CP}} / 2 \pi=-0.5$ to $+0.5 \mathrm{MHz}$.

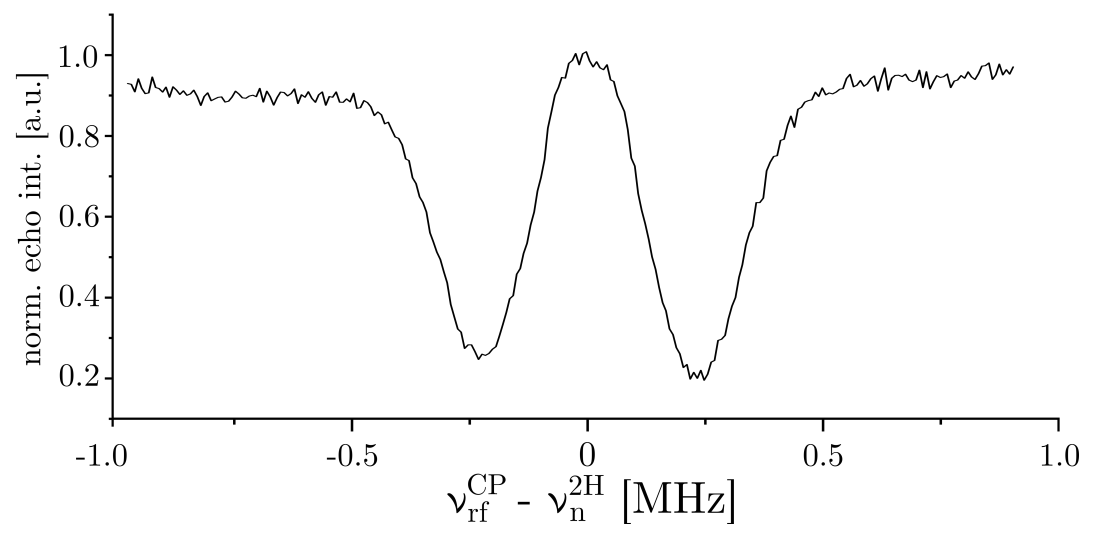

Figure 6.3: eNCP spectrum of ${ }^{2} \mathbf{H}-B D P A$. Exp. conditions: $T=80 \mathrm{~K}$, $\nu_{\mathrm{EPR}}=94 \mathrm{GHz}, \quad t_{\pi / 2, \mathrm{prep}}=200 \mathrm{~ns}, \quad t_{\pi, \text { det }}=400 \mathrm{~ns}, \omega_{1 \mathrm{e}} / 2 \pi=1.25 \mathrm{MHz}$, $\omega_{1 \mathrm{n}} / 2 \pi=13 \mathrm{kHz}, t_{\mathrm{CP}}=200 \mu \mathrm{s}$, shot repetition time $=25 \mathrm{~ms}, 1$ shot $/$ point, random rf acquisition, 300 scans (acquisition time $\approx 1 \mathrm{~h}$ ).

For the 2D CP-ENDOR experiments $20 \mathrm{rf}$ offset values (y-axis) in the determined rf range were set and 50 scans per rf offset acquired. The obtained 2D CP-ENDOR datasets are displayed as landscape and contour plots in Fig. 6.5. For direct comparison with the well-established ENDOR sequences and straight-forward analysis of hf tensor line shape, the 2D dataset is reduced to only its ENDOR dimension by summation of the 20 sub-spectra. The result is displayed in Fig. 6.2 and Fig. 6.4 together with the corresponding simulation.

In the following, the performance of the standard (grey) and WOP CP-ENDOR (red) results in Figs. 6.4 and 6.5 are discussed. The plotted simulations (dashed lines) were obtained from an extension of the single crystal simulation routine introduced in the previous chapter. For powder 2D CP-ENDOR simulations, the routine was extended to consider two further aspects: 1) a second coupled deuterium nucleus and 2) $g$-anisotropy shifts. The procedure is described in Sec. 4.3.2.1 and the simulation parameters can be found in the figure caption. Hf-tensor values are listed in Tab. 6.1. 


\section{A) Experiment}

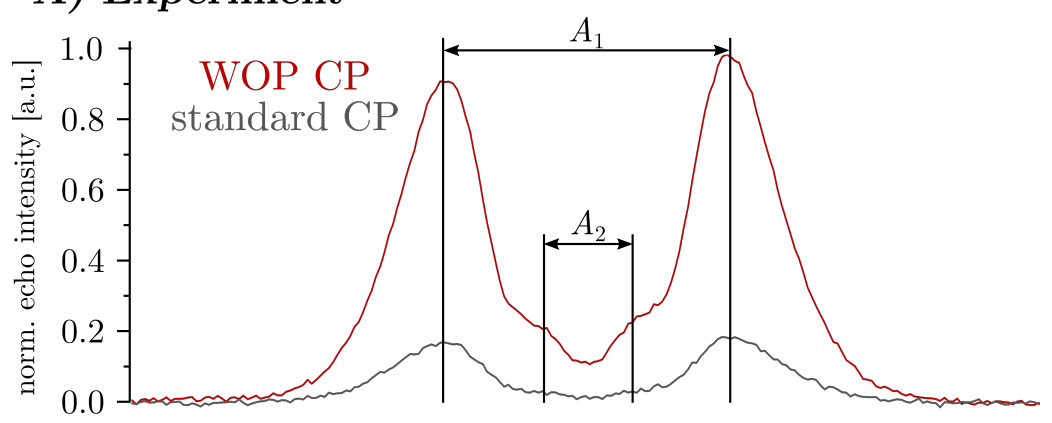

\section{B) Simulation}

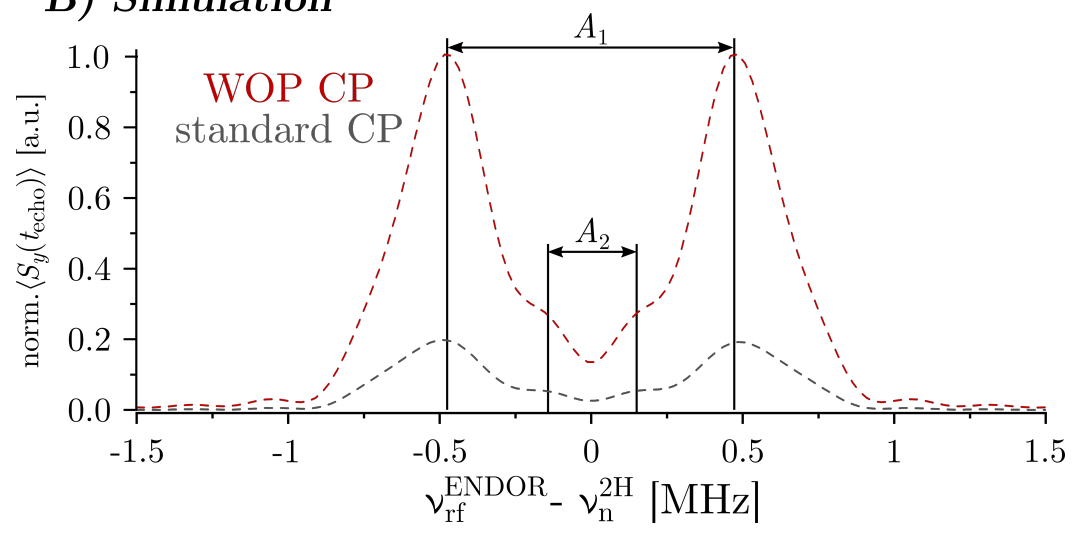

Figure 6.4: Standard CP-ENDOR and WOP CP-ENDOR experiments and simulations of ${ }^{2} \mathbf{H}$-BDPA. Summed $2 \mathrm{D}$ dataset of experiment (A) and simulation (B) of standard CP-ENDOR (grey) and WOP CP-ENDOR (red) on $0.1 \%{ }^{2} \mathrm{H}-\mathrm{BDPA}$. Exp. conditions: $T=80 \mathrm{~K}, \nu_{\mathrm{EPR}}=94 \mathrm{GHz}, t_{\pi / 2 \text {,det }}=200 \mathrm{~ns}, \omega_{1 \mathrm{e}} / 2 \pi=1.25 \mathrm{MHz}$, $\omega_{1 \mathrm{n}} / 2 \pi=13 \mathrm{kHz}, t_{\mathrm{CP}}=200 \mu \mathrm{s}, \Delta \omega_{\mathrm{n}}^{\mathrm{CP}} / 2 \pi=-0.9 \mathrm{MHz}$ to $+0.9 \mathrm{MHz}(20$ steps $)$, $t_{\pi, \mathrm{rf}}=38 \mu \mathrm{s}$, shot repetition time $=25 \mathrm{~ms}, 1 \mathrm{shot} /$ point, random rf acquisition, 1000 scans (averaging time $\approx 2.5 \mathrm{~h}$ ). Sim. parameters: $t_{\pi / 2, \mathrm{det}}=200 \mathrm{~ns}, \omega_{1 \mathrm{e}} / 2 \pi=1.0 \mathrm{MHz}$, $\omega_{1 \mathrm{n}} / 2 \pi=13 \mathrm{kHz}, t_{\mathrm{CP}}=200 \mu \mathrm{s}, \Delta \omega_{\mathrm{n}}^{\mathrm{CP}} / 2 \pi=-0.9 \mathrm{MHz}$ to $+0.9 \mathrm{MHz}$ (20 steps), $t_{\pi, \mathrm{rf}}=38 \mu \mathrm{s}, N_{\phi}=N_{\theta}=32$ (number of orientations for powder averaging).

It is noted, that the only difference between the standard CP-ENDOR simulation script and the WOP CP-ENDOR simulation script, analog to the experimental procedure, is the omitted $\pi / 2$ preparation pulse for WOP CP-ENDOR.

Table 6.1: Hyperfine tensor values for the simulation of ${ }^{2} \mathrm{H}-\mathrm{BDPA}$.

\begin{tabular}{c||c|c|c|c|c|c}
\multicolumn{1}{l||}{} & \multicolumn{3}{c|}{$\begin{array}{c}\text { Hyperfine coupling } \\
\text { obtained in this work [MHz] }\end{array}$} & \multicolumn{3}{c}{$\begin{array}{c}\text { Hyperfine coupling } \\
\text { obtained from Lit. } \frac{97]}{[\mathrm{MHz}]}\end{array}$} \\
Nuclei & $A_{x} / 2 \pi$ & $A_{y} / 2 \pi$ & $A_{z} / 2 \pi$ & $A_{x} / 2 \pi$ & $A_{y} / 2 \pi$ & $A_{z} / 2 \pi$ \\
\hline \hline $\mathrm{D}(1)$ & 0.15 & 0.25 & 0.3 & 0.25 & 0.25 & 0.27 \\
$\mathrm{D}(2)$ & 1.25 & 0.9 & 0.3 & 1.18 & 0.82 & 0.3
\end{tabular}


The 16 deuterons of the BDPA radical (green highlighted in Fig.6.2, A) show two distinguishable hfcs $A_{1}$ and $A_{2}$ in the ENDOR spectrum. Indeed, high molecular symmetry and a conjugated $\pi$-system result in two sets of nuclei with different spin densities 91 . The five nuclei at the phenyl ring (see Fig. 6.2, A, purple) are not coupled via hf-interaction to the electron spin. Finally, only two different sets of hfcs are present due to the high symmetry of the radical ${ }^{91]}$. Thus, the radical can be analyzed as a spin system with one electron spin-1/2 coupled to two nuclear spins $I_{1}$ and $I_{2}$. After summation of the experimental 2D WOP CP-ENDOR spectrum it is normalized to the maximum value and the summed $2 \mathrm{D}$ standard CP-ENDOR spectrum is scaled to the same baseline noise level. Similarly to the experimental procedure, the simulations are obtained from summation of the simulated 2D dataset. The plot show their relative $\left\langle S_{x}\left(t_{\text {echo }}\right)\right\rangle$ intensities (see also Fig. 6.1). In comparison to standard CP-ENDOR, WOP CP-ENDOR shows a factor of $\approx 5$ higher $\mathrm{S} / \mathrm{N}$ for both the experiment and the simulation.

Fig. 6.5 shows the 2D datasets for WOP CP-ENDOR (A) and standard CP-ENDOR (B). The left column displays the experimental results and the right column the simulations. WOP CP-ENDOR plots are shown with original intensities (no normalization or scaling) for both experiment and simulation. As before, the noise level of the baseline in standard CP-ENDOR is scaled to the same noise level as in WOP CP-ENDOR. Note that the color code legend refers to the contour plot only. Experiment and simulation are in good agreement in their line shape, maximum/minimum peak position and relative peak intensities. Each of these aspects is going to be examined in the following.

The line shape in the $y$-dimension $\left(\Delta \omega_{\mathrm{n}}^{\mathrm{CP}}\right)$, particularly the area of asymmetry, directly translates into the matching condition range. The characteristic CP-ENDOR asymmetry is observed prominently between $\mathrm{CP}$ rf offsets of $-0.5 \mathrm{MHz}$ and $+0.5 \mathrm{MHz}$. Maximum asymmetry is identified at $\Delta \omega_{\mathrm{n}}^{\mathrm{CP}} / 2 \pi= \pm 0.14 \mathrm{MHz}$. The range as well as the maximum peak position are in agreement with the parameters determined by the eNCP experiment (Fig. 6.3). Furthermore, every peak in the WOP CP-ENDOR spectrum shows a factor of $\approx 5$ higher intensity compared to standard CP-ENDOR. For instance, the maximum intensities in WOP CP-ENDOR are 160000 a.u. and 8 a.u. for experiment and simulation, respectively. The intensities drop by a factor of $\approx 5$ in standard CP-ENDOR to 30000 a.u. and 1.47 a.u. for experiment and simulation, respectively. In addition, the ratio between non-matched CP-ENDOR (no CP effect) and asymmetric CP-ENDOR (with CP effect) signal as well as the ratio between maximum and minimum CP effect (asymmetry ratio) are identical for both sequences. 


\section{A) $2 D W O P C P-E N D O R$ of ${ }^{2} H-B D P A$}

\section{1) Experiment}
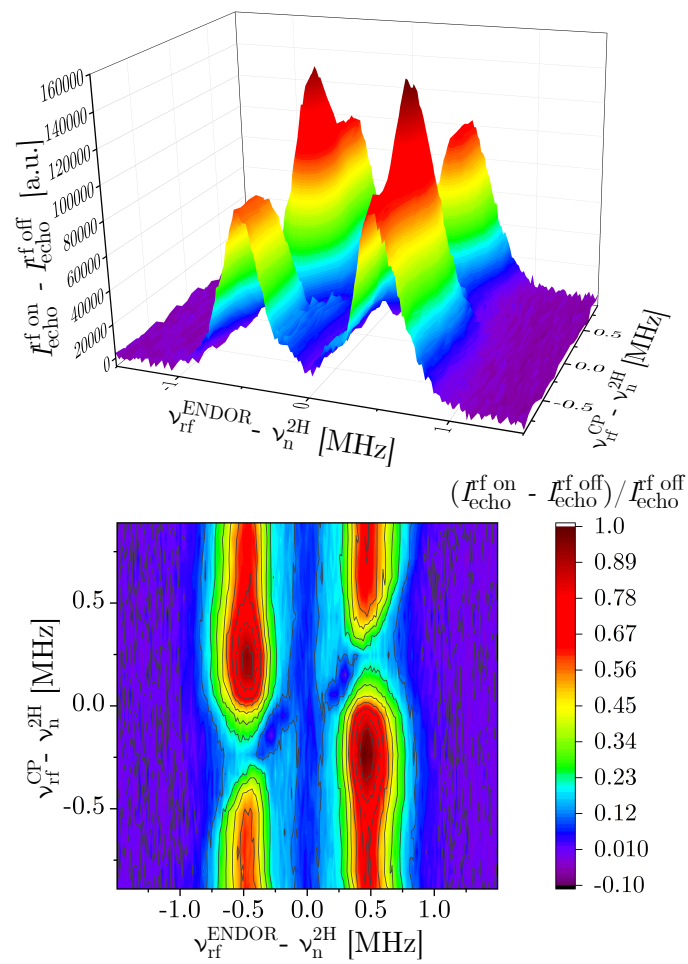

\section{2) Simulation}
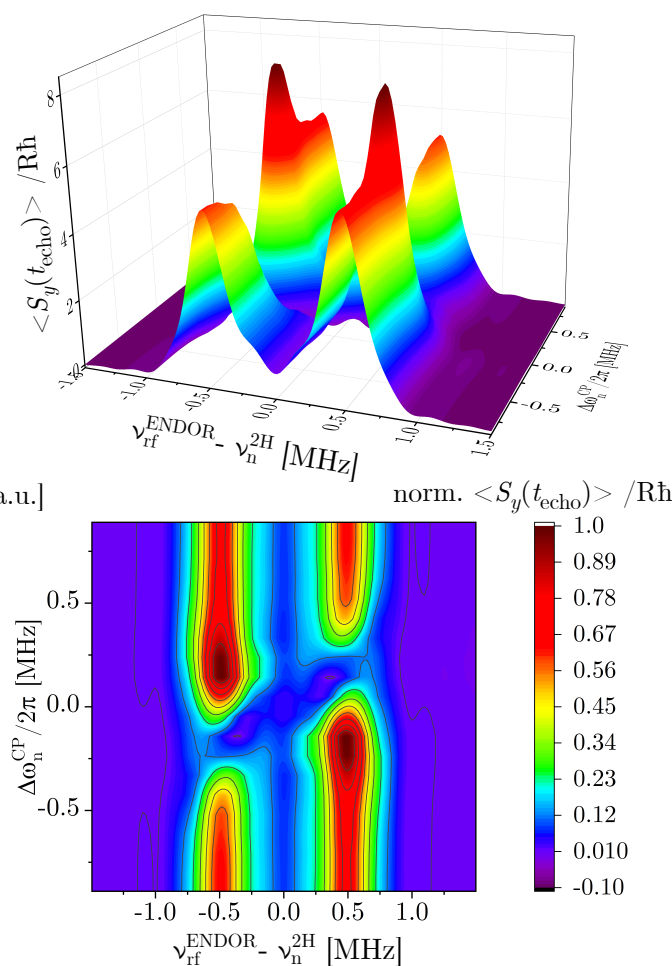

B) $2 D$ standard $C P-E N D O R$ of ${ }^{2} H-B D P A$

\section{3) Experiment}

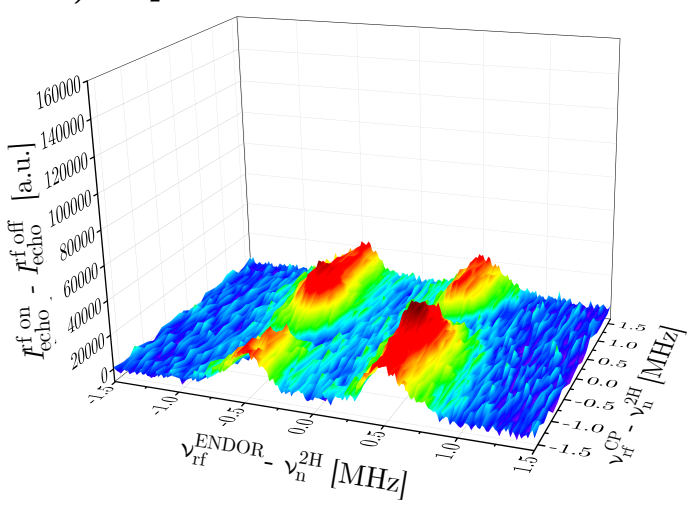

$\left(I_{\text {echo }}^{\mathrm{rf} \text { on }}-I_{\text {echo }}^{\mathrm{rf} \text { off }}\right) / I_{\text {echo }}^{\mathrm{rf} \text { off }}[$ a.u. $]$

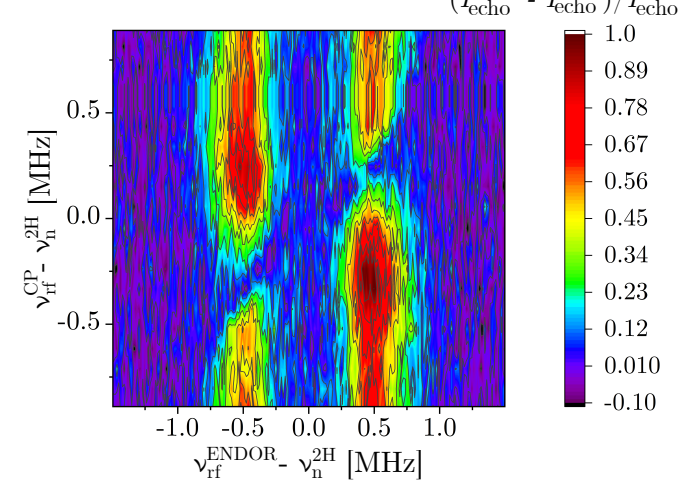

4) Simulation
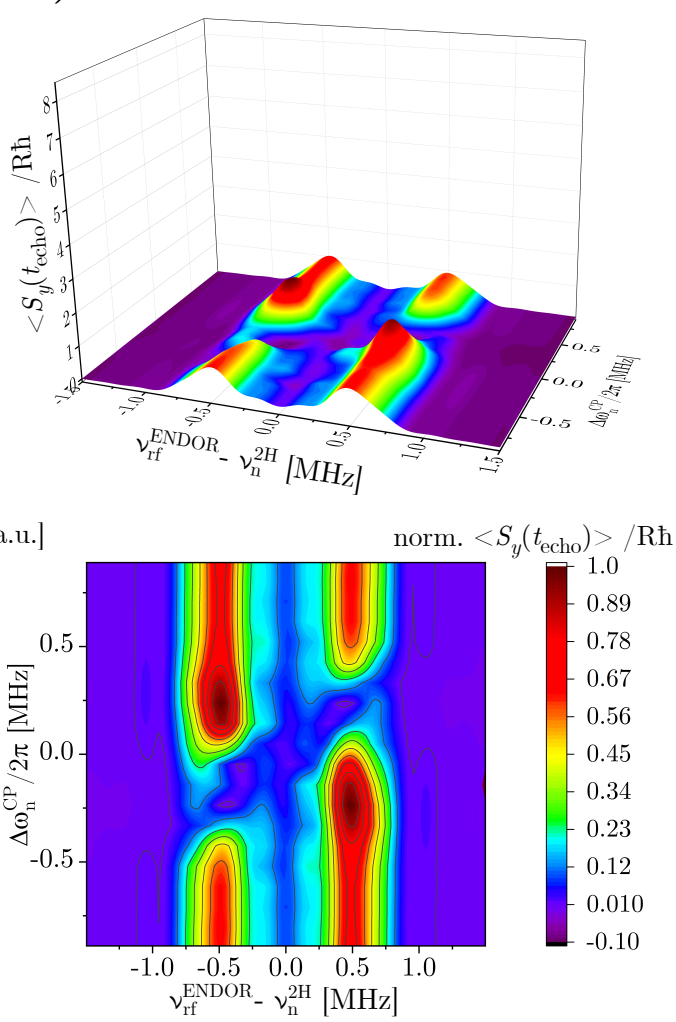

Figure 6.5: Landscape and contour plots of 2D WOP CP-ENDOR and standard 2D CP-ENDOR. A) 2D WOP CP-ENDOR spectra. B) standard CPENDOR spectra. Left) Experimental data. Right) Simulated data. Experimental conditions and simulation parameters are listed in the caption of Fig. 6.4. 
The conservation of the matching conditions as well as the identical relative intensity is demonstrated by comparing two slices from each normalized $2 \mathrm{D}$ plot shown in Fig. 6.6. It is noted that the asymmetry observed in non-matched CP-ENDOR is due to hardware imperfections and not related to $\mathrm{CP}$ asymmetry. The relative signal intensity of the non-matched CP-ENDOR signal (0.7 a.u.) to the CP-matched ENDOR signal at $\Delta \omega_{\mathrm{n}}^{\mathrm{CP}} / 2 \pi=+0.14 \mathrm{MHz}$ (1 and 0.3 a.u.) are the same for both sequences. A minimum ENDOR peak intensity is observed at $\nu_{\text {ENDOR }}-\nu_{\mathrm{n}}^{2 \mathrm{H}}=+0.28 \mathrm{MHz}$, similar for both sequences to the extend of the $\mathrm{S} / \mathrm{N}$. In a nutshell, the obtained landscapes are identical for both sequences, except for a factor of $5 \mathrm{~S} / \mathrm{N}$ improvement with WOP CP-ENDOR. The finding that the $\mathrm{CP}$ matching conditions and $\mathrm{CP}$ efficiency remain the same when omitting the $\pi / 2$ preparation pulse is further evaluated by single crystal-like CP-ENDOR simulations in Section 6.2 .

\section{A) WOP CP-ENDOR \\ B) standard CP-ENDOR}
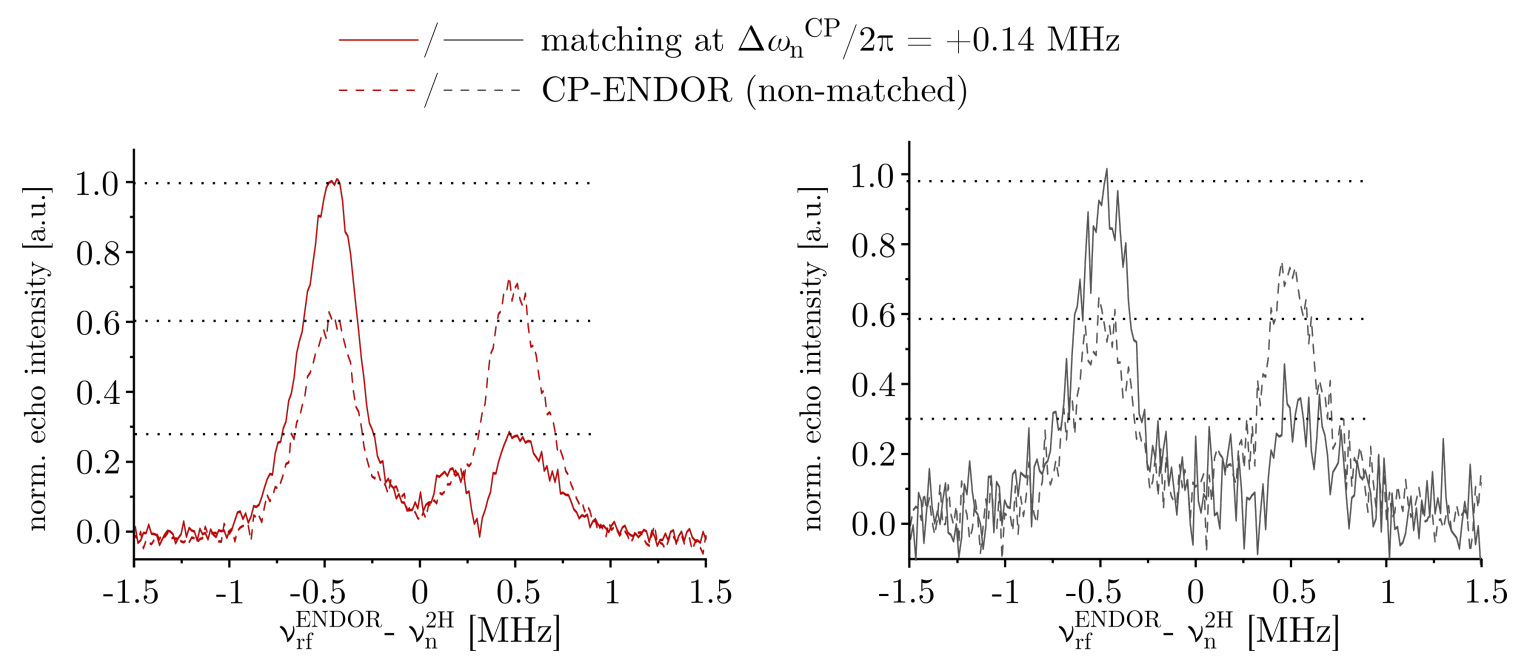

Figure 6.6: Slices from experimental 2D CP-ENDOR spectra. A) 2D WOP CP-ENDOR slices. B) Standard 2D CP-ENDOR slices. Slices for matching at $\Delta \omega_{\mathrm{n}}^{\mathrm{CP}} / 2 \pi=+0.14 \mathrm{MHz}$ (straight lines) and non-matched (dashed lines) are shown. The ratio between matching and no matching $(1: 0.6)$ as well as the asymmetrie factor with matching $(1: 0.3)$ is equal within the uncertainty of the noise for both sequences.

\subsection{Microwave amplitude during spinlock - Numerical predic- tions and experimental trade-off}

At first, matching conditions and ratios between non-matched CP-ENDOR and matched CP-ENDOR were analyzed. For a straight-forward analysis, a single-crystal analogue spin system $(S=1 / 2, I=1)$ under selective, on-resonant excitation of EPR $\beta$ 
$\left(\Delta \omega_{\mathrm{e}} / 2 \pi=0 \mathrm{MHz}\right)$ was considered (Fig. 6.7, A). Fig. 6.7, B and C show quadrants from the simulated 2D CP-ENDOR simulations.

\section{A) Simulated spin system of $S=1 / 2, I=1$ on $E P R \beta$}
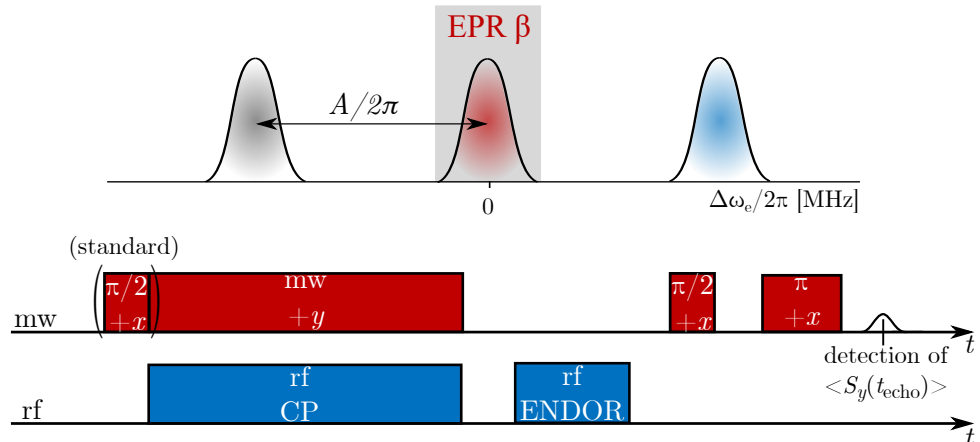

B) $2 D$ WOP CP-ENDOR simulations $\left(\omega_{1 e} / 2 \pi=1.25 \mathrm{MHz}\right)$
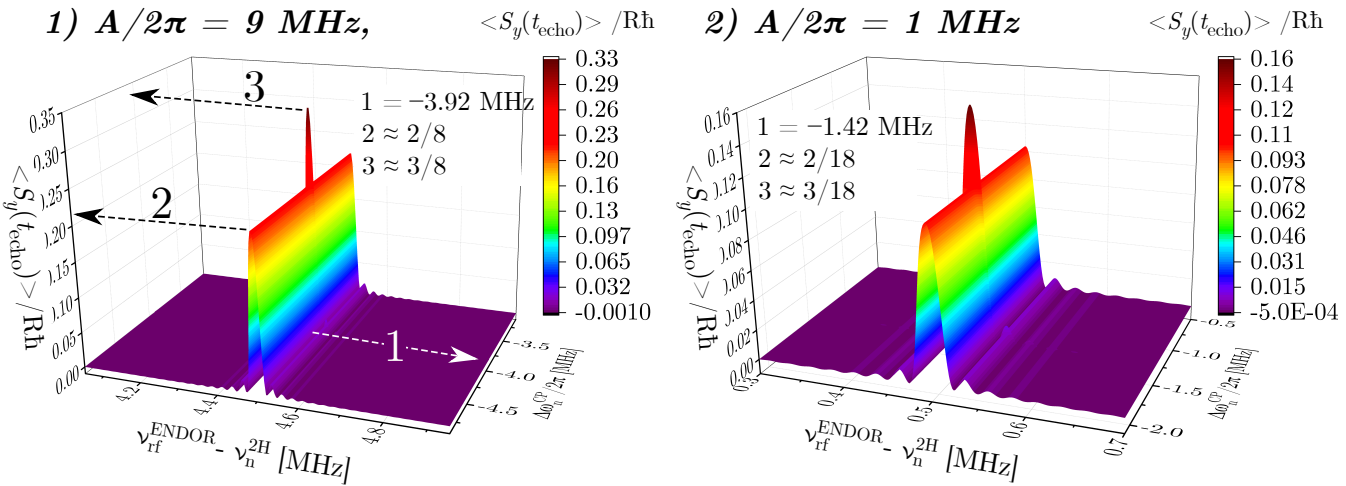

C) $2 D$ standard CP-ENDOR simulations $\left(\omega_{1 e} / 2 \pi=1.25 \mathrm{MHz}\right)$
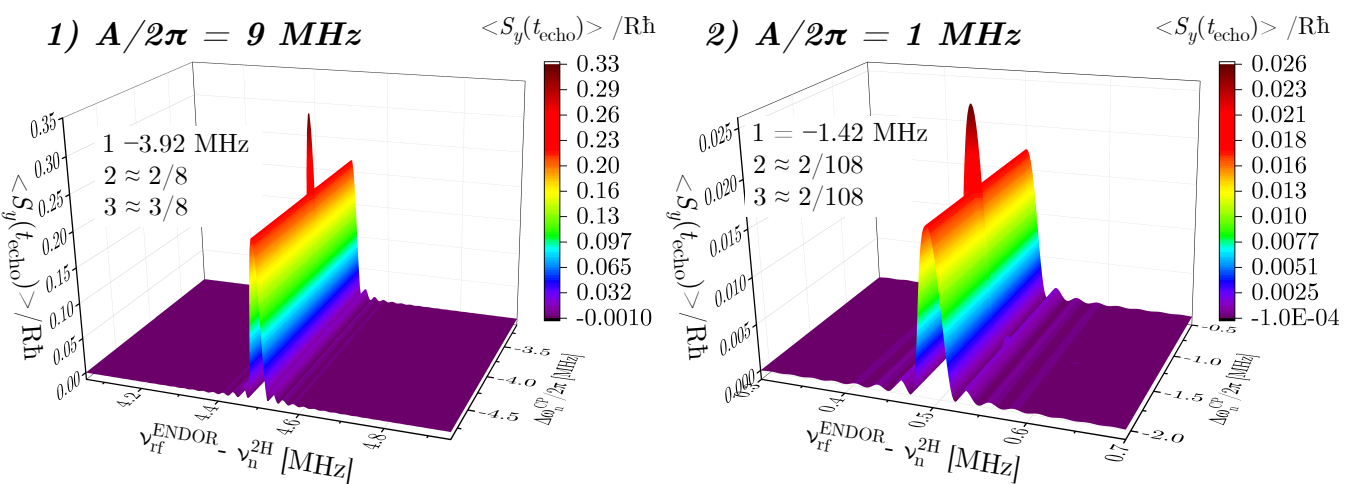

Figure 6.7: Standard CP-ENDOR and WOP CP-ENDOR single crystal simulations for $\boldsymbol{S}=\mathbf{1} / \mathbf{2}, \boldsymbol{I}=\mathbf{1}$. $A$, top). Simulated single crystal spin system with $S=1 / 2$, $I=1, \Delta \omega_{\mathrm{e}} / 2 \pi=0 \mathrm{MHz}(\operatorname{EPR} \beta), \omega_{1 \mathrm{e}} / 2 \pi=1.25 \mathrm{MHz}, \omega_{1 \mathrm{n}} / 2 \pi=13 \mathrm{kHz}, t_{\mathrm{CP}}=200 \mu \mathrm{s}$, $t_{\pi / 2, \mathrm{mw}}=200 \mathrm{~ns}, t_{\pi, \mathrm{rf}}=38 \mu \mathrm{s}$. A, bottom) The simulation maps $\left\langle S_{x}\left(t_{\text {echo }}\right)\right\rangle$ at the respective echo detection time point. $B)$ WOP CP-ENDOR simulations for $A / 2 \pi=9 \mathrm{MHz}(\mathrm{B}, 1)$ and $1 \mathrm{MHz}(\mathrm{B}, 2)$ with $\omega_{1 \mathrm{e}} / 2 \pi=1.25 \mathrm{MHz}$. Extracted values are 1) matching condition (labeled 1 in $\mathrm{B}, 1$ ); 2) intensity of the non-matched CP-ENDOR echo (labeled 2 in B,1); and 3 ) intensity of the matched CP-ENDOR echo (labeled 3 in B,1) $C$ ) Standard CP-ENDOR simulations for $A / 2 \pi=9 \mathrm{MHz}(\mathrm{C}, 1)$ and $1 \mathrm{MHz}(\mathrm{C}, 2)$ with $\omega_{1 \mathrm{e}} / 2 \pi=1.25 \mathrm{MHz}$. 
Each quadrant shows the $\nu_{\mathrm{ENDOR}}-\nu_{\mathrm{n}}^{2 \mathrm{H}}+A / 2 \pi$ ENDOR region $\left(x\right.$-axis) and $\Delta \omega_{\mathrm{n}}^{\mathrm{CP}} / 2 \pi-$ $A / 2 \pi \mathrm{CP}$ offset axis ( $y$-axis) area. The top panel shows WOP CP-ENDOR simulations for the cases of $A / 2 \pi=1 \mathrm{MHz}$ and $9 \mathrm{MHz}(\mathrm{B}, 1$ and $\mathrm{B}, 2)$ with $\omega_{1 \mathrm{e}} / 2 \pi=1.25 \mathrm{MHz}$. The bottom panel shows the same cases for standard CP-ENDOR (C,1 and C,2).

Three values are extracted from the simulations: 1) a matching condition identified at the $\Delta \omega_{\mathrm{n}}^{\mathrm{CP}} / 2 \pi$ value ( $y$-axis) at maximum ENDOR intensity (labeled 1 in $\mathrm{B}, 1$ ); 2 ) intensity of the non-matched ENDOR echo $\left\langle S_{y}\left(t_{\text {echo }}\right)^{\mathrm{SL}}\right\rangle$ (labeled 2 in B,1); and 3) intensity of the matched CP-ENDOR echo $\left\langle S_{y}\left(t_{\text {echo }}\right)^{\mathrm{CP}}\right\rangle$ (labeled 3 in B,1).

Spectra B,1 and C,1, both with $A / 2 \pi=9 \mathrm{MHz}$, show identical non-matched (value 2) and matched CP-ENDOR intensities (value 3). It should be noted, that the observed intensities are consistent with the ones obtained from the numerical simulations in Sec. 5.3.3 of the previous chapter. Further, a matching condition (value 1) is identified at $\Delta \omega_{\mathrm{n}}^{\mathrm{CP}} / 2 \pi=-3.92 \mathrm{MHz}$ for both sequences.

In $\mathrm{B}, 2$ and $\mathrm{C}, 2$ simulations with $A / 2 \pi=1 \mathrm{MHz}$ are shown. The matching condition at $\Delta \omega_{\mathrm{n}}^{\mathrm{CP}} / 2 \pi=-1.42 \mathrm{MHz}$ is identical in both cases. For standard CP-ENDOR, the nonmatched and matched CP-ENDOR echo intensities are $\approx 0.017$ and $\approx 0.026$, respectively. In contrast, WOP CP-ENDOR shows intensities of $\approx 0.10$ and $\approx 0.16$. Thus, for both sequences a ratio between non-matched and matched CP-ENDOR echo intensity of $\approx 1.5$ is observed. The overall intensity of the simulated WOP-ENDOR spectra result $\approx$ six times larger than for standard CP-ENDOR. In summary, we observe sequence independent matching conditions and an improved ENDOR performance predicted for the $A / 2 \pi=1 \mathrm{MHz}$ case, but not for $A / 2 \pi=9 \mathrm{MHz}$. Thus, the WOP CP-ENDOR reveals a dependency on $A / 2 \pi$, which is studied in the following.

For the plot shown in Fig. 6.8, further single-crystal like simulations with varying $A / 2 \pi$ values at constant $\omega_{1 \mathrm{e}} / 2 \pi=1.25 \mathrm{MHz}$ were performed. From each simulation, the non-matched CP-ENDOR intensity (see label 2 in Fig. 6.7, B,1) and CP-ENDOR intensity at the matching condition (see label 3 in Fig. 6.7, B,1) were extracted. The data are summarized in Fig. 6.8, A to obtain the CP-ENDOR echo intensity as a function of $A / 2 \pi$ for standard CP-ENDOR (grey data points) and WOP CP-ENDOR (red data points) under CP matching (open symbols) and non-matched (closed symbols) conditions. The resulting intensity ratio between WOP CP-ENDOR and standard CP-ENDOR is plotted in B. 


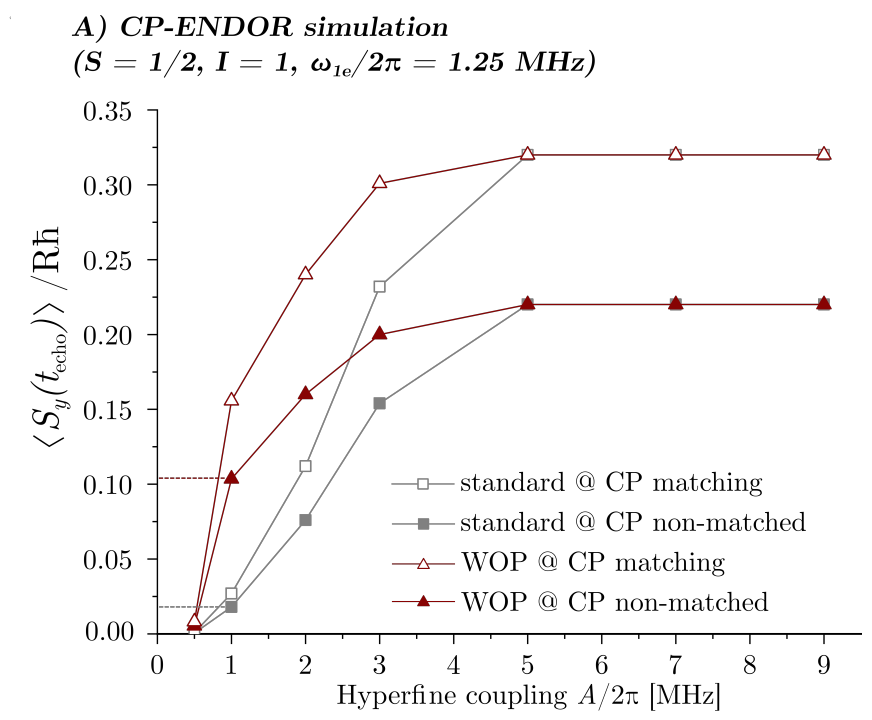

B) Calculated intensity ratio

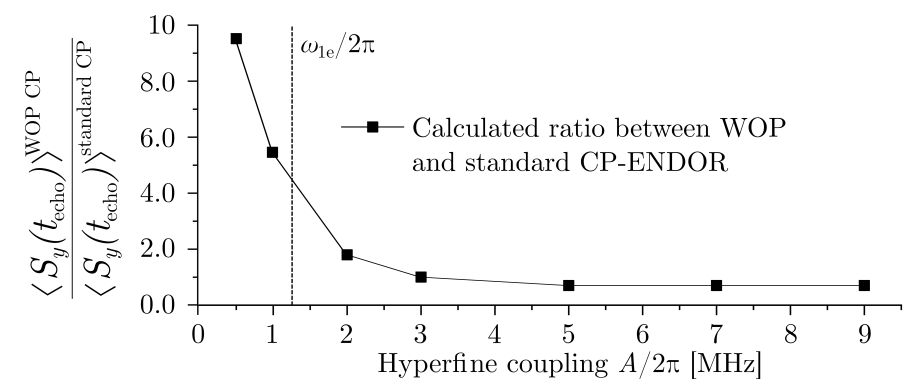

Figure 6.8: Calculated CP-ENDOR signal intensity as a function of $A / 2 \pi$ at constant $\omega_{1 \mathrm{e}} / \mathbf{2} \pi$. Simulation of a single crystal spin system with $S=1 / 2, I=1$, $\Delta \omega_{\mathrm{e}} / 2 \pi=0 \mathrm{MHz}(\mathrm{EPR} \beta), \omega_{1 \mathrm{e}} / 2 \pi=1.25 \mathrm{MHz}, \omega_{1 \mathrm{n}} / 2 \pi=13 \mathrm{kHz}, t_{\mathrm{CP}}=200 \mu \mathrm{s}$, $t_{\pi / 2, \mathrm{mw}}=200 \mathrm{~ns}, t_{\pi, \mathrm{rf}}=38 \mu \mathrm{s}$. The simulation maps $\left\langle S_{x}\left(t_{\text {echo }}\right)\right\rangle$ at the respective echo detection time point which reports (see also Fig. 6.7, A). A) Intensity of standard CP-ENDOR (grey points) and WOP CP-ENDOR (red points) for CP matching (open symbols) and without $\mathrm{CP}$ matching (closed symbols). B) Intensity ratio between WOP CP-ENDOR and standard CP-ENDOR.

Two trends are observed: First, the ratio between CP matched (open symbols) and non-matched (closed symbols) echo intensity remains constant $(\approx 1.5)$ at every $A / 2 \pi$. Second, the intensity difference between WOP CP-ENDOR and standard CP-ENDOR increases with decreasing hfc. For example at $A / 2 \pi=9 \mathrm{MHz}$ their ratio is 1 compared to a ratio of $\approx 10$ at $A / 2 \pi=0.5 \mathrm{MHz}$. The presented enhancement predictions are obtained for $\omega_{1 \mathrm{e}} / 2 \pi=1.25 \mathrm{MHz}$. While the presented enhancement predictions were obtained for a fixed $\omega_{1 \mathrm{e}} / 2 \pi$, the dependency of the ENDOR intensity on $\omega_{1 \mathrm{e}} / 2 \pi$ is demonstrated in the following.

The following considerations focus only on the non-matched CP-ENDOR intensity because a constant factor of $\approx 1.5$ between $\mathrm{CP}$ matched and non-matched signals at every $A / 2 \pi$ was observed previously. Accordingly, the following calculation of non- 
matched CP-ENDOR intensities is assumed to show a similar intensity dependency on $\omega_{1 \mathrm{e}} / 2 \pi$ when performed under CP-maching, however with $\approx 1.5$ times higher absolute signal intensity. In Fig. 6.9, the non-matched ENDOR intensity dependency on $\omega_{1 \mathrm{e}} / 2 \pi$ is analyzed for the WOP CP-ENDOR sequence. Each color refers to a different microwave field strength during the microwave spinlock pulse from $\omega_{1 \mathrm{e}} / 2 \pi=0.5 \mathrm{MHz}$ (yellow) to $5 \mathrm{MHz}$ (red). The general trend shows reduced signal intensity for small hfcs when high $\omega_{1 \mathrm{e}} / 2 \pi$ is applied. In addition, the higher $\omega_{1 \mathrm{e}} / 2 \pi$ the broader the $A / 2 \pi$ range that gets reduced. For example, at $A / 2 \pi=2 \mathrm{MHz}$ the intensity decreases by a factor of $\approx 9$ when increasing $\omega_{1 \mathrm{e}} / 2 \pi$ from 0.5 to $5 \mathrm{MHz}$. Likewise, the full width at half maximum $(\mathrm{FWHM})$ decreases from $\approx 5.5 \mathrm{MHz}$ at $\omega_{1 \mathrm{e}} / 2 \pi=5 \mathrm{MHz}$ to $0.53 \mathrm{MHz} \approx$ at $\omega_{1 \mathrm{e}} / 2 \pi=0.5 \mathrm{MHz}$. In summary, $\omega_{1 \mathrm{e}} / 2 \pi$ scales the affected hfc range/width and the amount of signal reduction.

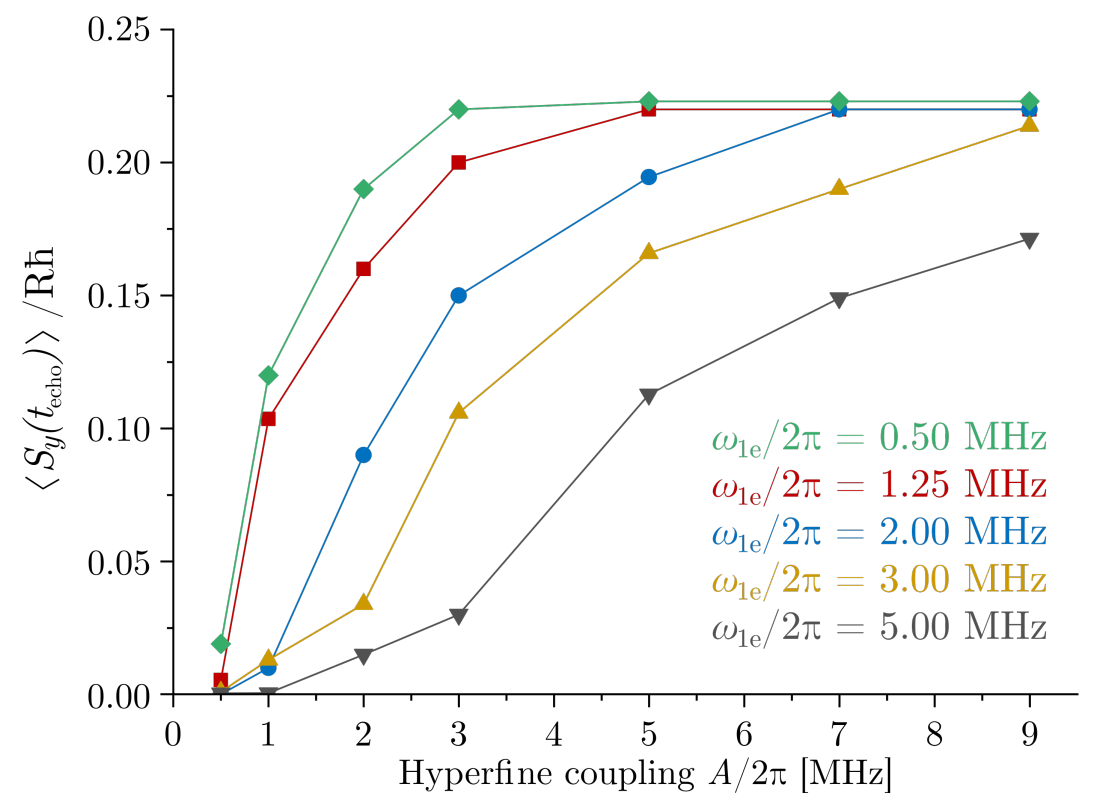

Figure 6.9: Calculated WOP CP-ENDOR intensity (non-matched) as a function of $\omega_{1 \mathrm{e}} / 2 \pi$ and $\boldsymbol{A} / \mathbf{2} \pi$. Simulation of a single crystal spin system with $S=1 / 2$, $I=1, \Delta \omega_{\mathrm{e}} / 2 \pi=0 \mathrm{MHz}(\mathrm{EPR} \beta), \omega_{1 \mathrm{n}} / 2 \pi=13 \mathrm{kHz}, t_{\mathrm{CP}}=200 \mu \mathrm{s}, t_{\pi / 2, \mathrm{mw}}=200 \mathrm{~ns}$, $t_{\pi, \mathrm{rf}}=38 \mu \mathrm{s}$. The simulation maps $\left\langle S_{x}\left(t_{\text {echo }}\right)\right\rangle$ at the respective echo detection time point (see also Fig. 6.7, A). $\omega_{1 \mathrm{e}} / 2 \pi$ is varied between 0.5, 1.25, 2.00, 3.00 and $5.00 \mathrm{MHz}$.

The $\omega_{1 \mathrm{e}} / 2 \pi$ dependency observed in Fig. 6.9 was tested experimentally on ${ }^{2} \mathrm{H}-\mathrm{BDPA}$. For this purpose, 2D WOP CP-ENDOR was performed with a reduced $\omega_{1 \mathrm{e}} / 2 \pi$ of $0.5 \mathrm{MHz}$. The result is shown in Fig. 6.10, A, in comparison to the previously shown spectrum. The $2 \mathrm{D}$ data set of CP-ENDOR with $\omega_{1 \mathrm{e}} / 2 \pi=0.5 \mathrm{MHz}$ is plotted in Fig. 6.10, B. 
A) WOP CP-ENDOR comparison

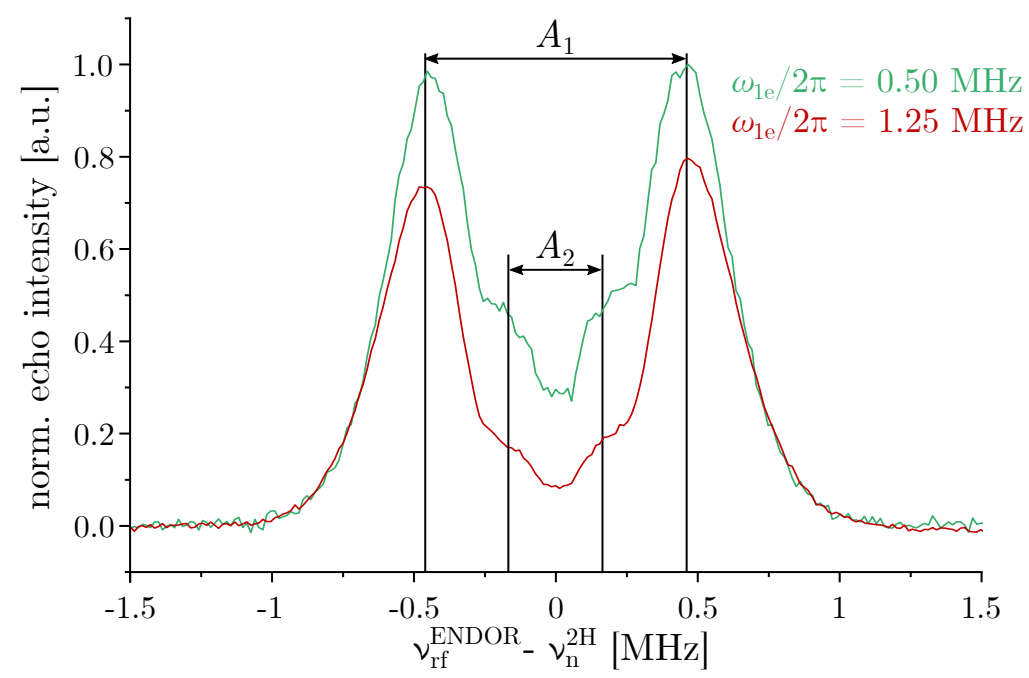

B) Landscape and Contour plot, $\omega_{1 \mathrm{e}} / 2 \pi=0.5 \mathrm{MHz}$

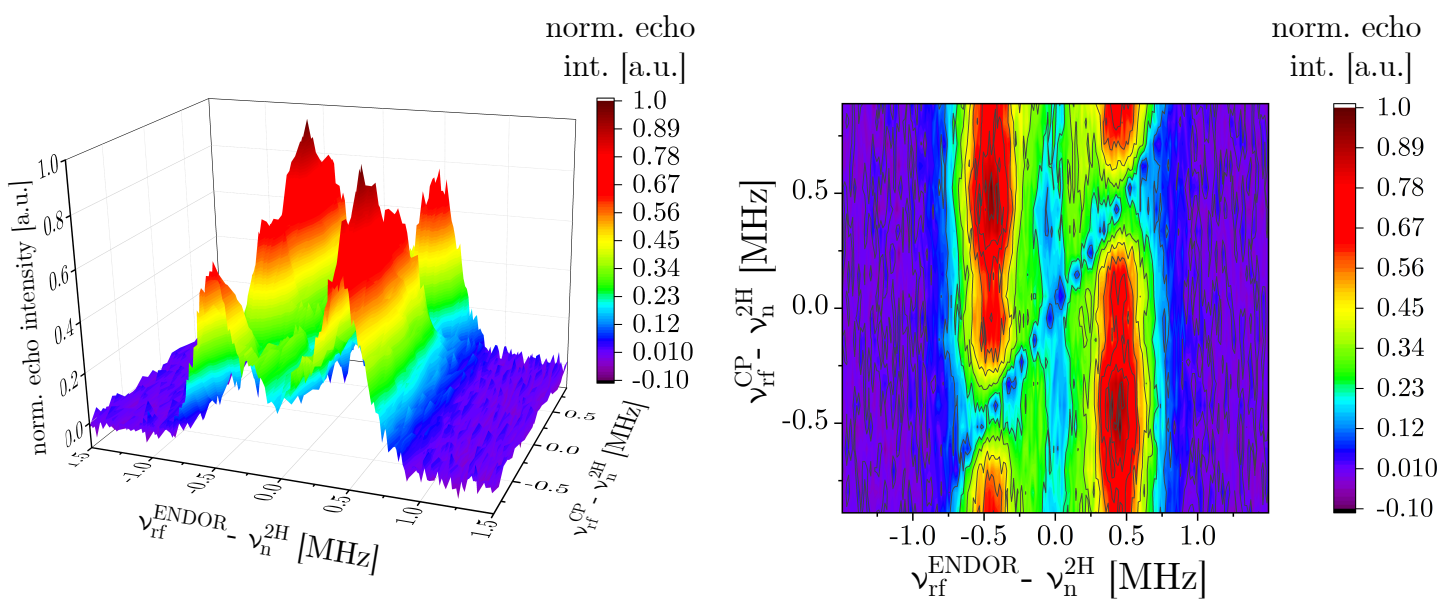

Figure 6.10: WOP CP-ENDOR of ${ }^{2} \mathrm{H}-\mathrm{BDPA}$ with low $\boldsymbol{\omega}_{1 \mathrm{e}} / \mathbf{2} \boldsymbol{\pi}$. A) $0.1 \%$ ${ }^{2} \mathrm{H}-\mathrm{BDPA}$ WOP CP-ENDOR spectrum with $\omega_{1 \mathrm{e}} / 2 \pi=0.5 \mathrm{MHz}$ (green) and $\omega_{1 \mathrm{e}} / 2 \pi=1.25 \mathrm{MHz}$ (red). B) Landscape and contour plots of 2D WOP CP-ENDOR with $\omega_{1 \mathrm{e}} / 2 \pi=0.5 \mathrm{MHz}$. Exp. conditions: $T=80 \mathrm{~K}, \nu_{\mathrm{EPR}}=94 \mathrm{GHz}, t_{\pi / 2, \mathrm{det}}=500 \mathrm{~ns}$, $\omega_{1 \mathrm{e}} / 2 \pi=0.5 \mathrm{MHz}, \omega_{1 \mathrm{n}} / 2 \pi=13 \mathrm{kHz}, t_{\mathrm{CP}}=200 \mu \mathrm{s}, \Delta \omega_{\mathrm{n}}^{\mathrm{CP}} / 2 \pi=-0.9 \mathrm{MHz}$ to $+0.9 \mathrm{MHz}$ (20 steps) $t_{\pi, \mathrm{rf}}=38 \mu \mathrm{s}$, shot repetition time $=25 \mathrm{~ms}, 1 \mathrm{shot} /$ point, random $\mathrm{rf}$ acquisition, 1000 scans (acquisition time $\approx 2.5 \mathrm{~h}$ ).

The summed up ENDOR spectra are scaled to line shape agreement at the edges of the spectrum. A comparison of the relative intensities of $A_{1}: A_{2}$ obtained with $\omega_{1 \mathrm{e}} / 2 \pi=1.25 \mathrm{MHz}$ and $\omega_{1 \mathrm{e}} / 2 \pi=0.5 \mathrm{MHz}$, revealed relative intensities of $4: 1$ and $4: 2$, respectively. This trend is in agreement with the previous predictions (see Fig. 6.9). However, the $\mathrm{S} / \mathrm{N}$ ratio dropped by at least a factor of 2 . The difference is explained by the fact that the excitation bandwidth for WOP CP-ENDOR experiments is proportional to the $\omega_{1 \mathrm{e}} / 2 \pi$ as will be derived in the following section. Accordingly, the 
lower the $\omega_{1 \mathrm{e}} / 2 \pi$, the narrower the excitation bandwidth and the less spins contribute to the detected signal. This mutual dependency results in lower $\mathrm{S} / \mathrm{N}$ for WOP CP-ENDOR experiments performed with reduced $\omega_{1 \mathrm{e}} / 2 \pi$.

\subsection{Central blind spot in CP-ENDOR spectra derived from numerical and analytical excitation profiles}

In this section, the excitation profiles for both the standard and WOP CP-ENDOR sequences are derived from numerical simulations. Additionally, the excitation profile of the WOP CP-ENDOR sequence is analytically obtained. The excitation profile is of particular importance for the later discussion of the central blind spot in the CP-ENDOR spectrum. In general, every applied pulse ( $\mathrm{mw}$ and rf) of a given frequency at a constant magnetic field simultaneously excites a certain frequency range. For inhomogeneously broadened EPR spectra such a mw pulse burns a so-called hole into the EPR spectrum according to the excitation profile of the pulse (introduced in Sec. 2.3 .2 for Davies ENDOR). In Davies ENDOR, the selective $\pi$ preparation pulse inverts spin packets over a frequency bandwidth proportional to the inverse length of the pulse. Following the description in Sec. 2.3.2 and the corresponding illustration in Fig. 2.4, a large ENDOR effect is achieved for hyperfine couplings exceeding the width of the burnt hole and a reduced ENDOR effect is observed for hyperfine couplings within the hole width. In this thesis, a similar suppression mechanism for small hyperfine couplings was identified in CP-ENDOR experiments in which the excitation profile of the preparation step is crucial. However, the excitation bandwidth of the standard and WOP CP-ENDOR sequences cannot be approximated by the inverse length of the pulse, thus the excitation profiles were numerically and analytically determined.

\subsubsection{Numerically derived excitation profiles of CP-ENDOR}

In standard CP-ENDOR, the excitation profile is characterized by the combination of the $\pi / 2$ pulse and subsequent spinlock pulse profile. When omitting the preparation pulse, solely the long mw pulse defines the excitation bandwidth, as is the case for WOP CP-ENDOR. The resulting difference of the excitation profiles is the origin of the observed intensity improvement of WOP CP-ENDOR for small hfc, and will be demonstrated in the following. 
The starting point are numerically derived ENDOR profiles summarized in Fig. 6.11. For this purpose, the introduced $S=1 / 2, I=1$ spin system with $A / 2 \pi=9 \mathrm{MHz}$, $Q / 2 \pi=0 \mathrm{MHz}$ was considered. The excitation profile was obtained from $-\left\langle S_{z}\left(t_{\mathrm{CP}}\right)\right\rangle$ as a function of the electron spin offset $\Delta \omega_{\mathrm{e}}$ after the long mw pulse (see Fig. 6.11, A). In a nutshell, the effect on the initial $z$-magnetization of the long mw pulse with and without a $\pi / 2$ pulse in front was analyzed. Relaxation processes like spectral diffusion were not taken into account, but are known to broaden the excitation profile 98, 99]. Firstly, the profiles of non-matched CP-ENDOR (grey) and non-matched WOP CPENDOR (red) are compared, both with $\omega_{1 \mathrm{e}} / 2 \pi=1.25 \mathrm{MHz}$ (Fig. 6.11, B). For the standard CP-ENDOR sequence, a $\pi / 2$ preparation pulse of 200 ns duration was assumed.

A) Simulation model

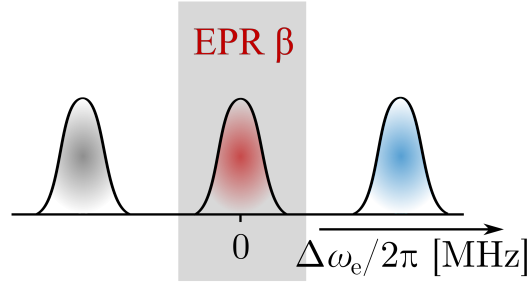

spinlock ENDOR excitation

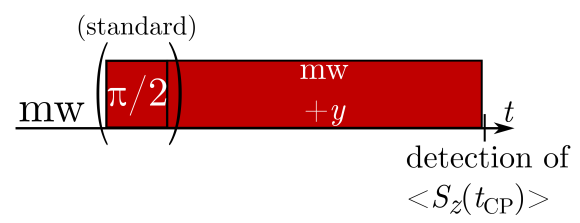

B) Numerically derived excitation profile

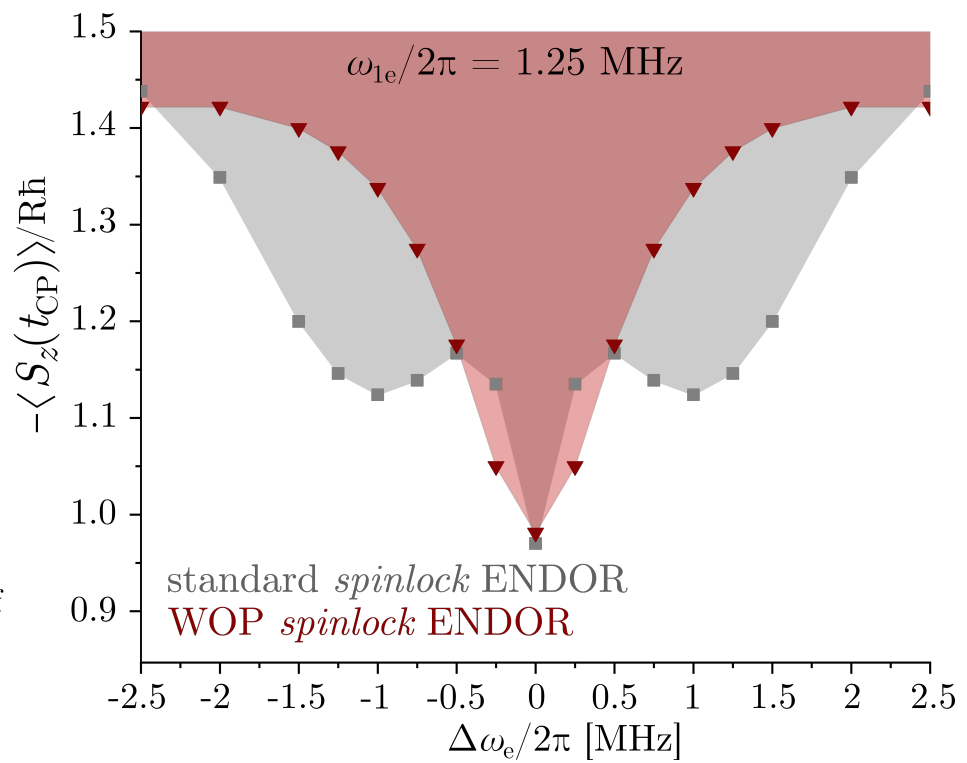

Figure 6.11: Numerically calculated excitation profiles of spinlock ENDOR. A,top) Simulation of a single crystal spin system with $S=1 / 2, I=1$, $\Delta \omega_{\mathrm{e}} / 2 \pi=0 \mathrm{MHz}(\mathrm{EPR} \beta), A / 2 \pi=9 \mathrm{MHz}, Q / 2 \pi=0 \mathrm{MHz}, \omega_{1 \mathrm{e}} / 2 \pi=1.25 \mathrm{kHz}$, $\omega_{1 \mathrm{n}} / 2 \pi=0 \mathrm{kHz}, t_{\mathrm{CP}}=200 \mu \mathrm{s}$. A, bottom) The excitation profiles are obtained from mapping the $z$-magnetization $\left\langle S_{z}\left(t_{\mathrm{CP}}\right)\right\rangle$ as a function of the electron spin offset $\Delta \omega_{\mathrm{e}}$ after the $+\mathrm{y}$ mw pulse. B) The excitation bandwidth of WOP spinlock ENDOR (red) is narrower than the bandwidth of standard spinlock ENDOR (grey) for $\omega_{1 \mathrm{e}} / 2 \pi=1.25 \mathrm{MHz}$ (both) and a $\pi / 2$ preparation pulse of $200 \mathrm{~ns}$ duration (standard CP-ENDOR). Note: The initial density matrix at the beginning of the sequence is defined by $\rho_{0}=-S_{z}$, thus $-\left\langle S_{z}\left(t_{0}\right)\right\rangle$ is obtained to 1.5 .

The simulations were performed without rf amplitude $\left(\omega_{1 \mathrm{n}} / 2 \pi=0 \mathrm{kHz}\right)$ during the long irradiation period. This approach, similar to the non-matched rf offset, results in non CP-enhanced ENDOR spectra. However, even if the rf-offset value is set to satisfy a matching condition, CP transfer does not occur due to lacking rf amplitude. 
In the following, the sequences without rf irradiation during the long mw irradiation period will be referred to as spinlock ENDOR. The colored areas are referred to as excitation profiles created by the excitation period of the sequence. A high ENDOR effect is provided for hfcs surpassing the width of the excitation profile, and, reduced ENDOR effect is achieved for hfcs within the width of the excitation profile, similar to Davies ENDOR (Sec. 2.3.2). Thus, these profiles directly genereate the central blind spot in the spinlock ENDOR spectrum.

At $\Delta \omega_{\mathrm{e}}=0$ the probability $-\left\langle S_{z}\left(t_{\mathrm{CP}}\right)\right\rangle$ amounts to 1 for both sequences. This is rationalized by EPR $\alpha$ and $\gamma$ remaining at their initial spin state $\left(-\left\langle S_{z}\left(I^{\alpha}+I^{\gamma}\right)\left(t_{\mathrm{CP}}\right)\right\rangle=\right.$ $\left.-\left\langle S_{z}\left(I^{\alpha}+I^{\gamma}\right)\left(t_{\text {Start }}\right)\right\rangle=1\right)$ while EPR $\beta$ being selectively saturated $\left(-\left\langle S_{z} I^{\beta}\left(t_{\mathrm{CP}}\right)\right\rangle=0\right)$. The on-resonant component EPR $\beta$ is saturated, the off-resonant components remain unperturbed. For increasing/decreasing electron spin offset values, the monitored $z$ magnetization increases. This is attributed to decreasing off-resonant excitation of $\mathrm{EPR} \beta$. To summarize, the more the excitation frequency is shifted from the resonance position the more the $z$-magnetization remains aligned along the laboratory $z$-axis. However, the amplitude of off-resonant excitation at a certain electron-spin offset values is different for the two sequences. The difference is quantified by a FWHM of $3.34 \mathrm{MHz}$ and $1.25 \mathrm{MHz}$ for standard and WOP spinlock ENDOR, respectively.

Fig. 6.12 shows the results of similar simulations $(S=1 / 2, I=1, A / 2 \pi=9 \mathrm{MHz}$ $\left.Q / 2 \pi=0 \mathrm{MHz}, \omega_{1 \mathrm{e}} / 2 \pi=2.5 \mathrm{MHz}\right)$, yet in this case the rf pulse during spinlock is also considered (Fig. 6.12, A). The simulated profiles for standard CP-ENDOR and WOP CP-ENDOR are summarized in Fig. 6.12, B. The standard CP-ENDOR results (grey) are plotted at positive electron spin offset values and the WOP CP-ENDOR results (red) at negative offset values for a better visual comparison. It should be noted that both profiles individually are symmetrical around $\Delta \omega_{\mathrm{e}}=0$. The CP-matched intensity is plotted in light color and the non-matched intensity in saturated color. 
A) Simulation model

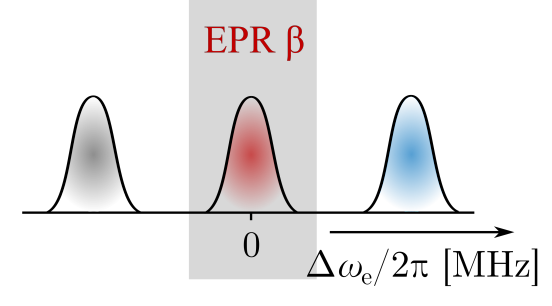

CP ENDOR excitation

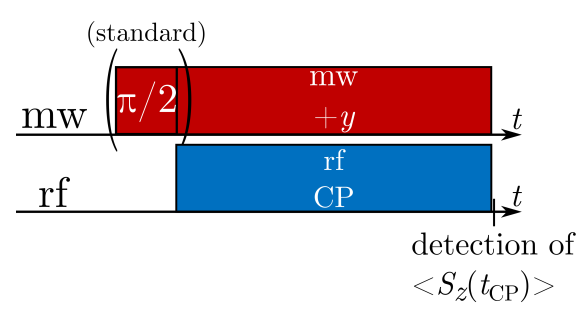

B) Numerically derived excitation profile

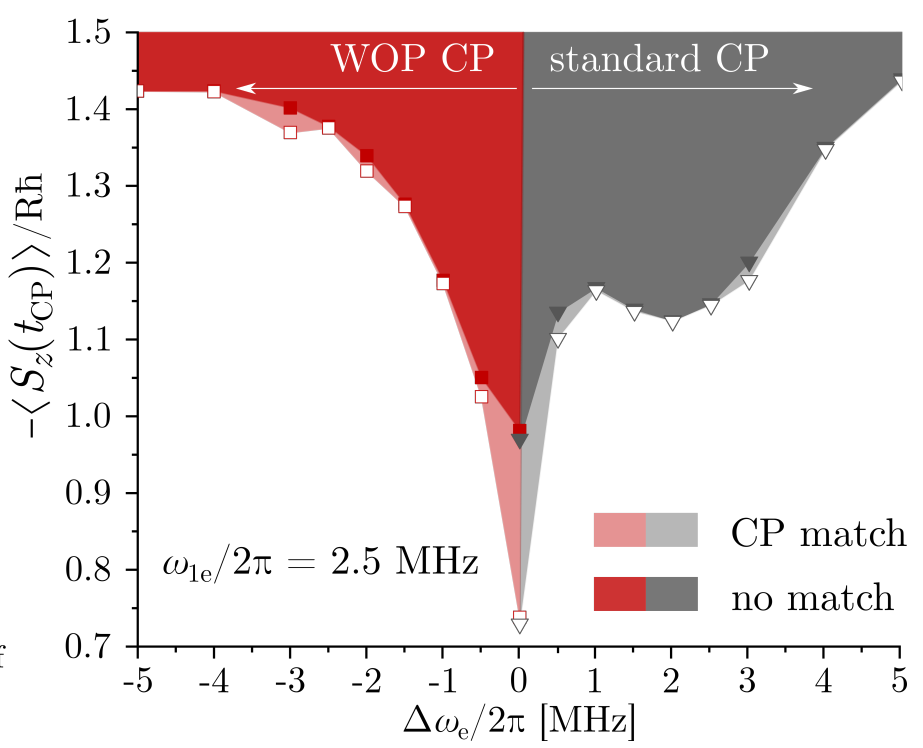

Figure 6.12: Numerically calculated CP-ENDOR excitation profile. A,top) Simulation of a single crystal spin system with $S=1 / 2, \quad I=1$, $\Delta \omega_{\mathrm{e}} / 2 \pi=0 \mathrm{MHz}(\mathrm{EPR} \beta), \omega_{1 \mathrm{e}} / 2 \pi=2.5 \mathrm{MHz}, \omega_{1 \mathrm{n}} / 2 \pi=13 \mathrm{kHz}, A / 2 \pi=9 \mathrm{MHz}$, $Q / 2 \pi=0 \mathrm{MHz}$. A, bottom) A, bottom) The excitation profiles are obtained from mapping the $z$-magnetization $\left\langle S_{z}\left(t_{\mathrm{CP}}\right)\right\rangle$ as a function of the electron spin offset $\Delta \omega_{\mathrm{e}}$ after the $+y \mathrm{mw}$ pulse. B) For a direct comparison half a side of each simulation is shown. Standard CP-ENDOR results (grey) are plotted at positive electron spin offset values and the WOP CP-ENDOR results (red) at negative offset values. The CP-matched intensity is plotted in light color and the non-matched intensity in saturated color. The CP effect mainly results in an increased intensity of the on resonance component. Note: The initial density matrix at the beginning of the sequence is defined by $\rho_{0}=-S_{z}$, thus $-\left\langle S_{z}\left(t_{0}\right)\right\rangle$ is obtained to 1.5.

From the plot we obtain $\left\langle S_{z}\left(t_{\mathrm{CP}}\right)\right\rangle=1$ at $\Delta \omega_{\mathrm{e}}=0$ for both non-matched cases. Thus, in both cases EPR $\beta$ is selectively saturated. The value drops to 0.73 for both $\mathrm{CP}$ matched sequences at $\Delta \omega_{\mathrm{e}}=0$. This difference of 0.27 compared to the non-matched case is due to the $\mathrm{CP}$ effect creating a complex population distribution of the energy levels. Additionally, the major CP effect is identified for the on-resonant component. At $\Delta \omega_{\mathrm{e}} \pm 0.5 \mathrm{MHz}$ only a minor CP effect and at $\Delta \omega_{\mathrm{e}} \pm 1 \mathrm{MHz}$ almost no CP effect is predicted. Accordingly, the burnt hole deepens at the center of irradiation when electron-nuclear cross polarization occurs during the CP step.

Finally, the WOP spinlock ENDOR (no rf) excitation profiles for $\omega_{1 \mathrm{e}} / 2 \pi=1.25 \mathrm{MHz}$ and $2.5 \mathrm{MHz}$ are compared in Fig. 6.13, B (red and blue, respectively). The FWHM directly scales with $\omega_{1 \mathrm{e}} / 2 \pi$ and we obtain a FWHM of $1.25 \mathrm{MHz}$ and $2.5 \mathrm{MHz}$ for $\omega_{1 \mathrm{e}} / 2 \pi=1.25 \mathrm{MHz}$ and $2.5 \mathrm{MHz}$, respectively. 
A) Simulation model

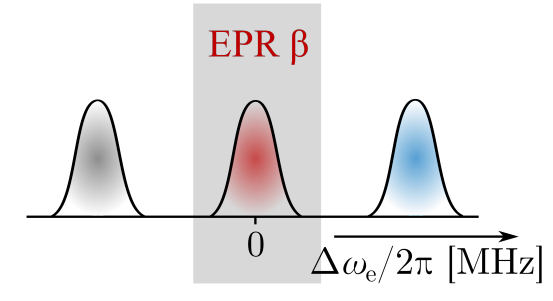

spinlock ENDOR excitation

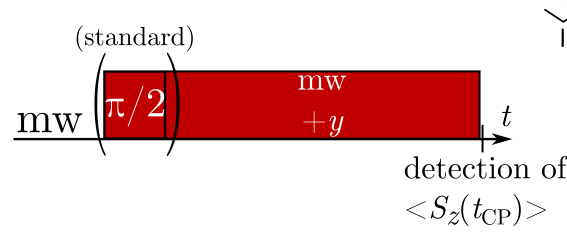

B) WOP spinlock ENDOR excitation profile

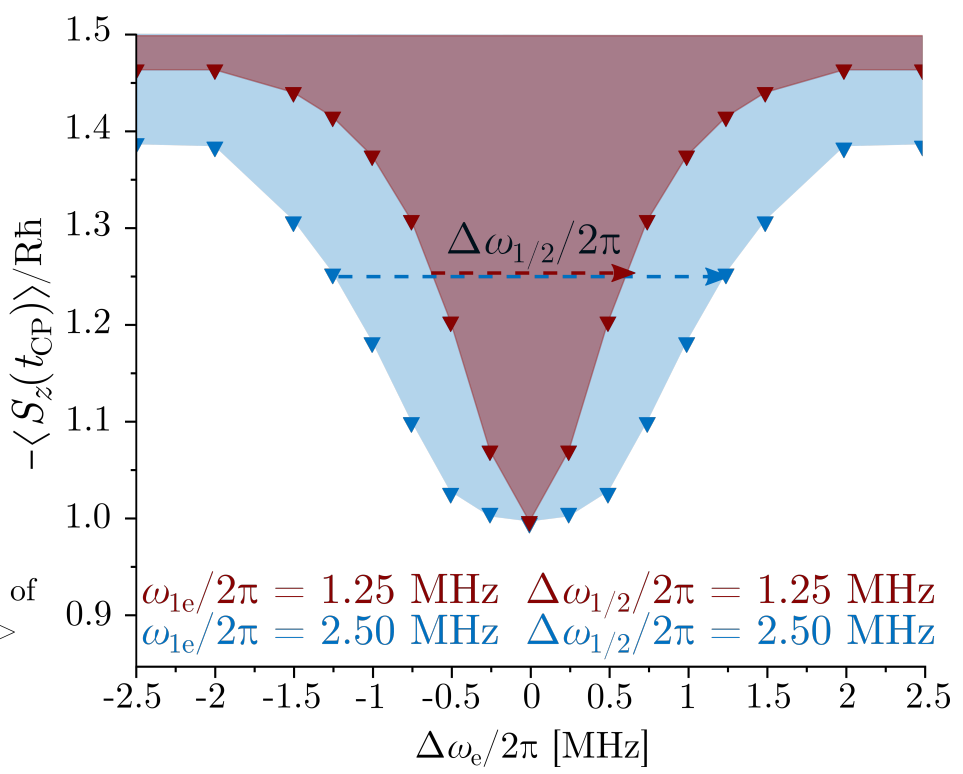

Figure 6.13: Numerically calculated spinlock ENDOR excitation profile dependency on $\omega_{1 \mathrm{e}} / \mathbf{2 \pi}$. A,top) Simulation of a single crystal spin system with $S=1 / 2, I=1, \Delta \omega_{\mathrm{e}} / 2 \pi=0 \mathrm{MHz}(\operatorname{EPR} \beta), \omega_{1 \mathrm{e}} / 2 \pi=1.25 / 2.5 \mathrm{MHz}, \omega_{1 \mathrm{n}} / 2 \pi=0 \mathrm{kHz}$, $A / 2 \pi=9 \mathrm{MHz}, Q / 2 \pi=0 \mathrm{MHz}$. A, bottom) The excitation profiles are obtained from mapping $\left\langle S_{z}\left(t_{\mathrm{CP}}\right)\right\rangle$ as a function of the electron spin offset $\Delta \omega_{\mathrm{e}}$ after the $+y \mathrm{mw}$ pulse. B) The FWHM of WOP spinlock ENDOR scales with $\omega_{1 \mathrm{e}} / 2 \pi$. Thus, FWHMs of 1.25 and $2.5 \mathrm{MHz}$ are obtained for $\omega_{1 \mathrm{e}} / 2 \pi=1.25$ and $2.5 \mathrm{MHz}$, respectively. Note: The initial density matrix at the beginning of the sequence is defined by $\rho_{0}=-S_{z}$, thus $-\left\langle S_{z}\left(t_{0}\right)\right\rangle$ is obtained to 1.5 .

\subsubsection{Analytically derived excitation profile of WOP CP-ENDOR}

In the following section, the excitation profile/central blind spot for the WOP CPENDOR sequence is derived from the analytical treatment. Similarly to the numerical approach, the analytically derived $z$-magnetization after the spinlock pulse is analyzed. The excitation profile for WOP CP-ENDOR is obtained from the magnetization component along the $z$-axis in the laboratory frame at the end of the long mw pulse as a function of the electron spin offset (see green arrows in Fig. 6.14). In recapitulation, the rotation angles for the CP transformation from Sec. 5.2 .2 are:

$$
\begin{array}{lcc}
\text { for } \Delta \omega_{\mathrm{e}}-A \geq 0 & \text { for } \Delta \omega_{\mathrm{e}} \geq 0 & \text { for } \Delta \omega_{\mathrm{e}}+A \geq 0 \\
\quad \theta_{\alpha}=\tan ^{-1}\left(\frac{\omega_{\mathrm{e}}}{\Delta \omega_{\mathrm{e}}-A}\right) & \theta_{\beta}=\tan ^{-1}\left(\frac{\omega_{1 \mathrm{e}}}{\Delta \omega_{\mathrm{e}}}\right) & \theta_{\gamma}=\tan ^{-1}\left(\frac{\omega_{1 \mathrm{e}}}{\Delta \omega_{\mathrm{e}}+A}\right) \\
\text { else } & \text { else } & \text { else } \\
\quad \theta_{\alpha}=\pi+\tan ^{-1}\left(\frac{\omega_{1 \mathrm{e}}}{\Delta \omega_{\mathrm{e}}-A}\right) & \theta_{\beta}=\pi+\tan ^{-1}\left(\frac{\omega_{1 \mathrm{e}}}{\Delta \omega_{\mathrm{e}}}\right) & \theta_{\gamma}=\pi+\tan ^{-1}\left(\frac{\omega_{1 \mathrm{e}}}{\Delta \omega_{\mathrm{e}}+A}\right)
\end{array}
$$


The angles $\theta_{\alpha}, \theta_{\beta}$ and $\theta_{\gamma}$ are interpreted as angles which describe the direction of the effective field to the lab $z$-axis. After this transformation and during the spinlock process, each spin manifold is aligned along the orientation of its effective field. At the end of the CP process, the excitation profile is obtained as the component along the $z$-axis in the lab frame.

Each angle is obtained by an arctangent function of the microwave amplitude $\omega_{1 \mathrm{e}}$, the electron spin offset $\Delta \omega_{\mathrm{e}}$ and the hfc $A$. For the easiest case of EPR $\beta$, the arctangent argument reduces to $\Delta \omega_{\mathrm{e}}$ and $\omega_{1 \mathrm{e}}$. The arctangent correlation is illustrated in Fig. 6.14.

EPR $\beta$ case

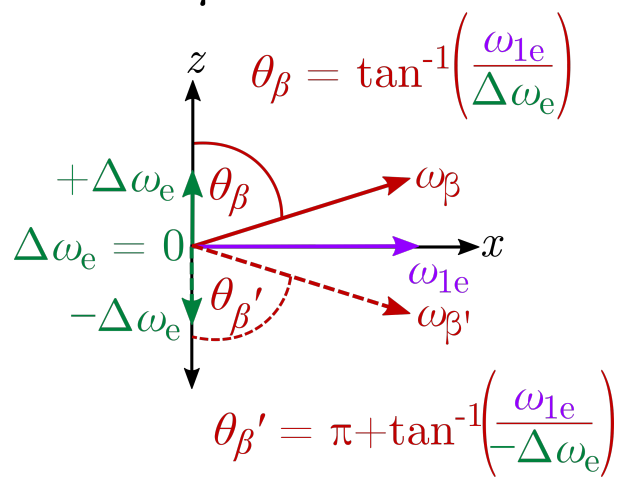

Figure 6.14: Electron spin offset dependency of the effective field illustrated for EPR $\beta$. The effective field $\omega_{\beta}$ is the vector product of the electron spin offset $\Delta \omega_{\mathrm{e}}$ and the microwave field strength $\omega_{1 \mathrm{e}} . \theta_{\beta}$ is defined by the angle closest to the z-axis obtained as the corresponding arctangent function.

Since positive and negative $\Delta \omega_{\mathrm{e}}$ behave similarly in the context of an excitation profile, $\theta_{\beta}$ is defined by the angle closest to the $z$-axis. After a long, selective mw pulse on EPR $\beta$, the density matrix without CP matching is according to Eq. D.20 defined as

$$
\rho^{\prime}\left(t_{\text {spinlock }}\right)=-R I_{z}^{1-4}+R I_{z}^{2-5}+R I_{z}^{3-6}
$$

The transformation back into the doubly rotating frame via the inverse transformation of Eq. 5.23 is given by

$$
\rho\left(t_{\text {spinlock }}\right)=U_{y}^{-1}\left(\theta_{\gamma}, \theta_{\beta}, \theta_{\alpha}\right)\left\{\rho^{\prime}\left(t_{\text {spinlock }}\right)\right\} U_{y}\left(\theta_{\gamma}, \theta_{\beta}, \theta_{\alpha}\right)
$$

and the density matrix in the doubly rotating frame considering only the diagonal elements equals

$$
\rho\left(t_{\text {spinlock }}\right)=-R_{\gamma} I_{z}^{1-4}-R_{\beta} I_{z}^{2-5}-R_{\alpha} I_{z}^{3-6} .
$$


Here, $R_{\alpha}, R_{\beta}$ and $R_{\gamma}$ are proportional to the projection of the magnetization vector onto the effective field for each of the electron spin manifolds according to $R_{\alpha, \beta, \gamma}=R \cos \left(\theta_{\alpha, \beta, \gamma}\right)$ 25. Consequently, Eq. 6.3 is reformulated while considering selective excitation of $\operatorname{EPR} \beta$ and the definitions above of $\theta_{\beta}=\tan ^{-1}\left(\frac{\omega_{1 \mathrm{e}}}{\Delta \omega_{\mathrm{e}}}\right)$ to

$$
\rho_{\mathrm{EPR} \alpha}\left(t_{\text {spinlock }}\right)=-R \cos \left(\tan ^{-1}\left(\frac{\omega_{1 \mathrm{e}}}{\Delta \omega_{\mathrm{e}}}\right)\right) I_{z}^{2-5}
$$

Finally, the $z$-magnetization profile is obtained from

$$
-\left\langle I_{z}^{2-5}\left(t_{\text {spinlock }}\right)\right\rangle=-\left\langle S_{z}\left(t_{\mathrm{CP}}\right)\right\rangle_{\mathrm{EPR} \beta}
$$

Because $\omega_{1 \mathrm{e}} / 2 \pi$ is an experimentally fixed parameter, only the electron spin offset dependency remains. Thus, for a fixed $\omega_{1 \mathrm{e}} / 2 \pi$ the $z$-magnetization dependency on the electron spin offset translates into the excitation profile. This is plotted in Fig. 6.15 for varying $\omega_{1 \mathrm{e}} / 2 \pi$ values of $1.25,2.00,4.00$ and $6.00 \mathrm{MHz}$. The plotting routine can be found in Appendix $\mathrm{G}$ and was written by Prof. Marina Bennati.

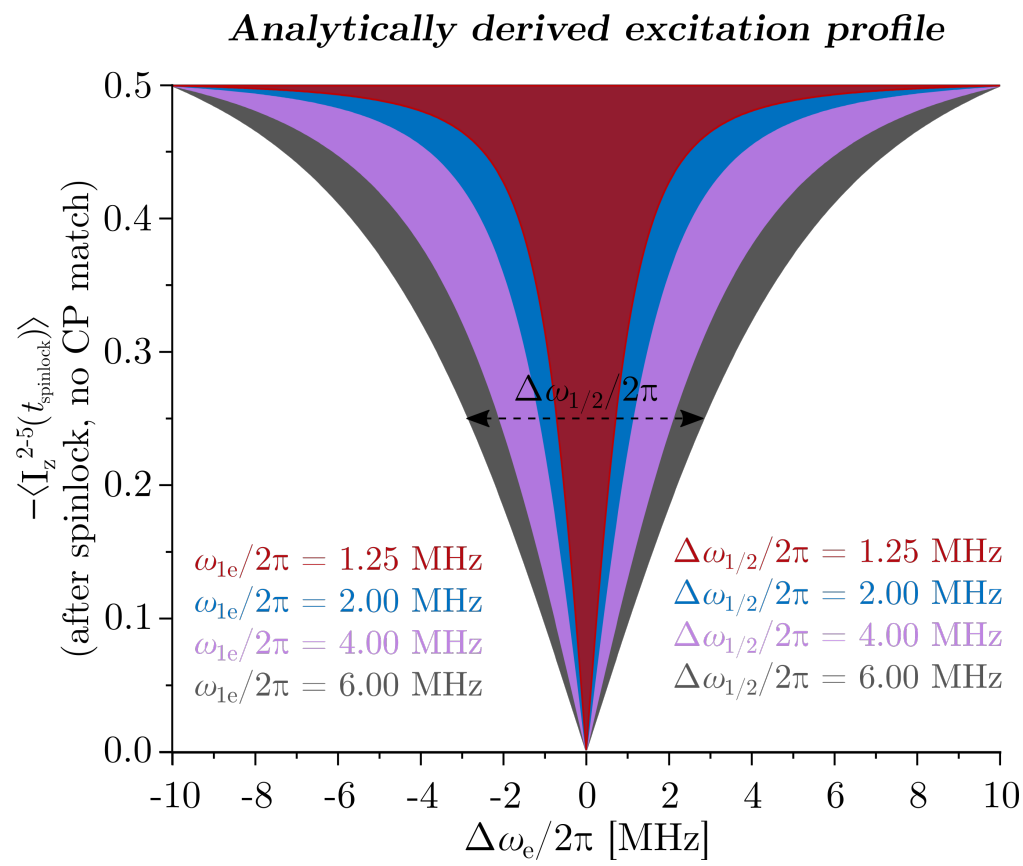

Figure 6.15: Analytically calculated excitation profile of WOP CP-ENDOR. The excitation profiles of WOP CP-ENDOR are calculated for $\omega_{1 \mathrm{e}} / 2 \pi=1.25,2.00$, 4.00 and $6.00 \mathrm{MHz}$. Their FWHM are obtained to $\Delta \omega_{1 / 2} / 2 \pi=1.25,2.00,4.00$ and $6.00 \mathrm{MHz}$, respectively. Note: Here, in contrast to the numerical calculations, only the $z$-magnetization of the EPR $\beta$ transition is mapped. Thus $-\left\langle S_{z}\left(t_{0}\right)\right\rangle_{\mathrm{EPR} \beta}=0.5 \mathrm{R}$.

The FWHM of each profile is equal to the respective $\omega_{1 \mathrm{e}} / 2 \pi$. Thus $\Delta \omega_{1 / 2} / 2 \pi=1.25$, 2.00, 4.00 and $6.00 \mathrm{MHz}$ obtained for $\omega_{1 \mathrm{e}} / 2 \pi$ of $1.25,2.00,4.00$ and $6.00 \mathrm{MHz}$, respec- 
tively. It is noted that only the $z$-magnetization of the $\operatorname{EPR} \beta$ transition was mapped in the analytical calcluation. Therefore, a maximum value of $0.5 \mathrm{R}$ is obtained. Considering selective excitation on $\operatorname{EPR} \beta, \operatorname{EPR} \alpha$ and $\gamma$ are assumed to remain aligned along the lab $z$-axis $\left(-\left\langle S_{z}\left(t_{\mathrm{CP}}\right)\right\rangle_{\mathrm{EPR} \alpha+\gamma}=1 \mathrm{R}\right)$. In contrast, in the numerical calculations the entire $z$-magnetization $\left\langle S_{z}\left(t_{\mathrm{CP}}\right)\right\rangle$ is mapped and maximum values of 1.5 are predicted. Accordingly, the different $y$-offsets in the numerical and analytical treatment is rationalized by either considering the non-excited EPR $\alpha$ and $\gamma$ contributions or not considering them. In summary, the analytically obtained FWHMs are in agreement with the numerical FWHMs.

\subsubsection{Experimentally observed central blind spot in WOP CP-ENDOR}

The excitation profile defines the width of the central blind spot in the CP-ENDOR spectrum, which is conceptually similar to the one obtained in standard Davies ENDOR (for a description see Sec. 2.3.2 and Fig. 2.4). The blind spot effect in the CP-ENDOR spectra and the previously defined dependency of the hole width on $\omega_{1 \mathrm{e}} / 2 \pi$ were examined by spinlock WOP ENDOR experiments summarized in Fig. 6.16.

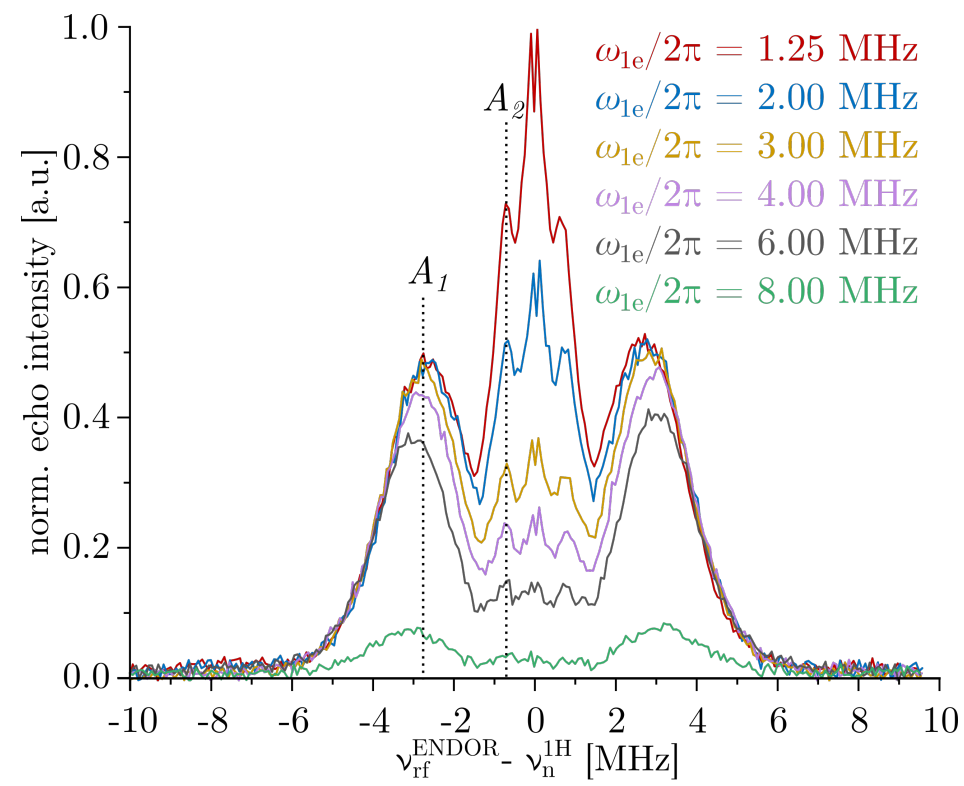

Figure 6.16: Experimental hole function obtained in spinlock WOP ENDOR on ${ }^{1} \mathbf{H}$-BDPA. The hole function is obtained from spinlock WOP ENDOR on $0.1 \%{ }^{1} \mathrm{H}-\mathrm{BDPA}$ for $\omega_{1 \mathrm{e}} / 2 \pi=1.25,2,3,4,6$ and $8 \mathrm{MHz}$. Exp. conditions: $T=80 \mathrm{~K}$, $\nu_{\mathrm{EPR}}=94 \mathrm{GHz}, t_{\pi / 2, \mathrm{det}}=1 /\left(4 \omega_{1 \mathrm{e}} / 2 \pi\right), \omega_{1 \mathrm{n}} / 2 \pi=13 \mathrm{kHz}, t_{\mathrm{CP}}=200 \mu \mathrm{s}, t_{\pi, \mathrm{rf}}=38 \mu \mathrm{s}$, shot repetition time $=25 \mathrm{~ms}, 1$ shot/point, random $\mathrm{rf}$ acquisition, 50 scans each. 
The experiments were performed on protonated BDPA in a polystyrene matrix $(0.1 \%$ $\mathrm{w} / \mathrm{w}$ ), which is the same molecule as presented previously, however all highlighted atoms (green and purple) in Fig. 6.2 are substituted by protons in this case. The molecule was chosen due to its two well-defined hfc tensors from the two nuclei groups $A_{1}$ and $A_{2}$. The hfc size, however, is larger by $\approx 6.5$ compared to the perdeuterated sample, according to the difference in the gyromagnetic ratio between ${ }^{1} \mathrm{H}$ and ${ }^{2} \mathrm{H}$. It allows for ENDOR intensity investigations over a range of $A / 2 \pi \approx 8 \mathrm{MHz}$. Without giving a detailed spectral analysis, the central blind spot is visible from the experiments displayed in Fig. 6.16. The experiment with the lowest $\omega_{1 \mathrm{e}} / 2 \pi$ (red) is normalized to 1 and all experiments up to $\omega_{1 \mathrm{e}} / 2 \pi=6 \mathrm{MHz}$ are scaled to line shape matching at the edges of the spectrum. The $\omega_{1 \mathrm{e}} / 2 \pi=8 \mathrm{MHz}$ (green) spectrum is scaled to the noise level of the $\omega_{1 \mathrm{e}} / 2 \pi=6 \mathrm{MHz}$ spectrum. The highest ENDOR intensities for $A_{1}$ and $A_{2}$ are observed for a low $\omega_{1 \mathrm{e}} / 2 \pi$ (Fig. 6.16, red). The $A_{2}$ intensity drops continuously with increasing $\omega_{1 \mathrm{e}} / 2 \pi$ until it reaches approx. $10 \%$ of the initial intensity. Above $\omega_{1 \mathrm{e}} / 2 \pi=3 \mathrm{MHz}$ the maximum peak intensity of the $A_{1}$ tensor not only gets diminished, but the maximum peak position is also shifted due to partial suppression. Finally, at $\omega_{1 \mathrm{e}} / 2 \pi=8 \mathrm{MHz}$ the central hole width is similar to the hfc range and all ENDOR lines are suppressed by the hole. Thus, the ENDOR spectrum shows very low $\mathrm{S} / \mathrm{N}$. This trend is in agreement with the predictions from analytical and numerical predictions.

At this point, the relation between excitation bandwidth and $\mathrm{S} / \mathrm{N}$ ratio of the ENDOR spectrum must be emphasized. Larger excitation bandwidths result in more spins contributing and in turn a larger signal is obtained. Nevertheless, it becomes evident, that although the excitation bandwidth increases from $\omega_{1 \mathrm{e}} / 2 \pi=1.25 \mathrm{MHz}$ to $8 \mathrm{MHz}$, the $\mathrm{S} / \mathrm{N}$ strongly decreases. Here, the central blind spot crucially reduces the ENDOR efficiency and thus the benefit of exciting more spins gets compromised.

\subsection{Discussion of WOP CP-ENDOR investigations}

The comparative standard and WOP CP-ENDOR experiments on the perdeuterated BDPA powder sample, together with the investigated simulations, demonstrate that WOP CP-ENDOR is superior to standard CP-ENDOR in detecting medium/small hfcs (Fig. 6.4). The improvement is derived from different excitation profiles creating a hole of different widths in the center of the spectrum (Fig. 6.11). An indication that there is a central blind spot in standard CP-ENDOR, and the fact that increased $\omega_{1 \mathrm{e}} / 2 \pi$ reduces the intensity of small hfcs was stated by RizzATO et al.25. 
Indeed, similar to the $\pi$ preparation pulse in Davies ENDOR, a so-called hole is also burnt into the inhomogeously broadened EPR line in CP-ENDOR as illustrated in Fig. 6.17, A. The origin of the central blind spot in Davies ENDOR spectra is illustrated in Fig. 2.4 and discussed in the corresponding Sec. 2.3.2 in Chapter 2. The bandwidth of this hole directly generates the central blind spot in the ENDOR spectrum, causing and the observed ENDOR intensity to get reduced for $|A|,<$ hole width (Fig. 6.17, B). This suppression mechanism is identical to CP-ENDOR experiments.

\section{A) Excitation profile}

\section{B) Central blind spot in ENDOR}

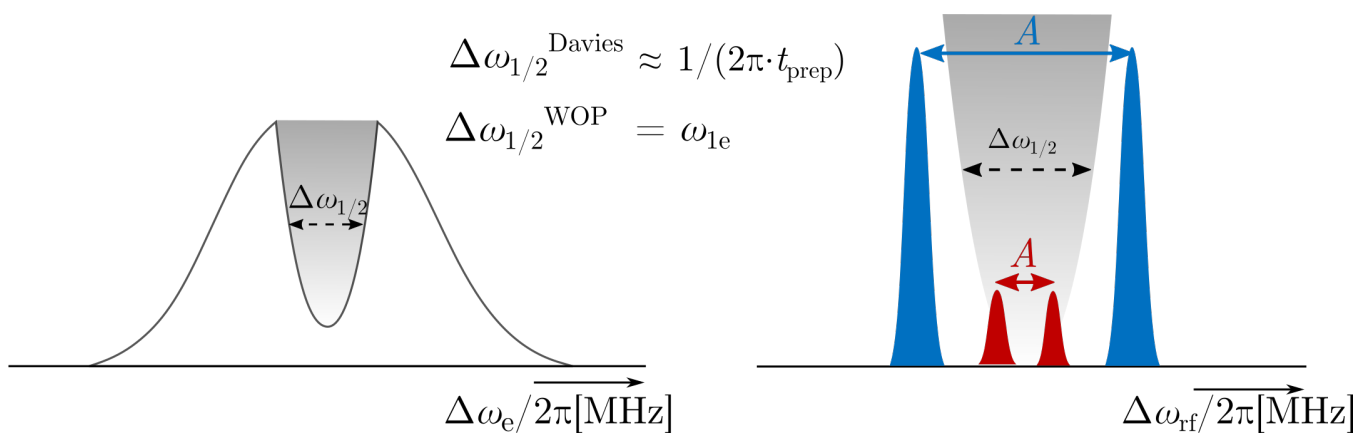

Figure 6.17: Illustration of hole burning and central blind spot in Davies ENDOR and WOP CP-ENDOR spectroscopy. A) In Davies ENDOR and CPENDOR, the preparation pulse burns a hole in the EPR spectrum. The width of the holes are proportional to the inverse of the pulse length and the microwave field strength $\omega_{1 \mathrm{e}} / 2 \pi$ for Davies and WOP CP-ENDOR, respectively. $B$ ) The bandwidth of the burnt hole directly determines the width of the central blind spot in the ENDOR spectrum.

From the simulations, a narrower excitation profile of WOP CP-ENDOR than for standard CP-ENDOR was determined (Fig. 6.11), and, reproduce the obtained enhancement factor of five for ${ }^{2} \mathrm{H}-\mathrm{BDPA}$ (Fig. 6.4). From the numerical excitation profile studies it is concluded that cross polarization occurs predominantly for the on-resonant component, resulting in a deeper hole after the CP transfer (Fig. 6.12). It must be emphasized, that the central blind spot is intrinsically included in the simulation because the excitation bandwidth and thus off-resonant components depend on $\omega_{1 \mathrm{e}} / 2 \pi$.

In summary, the preparation $\pi / 2$ pulse prior to the spinlock pulse significantly broadens the excitation profile of standard CP-ENDOR. In principle, this can be overcome by decreasing the length of this $\pi / 2$-pulse pulse, though this requires higher $\omega_{1 \mathrm{e}} / 2 \pi$. Higher $\omega_{1 \mathrm{e}} / 2 \pi$, in turn, reduces the selectivity of the pulse, which is mandatory for the following CP transfer to occur (see also Chapter 5). Consequently, this becomes unfeasible for the application to small hfcs. Therefore, WOP CP-ENDOR provides a valuable solution to this issue. 
Since the $\omega_{1 \mathrm{e}} / 2 \pi$ in WOP CP-ENDOR experiments directly scales the width of the central blind spot, this parameter should be chosen according to the expected hfc-range in order to minimize the blind spot. The larger the $\omega_{1 \mathrm{e}} / 2 \pi$, the broader the blind spot and the broader the range of reduced hfc in the ENDOR spectrum (Fig. 6.13 and Fig. 6.16). However, although theoretically possible, one can not arbitrarily minimize $\omega_{1 \mathrm{e}} / 2 \pi$. Lower $\omega_{1 \mathrm{e}} / 2 \pi$ leads to a narrower excitation bandwidth, meaning a reduced number of spins participating in the ENDOR sequence. This will in turn also result in lower $\mathrm{S} / \mathrm{N}$ (Fig. 6.10). Accordingly, $\omega_{1 \mathrm{e}} / 2 \pi$ has to be optimized with respect to the target hfc range in order to find a compromise between number of spins contributing to the signal and width of the central blind spot.

A very narrow excitation bandwidth, though, is desired for ENDOR studies of orientationselection. Alongside the narrower excitation bandwidth of the preparation step, the excitation bandwidth during detection is also reduced when reducing $\omega_{1 \mathrm{e}} / 2 \pi$. The general excitation bandwidth of the $\pi / 2$ and $\pi$ pulse of the Hahn-echo detection step are once again proportional to the inverse of the pulse length. Since all pulses have the same constant amplitude in the conventional WOP CP-ENDOR setup, their pulse length is defined by $t_{\pi / 2, \mathrm{mw}}=1 /\left(4\left(\omega_{1 \mathrm{e}} / 2 \pi\right)\right)$ and $\left.t_{\pi, \mathrm{mw}}=1 /\left(2\left(\omega_{1 \mathrm{e}} / 2 \pi\right)\right)\right)$. Accordingly, the lower $\omega_{1 \mathrm{e}} / 2 \pi$, the narrower the excitation bandwidth of the detection pulses. Moreover, longer pulses in the detection step implicate longer $\tau$ evolution time between the two pulses to separate the Hahn-echo from the hardware dead time and free induction decay (for very long pulses, there can be an FID). This extends the duration in which the spin system relaxes (see also Subsec. 4.2.4.1). For ${ }^{2} \mathrm{H}$ BDPA at $T=80 \mathrm{~K}$ relaxation times of $T_{2 \mathrm{e}} \approx 1.6 \mu \mathrm{s}$ and $T_{1 \mathrm{e}}=8 \mathrm{~ms}$ were determined (data not shown) and the Hahn-echo detection pulses for CP-ENDOR experiments were separated by $\tau=1.1 \mu \mathrm{s}$. Accordingly, only $\approx 50 \%$ of the magnetization is detected. Both narrower excitation bandwidth and longer relaxation period compromise the overall $\mathrm{S} / \mathrm{N}$. It is worth to note again that we did not consider spectral diffusion, which is known to reduce the burnt hole 29$]$.

A fundamentally similar central blind spot and $\omega_{1 \mathrm{e}} / 2 \pi$ influence on the blind spot width is observed in electron-electron double resonance detected NMR (ELDOR-detected NMR), firstly published by SCHOSSELER et al. .100$]$. ELDOR-detected NMR is a method which probes nuclear transitions on the basis of driving forbidden electron spin transitions via a high-turning-angle (hta) pulse on a second microwave frequency. Thus, in this technique also a long mw irradiation pulse is applied. The authors report that besides the intended forbidden transitions, allowed transitions are also driven by 
this hta pulse which gives rise to a central blind spot around the irradiation frequency. Later, Cox et al.101] stated that the central blind spot does not depend on the length of the pulse, but on the mw field strength and can be approximated by Lorentzian function. This statement is also true for WOP CP-ENDOR. The major difference between the hole in ELDOR-detected NMR and WOP CP-ENDOR is its consequence for the spectral line shape. In ELDOR-detected NMR, the hole burning results in an additional matrix peak, which then defines the lower limit of the detectable coupling size. The direct observation of this matrix peak allows fitting of the central hole function as a Lorentzian and the linear correlation between FWHM and $\omega_{1 \mathrm{e}} / 2 \pi$ is used to measure the applied field strength. In CP-ENDOR spectroscopy, the central blind spot creates a reduced intensity in the center of the spectrum. Thus, it cannot be obtained and fitted in a straight-forward manner, but is derived based on a semi-theoretical approach (Figs. 6.13, 6.15).

Based on single-crystal simulations (Fig. 6.7), it was verified that even without the first $\pi / 2$ preparation pulse, the CP mechanism is conserved, because matching conditions and CP asymmetry are observed as in standard CP-ENDOR. Consequently, the model of the electron spin in the dressed state interacting with the nuclear spin in its bare state still holds. This assumption has been tested numerically, by comparing the simulated CP-ENDOR spectra calculated by diagonalization of the Hamiltonian in the electron-nuclear tilted frame, with the results from diagonalizing the Hamiltonian in the electron spin tilted frame only. No significant difference was observed, thus validating our model qualitatively (data not shown).

A more general quantum mechanical description of an electron-nuclear spin system under (long) microwave irradiation has been published by JESCHKE ${ }^{102}$. The author describes the evolution of an $S=1 / 2, I=1 / 2$ electron-nuclear two spin system with arbitrary hfcs during mw excitation. JESCHKE not only derives the evolution for an arbitrary initial state, but also specifically for Boltzmann equilibrium $\left(\rho=-S_{z}\right)$ which is analogous to our WOP CP-ENDOR case. In contrast to the treatment in this thesis, the general Hamiltonian in this publication includes the secular part of the hfc ( $B_{x}$ and $B_{y}$ in Eq. 2.27). Thus, in the first step the general Hamiltonian is transformed such that the secular terms reduce to a parameter $B^{\prime}$ depending on $B_{x}$ and $B_{y}$, necessary for the well-known nuclear orientation via electron spin locking (NOVEL) experiment 103, 104]. In the next step of the treatment, the Hamiltonian is transferred into a nuclear tilted frame where the nuclear spin part of this general Hamiltonian is 
diagonalized. Subsequently, this Hamiltonian is the basis to find the electron spin tilted frame Hamiltonian under microwave irradiation. The important result for the context of this thesis is that a selective mw pulse along $x$ generates (among others) $x$ and $y$ electron spin coherence scaling with combinations of sine and cosine functions for $x$ and $y$, respectively (Section 2.4 and Eq. 29 in JESCHKE ${ }^{[02}$ ). However, the rf irradiation simultaneous to the long mw pulse was not considered in this treatment ${ }^{[102]}$. Lastly, according to the comparison of all ENDOR sequences in Fig. 6.2, a benefit of WOP CP-ENDOR in comparison with Mims ENDOR was not obviously observed, as the $\mathrm{S} / \mathrm{N}$ ratio and line shape are identical for the radical under investigation. However, the example shows a Mims ENDOR spectrum with a $\tau$ value of $200 \mathrm{~ns}$, which is just sufficient to ensure its outer Mims blind spots do not interfere with the hfe tensors. Often such a condition cannot be found causing the Mins ENDOR spectrum to become distorted. The radical in focus of the next chapter represents one of the cases where the Mims ENDOR spectrum gets distorted no matter which $\tau$ value is set in the Mims ENDOR experiment. Here, advantages of the WOP CP-ENDOR will become more evident. 


\section{${ }^{2} \mathrm{H}$ WOP CP-ENDOR of $\mathrm{ND}_{2} \mathrm{Y}_{731}$ from $E$. coli RNR}

In the following chapter the WOP CP-ENDOR performance on a biological system is demonstrated. Firstly, comparative ENDOR experiments are discussed in terms of signalto-noise ratio and line shape. Secondly, orientation-selective WOP CP-ENDOR spectra of $\mathrm{ND}_{2} \mathrm{Y}_{731}$ in $E$. coli ribonucleotide reductase are presented for the investigation of the $\mathrm{ND}_{2}$-conformation. The determination of the $\mathrm{ND}_{2}$-conformation in this thesis was used

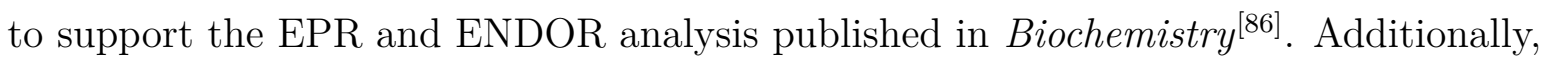
the CP simulation routine is extended to orientation-selective CP-ENDOR spectra.

\subsection{WOP CP-ENDOR investigations of $\mathrm{ND}_{2} \mathrm{Y}_{731}{ }^{\bullet}$}

Site-specifically incorporated $\mathrm{ND}_{2} \mathrm{Ys}$ in E. coli RNR provide a useful tool to spin trap the electron on PCET pathway residues 79,85 . The pathway radical $\mathrm{ND}_{2} \mathrm{Y}_{731}$ • has previously been characterized by EPR, ENDOR and density functional theory (DFT) 17, 18. Based on these studies, mechanistic information on the PCET pathway, such as the hydrogen bond environment at the individual pathway radicals, have been established 16 18. Nonetheless, experimental evidence of the DFT-predicted planar configuration of the amino group remained elusive. In principle, orientation-selective ENDOR spectroscopy is well suited for answering the question of the conformation of the $\mathrm{NH}_{2} \mathrm{Y}$ radical intermediate. Indeed, the experimental approach relies on $\mathrm{H}_{2} \mathrm{O} \rightarrow \mathrm{D}_{2} \mathrm{O}$ buffer exchange and detection of the deuterium ENDOR resonances 16,17 . This results in small hfcs of the $\mathrm{ND}_{2}$ deuterons, thus, Mims ENDOR becomes the method of choice. However, the spectroscopic limitations of conventional ENDOR spectroscopy, above all the line shape distortions due to blind spots in Mims ENDOR, mask a major part of the hf-tensor contributions of the amino group. Since the CP-ENDOR experiment is 
not affected by such blind spots it is better suited to investigate the $\mathrm{ND}_{2}$ structure from orientation-selective CP-ENDOR spectra. The WOP CP-ENDOR sequence should aim at overcoming previous spectroscopic limitations and facilitate structural interpretations from $94 \mathrm{GHz}$ WOP CP-ENDOR on the long-standing $\mathrm{ND}_{2} \mathrm{Y}$ mutation at $\mathrm{Y}_{731}-\alpha 2$. In parallel, a new labeling strategy using $\mathrm{D}_{6}-\mathrm{NH}_{2} \mathrm{Y}_{731}-\alpha 2$ in $\mathrm{H}_{2} \mathrm{O}$ buffer allowed for ${ }^{1} \mathrm{H}$ Davies ENDOR spectroscopy at $34 \mathrm{GHz} / 1.2 \mathrm{~T}$ and multifrequency EPR studies performed in our group 86 . However, the incorporation of $\mathrm{D}_{6}-\mathrm{NH}_{2} \mathrm{Y}_{731}$ in $\alpha 2$ results in low radical yields, thus, orientation-selective ENDOR spectroscopy at 94/1.2 T GHz became compromised by sensitivity issues. Since $34 \mathrm{GHz}$ ENDOR spectra are insensitive to precise tensor-orientation constraints and the determination of hf tensor parameters and particularly tensor orientations from solely fitting field-sweep ESE spectra is an even more challenging task 4 , the experimental results from the ${ }^{1} \mathrm{H}$ and ${ }^{2} \mathrm{H}$ studies were concomitantly analyzed.

In this thesis, the ${ }^{2} \mathrm{H}$ WOP CP-ENDOR investigations on $\mathrm{ND}_{2} \mathrm{Y}_{731}$ ' demonstrate the application of a novel pulse-sequence on a biological system. Thus, they were not included in our recent publication $\overline{86}$.

\subsubsection{WOP CP-ENDOR performance}

Fig. 7.1 shows a comparison of ENDOR sequences under identical acquisition conditions. The radical was obtained from hand freeze-quenching as described in Section 4.1.3 and the radical yield is estimated to be $33 \%$ of the total spin (see also Section 4.1.3). Two Mims (grey and blue), one Davies (green) and one WOP CP-ENDOR spectrum (red) were recorded at $T=10 \mathrm{~K}$ with the same shot repetition time of $100 \mathrm{~ms}$, random ENDOR rf acquisition and 1800 scans each. WOP CP-ENDOR was run in 2D from a PulseSPEL routine. The setup was chosen in analogy to CP-ENDOR experiments of ${ }^{2} \mathrm{H}$-BDPA. A mw amplitude of $\omega_{1 \mathrm{e}} / 2 \pi=1.25 \mathrm{MHz}$ and $t_{\mathrm{CP}}=200 \mu$ s spinlock pulse duration were used for the CP step. The read-out was achieved via Hahn-echo detection with $t_{\pi / 2 \text {,det }}=200 \mathrm{~ns}-\tau-t_{\pi, \operatorname{det}}=400 \mathrm{~ns}-\tau-$ echo $\left(\omega_{1 \mathrm{e}} / 2 \pi=1.25 \mathrm{MHz}\right)$. For $2 \mathrm{D}$ data acquisition, the rf offset during spinlock was incremented in 36 steps between $\Delta \omega_{\mathrm{n}}^{\mathrm{CP}} / 2 \pi=-2.3 \mathrm{MHz}$ to $+2.3 \mathrm{MHz}$. The chosen $\Delta \omega_{\mathrm{n}}^{\mathrm{CP}} / 2 \pi$ range was determined from an eNCP experiment shown in Appendix $\mathrm{H}$. The rf offset was incremented linearly with 50 scans per $\Delta \omega_{\mathrm{n}}^{\mathrm{CP}} / 2 \pi$. The obtained experimental 2D WOP CP-ENDOR spectrum (see Fig. 7.7, left column) were summarized to obtain only the ENDOR dimension. The setup provides a compromise between width of the central blind spot and signal-to-noise 
ratio: A weaker mw amplitude during spinlock implies a narrower central blind spot and thus a higher intensity for small hfcs. It also implies a smaller excitation bandwidth and lower $\mathrm{S} / \mathrm{N}$ according to the results of the previous Chapter (see Fig. 6.10).

A) Radical structure<smiles></smiles>

B) ESE-detected field sweep spectrum

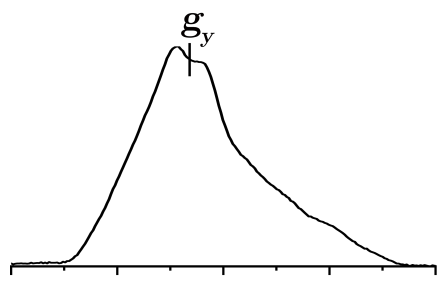

334253345033475 magnetic field $[\mathrm{G}]$

\section{C) ${ }^{2} \mathrm{H}$-ENDOR at $B_{0} / / g_{y}$}

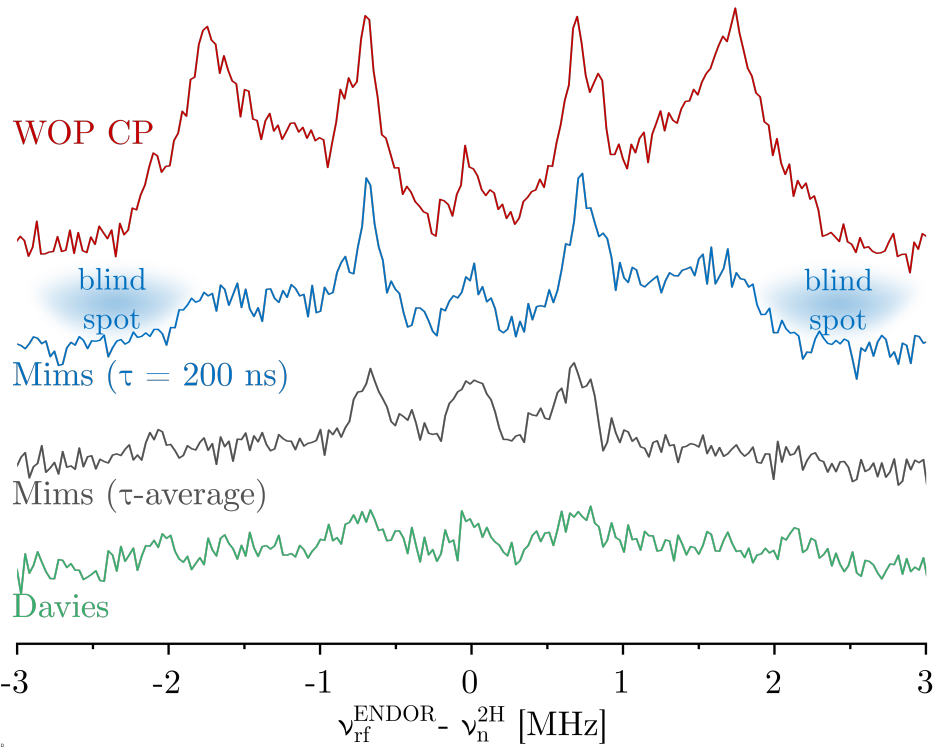

Figure 7.1: ENDOR performance comparison on $\mathrm{ND}_{2} \mathrm{Y}_{731}{ }^{\bullet}$. A) Radical structure of $\mathrm{ND}_{2} \mathrm{Y}_{731} \bullet$ B) $94 \mathrm{GHz}$ field-sweep ESE spectrum of $\mathrm{ND}_{2} \mathrm{Y}_{731}{ }^{\bullet} C$ ) WOP CP-ENDOR (sum of 2D data, red), Mims ENDOR $(\tau=200 \mathrm{~ns}$, blue), Mims ENDOR (sum of $7 \tau$-values: 100, 150, 200, 250, 300, 400, $500 \mathrm{~ns}$, grey) and Davies ENDOR (green), 1800 scans each. Exp. conditions: ESE: $\nu_{\mathrm{EPR}}=94 \mathrm{GHz}, T=70 \mathrm{~K}$, $t_{\pi / 2}=20 \mathrm{~ns}, \tau=240 \mathrm{~ns}$, shot repetition time $=3 \mathrm{~ms}, 50$ shot $/$ point, 10 scans. ENDOR: $T=10 \mathrm{~K}, t_{\pi, \mathrm{rf}}=38 \mu \mathrm{s}, \omega_{1 \mathrm{n}} / 2 \pi=13 \mathrm{kHz}$, shot repetition time $=100 \mathrm{~ms}$, 1 shot/point, random rf acquisition, 1800 scans $(13 \mathrm{~h})$; Davies: $t_{\pi, \text { prep }}=400 \mathrm{~ns}$, $t_{\pi / 2, \mathrm{det}}=20 \mathrm{~ns} ;$ Mims: $t_{\pi / 2, \mathrm{mw}}=20 \mathrm{~ns} ; C P: t_{\pi / 2, \mathrm{det}}=200 \mathrm{~ns}, \omega_{1 \mathrm{e}} / 2 \pi=1.25 \mathrm{MHz}$, $t_{\mathrm{CP}}=200 \mu \mathrm{s}, \Delta \omega_{\mathrm{n}}^{\mathrm{CP}} / 2 \pi=-2.3 \mathrm{MHz}$ to $+2.3 \mathrm{MHz}$ (36 steps).

In order to achieve a representative comparison, Mims and Davies ENDOR experiments were also performed in a (pseudo) 2D fashion. Here, the second dimension is introduced via a "dummy" pulse that has no mw power, but allows for pseudo-incrementation of a $y$-axis. This procedure is necessary for identical Xepr software calculation times which differ by up to $\approx 30 \%$ between a $1 \mathrm{D}$ and $2 \mathrm{D}$ ENDOR experiment. This pseudo-2D approach preserves equal axis-incrementation timings leading to the same relaxation durations for the compared experiments.

The first Mims ENDOR spectrum shows 1800 scans of a fixed $\tau=200$ ns value. Mims blind spots with maximum destructive amplitudes at $\pm 2 \mathrm{MHz}$ (see also Fig. 7.2) are predicted. The second is a sum of 7 Mims ENDOR spectra, each sub-spectrum with 257 scans and $\tau$-values (black) from $100-500 \mathrm{~ns}$, referred to as $\tau$-average. Davies ENDOR 
was recorded with a preparation pulse length of $t_{\pi, \text { prep }}=400 \mathrm{~ns}$ and Hahn-echo detection via $t_{\pi / 2 \text {,det }}=20 \mathrm{~ns}-\tau-t_{\pi, \text { det }}=40 \mathrm{~ns}-\tau$ - echo. The ENDOR rf inversion pulse length was set to $38 \mu \mathrm{s}$ for all experiments.

A first qualitative comparison reveals the same signal-to-noise ratio of WOP CP and Mims ENDOR when considering the peaks at $\pm 0.75 \mathrm{MHz}$. At the peak position, these sequences show factors of 5 and 2 better $\mathrm{S} / \mathrm{N}$ compared to Davies and the $\tau$-average Mims experiments. Additionally, WOP CP-ENDOR reveals broad tensor features above $\pm 0.75 \mathrm{MHz}$, obtained only partially in the Mims ENDOR spectrum.

The unmasked tensor line shapes become more evident from the direct comparison of the previously presented Mims (blue) and WOP CP-ENDOR spectra (red) shown in Fig. 7.2. The spectra are scaled to equal intensities at $\pm 0.75 \mathrm{MHz}$.

WOP CP vs. Mims

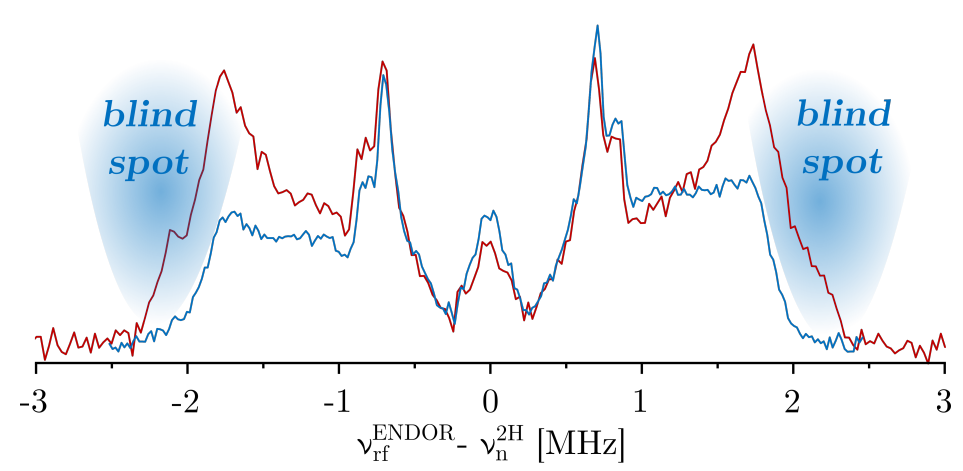

Figure 7.2: Comparison of WOP CP-ENDOR and Mims ENDOR on $\mathrm{ND}_{\mathbf{2}} \mathrm{Y}_{\mathbf{7 3 1}}{ }^{\bullet}$. The WOP CP-ENDOR spectrum (sum of $2 \mathrm{D}$ data, red) of $\mathrm{ND}_{2} \mathrm{Y}_{731}{ }^{\bullet}$ unmasks hyperfine tensor features which are distorted by blind spots in the Mims ENDOR spectrum $\left(\tau=200 \mathrm{~ns}\right.$, blue). Exp. conditions: $T=10 \mathrm{~K}, \nu_{\mathrm{EPR}}=94 \mathrm{GHz}$, $t_{\pi, \mathrm{rf}}=38-45 \mu \mathrm{s}, \omega_{1 \mathrm{n}} / 2 \pi=11-13 \mathrm{kHz}$, shot repetition time $=100 \mathrm{~ms}, 1 \mathrm{shot} /$ point, random rf acquisition; Mims: $t_{\pi / 2, \mathrm{mw}}=20 \mathrm{~ns}, 3680 \mathrm{scans} ; C P: t_{\pi / 2, \mathrm{det}}=200 \mathrm{~ns}$, $\omega_{1 \mathrm{e}} / 2 \pi=1.25 \mathrm{MHz}, t_{\mathrm{CP}}=200 \mu \mathrm{s}, \Delta \omega_{\mathrm{n}}^{\mathrm{CP}} / 2 \pi= \pm 2.3 \mathrm{MHz}(36$ steps $), 3000$ scans. The Mims ENDOR spectrum was recorded during Maik Reinhard's Bachelor thesis ${ }^{105]}$.

A standard procedure to compensate for the blind spots in Mims ENDOR is to record spectra of various $\tau$-values and either sum them up or analyze them individually. The sub-spectra used to obtain the $\tau$-average Mims ENDOR spectrum shown in Fig. 7.1 are plotted in Fig. 7.3. In addition, the blind spot function according to Eq. 2.51 for each case is plotted as dashed lines. It becomes clear, that short $\tau$-values generate a broad blind spot around the nuclear Larmor frequency and that a longer $\tau$-value narrows the blind spot. Additionally, longer $\tau$-values introduce more blind spots on the sides of the spectral range. Overall, none of the $\tau$-values can reproduce the ENDOR spectrum of this radical without artefacts. The $\tau$-average Mims ENDOR spectrum contains all tensor features, but suffers from small $\mathrm{S} / \mathrm{N}$. 
Mims-ENDOR at $B_{0} / g_{y}$

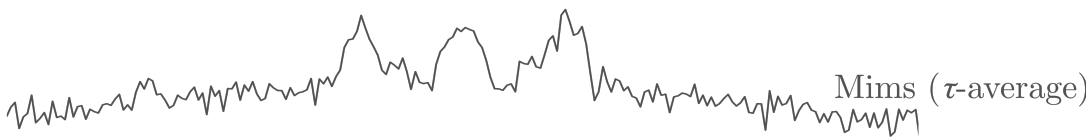

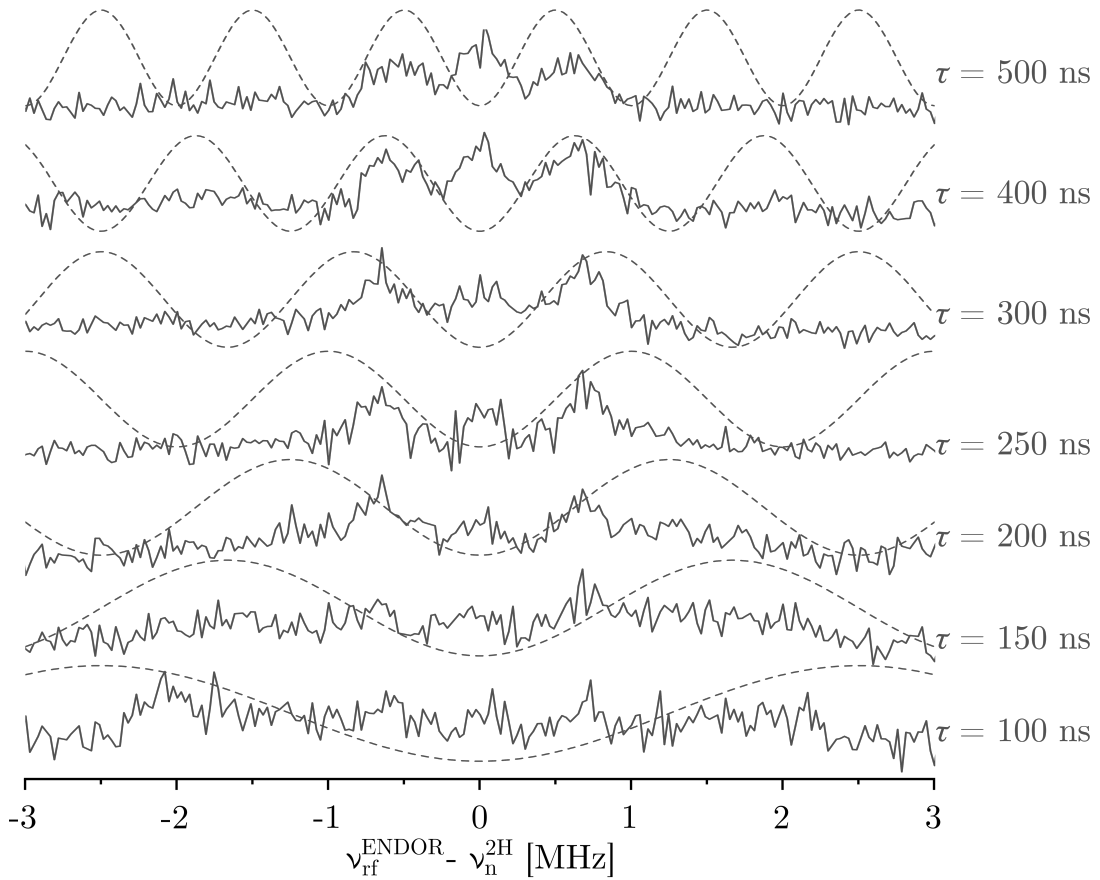

Figure 7.3: $\tau$-averaged Mims ENDOR of $\mathrm{ND}_{\mathbf{2}} \mathrm{Y}_{\mathbf{7 3 1}}{ }^{\bullet}$. Spectra were recorded for seven $\tau$-values: 100, 150, 200, 250,300, 400, 500 ns. Sub-spectra of individual $\tau$-values and corresponding Mims hole function (dotted lines) are shown. Exp. conditions: $T=10 \mathrm{~K}, \nu_{\mathrm{EPR}}=94 \mathrm{GHz}, t_{\pi, \mathrm{rf}}=38 \mu \mathrm{s}, \omega_{1 \mathrm{n}} / 2 \pi=13 \mathrm{kHz}$, shot repetition time $=100 \mathrm{~ms}, 1$ shot $/$ point, random $\mathrm{rf}$ acquisition, $t_{\pi / 2, \mathrm{mw}}=20 \mathrm{~ns}$, 1800 scans ( $\tau$-average); 257 scans $(\tau$-sub-spectra).

\subsubsection{Determination of the $\mathrm{ND}_{2}$-conformation from WOP $\mathrm{CP}$ - ENDOR}

In order to determine the conformation of the amino group relative to the tyrosyl ring plane, orientation-selective WOP CP-ENDOR experiments were performed. The landscapes of the 2D WOP CP-ENDOR experiments are shown in Fig. 7.4 and the resulting ENDOR spectra and simulations are summarized in Fig. 7.5. The simulations were performed with an in-house routine for the simulation of conventional ENDOR spectra ${ }^{[13]}$. In the following, such simulations will be referred to as Tensor simulation and will be later compared to the $\mathrm{CP}$ simulation approach. 
A) $2 D$ WOP CP-ENDOR at $B_{0} / / g_{x}$

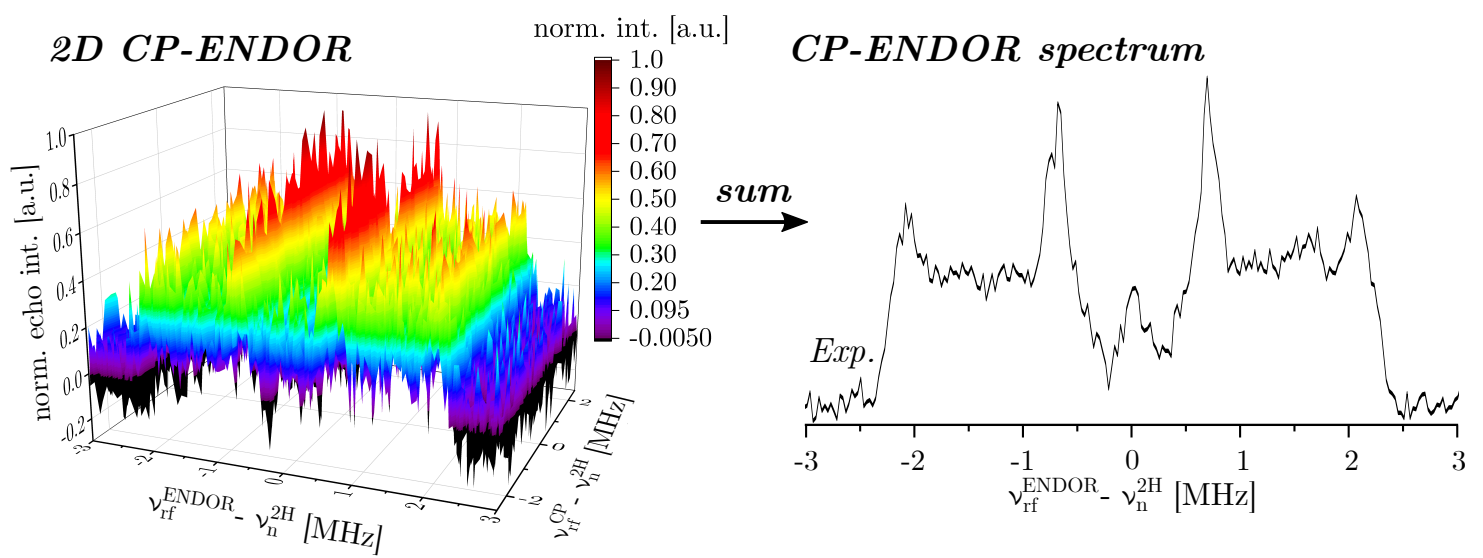

B) $2 D$ WOP CP-ENDOR at $B_{0} / / g_{y}$

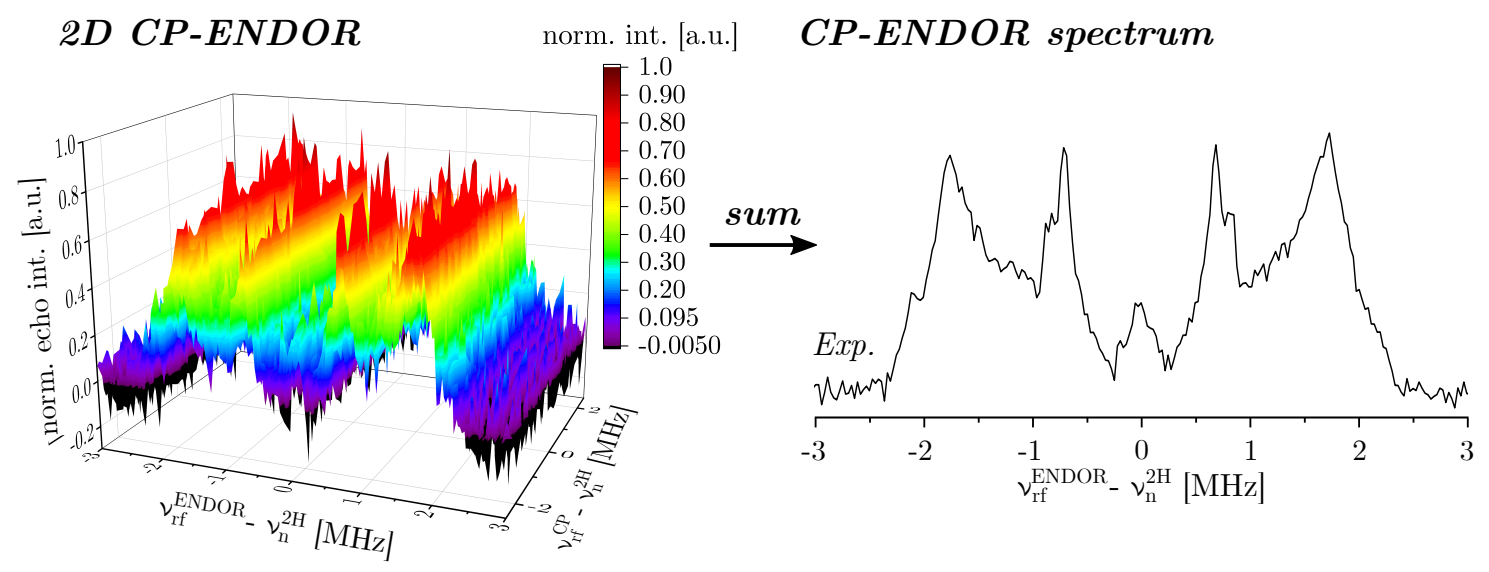

C) $2 D$ WOP CP-ENDOR at $B_{0} / / g_{z}$

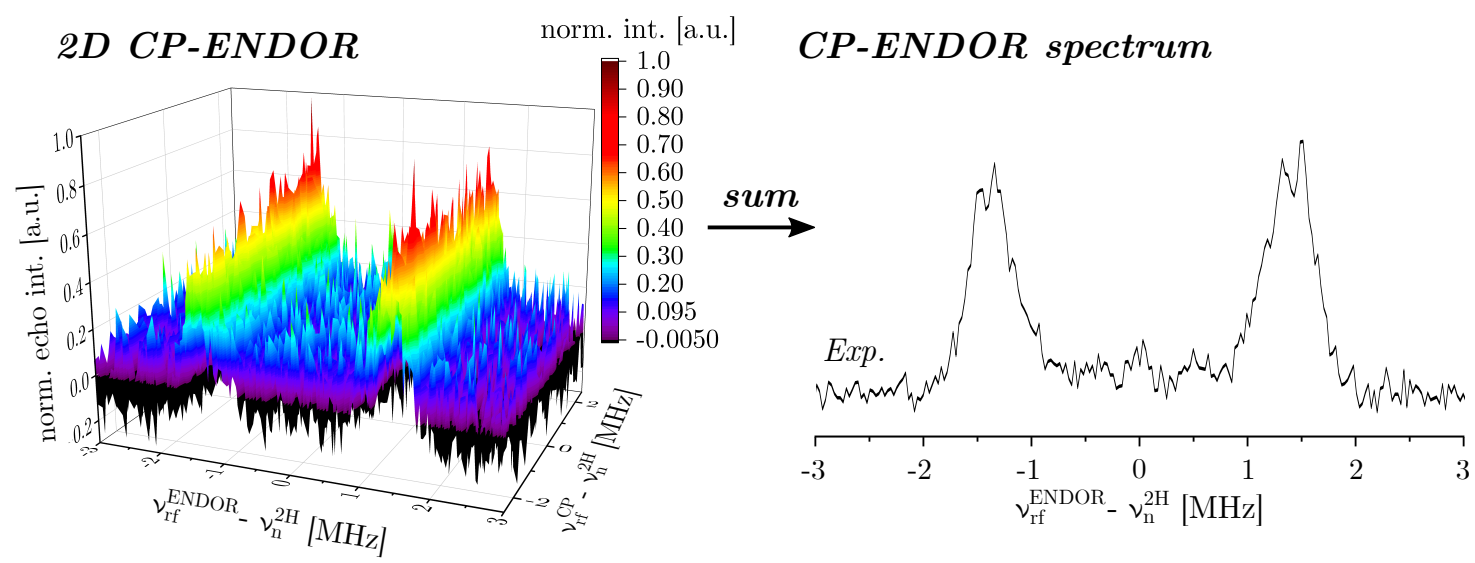

Figure 7.4: Landscape plots of orientation-selective 2D WOP CP-ENDOR experiments of $\mathbf{N D}_{\mathbf{2}} \mathbf{Y}_{\mathbf{7 3 1}} \bullet$. Spectra were recorded at $B_{0}\left\|g_{x}(\mathrm{~A}), B_{0}\right\| g_{y}(\mathrm{~B})$ and $B_{0} \| g_{z}(\mathrm{C})$. The ENDOR spectra shown in the right column were obtained from summation of the $2 \mathrm{D}$ data set. Exp. conditions: $T=10 \mathrm{~K}, \omega_{1 \mathrm{e}} / 2 \pi=1.25 \mathrm{MHz}, \omega_{1 \mathrm{n}} / 2 \pi=13 \mathrm{kHz}, t_{\pi / 2 \text {,det }}=200 \mathrm{~ns}$, $t_{\mathrm{CP}}=200 \mu \mathrm{s}, \Delta \omega_{\mathrm{n}}^{\mathrm{CP}} / 2 \pi=-2.3 \mathrm{MHz}$ to $+2.3 \mathrm{MHz}$ (36 steps), $3000-5000$ scans. 
A) WOP CP-ENDOR at $B_{0} / / g_{x}$

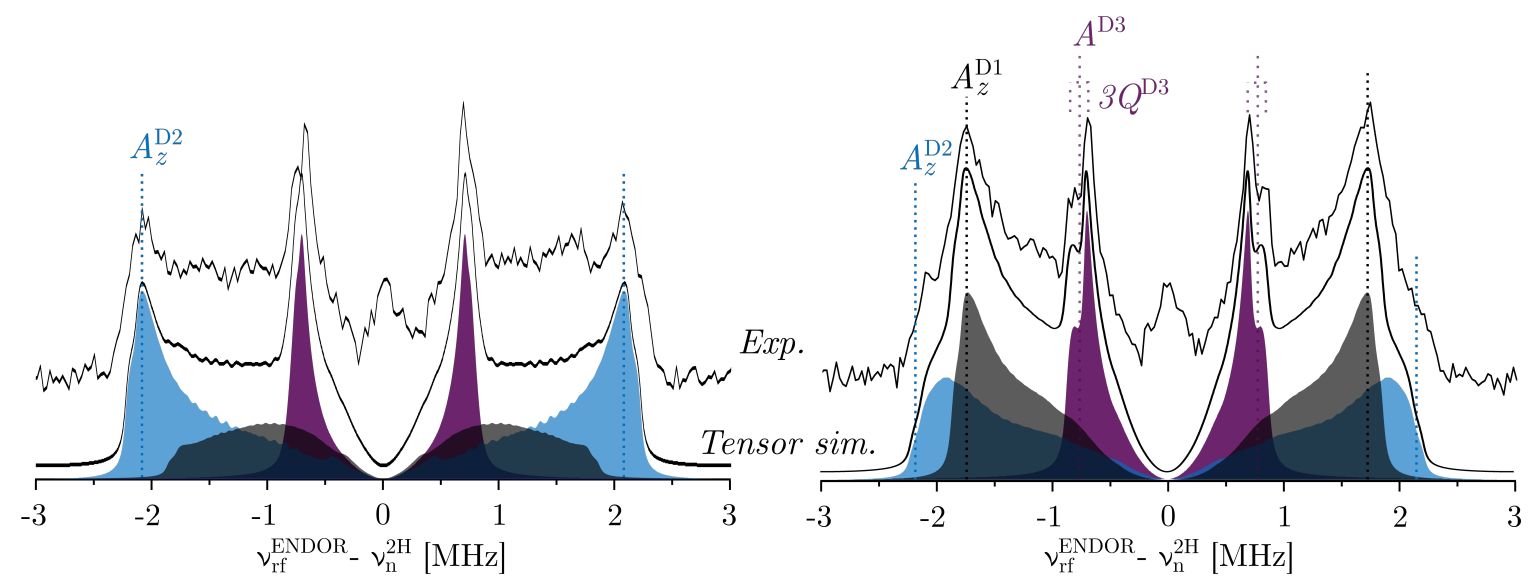

C) WOP CP-ENDOR at $B_{0} / / g_{z}$

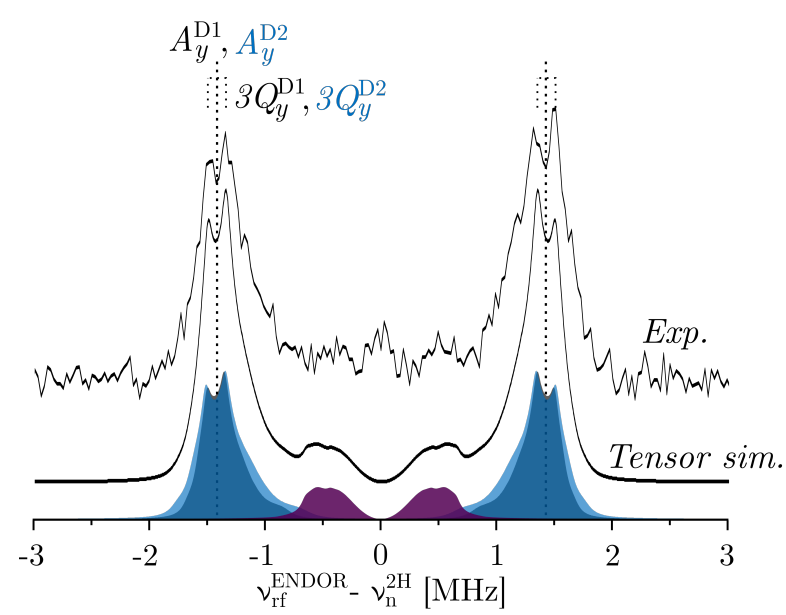

D)
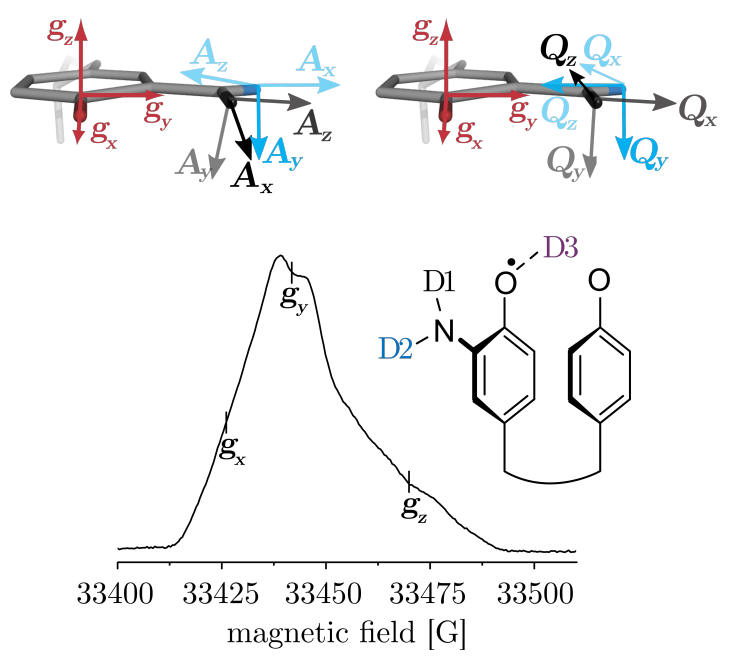

Figure 7.5: Orientation-selective WOP CP-ENDOR experiments and simulations of $\mathbf{N D}_{\mathbf{2}} \mathbf{Y}_{\mathbf{7 3 1}}{ }^{\bullet}$. ENDOR spectra were recorded on $B_{0}\left\|g_{x}(A), B_{0}\right\| g_{y}$ $(B)$ and $B_{0} \| g_{z}(C)$. The simulation includes couplings of two amino deuterons (gray and blue) and one perpendicular H-bond (purple) according to parameters listed in Table 7.1. D) $94 \mathrm{GHz}$ ESE-detected field sweep spectrum of $\mathrm{ND}_{2} \mathrm{Y}_{731} \bullet$ and deuterium hf- and quad-tensor orientations obtained from DFT predictions ${ }^{[17]}$ (planar $\mathrm{ND}_{2}$ ). Coupling parameters and Euler angles are listed in Table 7.1. Experimental parameters can be found in the captions of Fig. 7.8 (ENDOR) and Fig. 7.1 (ESE).

In order to obtain the simulations shown in Fig. 7.5, the DFT predicted tensor values and orientations ${ }^{17]}$ (depicted in Fig. 7.5, D) were examined and adjusted as discussed in the following. The parameters are summarized in Table 7.1. In addition to the ENDOR simulation (Sim.), the individual hf-tensor contributions of both amino deuterons (gray and blue) and the perpendicular H-bond (purple) were plotted. Characteristic tensor features of both amino deuteron hfcs (gray and blue) are marked in each spectrum. The hf coupling $A$ (D3) to the H-bond (purple) at around $\pm 0.75 \mathrm{MHz}$ is not in the focus of this thesis, but has been discussed elsewhere ${ }^{[17,18}$. In general, the ENDOR 
spectra at $B_{0} \| g_{y}$ and $B_{0} \| g_{x}$ show broad ranges of deuterium resonances, while the ENDOR spectrum at $B_{0} \| g_{z}$ reveals narrow tensor features. In particular, at $B_{0} \| g_{x}$ the maximum $4^{4}$ hf-tensor value of the amino deuterons D2 is observed with only minor contributions of D1 (gray). Thus at this $g$-tensor orientation, $A_{z}(\mathrm{D} 1)$ was considered almost collinear with $A_{x}(\mathrm{D} 2)$. Finally, narrow tensor features at $B_{0} \| g_{z}$ indicate that the tensor components of both deuterons are almost collinear with each other and collinear with the $g_{z}$ principle axis $\left(g_{z}\left\|A_{y}(\mathrm{D} 1)\right\| A_{y}(\mathrm{D} 2)\right)$. Likewise, the quadrupole splittings $Q_{z}(\mathrm{D} 1)=Q_{z}(\mathrm{D} 2)$ are identical in size and tensor orientation (see also Fig. 7.5, D). The described mutual tensor orientations can be satisfied in a planar configuration, as predicted by the DFT calculation ${ }^{17}$.

Table 7.1: Hyperfine tensor values, quadrupole tensor values and corresponding Euler angles for $\mathrm{ND}_{\mathbf{2}} \mathrm{Y}_{\mathbf{7 3 1}}{ }^{\circ}$. Optimized parameters for ENDOR simulation in the first column of each row, DFT parameters (model 3 from ref ${ }^{[17]}$ ) in round brackets (second row) and from ${ }^{1} \mathrm{H}$ ENDOR and ESE-detected field sweep experiments $[86$ in square brackets (third row). DFT uncertainty for hfcs and Euler angles is estimated to be $10 \%$.

\begin{tabular}{|c|c|c|c|c|c|c|c|c|c|c|c|c|}
\hline \multirow[b]{2}{*}{ Nuclei } & \multicolumn{3}{|c|}{$\begin{array}{l}\text { Hyperfine coupling } \\
{[\mathrm{MHz}]}\end{array}$} & \multicolumn{3}{|c|}{$\begin{array}{c}\text { Euler angles } \\
\text { for } \mathrm{hfc}\left[^{\circ}\right]\end{array}$} & \multicolumn{3}{|c|}{$\begin{array}{l}\text { Quad. coupling } \\
{[\mathrm{MHz}]}\end{array}$} & \multicolumn{3}{|c|}{$\begin{array}{c}\text { Euler angles for } \\
\text { quad. couplings }\left[^{\circ}\right]\end{array}$} \\
\hline & $A_{x}$ & $A_{y}$ & $A_{z}$ & $\alpha$ & $\beta$ & $\gamma$ & $Q_{x}$ & $Q_{y}$ & $Q_{z}$ & $\alpha$ & $\beta$ & $\gamma$ \\
\hline D1 & 0.47 & -2.85 & -3.65 & -98 & 99 & 82 & -0.03 & -0.05 & 0.08 & -95 & 80 & -15 \\
\hline (Amino & $(0.47)$ & $(-2.6)$ & $(-3.4)$ & $(-98)$ & (99) & $(72)$ & $(-0.05)$ & $(-0.06)$ & $(0.11)$ & $(-95)$ & (80) & $(-15)$ \\
\hline deuteron 1) & {$[0.46]$} & {$[-2.77]$} & {$[-3.1]$} & {$[-86]$} & [98] & [90] & & & & & & \\
\hline D2 & 0.67 & -2.85 & -4.35 & -100 & 94 & -48 & -0.03 & -0.06 & 0.09 & -98 & 93 & 43 \\
\hline (Amino & $(0.67)$ & $(-2.63)$ & $(-3.97)$ & $(-100)$ & (84) & $(-45)$ & $(-0.05)$ & $(-0.06)$ & $(0.11)$ & $(-98)$ & (103) & $(43)$ \\
\hline deuteron 2) & {$[0.77]$} & {$[-2.77]$} & {$[-3.7]$} & {$[-96]$} & [93] & {$[-31]$} & & & & & & \\
\hline D3 & 1.1 & -1.45 & -1.59 & -160 & 120 & 80 & -0.04 & -0.06 & 0.1 & 106 & 35 & 95 \\
\hline (perp. & (1.1) & $(-1.7)$ & $(-2)$ & $(-163)$ & (122) & (81) & $(-0.04)$ & $(-0.06)$ & $(0.09)$ & (106) & (25) & $(84)$ \\
\hline H-bond) & {$[1.1]$} & {$[-1.2]$} & {$[-1.3]$} & {$[-160]$} & [110] & {$[80]$} & & & & & & \\
\hline
\end{tabular}

In the following, the uncertainty of the validated planar configuration is determined by comparing the WOP CP-ENDOR experiments with a series of simulations in which the structure of the functional group is modified. For a quantitative analysis of the obtained simulations, the normalized root-mean-square deviation (NRMSD) of the simulations from the experimental spectra was calculated. The results are summarized in Fig. 7.6. The conformational change is rationalized by introducing the tilt angle $\phi$, which is defined as angle between the plane spanned by the directions of the $g$-tensor principal axes $g_{x}$ and $g_{y}$ (green plane in Fig. 7.6, C) and the D-N-D-spanned plane (red in Fig. 7.6, C).

\footnotetext{
${ }^{4}$ In this context minimum/small and maximum/large refer to the absolute hfc values.
} 
ENDOR Tensor simulations

E) $B_{0} / g_{x}$

A)

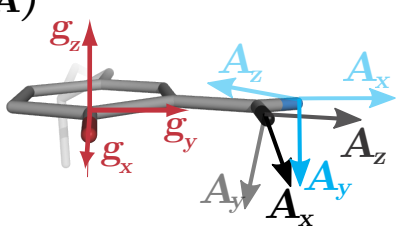

planar, $\phi=0^{\circ}$

C)

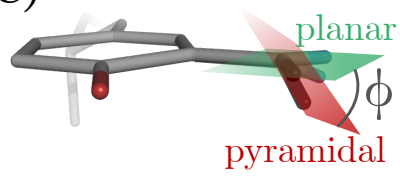

B)

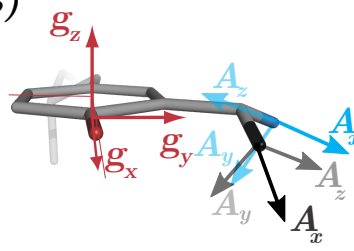

pyramidal, $\phi=50^{\circ}$

D)

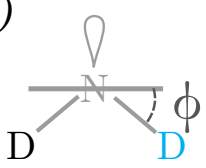

F) Average normalized RMSD

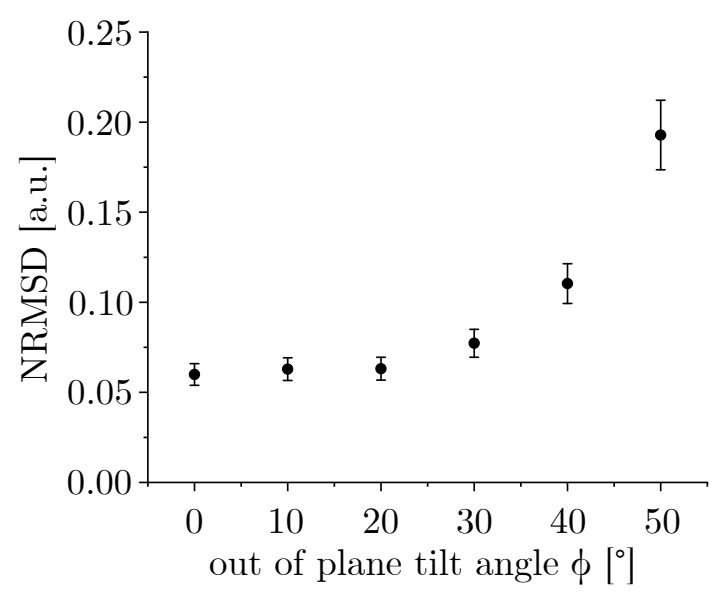

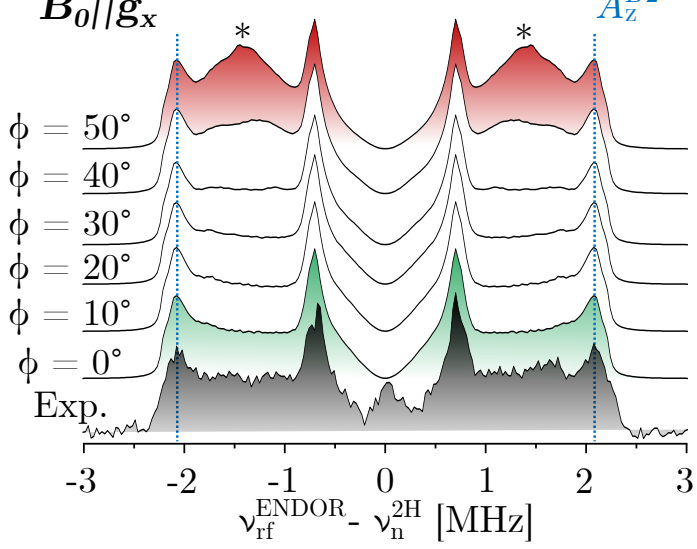
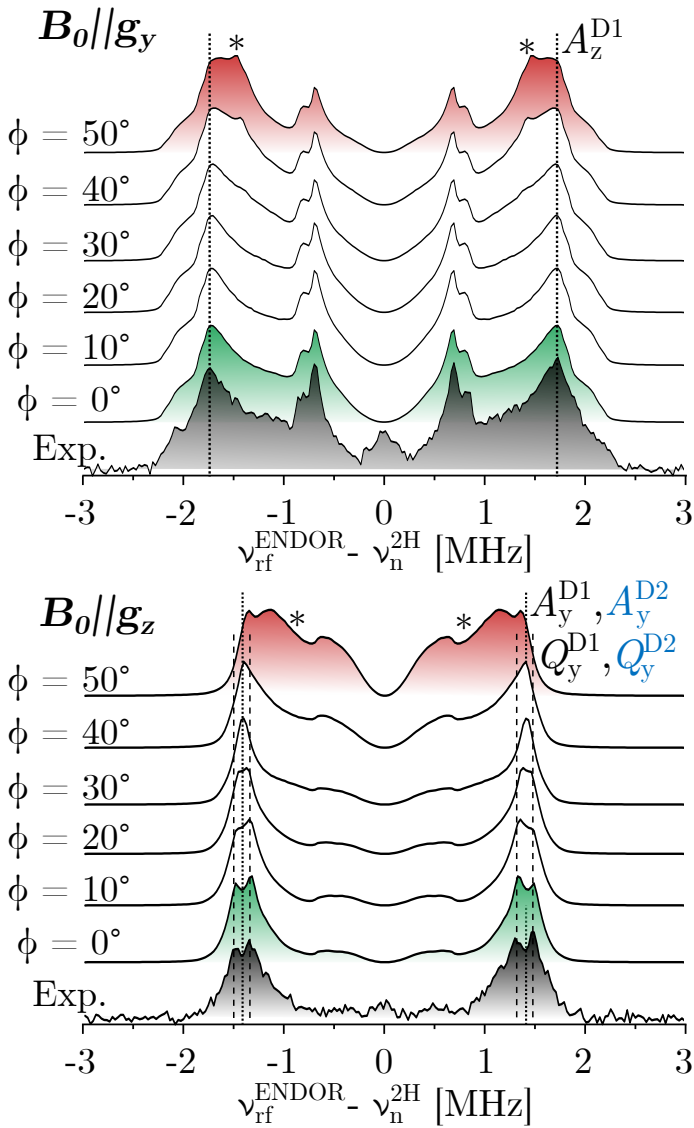

Figure 7.6: Systematic ENDOR simulations for conformational changes of $\mathbf{N D}_{2} \cdot A-D$ ) Illustration of $g$ - and $A$-tensor orientations for planar (green plane) and pyramidal (red plane) configuration. E) Systematic ENDOR Tensor simulations for $\phi=0$ to $50^{\circ}$ at $B_{0} \| g_{x}, g_{y}, g_{z}$. The asterisks mark the most characteristic, tensor features introduced by variation of $\phi . F$ ) Normalized root-mean-square deviation (NRMSD) vs. tilt angle plot on the basis of ENDOR simulations shown in $E$. The NRMSD is obtained from averaging the RMSD calculated for $B_{0} \| g_{x}, g_{y}, g_{z}$ for each $\phi$ value. The uncertainty is estimated to be 10\%. Coupling values and Euler angles are listed in Table 7.1. The tilt angle $\phi$ is encoded in the first Euler rotation $\phi=|\alpha|-90^{\circ}$, thus $\alpha$ was incremented in $10^{\circ}$ steps. 
For $\phi=0^{\circ}$, both planes coincide and the amino-configuration is called planar (Fig. 7.6, A,C). Successive increase of $\phi$ leads to a pyramidal structure $\phi=50^{\circ}$ (Fig. 7.6, C). This tilting angle $\phi$ is linked to the rotation matrix $R_{z y z}=R_{z}(\gamma) R_{y}(\beta) R_{z}(\alpha)$, which defines a rotation around axes $z, y^{\prime}, z^{\prime \prime}$ (in that order) by Euler angles $\alpha, \beta, \gamma$ which connect the $A$ with the $g$-tensor principal axis system in the simulation routine. Euler angles are defined according to magnetic resonance convention in the right-handed coordinate systems for counterclockwise rotations. Finally, the angle $\phi$ is encoded in the first Euler rotation $Z(\alpha)$ or $\phi=|\alpha|-90^{\circ}$ because the direction of the largest hfc component was predicted in the C-N-D plane $\left(A_{z}\right)$. The ND-bond direction is defined to coincide with the smallest hfc tensor $A_{x}$ (Fig. 7.6, A). Furthermore, the ND-bond direction is defined to coincide with the largest quadrupole tensor value $Q_{z}$ (see Fig. 7.5, D). For simplicity, the $A_{x, y, z}$ and $Q_{x, y, z}$ values were kept constant. The simplification was introduced on the basis of DFT calculations of the aminophenol ${ }^{86}$, which have predicted a minor change $(\sim 10 \%)$ of the isotropic ${ }^{1} \mathrm{H}-\mathrm{NH}$ hfcs by variation of $\phi$. Even though the calculation was done for protons, meaning that the quadrupole coupling cannot be predicted, only a minor deviation of the isotropic quadrupole couplings by variation of $\phi$ was assumed.

The consequences for the hf principal axis orientation in the planar and pyramidal case are as follows: Concluded from the narrow ENDOR spectrum at $B_{0} \| g_{z}$, a planar configuration $\left(\phi=0^{\circ}\right)$ restricts the $A_{y}$ and $Q_{y}$ principal axis of both deuterons to be almost collinear with each other and collinear with the $g_{z}$ axis $\left(g_{z}\left\|A_{y}(\mathrm{D} 1)\right\| A_{y}(\mathrm{D} 2)\right.$ $\left.\left\|Q_{y}(\mathrm{D} 1)\right\| Q_{y}(\mathrm{D} 2)\right)$ (Fig. 7.5, D). For increasing $\phi$, the ENDOR spectrum at $B_{0} \| g_{z}$ forfeits the clear quadrupole splitting and the tensor line shape becomes significantly broader. Indeed, variation of $\phi$ breaks the collinearity (Fig. 7.6, B) and results a wider distribution of ENDOR features, as obtained for $\phi=50^{\circ}$ (asterisk in Fig. 7.6, E, bottom). Increasing $\phi$ introduces additional tensor features (Fig. 7.6, E, middle and top) into the respective ENDOR spectrum at $B_{0} \| g_{x}$ and $B_{0} \| g_{y}$. These are marked with an asterisks and assigned to the $A_{y}(\mathrm{D} 1)$ and $A_{y}(\mathrm{D} 2)$ component at $B_{0} \| g_{x}$ and $B_{0} \| g_{y}$, respectively. Finally, the average deviation of the simulation from the experiment was calculated for a tilt angle $\phi$ at each canonical orientation $g_{x}, g_{y}$ and $g_{z}$ (NRMSD). The simulation for $\phi=0-20^{\circ}$ is in good agreement with the experiment. A moderate deviation is obtained for $\phi=30^{\circ}$ which increases by a factor of 2 and 4 for $\phi=40^{\circ}$ and $\phi=50^{\circ}$, respectively. 


\subsubsection{Simulation of orientation-selective 2D WOP CP-ENDOR spectra}

In addition to the simulations presented in the preceding section, the introduced CPENDOR routine developed in this thesis was extended to orientation-selective 2D WOP CP-ENDOR spectra. The second dimension of the 2D CP-ENDOR experiment is not predictable by the Tensor simulation routine used for the above $\mathrm{ND}_{2}$ tensor analysis. The Tensor simulation approach for the simulation of conventional Mims and Davies ENDOR spectra predicts ENDOR resonances according to the analytically derived resonance conditions (Eq. 2.34). Indeed, the simulations gathered for the analysis of the $\mathrm{ND}_{2}$-conformation were based on such a Tensor simulation script. For the simulation of WOP CP-ENDOR spectra, a blind spot around $\mathrm{A}=0 \mathrm{MHz}$ was introduced by multiplication with a blind spot function $\left(1-\frac{c}{x^{2}-c}\right)$ centered at the Larmor frequency of the ENDOR spectrum. The factor $c$ was chosen to match the simulated relative intensities to the experimental intensities. In the following, this approach will be referred to as Tensor simulation. In order to simulate orientation-selective 2D WOP CP-ENDOR spectra (i.e. landscapes shown in Fig 7.4), two extensions of the CP simulation were introduced: (a) three coupled nuclei and (b) orientation selection. The procedure is described in Subsec. 4.3.3. The simulations obtained from this approach will be referred to as $C P$ simulation.

The simulated 2D WOP CP-ENDOR spectra are shown in the right column of Fig. 7.7 together with the corresponding experimental results in the left column. All spectra were individually normalized to their maximum intensity. A summary of the simulation parameters can be found in the figure caption, while hf and quad coupling values and Euler angles are summarized in Table 7.1.

The number of $x$-axis points in the simulation is half of the experimental dimension (256 vs. 128 points). This shortened the calculation time to 4-6 weeks for each simulation and allowed for several optimization steps. Besides the ENDOR/EPR parameters, also mw and rf amplitudes were carefully optimized. Among other aspects, the mw amplitude determines the width of the central blind spot and the rf amplitude scales the ENDOR line broadening. Finally, it is noted that the $C P$ simulation is free from an additional line shape modification which was needed to consider the central blind spot of the ENDOR spectrum in the Tensor simulation. Overall, a good qualitative agreement between experimental and simulated 2D WOP CP-ENDOR spectra was obtained. However a 
quantitative analysis was not included due to the limited $\mathrm{S} / \mathrm{N}$ of the individual $\mathrm{CP}$ landscapes' slices.

A) $2 D$ WOP CP-ENDOR at $B_{0} / / g_{x}$

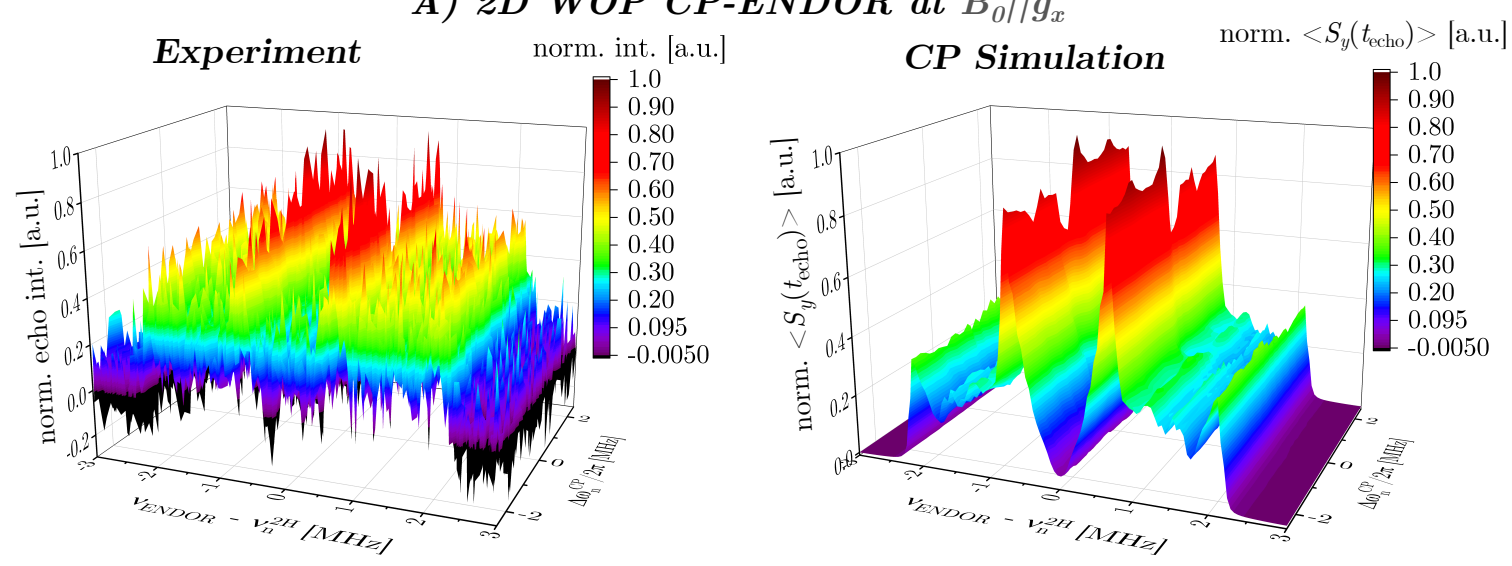

B) $2 D$ WOP CP-ENDOR at $B_{0} / / g_{y}$
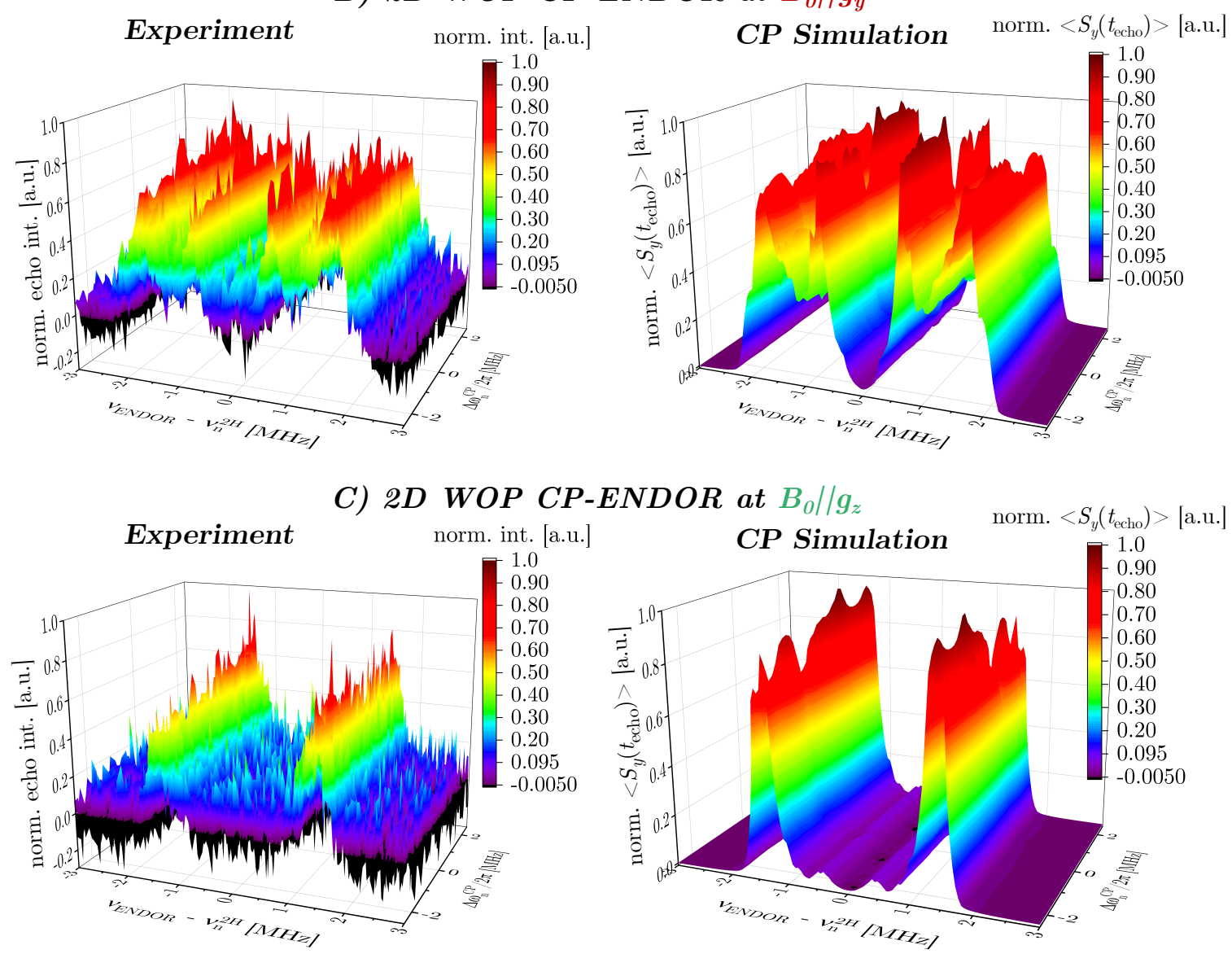

Figure 7.7: Orientation-selective 2D WOP CP-ENDOR experiments and simulations of $\mathbf{N D}_{\mathbf{2}} \mathbf{Y}_{\mathbf{7 3 1}}{ }^{\bullet}$. Spectra are shown for $B_{0}\left\|g_{x}(\mathrm{~A}), B_{0}\right\| g_{y}(\mathrm{~B})$ and $B_{0} \| g_{z}$ (C). CP sim.: $\omega_{1 \mathrm{e}} / 2 \pi=1.25 \mathrm{MHz}, \omega_{1 \mathrm{n}} / 2 \pi=13 \mathrm{kHz}, t_{\pi / 2 \text {,det }}=200 \mathrm{~ns}, t_{\mathrm{CP}}=200 \mu \mathrm{s}$, $\Delta \omega_{\mathrm{n}}^{\mathrm{CP}} / 2 \pi=-2.3 \mathrm{MHz}$ to $+2.3 \mathrm{MHz}$ (36 steps), $N_{\theta}=N_{\phi}=162$. Hfcs and Euler angles are listed in Table 7.1. Exp. conditions: $T=10 \mathrm{~K}, \omega_{1 \mathrm{e}} / 2 \pi=1.25 \mathrm{MHz}$, $\omega_{1 \mathrm{n}} / 2 \pi=13 \mathrm{kHz}, t_{\pi / 2, \mathrm{det}}=200 \mathrm{~ns}, t_{\mathrm{CP}}=200 \mu \mathrm{s}, \Delta \omega_{\mathrm{n}}^{\mathrm{CP}} / 2 \pi=-2.3 \mathrm{MHz}$ to $+2.3 \mathrm{MHz}$ (36 steps), 3000-5000 scans. 
The simulated 2D WOP CP-ENDOR spectra were summed up, similar to the experimental approach, in order to obtain the WOP CP-ENDOR spectrum. In Fig. 7.8 the resulting WOP CP-ENDOR simulations are compared to the experimental ENDOR spectra and to the corresponding Tensor simulation. Considering the different $x$-axis resolution (half of the resolution in $C P$ simulation), the spectra obtained from the $C P$ simulation and Tensor simulation approach are in good agreement with each other and also with the experiment.

A) WOP CP-ENDOR at $B_{0} / / g_{x}$

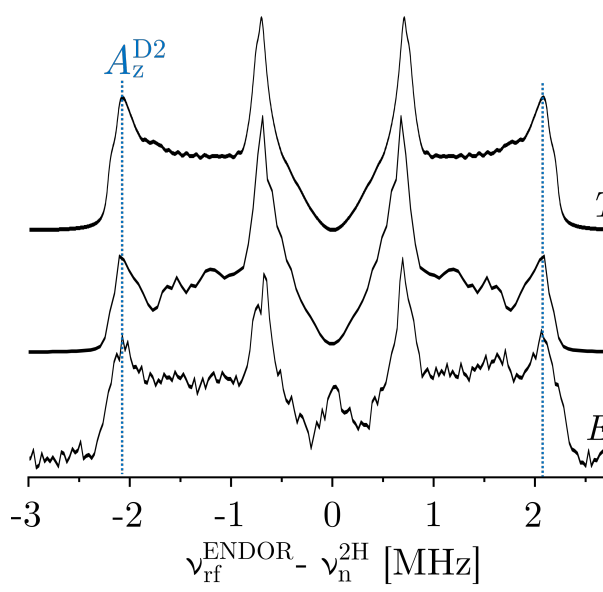

C) WOP CP-ENDOR at $B_{0} / / g_{z}$

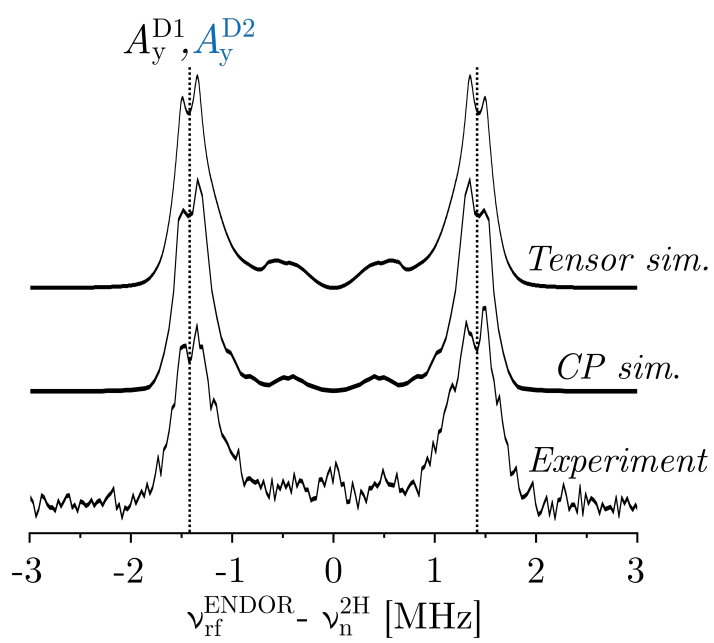

B) WOP CP-ENDOR at $B_{0} / / g_{y}$

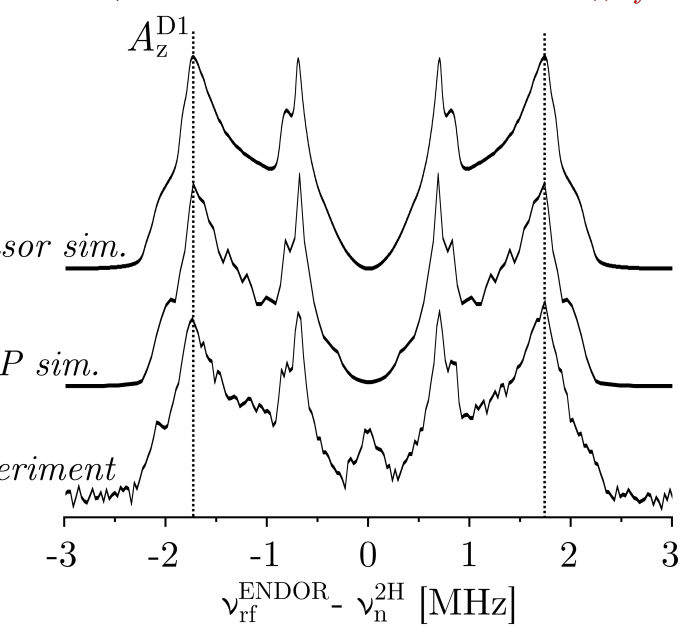

D) ESE-detected field sweep spectrum

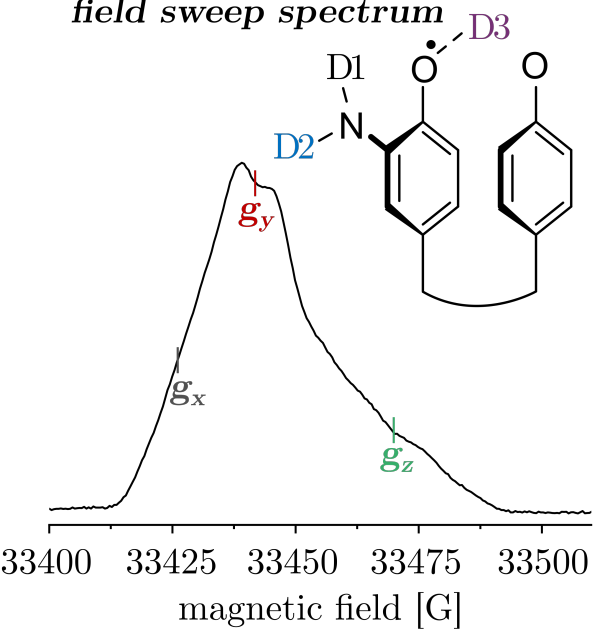

Figure 7.8: Comparison of experiments and two simulation approaches for orientation-selective WOP CP-ENDOR of $\mathrm{ND}_{\mathbf{2}} \mathrm{Y}_{\mathbf{7 3 1}}{ }^{\bullet} \cdot A-C$ ) Orientationselective WOP CP-ENDOR spectra and simulations of $\mathrm{ND}_{2} \mathrm{Y}_{731} \bullet$. Two simulation approaches are exploited: A well-established tensor simulation (Tensor sim.) and the CP-ENDOR simulation ( $C P$ sim.) obtained from summation of the simulated 2D landscapes shown in Fig. 7.7. D) $94 \mathrm{GHz}$ ESE-detected field sweep spectrum of $\mathrm{ND}_{2} \mathrm{Y}_{731}$. Exp. and sim. parameters can be found the caption of Fig. 7.7 . 
In general, the WOP CP-ENDOR spectrum (without CP information) can be obtained from simulating spinlock ENDOR only. In recapitulation, the WOP CP-ENDOR sequence without rf irradiation simultaneously to the mw irradiation is referred to as spinlock ENDOR. The spinlock ENDOR simulation relies on the same simulation script as the CP simulation, but the rf amplitude during spinlock is set to $0 \mathrm{kHz}$ and only a single spinlock ENDOR spectrum is calculated. Therefore, the spinlock ENDOR simulation requires only a fraction of calculation time (1-3 days) compared to the full 2D WOP CP-ENDOR (4-6 weeks). In Fig. 7.9 the simulated spinlock ENDOR spectrum is compared to the calculated WOP CP-ENDOR spectrum (summation of 2D WOP CP-ENDOR). The obtained tensor line shapes are identical, which validates that summation of the 2D WOP CP-ENDOR results in reliable ENDOR spectra without introducing distortions due to for instance lopsided CP enhancement of hfcs.
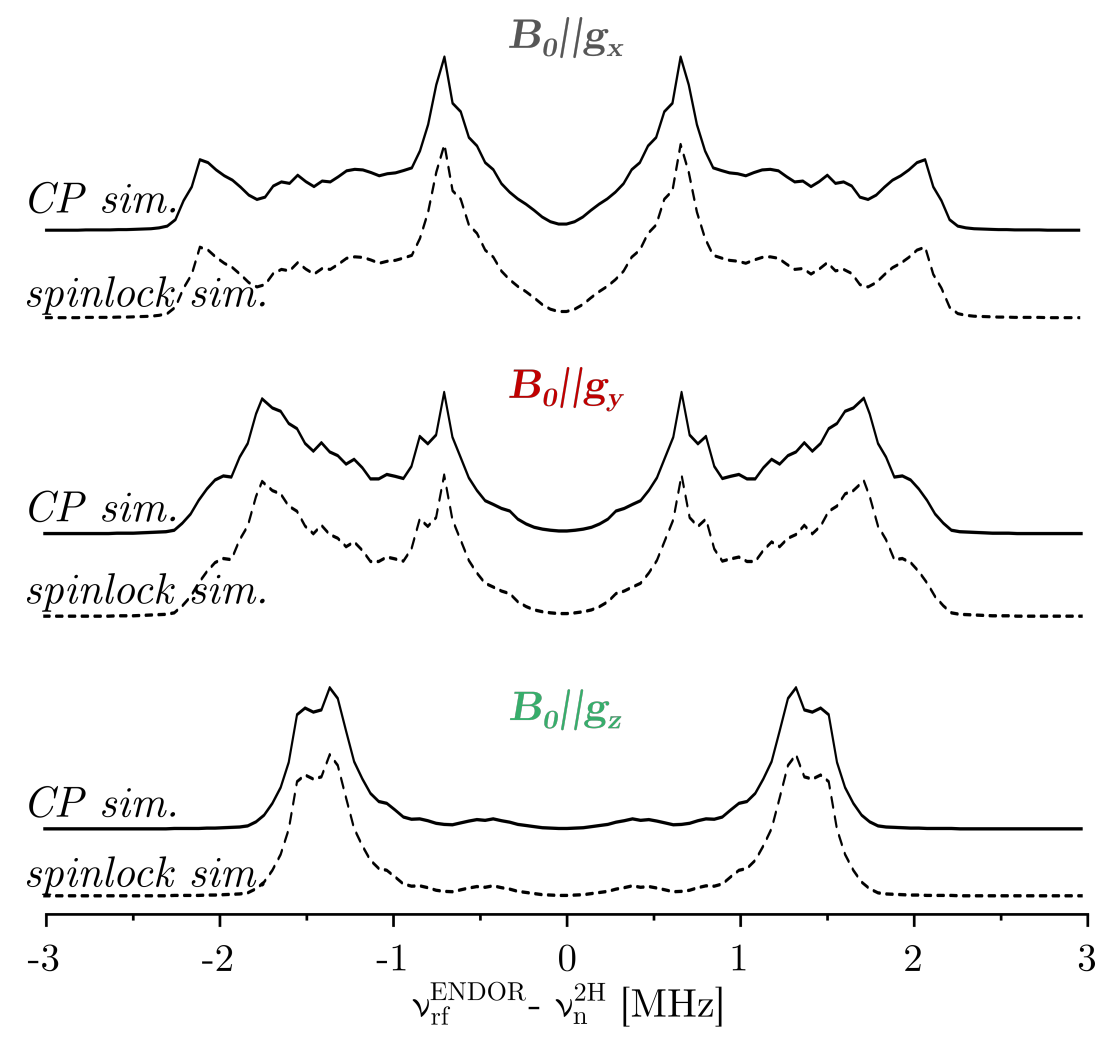

Figure 7.9: Orientation-selective WOP CP-ENDOR and spinlock ENDOR simulation of $\mathrm{ND}_{\mathbf{2}} \mathrm{Y}_{\mathbf{7 3 1}}{ }^{\bullet}$. CP sim. refers to simulation and summation of the full $2 \mathrm{D}$ data set. The spinlock sim. relates to the same simulation script, but without rf matching during the CP step and only a single spinlock ENDOR spectrum is calculated.

Finally, the robustness of the CP simulation/spinlock ENDOR simulation is demonstrated by a similar investigation of the $\mathrm{ND}_{2}$-conformation previously shown for the Tensor simulation in Fig. 7.6. The resulting spinlock ENDOR simulations are displayed in Fig. 7.10. 
ENDOR spinlock simulations

A)

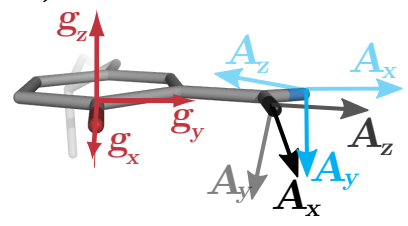

planar, $\phi=0^{\circ}$

C)

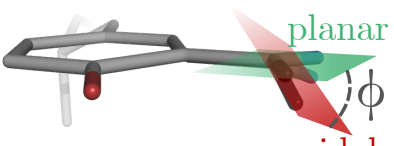

pyramidal
B)

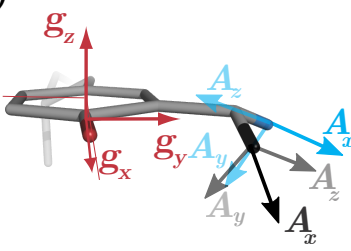

pyramidal, $\phi=50^{\circ}$

D)

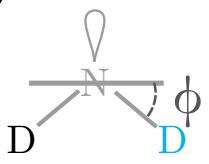

E)
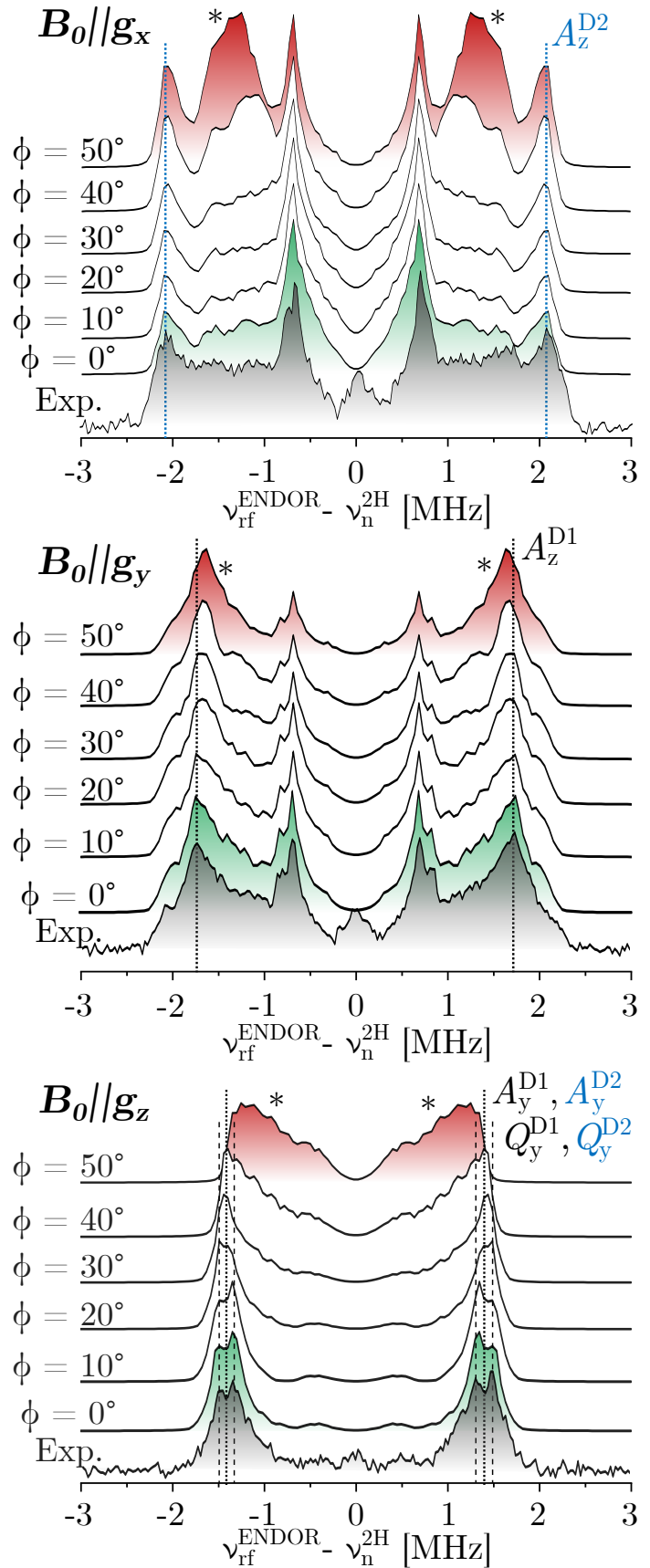

Figure 7.10: Systematic spinlock ENDOR simulations for conformational changes of $\left.\mathbf{N D}_{2} A-D\right)$ Illustration of $g$ - and $A$-tensor orientations for planar and pyramidal configuration. E) Systematic spinlock ENDOR simulations for $\phi=0$ to $50^{\circ}$ at $B_{0} \| g_{x}, g_{y}, g_{z}$. The asterisks mark the most characteristic, tensor features introduced by variation of $\phi . F$ ) Normalized root-mean-square deviation (NRMSD) dependency on $\phi$ calculated on the basis of spinlock ENDOR simulations shown in $E$. The NRMSD is obtained from averaging the RMSD for each $\phi$ value at $B_{0} \| g_{x}, g_{y}, g_{z}$. The uncertainty is estimated to be $10 \%$. The CP spinlock simulation parameters are listed in the caption of Fig. 7.7. Hfcs and Euler angles are listed in Table 7.1. The tilt angle $\phi$ is encoded in the first Euler rotation $\phi=|\alpha|-90^{\circ}$, thus $\alpha$ was incremented in $10^{\circ}$ steps. 
The spinlock ENDOR simulation was used to examine the change of the ENDOR line shape by variation of $\phi$. The analysis on the basis of the spinlock ENDOR simulation was chosen over the $C P$ simulation because the crucial information is gained from the ENDOR line shape irrespective of the CP effect. For clarification, the CP simulation will predict the same ENDOR line shape as demonstrated in Fig. 7.9, but requires an unprofitable calculational overhead. The simulated spinlock ENDOR spectra are in good agreement with the results of the Tensor simulation approach (compare Fig. 7.10 with Fig. 7.6 ). Ultimately, the results obtained from the spinlock ENDOR simulations likewise establish planarity of the functional group.

\subsection{Discussion of WOP CP-ENDOR on $\mathrm{ND}_{2} \mathrm{Y}_{731^{\circ}}$}

\subsubsection{WOP CP-ENDOR performance and orientation-selective CP-ENDOR simulations}

The comparison of the Mims, Davies and CP-ENDOR spectra of $\mathrm{ND}_{2} \mathrm{Y}_{731} \bullet$ under similar experimental conditions (Fig. 7.1) reveals the superior performance of WOP CP-ENDOR for investigating small hfcs. In general, three characteristic tensor features arising from hfc interaction with the two amino deuterons (D1, D2) and one perpendicular H-bond (D3) can be assigned. All three features can be clearly identified in the WOP CPENDOR spectra. While WOP CP-ENDOR provides good $\mathrm{S} / \mathrm{N}$ and an almost blind spot free line shape, all other standard ENDOR sequences suffer from either one or the other. In case of Davies ENDOR almost the entire ENDOR spectrum overlaps with the broad central blind spot which results in very low S/R. Mims ENDOR with $\tau=200 \mathrm{~ns}$ results in a reasonable $\mathrm{S} / \mathrm{N}$ but the line shape distortion due to the Mims blind spots cover part of the tensor line shape. Mims ENDOR with $\tau$-average shows the correct hf-tensors but the $\mathrm{S} / \mathrm{N}$ is compromised due to a multitude of Mims blind spots. An alternative approach to overcome this issue is analyzing multiple Mims ENDOR spectra with different $\tau$-values. Considering the idea of identical averaging time, the data set presented in Fig. 7.3 provides the basis for the analysis. It is expected that the $\mathrm{S} / \mathrm{N}$ of the individual spectra is unsatisfying for a robust fitting of parameters. Consequently, this approach requires more acquisition time. Therefore, WOP CP-ENDOR is suggested as the method of choice for the detection of small deuterium hfcs.

The simulation routine is capable of simulating complex orientation-selective 2D CPENDOR spectra which qualitatively reproduce the experiments. However, the 2D $C P$ 
simulation requires extensive calculation time on a well-equipped computer/cluster because the routine diagonalizes the $54 \times 54$ Hamiltonian for a relatively large 2D map of parameters. In conclusion, the WOP CP-ENDOR spectra calculated with the $C P$ simulation approach are in good agreement with the ENDOR spectra obtained from the Tensor simulation. Technical key difference between Tensor simulation and CP simulation is the underlying calculation procedure of the ENDOR transitions. The Tensor simulation predicts ENDOR transitions according to the analytically derived resonance conditions (Eq. 2.34). Thus, a 'stick'-spectrum is generated and processed with line broadening in a straightforward, post-processing step. Likewise, the central blind spot is added in a post-processing step which reduces ENDOR intensity around the nuclear Larmor frequency. Both aspects are intrinsically different in the CP simulation. Here, the ENDOR transitions are obtained from numerically mapping the density matrix evolution under pulse excitation. The central blind spot is intrinsically encoded in the simulation according to the microwave amplitude $\omega_{1 \mathrm{e}} / 2 \pi$ which is in agreement with its physical origin, as introduced in the previous chapter.

Since the spinlock ENDOR and CP simulation routine result in identical tensor line shape (Fig. 7.9), two main conclusions are drawn: 1) The summation of the 2D CPENDOR spectra results in the correct ENDOR spectrum, without introducing line shape artefacts arising from e.g. residual CP effect, unsatisfactory 2D accumulation or similar effects. 2) For ENDOR line shape analysis, the faster spinlock ENDOR simulation routine can be used. Thus, the $C P /$ spinlock simulation routine offers an alternative, non-post-processed tool to analyze ENDOR spectra of complex spin systems.

These conclusions raise the question whether the spinlock simulation approach can be translated into an experimental method in order to directly obtain the WOP spinlock ENDOR spectrum. A possible benefit could be strongly increased S/N. This can concluded from the consideration of a $S=1 / 2, I=1 / 2$ powder-like spin system shown in Fig. 7.11. CP-ENDOR intensities were predicted by RIZZATO et al.27. For simplification, two spin packets with equal hfc, shifted by exactly $A / 2$ due to $g$-anisotropy are considered (Fig. 7.11, A). 


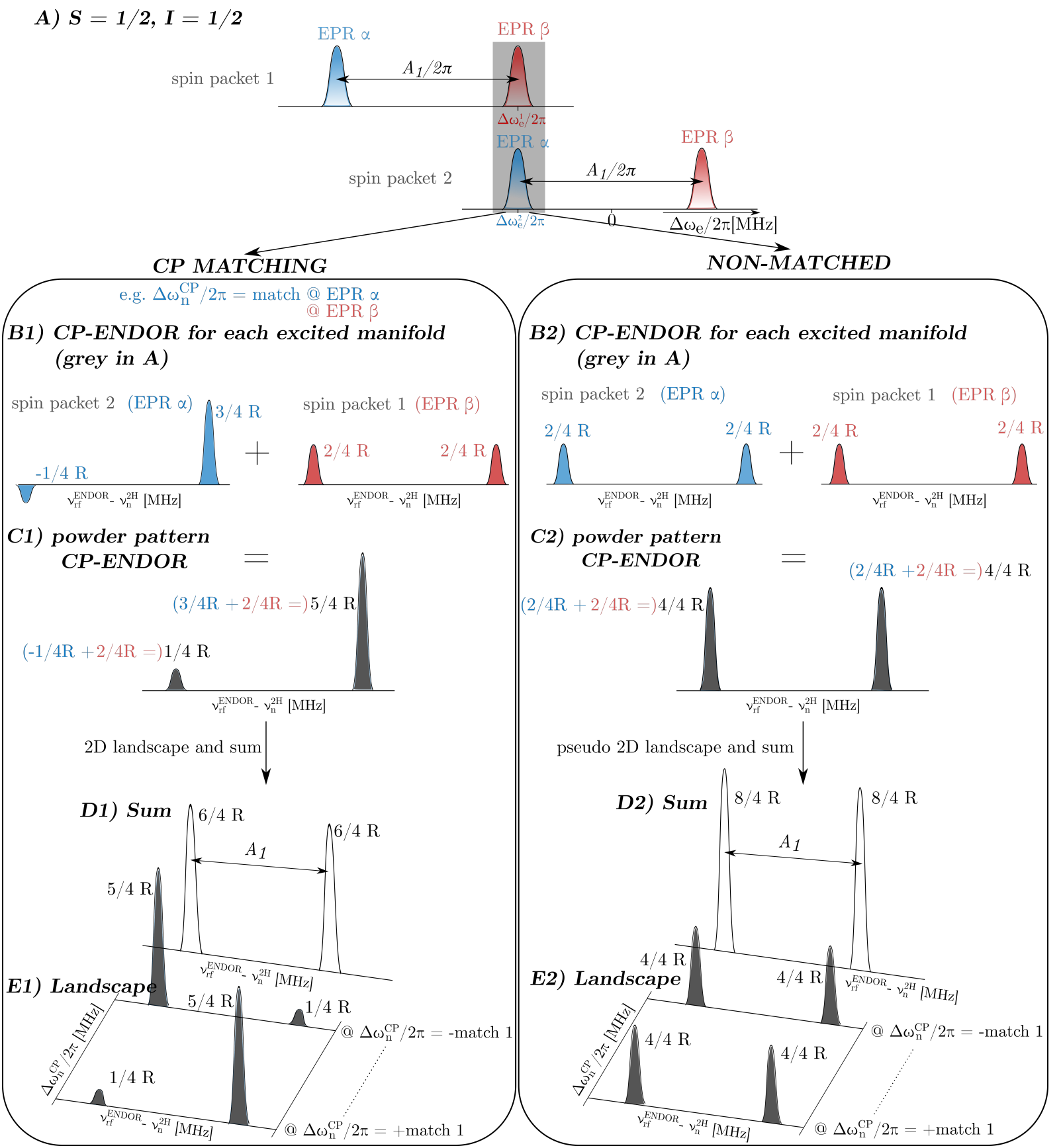

Figure 7.11: Illustration of 2D CP-ENDOR summation and pseudo spinlock ENDOR summation in powder samples. A) In this simplified case two spin packets with equal hfc, shifted by exactly $A / 2 \pi$ due to $g$-anisotropy, are considered $(S=1 / 2, I=1 / 2)$. B1) At the resonance position (grey box) and setting the $\mathrm{CP}$ rf offset to a $\mathrm{CP}$ matching condition of EPR $\alpha$ (spin packet 1 ), this $\mathrm{rf}$ offset cannot satisfy a matching condition of simultaneously excited EPR $\beta$ of spin package 1. C1) The resulting powder pattern intensities are $1 / 4 \mathrm{R}: 5 / 4 \mathrm{R}$. E1) In the 2D landscape the opposite enhancement pattern at the negative rf offset $\left(-\Delta \omega_{\mathrm{n}}^{\mathrm{CP}} / 2 \pi\right)$ is predicted. Summation of the landscape $(D 1)$ results in equal peak intensities of $6 / 4 \mathrm{R}$. The case without $\mathrm{CP}$ matching (no rf pulse) is shown in the right column (B2-E2). Without rf irradiation during $\mathrm{CP}$, both manifolds show equal intensities $(B 2)$. The intensities of each manifold are considered to represent the powder intensities $(C 2)$. From a pseudo 2D landscape (E2), ENDOR intensities of $8 / 4 \mathrm{R}$ are predicted after summation of the landscape $(D 2)$. Intensities are considered as predicted by RizzATo et al.27. 
One EPR transition out of each spin packet is on-resonant (spin package $2 \rightarrow$ EPR $\alpha$, spin package $1 \rightarrow$ EPR $\beta$ ) at the given resonance position (grey box). If, the rf offset $\Delta \omega_{\mathrm{n}}^{\mathrm{CP}} / 2 \pi$ is set to match CP on EPR $\alpha$ of spin package 2 , this offset cannot satisfy the matching condition of the simultaneously excited EPR $\beta$ transition of spin package 1 (B1). Consequently, peak intensities of 1/4 R : 5/4 R (Fig. 7.11, C1) are predicted in this simplified powder example. Further, the opposite enhancement pattern at the negative rf offset $\left(-\Delta \omega_{\mathrm{n}}^{\mathrm{CP}} / 2 \pi\right)$ is obtained for the schematic $2 \mathrm{D}$ landscape $(E 1)$. After summation of the landscape $(D 1)$ equal peak intensities of $6 / 4 \mathrm{R}$ are calculated.

The case without CP matching (no rf pulse during $\mathrm{CP}$ ) is shown in the right column (B2-E2) of Fig. 7.11. Without rf irradiation during CP both manifolds show equal peak intensities of $2 / 4 \mathrm{R}: 2 / 4 \mathrm{R}(B 2)$. The intensities of both manifolds are considered to represent the powder pattern intensities $(C 2)$. Finally, intensities of $8 / 4 \mathrm{R}$ are predicted for each peak of the ENDOR spectrum (D2) after summation of a pseudo 2D landscape $(E 2)$. Thus, the spinlock ENDOR approach would result in $8 / 4 \mathrm{R}-6 / 4 \mathrm{R}=2 / 4 \mathrm{R}$ higher intensity. However, this only holds for sufficiently long sequence repetition delays, and thus, for relaxation of electron and particularly nuclear spins to Boltzmann equilibrium. Otherwise, the predicted $2 / 4 \mathrm{R}$ intensity benefit can be diminished by nuclear saturation under fast accumulation. A recent publication [26] has demonstrated the robustness of the CP-ENDOR sequence towards nuclear-saturation induced signal losses in contrast to to Davies ENDOR. The authors report that CP-ENDOR can be performed with a sequence repetition time on the time scale of the electron spin relaxation process contrary to Davies ENDOR, where the nuclear relaxation rate limits the repetition rate (see also Subsec. 2.3.5 and Subsec. 2.4.2). However, according to the current understanding of the relaxation properties, the spinlock ENDOR sequence should perform similar to Davies ENDOR. Consequently, for sequence repetition rates faster than the nuclear relaxation rate, the additional 2/4 $\mathrm{R}$ gain in intensity becomes jeopardised. Unfortunately, the nuclear relaxation time is difficult to determine from an EPR experiment, but may be up to several orders of magnitude longer than the electron spin lattice relaxation, i.e. for low-gamma ${ }^{2} \mathrm{H}$ nuclei at low $T$.

Another advantage of spinlock ENDOR over 2D CP-ENDOR is that it requires less experimental effort and less $2 \mathrm{D}$ data processing. The straight-forward $1 \mathrm{D}$ experiment eventually provides a user-friendly alternative to the sophisticated 2D WOP CP-ENDOR experiment. This can be done without identifying the matching condition range from a separate eNCP experiment beforehand. In a nutshell, the spinlock ENDOR sequence 
can be applied when relaxation issues are negligible. Thus it is favorable for studies at higher/medium $T$ and high- $\gamma$ nuclei (like protons $/{ }^{1} \mathrm{H}$ ). In other cases, the 2D WOP CP-ENDOR experiment is the better method despite the increased effort.

A suggestion to reduce the workload for performing a 2D WOP CP-ENDOR on complex protein samples is to skip the eNCP experiment. Particularly as the gain in information provided by the eNCP spectrum does not justify the additional averaging time. The eNCP spectrum of the amino tyrosyl radical shown in Fig. $\mathrm{H3}$ of Appendix $\mathrm{H}$ was recorded with almost the same acquisition time as the CP-ENDOR spectra shown in Fig. 7.1. However, the $\mathrm{S} / \mathrm{N}$ is only sufficient to extract broad featureless ranges of electron spin depolarization. The same information can be analytically calculated or simulated considering the minimum/maximum hfc value and the applied $\omega_{1 \mathrm{e}} / 2 \pi$. Thus, it is recommended to skip the eNCP experiment for more challenging samples (low radical yield) and scale the chosen rf offset $\Delta \omega_{\mathrm{n}}^{\mathrm{CP}} / 2 \pi$ according to numerical or analytical predictions.

\subsubsection{WOP CP-ENDOR for $\mathrm{ND}_{2}$-conformation studies}

Orientation-selective WOP CP-ENDOR studies provide evidence for an almost planar configuration $\left(\phi=(0 \pm 20)^{\circ}\right)$ of the amino group and have confirmed the DFT predictions $\frac{17}{17}$. This is consistent with the determination on the basis of multifrequency EPR and $34 \mathrm{GHz}$ ENDOR studies using the $\mathrm{D}_{6}-\mathrm{NH}_{2} \mathrm{Y}_{731}-\alpha 2$ labeling approach 86 . The absence of blind spots in contrast to Mims ENDOR spectra is a key benefit of the WOP CP-ENDOR sequence. It allows for interpretation and analysis of the amino deuteron hfc-tensors. In this context, the good agreement between the two simulation approaches (spinlock and Tensor sim) is highlighted (see Figs. 7.6, 7.10).

The characterization is not only important in terms of spectroscopic validation of DFT parameters, but has resulted in a re-interpretation of the redox potential landscape of $\mathrm{NH}_{2} \mathrm{Ys}$ within the radical transfer pathway and activity of the mutant 86 . The amino mutation is known to lower the redox potential at the specific $\mathrm{Y}$ by several hundreds of $\mathrm{mV}$ (e.g. $587 \mathrm{mV}$ for $\mathrm{NH}_{2} \mathrm{Y}_{731}-\alpha 2$ ) and is the origin of the well-established spin trap functionality ${ }^{[79]}$ (see also Fig. 2.16 in Sec.2.5). Further, the reported mutant activity with CDP/ATP of 3-5\% (relative to the wt-RNR) was rationalized by a protein-induced geometry distortion in order to bypass the large redox potential gap to the next the pathway 106$]$ residue. Hence, distortion was assumed to tune the redox potential of the $\mathrm{NH}_{2} \mathrm{Y}$ ground state and or $\mathrm{NH}_{2} \mathrm{Y}$ radical state in order to maintain protein activity 106 . 
According to the results obtained with WOP CP-ENDOR in this work, in agreement with the most recent multifrequency EPR study, the $\mathrm{NH}_{2} \mathrm{Y}$ radical conformation is planar. Thus, it preserves the high energy barrier to the following Y residue. The reported 3-5\% mutant activity has been associated with wt contamination introduced by discrimination difficulties between $\mathrm{NH}_{2} \mathrm{Y}$ and $\mathrm{Y}$ of the tRNA/PS pair 86 .

Finally, planar configurations of the neighboring $\mathrm{NH}_{2} \mathrm{Y}_{730^{-}} \alpha 2$ and $\mathrm{NH}_{2} \mathrm{Y}_{356^{-}} \beta 2$ are also predicted on the basis of DFT calculations $[6]$, while spectroscopic evidence lacks because incorporation of $\mathrm{D}_{6}-\mathrm{NH}_{2} \mathrm{Y}$ at the respective $\mathrm{Y}$ 's was not yet successfully. Based on the results in this work, evidence for the planarity of $\mathrm{NH}_{2} \mathrm{Y}_{730^{-}} \alpha 2$ and $\mathrm{NH}_{2} \mathrm{Y}_{356^{-}} \beta 2$ can be provided straight-forward from ${ }^{2} \mathrm{H}$ WOP CP-ENDOR investigations on the respective $\mathrm{ND}_{2} \mathrm{Ys}$, without the need for the alternative, synthetically challenging mutants. 



\section{Conclusions and perspectives for CP-ENDOR spectroscopy}

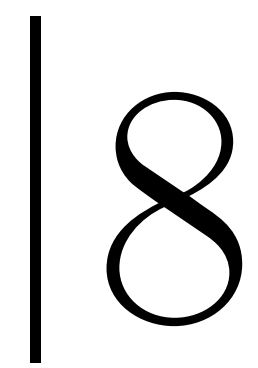

The results presented in this thesis illustrate the capability of $94 \mathrm{GHz} / 3.4 \mathrm{~T}^{2} \mathrm{H} \mathrm{CP}-$ ENDOR to detect small hyperfine couplings between electron and nuclear spins with high sensitivity. The proposed WOP CP-ENDOR sequence was demonstrated to be superior compared to the well-established Davies and Mims ENDOR as well as to the standard CP-ENDOR sequence. At first, the fundamental physical framework for performing cross-polarization between an electron spin and deuterium nuclei was established by experiments on a single crystal of malonic acid radicals in conjunction with theoretical predictions. Secondly, a modification of the standard CP-ENDOR sequence (namely WOP CP-ENDOR) with enhanced sensitivity for the detection of small hyperfine couplings was investigated through experiments and simulations on the organic ${ }^{2} \mathrm{H}-\mathrm{BDPA}$ radical. Finally, the WOP CP-ENDOR sequence was applied to the radical intermediate $\mathrm{ND}_{2} \mathrm{Y}_{731}{ }^{\bullet}$ in $E$. coli $\mathrm{RNR}$ for the examination of the structure of the amino group.

In Chapter 5 the matching conditions were determined from eNCP experiments on the deuterated malonic acid radical in a $\gamma$-irradiated single crystal. The experimentally observed matching conditions were validated by analytical and numerical calculations. The latter were obtained from a simulation routine developed on the basis of density matrix formalism. The subsequent CP-ENDOR spectra of the deuterated malonic acid radical showed characteristic asymmetric CP-ENDOR spectra as a result of successful electron-nuclear cross polarization transfer.

The detection of small hyperfine couplings in CP-ENDOR was significantly improved when omitting the initial $\pi / 2$ pulse of the CP-ENDOR sequence. The modification was called without preparation pulse (WOP) CP-ENDOR (Chapter 6). Comparative experiments were performed on the organic radical ${ }^{2} \mathrm{H}-\mathrm{BDPA}$ and the CP-ENDOR 
spectra reproduced by a numerical simulation routine. WOP CP-ENDOR led to a gain in signal-to-noise ratio by a factor of five, corresponding to 25 times less averaging time regarding the detection of hfcs $\leqslant 1 \mathrm{MHz}$. This enhancement was assigned to different excitation profiles of the CP-ENDOR sequences performed with or without the initial $\pi / 2$ pulse. Those excitation profiles are the origin of a central blind spot of varying width in the CP-ENDOR spectrum. The excitation profile of the WOP CP-ENDOR sequence was determined to be narrower compared to the standard CP-ENDOR sequence from numerical simulations. Derived from the analytically and numerically calculated excitation profiles, the width of the central blind spot $\Delta \omega_{1 / 2}$ in WOP CP-ENDOR depends on the microwave field strength $\omega_{1 e} / 2 \pi$. Therefore, this parameter becomes crucial for the optimal detection of small hyperfine couplings in CP-ENDOR experiments.

The numerical simulation routine developed in the framework of this thesis not only facilitates the calculation of excitation profiles, but provides a robust access to matching conditions and CP-ENDOR intensities. Remarkably, powder-pattern 2D CP-ENDOR spectra of a spin system with multiple coupled deuterium nuclei $\left({ }^{2} \mathrm{H}-\mathrm{BDPA}\right.$ radical $\left./ \mathrm{ND}_{2} \mathrm{Y}_{731}{ }^{\bullet}\right)$ were simulated. The powder-pattern ${ }^{2} \mathrm{H}$-BDPA experiments are in good agreement with the numerical simulations and reproduce the line shape as well as the difference in signal intensity between the CP-ENDOR sequence variations. This allows for future analysis and interpretation of 2D CP-ENDOR spectra.

Finally, the investigations on the radical intermediate $\mathrm{ND}_{2} \mathrm{Y}_{731} \bullet$ in E. coli RNR presented in Chapter 7 highlight the advantages of WOP CP-ENDOR in terms of S/N and line shape in comparison to the well-established ENDOR sequences. In the EPR literature, the method of choice for the detection of small deuterium hfes was and still is Mims ENDOR where the Mims blind spots are accepted as inevitable. The WOP CP-ENDOR sequence, however, has unmasked hfc-tensor features of the amino group which were distorted by Mims blind spots in the Mims ENDOR spectrum. The advantages of the WOP CP-ENDOR sequence allowed the establishment of the planarity of the functional group.

The promising results of the tyrosyl radical intermediate in E. coli RNR encourage future applications of WOP CP-ENDOR on radical intermediates such as $\mathrm{ND}_{2} \mathrm{Y}_{730}$ and $\mathrm{ND}_{2} \mathrm{Y}_{356} \bullet$ to study the $\mathrm{ND}_{2}$ structure of the respective amino tyrosyl radical. Indeed, the WOP CP-ENDOR sequence is neither restricted to these unnatural amino acids nor to the E. coli RNR enzyme, not even to the deuterium nuclei. Its enhanced performance can be beneficial for studies on other biological systems and/or further nuclei of interest 
in ENDOR spectroscopy such as ${ }^{14} \mathrm{~N},{ }^{13} \mathrm{C}$ or ${ }^{17} \mathrm{O}$.

For the study of spin systems for which nuclear saturation issues can be excluded, the performance of the proposed spinlock WOP ENDOR sequence should be evaluated. This CP-transfer free 1D ENDOR alternative, derived from the WOP CP-ENDOR sequence, is predicted to result in even higher sensitivity when compared to the $2 \mathrm{D}$ WOP CP-ENDOR spectrum. The improvement was calculated on the basis of S/N losses upon summation of the 2D CP-ENDOR spectra. Further, the spinlock WOP ENDOR sequence would be easier to optimize since the CP-offset parameter is not necessary. The sequence offers the best performance when nuclear relaxation issues are negligible, like for instance for studies at higher/medium $T$ or on high- $\gamma$ nuclei.

A general pathway which may provide further CP-ENDOR signal gain is the implementation of so-called optimal control pulses. Such pulses can be generated by an arbitrary wave form generator operating at high microwave frequencies. These have become commercially available during the last decade. Recently, composite 107 or shaped pulses ${ }^{[108,109]}$ were in the focus of cutting-edge developments for optimal electron polarization transfer. The CP-ENDOR experiment may, therefore, be further improved using a CP-pulse designed for optimal electron-nuclear cross polarization transfer. The simulation routine developed in this thesis provides a basis for the required optimization algorithm. 



\section{Appendix}

\section{A. Davies ENDOR on a single crystal of a malonic acid radical}

Davies ENDOR was perfomed selectively on each EPR transition $(\alpha, \beta$ and $\gamma)$ of the single crystal of malonic acid mounted in the same orientation as for the CP-ENDOR experiments.

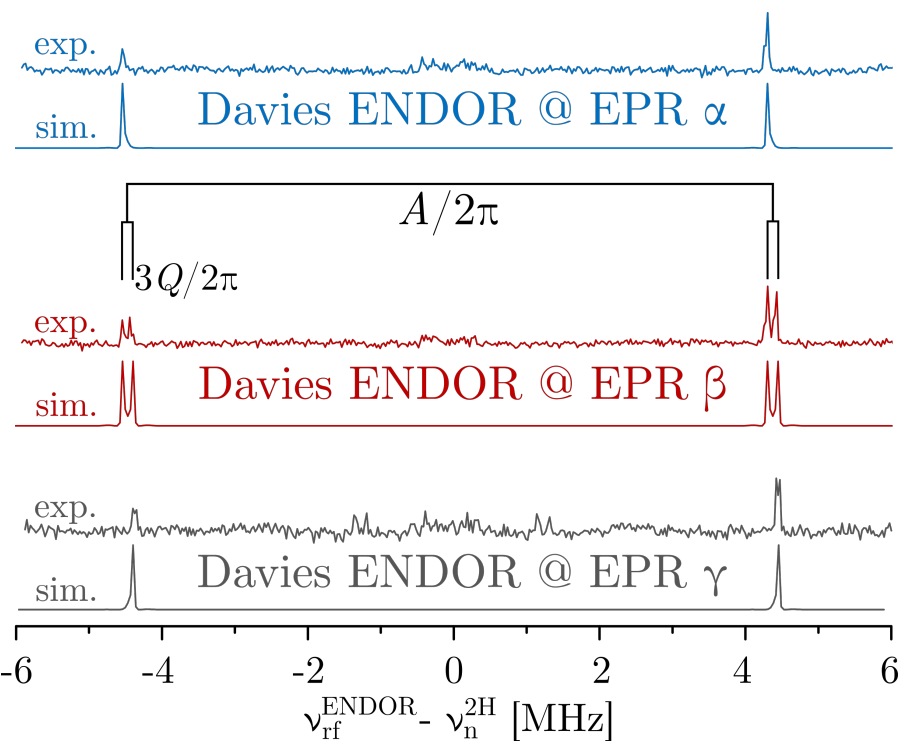

Figure A1: Selective Davies ENDOR of a single crystal of deuterated malonic acid. Exp. conditions: $T=40 \mathrm{~K}, \nu_{\mathrm{EPR}}=94 \mathrm{GHz}$, shot repetition time $=$ $100 \mathrm{~ms} ; t_{\pi / 2, \mathrm{mw}}=400 \mathrm{~ns}, t_{\pi, \mathrm{rf}}=28 \mu \mathrm{s}, \omega_{1 \mathrm{n}} / 2 \pi=16 \mathrm{kHz}, 1 \mathrm{shot} /$ point, random rf acquisition, 20 scans each. Sim. parameters: $A / 2 \pi=-8.84 \mathrm{MHz}, Q / 2 \pi=-0.04 \mathrm{MHz}$, $t_{\pi, \mathrm{rf}}=28 \mu \mathrm{s}, \omega_{1 \mathrm{n}} / 2 \pi=7 \mathrm{kHz}, \Delta \omega_{\mathrm{e}}= \pm A / 2 \pi, 0$.

The determined parameters are $A / 2 \pi=-8.84 \mathrm{MHz}$ and $Q / 2 \pi=-0.04 \mathrm{MHz}$. The negative signs were adapted from Literature 28 . The simulation was performed with a simulation routine developed in this thesis on basis of the formalism of the CP-ENDOR 
simulation. The experimental as well as simulation parameters are summarized in the figure caption. Asymmetry of the ENDOR lines could be reproduced in a simulation

of the Davies-ENDOR spectra using the 'salt'-routine of the software Easyspin [96] and considering hf-enhancement (data not shown). This effect could not be reproduced by our numerical simulations, which assume high-field conditions.

\section{B. Spin operator definitions}

\section{B.1. Electron spin coupled to one nuclear spin-1}

We consider a quantum system that consists of one electron spin $S=1 / 2$ and one nuclear spin $I=1$. The corresponding density-matrix description relies on the generalized Pauli matrices

$$
\begin{aligned}
& \sigma_{x}^{S}:=\frac{1}{2}\left[\begin{array}{ll}
0 & 1 \\
1 & 0
\end{array}\right], \sigma_{y}^{S}:=\frac{1}{2}\left[\begin{array}{cc}
0 & -i \\
i & 0
\end{array}\right], \sigma_{z}^{S}:=\frac{1}{2}\left[\begin{array}{cc}
1 & 0 \\
0 & -1
\end{array}\right], \mathbb{1}^{S}:=\left[\begin{array}{ll}
1 & 0 \\
0 & 1
\end{array}\right] \text { and } \\
& \sigma_{x}^{I}:=\frac{1}{\sqrt{2}}\left[\begin{array}{lll}
0 & 1 & 0 \\
1 & 0 & 1 \\
0 & 1 & 0
\end{array}\right], \sigma_{y}^{I}:=\frac{1}{\sqrt{2}}\left[\begin{array}{ccc}
0 & -1 & 0 \\
1 & 0 & -1 \\
0 & 1 & 0
\end{array}\right], \sigma_{z}^{I}:=\left[\begin{array}{ccc}
1 & 0 & 0 \\
0 & 0 & 0 \\
0 & 0 & -1
\end{array}\right], \mathbb{1}^{I}:=\left[\begin{array}{lll}
1 & 0 & 0 \\
0 & 1 & 0 \\
0 & 0 & 1
\end{array}\right]
\end{aligned}
$$

which are used to define the spin operators

$$
\begin{aligned}
& S_{x}:=\sigma_{x}^{S} \otimes \mathbb{1}^{I}=\frac{1}{2}\left[\begin{array}{cccccc}
0 & 0 & 0 & 1 & 0 & 0 \\
0 & 0 & 0 & 0 & 0 \\
0 & 0 & 0 & 0 & 0 & 1 \\
1 & 0 & 0 & 0 & 0 & 0 \\
0 & 1 & 0 & 0 & 0 & 0 \\
0 & 0 & 1 & 0 & 0 & 0
\end{array}\right], S_{y}:=\sigma_{y}^{S} \otimes \mathbb{1}^{I}=\frac{1}{2}\left[\begin{array}{cccccc}
0 & 0 & 0 & -i & 0 & 0 \\
0 & 0 & 0 & 0 & -i & 0 \\
0 & 0 & 0 & 0 & 0 & 0 \\
i & 0 & 0 & 0 & -i \\
0 & 0 & 0 & 0 & 0 & 0 \\
0 & 0 & i & 0 & 0 & 0 \\
1 & 0 & 0 & 0 & 0
\end{array}\right],
\end{aligned}
$$

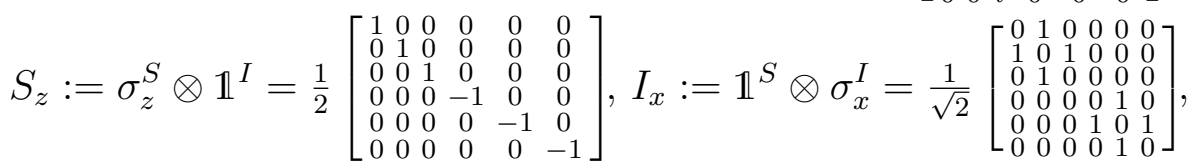

$$
\begin{aligned}
& I_{y}:=\mathbb{1}^{S} \otimes \sigma_{y}^{I}=\frac{1}{\sqrt{2}}\left[\begin{array}{cccccc}
0 & -1 & 0 & 0 & 0 & 0 \\
1 & 0 & -1 & 0 & 0 & 0 \\
0 & 1 & 0 & 0 & 0 & 0 \\
0 & 0 & 0 & 0 & -1 & 0 \\
0 & 0 & 0 & 1 & 0 & -1 \\
0 & 0 & 0 & 0 & 1 & 0
\end{array}\right], I_{z}:=\mathbb{1}^{S} \otimes \sigma_{z}^{I}=\left[\begin{array}{cccccc}
1 & 0 & 0 & 0 & 0 & 0 \\
0 & 0 & 0 & 0 & 0 & 0 \\
0 & 0 & -1 & 0 & 0 & 0 \\
0 & 0 & 0 & 1 & 0 & 0 \\
0 & 0 & 0 & 0 & 0 & 0 \\
0 & 0 & 0 & 0 & 0 & 0 \\
0 & 0 & 0
\end{array}\right] .
\end{aligned}
$$

We also introduce the $6 \times 6$ identity matrix $\mathbb{1}:=\mathbb{1}^{S} \otimes \mathbb{1}^{I}$. 


\section{B.2. Electron spin coupled to two nuclear spins-1}

The generalized Pauli matrices defined in the previous section are used to obtain the spin operators for an electron spin- $1 / 2$ coupled to two nuclear spins- 1 according to

$$
\begin{aligned}
S_{x} & :=\sigma_{x}^{S} \otimes \mathbb{1}^{I, 1} \otimes \mathbb{1}^{I, 2}, \\
S_{y} & :=\sigma_{y}^{S} \otimes \mathbb{1}^{I, 1} \otimes \mathbb{1}^{I, 2}, \\
S_{z} & :=\sigma_{z}^{S} \otimes \mathbb{1}^{I, 1} \otimes \mathbb{1}^{I, 2}, \\
I_{x, 1} & :=\mathbb{1}^{S} \otimes \sigma_{x}^{I, 1} \otimes \mathbb{1}^{I, 2}, \\
I_{y, 1} & :=\mathbb{1}^{S} \otimes \sigma_{y}^{I, 1} \otimes \mathbb{1}^{I, 2}, \\
I_{z, 1} & :=\mathbb{1}^{S} \otimes \sigma_{z}^{I, 1} \otimes \mathbb{1}^{I, 2}, \\
I_{x, 2} & :=\mathbb{1}^{S} \otimes \mathbb{1}^{I, 1} \otimes \sigma_{x}^{I, 2}, \\
I_{y, 2} & :=\mathbb{1}^{S} \otimes \mathbb{1}^{I, 1} \otimes \sigma_{y}^{I, 2}, \\
I_{z, 2} & :=\mathbb{1}^{S} \otimes \mathbb{1}^{I, 1} \otimes \sigma_{z}^{I, 2} .
\end{aligned}
$$

Further, the $18 \times 18$ identity matrix $\mathbb{1}:=\mathbb{1}^{S} \otimes \mathbb{1}^{I, 1} \otimes \mathbb{1}^{I, 2}$ is defined. The Pauli matrices of nucleus two are equal to those of nucleus one.

\section{B.3. Electron spin coupled to three nuclear spins-1}

The generalized Pauli matrices defined in the first section are used to obtain the spin operators for an electron spin- $1 / 2$ coupled to three nuclear spins- 1 according to

$$
\begin{aligned}
S_{x} & :=\sigma_{x}^{S} \otimes \mathbb{1}^{I, 1} \otimes \mathbb{1}^{I, 2} \otimes \mathbb{1}^{I, 3}, \\
S_{y} & :=\sigma_{y}^{S} \otimes \mathbb{1}^{I, 1} \otimes \mathbb{1}^{I, 2} \otimes \mathbb{1}^{I, 3}, \\
S_{z} & :=\sigma_{z}^{S} \otimes \mathbb{1}^{I, 1} \otimes \mathbb{1}^{I, 2} \otimes \mathbb{1}^{I, 3}, \\
I_{x, 1} & :=\mathbb{1}^{S} \otimes \sigma_{x}^{I, 1} \otimes \mathbb{1}^{I, 2} \otimes \mathbb{1}^{I, 3}, \\
I_{y, 1} & :=\mathbb{1}^{S} \otimes \sigma_{y}^{I, 1} \otimes \mathbb{1}^{I, 2} \otimes \mathbb{1}^{I, 3}, \\
I_{z, 1} & :=\mathbb{1}^{S} \otimes \sigma_{z}^{I, 1} \otimes \mathbb{1}^{I, 2} \otimes \mathbb{1}^{I, 3},
\end{aligned}
$$




$$
\begin{aligned}
I_{x, 2} & :=\mathbb{1}^{S} \otimes \mathbb{1}^{I, 1} \otimes \sigma_{x}^{I, 2}, \otimes \mathbb{1}^{I, 3}, \\
I_{y, 2} & :=\mathbb{1}^{S} \otimes \mathbb{1}^{I, 1} \otimes \sigma_{y}^{I, 2}, \otimes \mathbb{1}^{I, 3}, \\
I_{z, 2} & :=\mathbb{1}^{S} \otimes \mathbb{1}^{I, 1} \otimes \sigma_{z}^{I, 2} \otimes \mathbb{1}^{I, 3}, \\
I_{x, 3} & :=\mathbb{1}^{S} \otimes \mathbb{1}^{I, 1} \otimes \mathbb{1}^{I, 2}, \otimes \sigma_{x}^{I, 3}, \\
I_{y, 3} & :=\mathbb{1}^{S} \otimes \mathbb{1}^{I, 1} \otimes \mathbb{1}^{I, 2}, \otimes \sigma_{y}^{I, 3}, \\
I_{z, 3} & :=\mathbb{1}^{S} \otimes \mathbb{1}^{I, 1} \otimes \mathbb{1}^{I, 2} \otimes \sigma_{z}^{I, 3} .
\end{aligned}
$$

Further, the $54 \times 54$ identity matrix $\mathbb{1}:=\mathbb{1}^{S} \otimes \mathbb{1}^{I, 1} \otimes \mathbb{1}^{I, 2} \otimes \mathbb{1}^{I, 3}$ is defined. The Pauli matrices of nucleus three are equal to those of nucleus two and one.

\section{Transformation of spin operators into a new basis}

A second basis for spin operators is introduced which is adapted for the analytical treatment. It simplifies the assignment of energy levels and their corresponding populations in the density matrix throughout the analytical treatment. In this basis the energy levels are numbered according to the energy scheme of Fig. 5.2, A. The elements of the second basis are obtained via the transformation $\tilde{M}=P M P$ where

$$
P=\left[\begin{array}{ll}
1 & 0 \\
0 & 1
\end{array}\right] \otimes\left[\begin{array}{lll}
0 & 0 & 1 \\
0 & 1 & 0 \\
1 & 0 & 0
\end{array}\right]=\left[\begin{array}{llllll}
0 & 0 & 1 & 0 & 0 & 0 \\
0 & 1 & 0 & 0 & 0 & 0 \\
1 & 0 & 0 & 0 & 0 & 0 \\
0 & 0 & 0 & 0 & 0 & 1 \\
0 & 0 & 0 & 0 & 1 & 0 \\
0 & 0 & 0 & 1 & 0 & 0
\end{array}\right]=P^{-1}
$$

This maps the vector basis

$$
M=\left[\begin{array}{l}
\alpha \alpha \\
\alpha \beta \\
\alpha \gamma \\
\beta \alpha \\
\beta \beta \\
\beta \gamma
\end{array}\right] \stackrel{P M P}{\longrightarrow}\left[\begin{array}{l}
\alpha \gamma \\
\alpha \beta \\
\beta \gamma \\
\beta \beta \\
\beta \alpha
\end{array}\right]
$$


and the general matrix

$$
\left[\begin{array}{cccccc}
M_{11} & M_{12} & M_{13} & M_{14} & M_{15} & M_{16} \\
M_{21} & M_{22} & M_{23} & M_{24} & M_{25} & M_{26} \\
M_{31} & M_{32} & M_{33} & M_{34} & M_{35} & M_{36} \\
M_{41} & M_{42} & M_{43} & M_{44} & M_{45} & M_{46} \\
M_{51} & M_{52} & M_{53} & M_{54} & M_{55} & M_{56} \\
M_{61} & M_{62} & M_{63} & M_{64} & M_{65} & M_{66}
\end{array}\right]
$$

is mapped to

$$
\left[\begin{array}{llllll}
M_{33} & M_{32} & M_{31} & M_{36} & M_{35} & M_{34} \\
M_{23} & M_{22} & M_{21} & M_{26} & M_{25} & M_{24} \\
M_{13} & M_{12} & M_{11} & M_{16} & M_{15} & M_{14} \\
M_{63} & M_{62} & M_{61} & M_{66} & M_{65} & M_{64} \\
M_{53} & M_{52} & M_{51} & M_{56} & M_{55} & M_{54} \\
M_{43} & M_{42} & M_{41} & M_{46} & M_{45} & M_{44}
\end{array}\right] .
$$

In particular, we obtain

$$
\begin{gathered}
\left.\tilde{S}_{z}=S_{z}=1 / 2\left[\left[\begin{array}{c}
1 \\
1 \\
1 \\
-1 \\
-1 \\
-1
\end{array}\right]\right], \tilde{I}_{z}=-I_{z}=\left[\begin{array}{c}
-1 \\
0 \\
1 \\
-1 \\
0 \\
1
\end{array}\right]\right], \tilde{S}_{x}=S_{x}=1 / 2\left[\begin{array}{cccccc}
0 & 0 & 0 & 1 & 0 & 0 \\
0 & 0 & 0 & 0 & 1 & 0 \\
0 & 0 & 0 & 0 & 0 & 1 \\
1 & 0 & 0 & 0 & 0 & 0 \\
0 & 1 & 0 & 0 & 0 & 0 \\
0 & 0 & 1 & 0 & 0 & 0
\end{array}\right], \\
\tilde{I}_{x}=I_{x}=1 / \sqrt{2}\left[\begin{array}{cccccc}
0 & 1 & 0 & 0 & 0 & 0 \\
1 & 0 & 1 & 0 & 0 & 0 \\
0 & 1 & 0 & 0 & 0 & 0 \\
0 & 0 & 0 & 0 & 1 & 0 \\
0 & 0 & 0 & 1 & 0 & 1 \\
0 & 0 & 0 & 0 & 1 & 0
\end{array}\right], \tilde{I}_{y}=-I_{y}, \tilde{S}_{y}=S_{y} .
\end{gathered}
$$

Here, double-square brackets [[]] denote a diagonal matrix with the given diagonal entries. 


\section{Analytically calculated CP-ENDOR intensities for EPR $\gamma$ and EPR $\beta$ of the malonic acid radical}

In the following sections, the CP-ENDOR intensities for selective excitation of EPR $\gamma$ and $\beta$ of the malonic acid single crystal are calculated. The treatment is analogous to EPR $\alpha$ in Subsec. 5.3 .2 and considers the matching conditions in Table. 5.3 .

\section{D.1. Analytically calculated CP-ENDOR intensities for EPR $\gamma$ excitation}

When EPR excitation occurs selectively on the EPR $\gamma$ transition ([1-4]) by means of

a $\left(\frac{\pi}{2}\right)_{x}$ mw pulse the initial density matrix $\rho_{0}$ in the doubly rotating frame becomes $\rho\left(0^{+}\right)$:

$$
\begin{aligned}
\rho\left(0^{+}\right) & =e^{-i \frac{\pi}{2} I_{x}^{1-4}}\left\{-R\left(I_{z}^{1-4}+I_{z}^{2-5}+I_{z}^{3-6}\right)\right\} e^{i \frac{\pi}{2} I_{x}^{1-4}} \\
& =-R\left(-I_{y}^{1-4}+I_{z}^{2-5}+I_{z}^{3-6}\right)
\end{aligned}
$$

In the next step, we transfer $\rho\left(0^{+}\right)$into the tilted frame of the $\gamma$ manifold using the transformation as given in Eq. 5.23 and the rotation angles for the malonic acid case $\theta_{\alpha}=\pi, \theta_{\beta}=\pi, \theta_{\gamma}=\pi / 2$ according to the definitions in Table 5.2. Transformation into the tilted frame and letting the off-diagonal elements decay results in

$$
\begin{aligned}
\rho^{\prime}\left(0^{+}\right) & =U_{x}\left(\theta_{\gamma}, \theta_{\beta}, \theta_{\alpha}\right)\left\{\rho\left(0^{+}\right)\right\} U_{x}^{-1}\left(\theta_{\gamma}, \theta_{\beta}, \theta_{\alpha}\right) \\
& =U_{x}(\pi / 2, \pi, \pi)\left\{-R\left(-I_{y}^{1-4}+I_{z}^{2-5}+I_{z}^{3-6}\right)\right\} U_{x}^{-1}(\pi / 2, \pi, \pi) \\
& =+R I_{z}^{1-4}+R I_{z}^{2-5}+I_{z}^{3-6} \\
& {\left.\left[\begin{array}{c}
R / 2 \\
R / 2 \\
R / 2 \\
-R / 2 \\
-R / 2 \\
-R / 2
\end{array}\right]\right] }
\end{aligned}
$$


If at this stage rf irradiation creates degeneracy between levels $\left[1^{\prime}-5^{\prime}\right]$ or $\left[2^{\prime}-4^{\prime}\right]$ we obtain

$$
\begin{aligned}
\rho^{\prime}\left(t_{\text {spinlock }}\right)= & {\left[\left[\begin{array}{c}
R / 2 \\
R / 2 \\
R / 2 \\
-R / 2 \\
-R / 2 \\
-R / 2
\end{array}\right]\right] \rightarrow \rho^{\prime}\left(t_{\mathrm{CP}}^{\left[1^{\prime}-5^{\prime}\right]}\right)=\left[\left[\begin{array}{c}
0 \\
R / 2 \\
R / 2 \\
-R / 2 \\
0 \\
\left.\rho^{\prime}\left(t_{\text {spinlock }}\right)=\left[\left[\begin{array}{c}
R / 2 \\
R / 2 \\
R / 2 \\
-R / 2 \\
-R / 2 \\
-R / 2
\end{array}\right]\right] \rightarrow \rho^{\prime}\left(t_{\mathrm{CP}}^{\left[2^{\prime}-4^{\prime}\right]}\right)=\left[\begin{array}{c} 
\\
-R / 2
\end{array}\right]\right] \\
R / 2 \\
0 \\
R / 2 \\
0 \\
-R / 2 \\
-R / 2
\end{array}\right]\right] }
\end{aligned}
$$

Rotation back in the doubly rotating frame is performed via

$$
\begin{aligned}
\rho\left(t_{\mathrm{CP}}^{\left[i^{\prime}-j^{\prime}\right]}\right) & =U_{x}^{-1}\left(\theta_{\gamma}, \theta_{\beta}, \theta_{\alpha}\right)\left\{\rho^{\prime}\left(t_{\mathrm{CP}}^{\left[i^{\prime}-j^{\prime}\right]}\right)\right\} U_{x}\left(\theta_{\gamma}, \theta_{\beta}, \theta_{\alpha}\right) \\
& =U_{x}^{-1}(\pi / 2, \pi, \pi)\left\{\rho^{\prime}\left(t_{\mathrm{CP}}^{\left[i^{\prime}-j^{\prime}\right]}\right)\right\} U_{x}(\pi / 2, \pi, \pi)
\end{aligned}
$$

from which we obtain back in the doubly rotating frame

$$
\left.\rho\left(t_{\mathrm{CP}}^{\left[1^{\prime}-5^{\prime}\right]}\right)=\left[\left[\begin{array}{c}
R / 4 \\
-R / 2 \\
-R / 2 \\
-R / 4 \\
0 \\
R / 2
\end{array}\right]\right], \rho\left(t_{\mathrm{CP}}^{\left[2^{\prime}-4^{\prime}\right]}\right)=\left[\begin{array}{c}
-R / 4 \\
0 \\
-R / 2 \\
-R / 4 \\
R / 2 \\
R / 2
\end{array}\right]\right]
$$

From these matrices we can for example calculate the CP-ENDOR signal intensities according to

$$
\begin{aligned}
S_{\text {echo, } \gamma}^{\mathrm{NMR} 1-2}\left(\mathrm{CP}\left[1^{\prime}-5^{\prime}\right]\right) & \sim \operatorname{Tr}\left\{\left(U_{\pi}^{1-2}\right) \rho\left(t_{\mathrm{CP}}^{\left[1^{\prime}-5^{\prime}\right]}\right)\left(U_{\pi}^{1-2}\right)^{-1} I_{z}^{1-4}\right\} \\
& \sim \operatorname{Tr}\left\{\rho\left(t_{\mathrm{CP}}^{\left[1^{\prime}-5^{\prime}\right]}\right)\left(U_{\pi}^{1-2}\right)^{-1} I_{z}^{1-4}\left(U_{\pi}^{1-2}\right)\right\} \\
& \sim \operatorname{Tr}\left\{\rho\left(t_{\mathrm{CP}}^{\left[1^{\prime}-5^{\prime}\right]}\right) I_{z}^{2-4}\right\}
\end{aligned}
$$


and

$$
S_{\text {echo, } \gamma}^{\mathrm{NMR}}{ }^{-5}\left(\mathrm{CP}\left[1^{\prime}-5^{\prime}\right]\right) \sim \operatorname{Tr}\left\{\rho\left(t_{\mathrm{CP}}^{\left[1^{\prime}-5^{\prime}\right]}\right) I_{z}^{1-5}\right\}
$$

The calculated CP-ENDOR intensities are listed in Table 5.4.

\section{D.2. Analytically calculated CP-ENDOR intensities for EPR $\beta$ excitation}

When EPR excitation occurs selectively on the EPR $\beta$ transition ([2-5]) by means of

a $\left(\frac{\pi}{2}\right)_{x}$ mw pulse the initial density matrix $\rho_{0}$ in the doubly rotating frame becomes $\rho\left(0^{+}\right)$:

$$
\begin{aligned}
\rho\left(0^{+}\right) & =e^{-i \frac{\pi}{2} I_{x}^{2-5}}\left\{-R\left(I_{z}^{1-4}+I_{z}^{2-5}+I_{z}^{3-6}\right)\right\} e^{i \frac{\pi}{2} I_{x}^{2-5}} \\
& =-R\left(I_{z}^{1-4}-I_{y}^{2-5}+I_{z}^{3-6}\right)
\end{aligned}
$$

In the next step, we transfer $\rho\left(0^{+}\right)$into the tilted frame of the $\beta$ manifold using the matrix $U_{x}\left(\theta_{\gamma}, \theta_{\beta}, \theta_{\alpha}\right)$ as given in Eq. 5.2.2 and the rotation angles for the malonic acid case $\theta_{\alpha}=\pi, \theta_{\beta}=\pi / 2, \theta_{\gamma}=0$ according to the definitions in Table 5.2. Transformation into the tilted frame and letting the off-diagonal elements decay results in

$$
\begin{aligned}
\rho^{\prime}\left(0^{+}\right) & =U_{x}\left(\theta_{\gamma}, \theta_{\beta}, \theta_{\alpha}\right)\left\{\rho\left(0^{+}\right)\right\} U_{x}^{-1}\left(\theta_{\gamma}, \theta_{\beta}, \theta_{\alpha}\right) \\
& =U_{x}(0, \pi / 2, \pi)\left\{-R\left(I_{z}^{1-4}-I_{y}^{2-5}+I_{z}^{3-6}\right]\right\} U_{x}^{-1}(0, \pi / 2, \pi) \\
& =-R I_{z}^{1-4}+R I_{z}^{2-5}+R I_{z}^{3-6} \\
& {\left[\left[\begin{array}{c}
-R / 2 \\
R / 2 \\
R / 2 \\
R / 2 \\
-R / 2 \\
-R / 2
\end{array}\right]\right] }
\end{aligned}
$$


If at this stage rf irradiation creates degeneracy between levels $\left[1^{\prime}-2^{\prime}\right],\left[2^{\prime}-6^{\prime}\right],\left[3^{\prime}-5^{\prime}\right]$ or $\left[4^{\prime}-5^{\prime}\right]$ we obtain

$$
\begin{aligned}
& \rho^{\prime}\left(t_{\text {spinlock }}\right)=\left[\left[\begin{array}{c}
-R / 2 \\
R / 2 \\
R / 2 \\
R / 2 \\
-R / 2 \\
-R / 2
\end{array}\right]\right] \rightarrow \rho^{\prime}\left(t_{\mathrm{CP}}^{\left[1^{\prime}-2^{\prime}\right]}\right)=\left[\left[\begin{array}{c}
0 \\
0 \\
R / 2 \\
R / 2 \\
-R / 2 \\
-R / 2
\end{array}\right]\right] \\
& \left.\rho^{\prime}\left(t_{\text {spinlock }}\right)=\left[\left[\begin{array}{c}
-R / 2 \\
R / 2 \\
R / 2 \\
R / 2 \\
-R / 2 \\
-R / 2
\end{array}\right]\right] \rightarrow \rho^{\prime}\left(t_{\mathrm{CP}}^{\left[2^{\prime}-6^{\prime}\right]}\right)=\left[\begin{array}{c}
-R / 2 \\
0 \\
R / 2 \\
R / 2 \\
-R / 2 \\
0
\end{array}\right]\right] \\
& \left.\rho^{\prime}\left(t_{\text {spinlock }}\right)=\left[\left[\begin{array}{c}
-R / 2 \\
R / 2 \\
R / 2 \\
R / 2 \\
-R / 2 \\
-R / 2
\end{array}\right]\right] \rightarrow \rho^{\prime}\left(t_{\mathrm{CP}}^{\left[3^{\prime}-5^{\prime}\right]}\right)=\left[\begin{array}{c}
-R / 2 \\
R / 2 \\
0 \\
R / 2 \\
0 \\
-R / 2
\end{array}\right]\right] \\
& \left.\rho^{\prime}\left(t_{\text {spinlock }}\right)=\left[\left[\begin{array}{c}
-R / 2 \\
R / 2 \\
R / 2 \\
R / 2 \\
-R / 2 \\
-R / 2
\end{array}\right]\right] \rightarrow \rho^{\prime}\left(t_{\mathrm{CP}}^{\left[4^{\prime}-5^{\prime}\right]}\right)=\left[\begin{array}{c}
-R / 2 \\
R / 2 \\
R / 2 \\
0 \\
0 \\
-R / 2
\end{array}\right]\right]
\end{aligned}
$$


At next, the density matrix after the CP step is transferred back in the doubly rotating frame via the inverse transformation of Eq. 5.23:

$$
\begin{aligned}
\rho\left(t_{\mathrm{CP}}^{\left[i^{\prime}-j^{\prime}\right]}\right) & =U_{x}^{-1}\left(\theta_{\gamma}, \theta_{\beta}, \theta_{\alpha}\right)\left\{\rho^{\prime}\left(t_{\mathrm{CP}}^{\left[i^{\prime}-j^{\prime}\right]}\right)\right\} U_{x}\left(\theta_{\gamma}, \theta_{\beta}, \theta_{\alpha}\right) \\
& =U_{x}^{-1}(0, \pi / 2, \pi)\left\{\rho^{\prime}\left(t_{\mathrm{CP}}^{\left[i^{\prime}-j^{\prime}\right]}\right)\right\} U_{x}(0, \pi / 2, \pi)
\end{aligned}
$$

and after letting the off-diagonal elements decay for time $\tau>T_{2 e}$, we obtain

$$
\begin{aligned}
\rho\left(t_{\mathrm{CP}}^{\left[1^{\prime}-2^{\prime}\right]}\right)= & {\left[\left[\begin{array}{c}
0 \\
R / 4 \\
R / 2 \\
-R / 2 \\
R / 4 \\
-R / 2
\end{array}\right], \rho\left(t_{\mathrm{CP}}^{\left[2^{\prime}-6^{\prime}\right]}\right)=\left[\left[\begin{array}{c}
R / 2 \\
R / 4 \\
0 \\
-R / 2 \\
R / 4 \\
-R / 2
\end{array}\right]\right],\right.} \\
\rho\left(t_{\mathrm{CP}}^{\left[3^{\prime}-5^{\prime}\right]}\right)= & {\left.\left[\left[\begin{array}{c}
R / 2 \\
-R / 4 \\
R / 2 \\
-R / 2 \\
-R / 4 \\
0
\end{array}\right]\right], \rho\left(t_{\mathrm{CP}}^{\left[4^{\prime}-5^{\prime}\right]}\right)=\left[\begin{array}{c}
R / 2 \\
-R / 4 \\
R / 2 \\
0 \\
-R / 4 \\
-R / 2
\end{array}\right]\right] }
\end{aligned}
$$

From here the CP-ENDOR signal intensities are for example calculated according to

$$
\begin{aligned}
S_{\text {echo, } \beta}^{\mathrm{NMR}}{ }^{1-2}\left(\mathrm{CP}\left[1^{\prime}-2^{\prime}\right]\right) & \sim \operatorname{Tr}\left\{\left(U_{\pi}^{1-2}\right) \rho\left(t_{\mathrm{CP}}^{\left[1^{\prime}-2^{\prime}\right]}\right)\left(U_{\pi}^{1-2}\right)^{-1} I_{z}^{2-5}\right\} \\
& \sim \operatorname{Tr}\left\{\rho\left(t_{\mathrm{CP}}^{\left[1^{\prime}-2^{\prime}\right]}\right)\left(U_{\pi}^{1-2}\right)^{-1} I_{z}^{2-5}\left(U_{\pi}^{1-2}\right)\right\} \\
& \sim \operatorname{Tr}\left\{\rho\left(t_{\mathrm{CP}}^{\left[1^{\prime}-2^{\prime}\right]}\right) I_{z}^{1-5}\right\}
\end{aligned}
$$

and

$$
\begin{aligned}
& S_{\text {echo, } \beta}^{\mathrm{NMR} 2-3}\left(\mathrm{CP}\left[1^{\prime}-2^{\prime}\right]\right) \sim \operatorname{Tr}\left\{\rho\left(t_{\mathrm{CP}}^{\left[1^{\prime}-2^{\prime}\right]}\right) I_{z}^{3-5}\right\} \\
& S_{\text {echo, } \beta}^{\mathrm{NMR}} 4^{-5}\left(\mathrm{CP}\left[1^{\prime}-2^{\prime}\right]\right) \sim \operatorname{Tr}\left\{\rho\left(t_{\mathrm{CP}}^{\left[1^{\prime}-2^{\prime}\right]}\right) I_{z}^{2-4}\right\} \\
& S_{\text {echo, } \beta}^{\mathrm{NMR} 5-6}\left(\mathrm{CP}\left[1^{\prime}-2^{\prime}\right]\right) \sim \operatorname{Tr}\left\{\rho\left(t_{\mathrm{CP}}^{\left[1^{\prime}-2^{\prime}\right]}\right) I_{z}^{2-6}\right\}
\end{aligned}
$$

The calculated CP-ENDOR intensities are listed in Table 5.4. 


\section{E. Control CP-ENDOR spectrum of ${ }^{2} \mathrm{H}-\mathrm{BDPA}$}

The ENDOR spectra presented in Chapter 6 consistently showed higher intensity of the lines at $\nu_{\mathrm{n}}^{2 \mathrm{H}}+A / 2 \pi$ compared to $\nu_{\mathrm{n}}^{2 \mathrm{H}}-A / 2 \pi$. In this particular case, this general asymmetry of ENDOR lines most likely arises from hardware imperfection such as non-linear rf amplifier output power. This hypothesis is supported by the experiments shown in Fig. E2, which were done at an early stage of this thesis. Here, ENDOR lines appear symmetric with equal intensities.

\section{A) $2 D$ WOP CP-ENDOR}

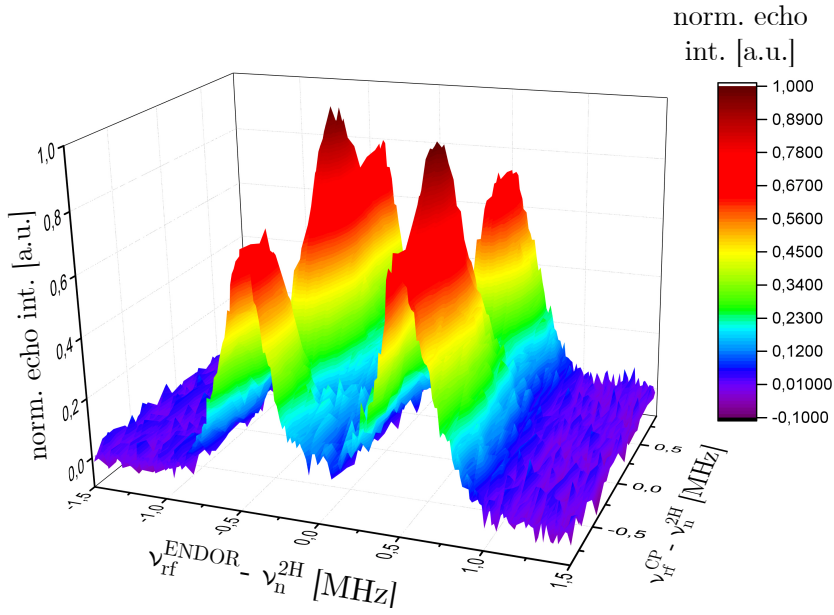

\section{B) ENDOR comparison}

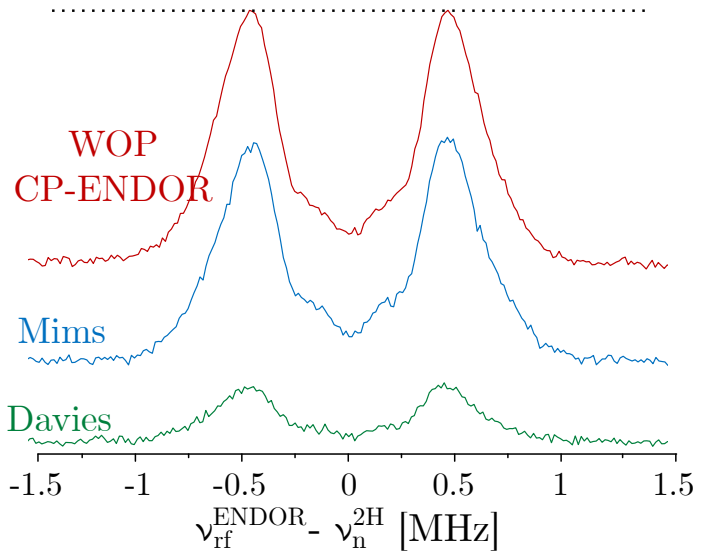

Figure E2: Mims, Davies and WOP CP-ENDOR control experiments of ${ }^{2}$ H-BDPA. A) 2D WOP CP-ENDOR landscape and contour plot. B) Mims, Davies and summed WOP CP-ENDOR spectra with the same acquisition time. Experimental parameters: $T=80 \mathrm{~K}, \nu_{\mathrm{EPR}}=94 \mathrm{GHz}, t_{\pi, \mathrm{rf}}=38 \mu \mathrm{s}$, shot repetition time $=25 \mathrm{~ms}, 1$ shot $/$ point, random rf acquisition, 1000 scans $(2.5 \mathrm{~h}$ averaging time); Davies: $t_{\pi, \text { prep }}=400 \mathrm{~ns}, t_{\pi / 2, \operatorname{det}}=20 \mathrm{~ns} ;$ Mims: $t_{\pi / 2, \mathrm{mw}}=20 \mathrm{~ns} ; C P$ : $t_{\pi / 2, \operatorname{det}}=200 \mathrm{~ns}, \omega_{1 e} / 2 \pi=1.25 \mathrm{MHz}, t_{\mathrm{CP}}=200 \mu \mathrm{s}, \Delta \omega_{\mathrm{n}} / 2 \pi=-0.9 \mathrm{MHz}$ to $+0.9 \mathrm{MHz}$ (20 steps). 


\section{F. PulseSPEL script for ${ }^{2} \mathrm{H}-\mathrm{BDPA}$ 2D CP-ENDOR experiments}

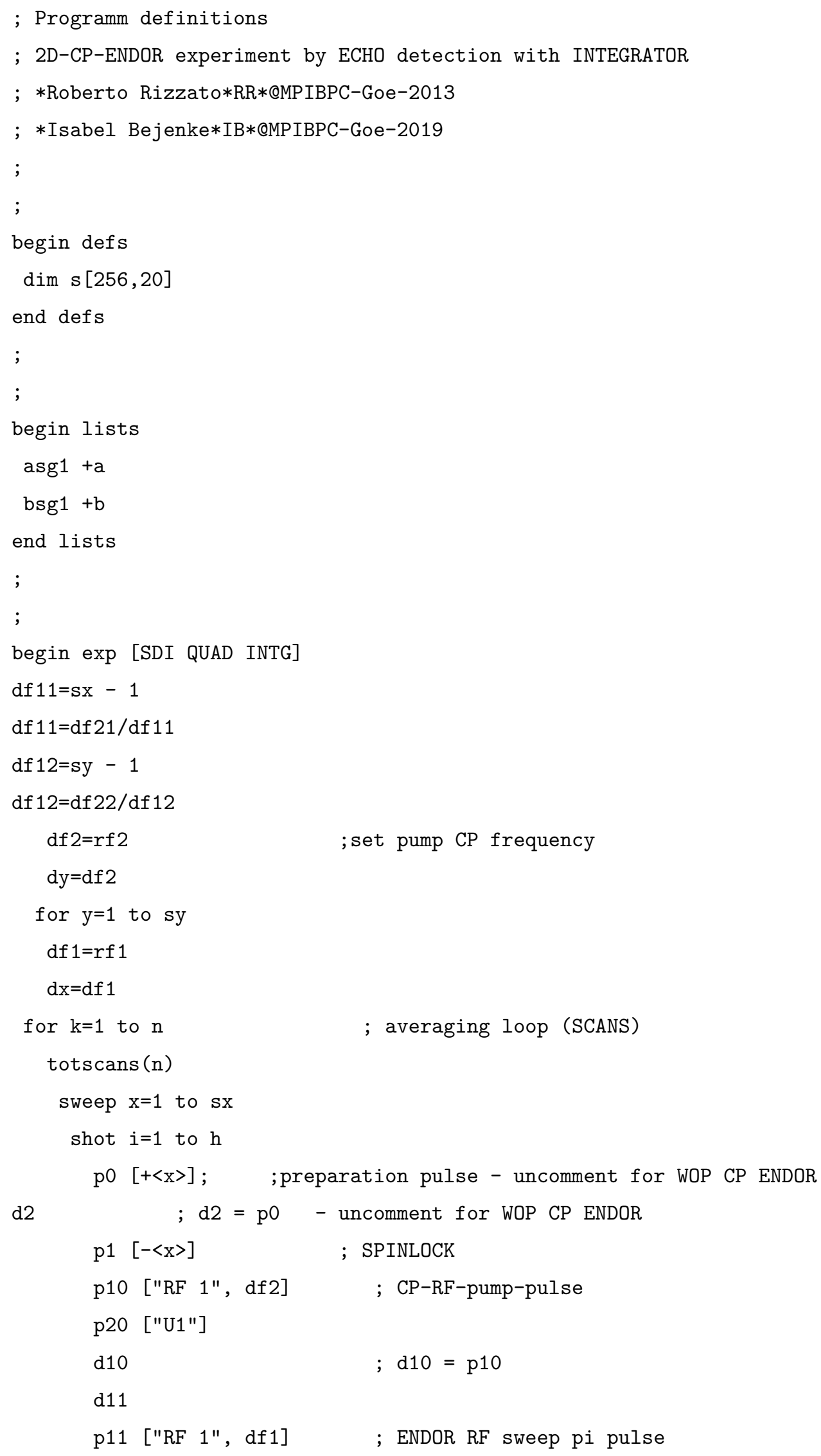




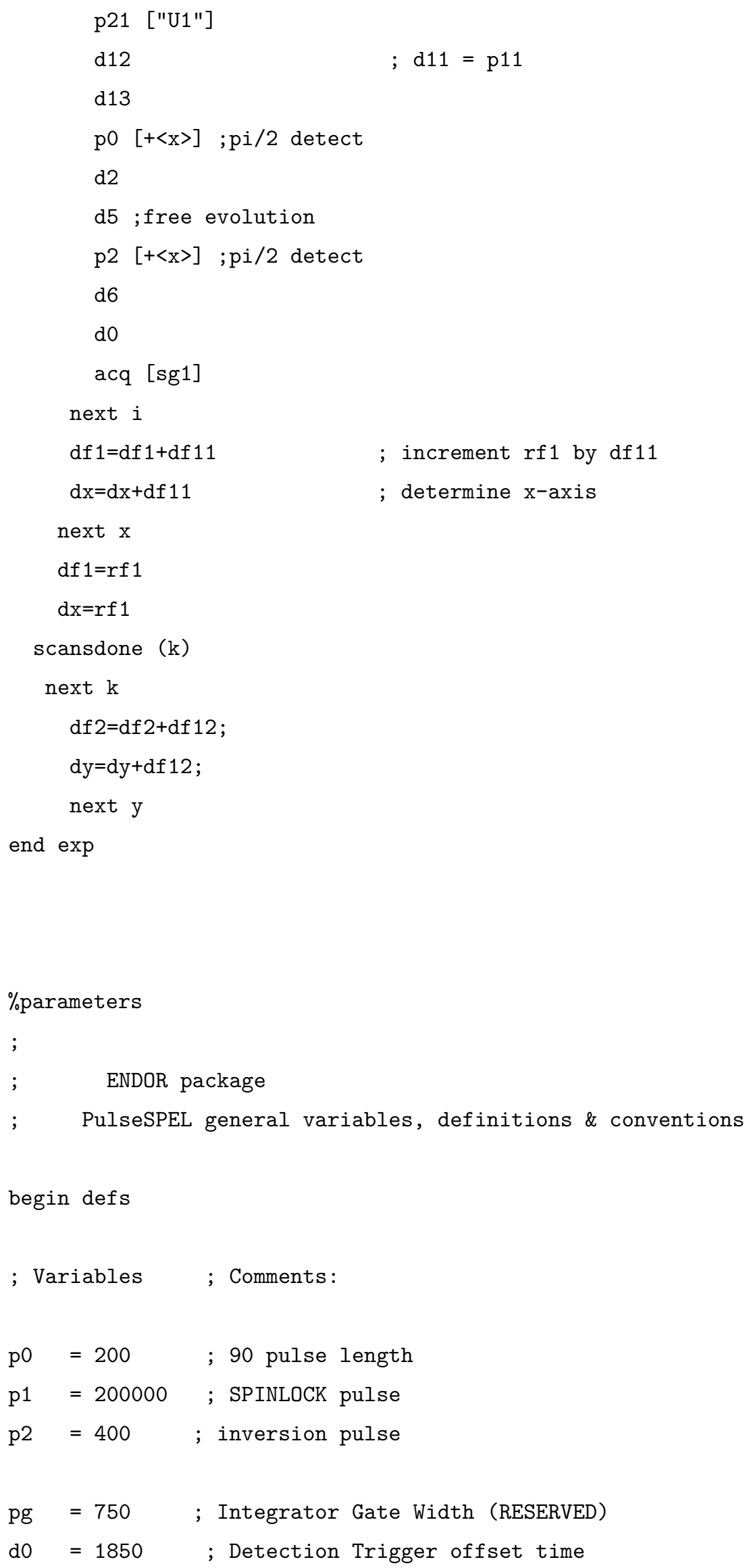




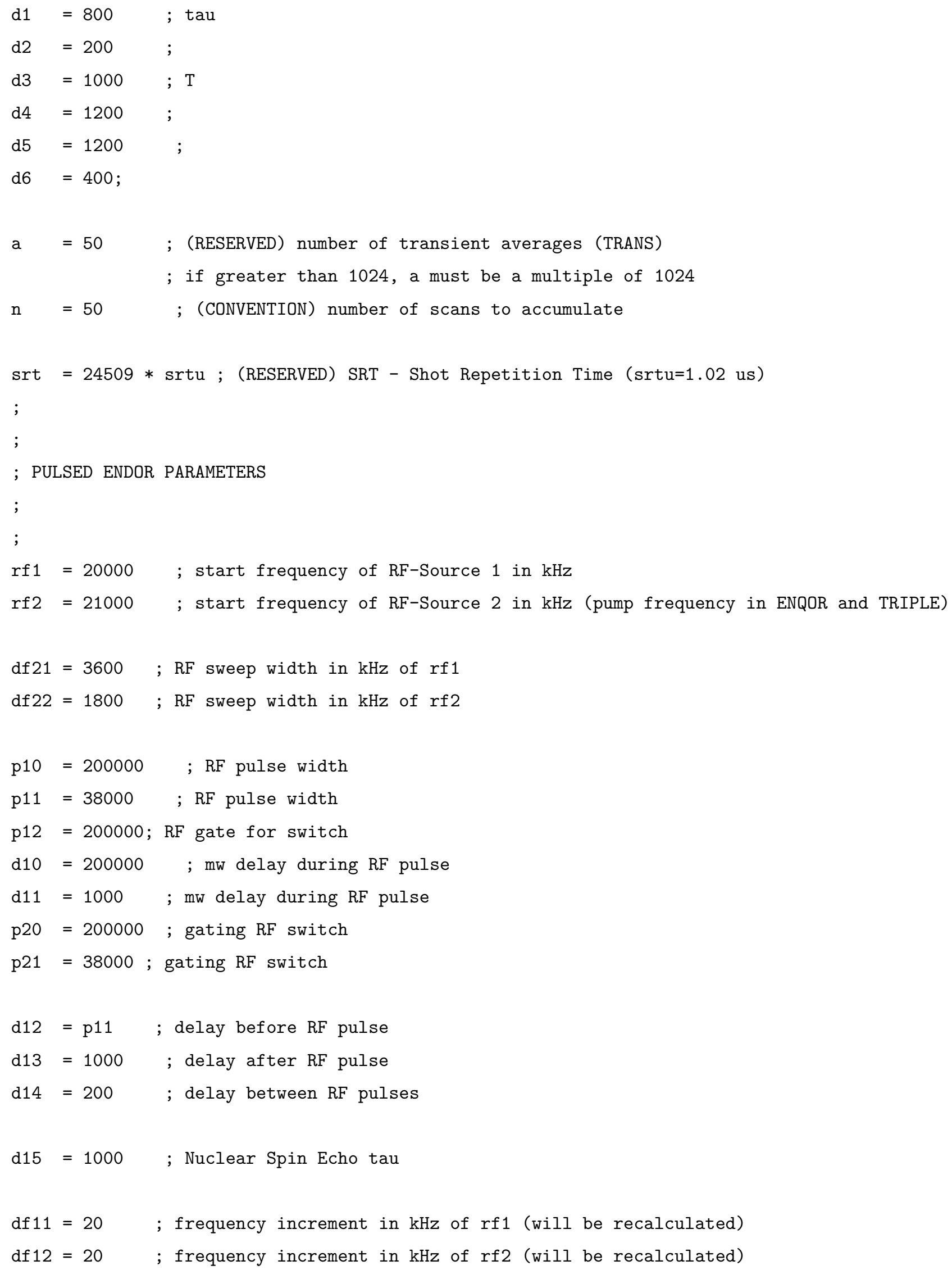


end defs

G. Calculation script for plotting of analytically derived excitation profile

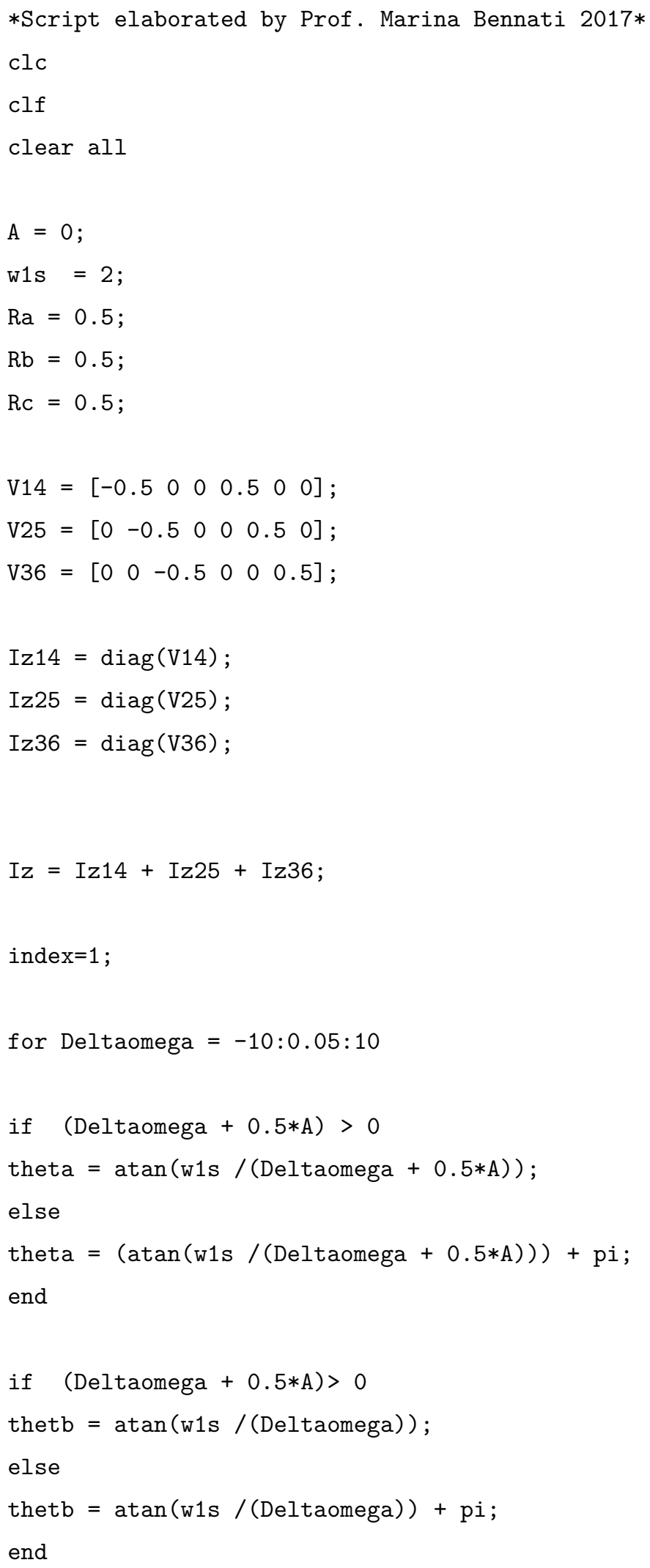


if (Deltaomega $+0.5 * \mathrm{~A})>0$

thetc $=\operatorname{atan}(w 1 \mathrm{~s} /($ Deltaomega $-0.5 * \mathrm{~A}))$;

else

thetc $=(\operatorname{atan}(w 1 s /($ Deltaomega $-0.5 * A)))+p i ;$

end

omegaalpha $=\operatorname{sqrt}\left((\text { Deltaomega }+A)^{\wedge} 2+w_{1} \mathrm{~s}^{\wedge} 2\right)$;

omegagamma $=\operatorname{sqrt}\left((\text { Deltaomega }-A)^{\wedge} 2+w_{1} s^{\wedge} 2\right)$;

omegabeta $=\operatorname{sqrt}\left((\text { Deltaomega })^{\wedge} 2+w 1 s^{\wedge} 2\right) ;$

\%density matrix at end of spinlock, no CP match, neglecting off diagonal elements rho $=-$ Ra $* \cos ($ theta $) * I z 14-R b * \cos ($ thetb $) * I z 25-R c * \cos ($ thetc $) * I z 36$;

Secho14 $=\operatorname{abs}(2 * \operatorname{trace}(\operatorname{rho} * \operatorname{Iz} 14))$;

Secho25 $=\operatorname{abs}(2 * \operatorname{trace}($ rho*Iz25));

Secho36 $=\operatorname{abs}(2 * \operatorname{trace}(\operatorname{rho} * \operatorname{Iz} 36))$;

hndl = plot (Deltaomega, Secho25, 'r.' $)$;

set (hndl, 'MarkerSize', 10);

hold on

Delta $($ index $)=$ Deltaomega

Signal (index) = Secho14;

index $=$ index +1 ;

end 


\section{H. eNCP spectrum of $\mathrm{ND}_{2} \mathrm{Y}_{731^{\bullet}}$}

The eNCP spectrum of $\mathrm{ND}_{2} \mathrm{Y}_{731}$ (Fig. $\mathrm{H} 3$ ) was performed in order to experimentally determine the matching condition range. The eNCP experiment was performed with the same $\omega_{1 \mathrm{e}} / 2 \pi$ and spinlock pulse length as for the CP-ENDOR experiments in Fig. 7.1. The matching condition range was identified to $\Delta \omega_{\mathrm{n}}^{\mathrm{CP}} / 2 \pi \approx \pm 2.3 \mathrm{MHz}$

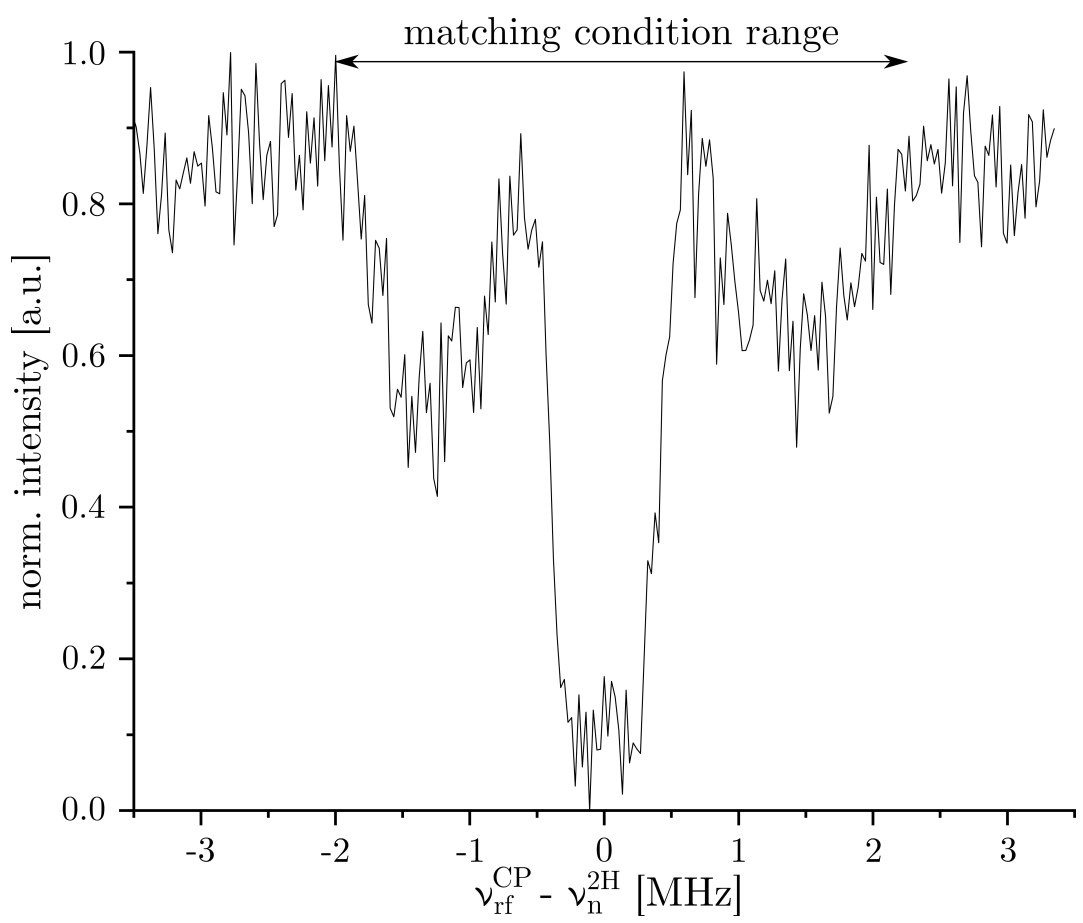

Figure H3: eNCP spectrum of $\mathrm{ND}_{\mathbf{2}} \mathbf{Y}_{\mathbf{7 3 1}} \bullet^{\circ}$. Exp. conditions: $T=10 \mathrm{~K}$, $\nu_{\mathrm{EPR}}=94 \mathrm{GHz}$, shot repetition time $=100 \mathrm{~ms}, 1 \mathrm{shot} /$ point, random $\mathrm{rf}$ acquisition; $t_{\pi / 2}=200 \mathrm{~ns}, \omega_{1 \mathrm{e}} / 2 \pi=1.25 \mathrm{MHz}, t_{\mathrm{CP}}=200 \mu \mathrm{s}, 1400$ scans. 



\section{Bibliography}

[1] Zänker, P.-P.; Jeschke, G.; Goldfarb, D. Distance measurements between paramagnetic centers and a planar object by matrix Mims electron nuclear double resonance. The Journal of Chemical Physics 2005, 122, 0245151-02451511.

[2] Schiemann, O.; Prisner, T. F. Long-range distance determinations in biomacromolecules by EPR spectroscopy. Quarterly Reviews of Biophysics 2007, 40, $1-53$.

[3] Feher, G. Observation of Nuclear Magnetic Resonances via the Electron Spin Resonance Line. Physical Review 1956, 103, PR, 834-835.

[4] Mims, W. B. Pulsed Endor Experiments. Proceedings of the Royal Society of London. Series A, Mathematical and Physical Sciences 1965, 283, 452-457.

[5] Davies, E. R. A new pulse endor technique. Physics Letters A 1974, 47, 1-2.

[6] Bennati, M.; Prisner, T. New Developments in high field EPR with applications in structural biology. Reports on Progress in Physics 2005, 68, 411-448.

[7] Tkach, I.; Bejenke, I.; Hecker, F.; Kehl, A.; Kasanmascheff, M.; Gromov, I.; Prisecaru, I.; Höfer, P.; Hiller, M.; Bennati, M. 1H High Field Electron-Nuclear Double Resonance Spectroscopy at $263 \mathrm{GHz} / 9.4$ Tesla. Journal of Magnetic Resonance 2019, 303, 17-27.

[8] Savitsky, A.; Möbius, K. High-field EPR. Photosynthesis Research 2009, 102, 311-333.

[9] Lubitz, W.; Ogata, H.; Rüdiger, O.; Reijerse, E. Hydrogenases. Chemical Reviews 2014, 114, 4081-4148. 
[10] Rao, G.; Tao, L.; Suess, D. L. M.; Britt, R. D. A [4Fe-4S]-Fe(CO)(CN)-l-cysteine intermediate is the first organometallic precursor in $[\mathrm{FeFe}]$ hydrogenase $\mathrm{H}$-cluster bioassembly. Nature Chemistry 2018, 10, 555-560.

[11] Cox, N.; Retegan, M.; Neese, F.; Pantazis, D. A.; Boussac, A.; Lubitz, W. Electronic structure of the oxygen-evolving complex in photosystem II prior to O-O bond formation. Science 2014, 345, 804-808.

[12] Van Dam, P. J.; Willems, J.-P.; Schmidt, P. P.; Pötsch, S.; Barra, A.-L.; Hagen, W. R.; Hoffman, B. M.; Andersson, K. K.; Gräslund, A. High-Frequency EPR and Pulsed Q-Band ENDOR Studies on the Origin of the Hydrogen Bond in Tyrosyl Radicals of Ribonucleotide Reductase R2 Proteins from Mouse and Herpes Simplex Virus Type 1. Journal of the American Chemical Society 1998, 120, 5080-5085.

[13] Bennati, M.; Farrar, C.; Bryant, J.; Inati, S.; Weis, V.; Gerfen, G.; Riggs-Gelasco, P.; Stubbe, J.; Griffin, R. Pulsed ENDOR at 140 GHz. Journal of Magnetic Resonance 1999, 132, 232-243.

[14] Srinivas, V.; Lebrette, H.; Lundin, D.; Kutin, Y.; Sahlin, M.; Lerche, M.; Eirich, J.; M. M. Branca, R.; Cox, N.; Sjöberg, B.-M.; Högbom, M. Metal-free ribonucleotide reduction powered by a DOPA radical in Mycoplasma pathogens. 2018, 563, 416-420.

[15] Blaesi, E. J.; Palowitch, G. M.; Hu, K.; Kim, A. J.; Rose, H. R.; Alapati, R.; Lougee, M. G.; Kim, H. J.; Taguchi, A. T.; Tan, K. O.; Laremore, T. N.; Griffin, R. G.; Krebs, C.; Matthews, M. L.; Silakov, A.; Bollinger, J. M.; Allen, B. D.; Boal, A. K. Metal-free class Ie ribonucleotide reductase from pathogens initiates catalysis with a tyrosine-derived dihydroxyphenylalanine radical. Proceedings of the National Academy of Sciences 2018, 115, 10022-10027.

[16] Argirević, T.; Riplinger, C.; Stubbe, J.; Neese, F.; Bennati, M. ENDOR Spectroscopy and DFT Calculations: Evidence for the Hydrogen-Bond Network Within $\alpha 2$ in the PCET of E. coli Ribonucleotide Reductase. Journal of the American Chemical Society 2012, 134, 17661-17670.

[17] Nick, T. U.; Lee, W.; Koßmann, S.; Neese, F.; Stubbe, J.; Bennati, M. Hydrogen Bond Network between Amino Acid Radical Intermediates on the Proton-Coupled Electron Transfer Pathway of E. coli $\alpha 2$ Ribonucleotide Reductase. Journal of the American Chemical Society 2015, 137, 289-298. 
[18] Kasanmascheff, M.; Lee, W.; Nick, T. U.; Stubbe, J.; Bennati, M. Radical transfer in E. coli ribonucleotide reductase: a $\mathrm{NH}_{2} \mathrm{Y}_{731} / \mathrm{R}_{411} \mathrm{~A}-\alpha$ mutant unmasks a new conformation of the pathway residue 731. Chemical Science 2016, 7, 2170-2178.

[19] Nick, T. U.; Ravichandran, K. R.; Stubbe, J.; Kasanmascheff, M.; Bennati, M. Spectroscopic Evidence for a $\mathrm{H}$ Bond Network at $\mathrm{Y}_{356}$ Located at the Subunit Interface of Active E. coli Ribonucleotide Reductase. Biochemistry 2017, 56, 3647-3656.

[20] Chandrashekar, T. K.; O’Malley, P. J.; Rodriguez, I.; Babcock, G. T. Endor characterization and $\mathrm{D}_{2} \mathrm{O}$ exchange in the $Z .^{+} / D .^{+}$radical in photosystem II. Photosynthesis Research 1986, 10, 423-429.

[21] Schleicher, E.; Wenzel, R.; Ahmad, M.; Batschauer, A.; Essen, L.-O.; Hitomi, K.; Getzoff, E. D.; Bittl, R.; Weber, S.; Okafuji, A. The Electronic State of Flavoproteins: Investigations with Proton Electron-Nuclear Double Resonance. Applied magnetic resonance 2010, 37, 339-352.

[22] Davydov, R.; Dawson, J. H.; Perera, R.; Hoffman, B. M. The Use of Deuterated Camphor as a Substrate in $1 \mathrm{H}$ ENDOR Studies of Hydroxylation by Cryoreduced Oxy P450cam Provides New Evidence of the Involvement of Compound I. Biochemistry 2013, 52, 667-671.

[23] Force, D. A.; Randall, D. W.; Britt, R. D.; Tang, X.-S.; Diner, B. A. 2H ESE-ENDOR study of hydrogen bonding to the tyrosine radicals YD.bul. and YZ.bul. of photosystem II. Journal of the American Chemical Society 1995, 117, 12643-12644.

[24] Shanmugam, M.; Xue, G.; Que, L.; Hoffman, B. M. 1H-ENDOR Evidence for a Hydrogen-Bonding Interaction That Modulates the Reactivity of a Nonheme FeIV=O Unit. Inorganic Chemistry 2012, 51, 10080-10082.

[25] Rizzato, R.; Kaminker, I.; Vega, S.; Bennati, M. Cross-polarisation edited ENDOR. Molecular Physics 2013, 111, 2809-2823.

[26] Rizzato, R.; Bennati, M. Enhanced sensitivity of electron-nuclear double resonance (ENDOR) by cross polarisation and relaxation. Physical Chemistry Chemical Physics 2014, 16, 7681-7685.

[27] Rizzato, R.; Bennati, M. Cross-Polarization Electron-Nuclear Double Resonance Spectroscopy. ChemPhysChem 2015, 16, 3769-3773. 
[28] Abragam, A., The principles of nuclear magnetism; Clarendon Press ; Oxford University Press: Oxford [Oxfordshire]; New York, 1961, (c)2011.

[29] Schweiger, A.; Jeschke, G., Principles of pulse electron paramagnetic resonance; Oxford University Press: Oxford, UK; New York, 2001.

[30] Poole, C. P.; Farach, H. A., Theory of magnetic resonance; Wiley: New York, 1987.

[31] Brustolon, M., Electron Paramagnetic Resonance: A Practitioner's Toolkit; Wiley: Hoboken, NJ, 2009.

[32] Stoll, S.; Goldfarb, D. EPR Interactions - Nuclear Quadrupole Couplings. In: eMagRes 2017, 495-510.

[33] Bennati, M.; Murphy, D. M. Electron Paramagnetic Resonance Spectra in the Solid State. In: Electron Paramagnetic Resonance: A Practitioner's Toolkit 2008, 195-250.

[34] Bennati, M. EPR Interactions - Hyperfine Couplings. In: eMagRes 2017, 6, $271-281$.

[35] Fermi, E. Über die magnetischen Momente der Atomkerne. Zeitschrift fur Physik 1930, 60, 320-333.

[36] Bloch, F.; Hansen, W. W.; Packard, M., Nuclear induction, 1946.

[37] Neumann, J. v. Wahrscheinlichkeitstheoretischer Aufbau der Quantenmechanik. Nachrichten von der Gesellschaft der Wissenschaften zu Göttingen, MathematischPhysikalische Klasse 1927, 1927, 245-272.

[38] Slichter, C. P., Principles of magnetic resonance; Springer-Verlag: Berlin; New York, 1996.

[39] Levitt, M. H., Spin dynamics: basics of nuclear magnetic resonance; Wiley: Chichester, 2015.

[40] Gemperle, C.; Schweiger, A. Pulsed ENDOR methodology. 1991, 91, 1481-1505.

[41] Sørensen, O. W.; Eich, G. W.; Levitt, M. H.; Bodenhausen, G.; Ernst, R. R. Product operator formalism for the description of NMR pulse experiments. Progress in Nuclear Magnetic Resonance Spectroscopy 1984, 16, 163-192.

[42] Tait, C. E.; Stoll, S. ENDOR with band-selective shaped inversion pulses. Journal of Magnetic Resonance 2017, 277, 36-44. 
[43] Harmer, J. R. Hyperfine Spectroscopy - ENDOR. In: eMagRes 2016, 14931514.

[44] Stoll, S.; Shafaat, H. S.; Krzystek, J.; Ozarowski, A.; Tauber, M. J.; Kim, J. E.; Britt, R. D. Hydrogen Bonding of Tryptophan Radicals Revealed by EPR at 700 GHz. Journal of the American Chemical Society 2011, 133, 18098-18101.

[45] Schnegg, A.; Dubinskii, A. A.; Fuchs, M. R.; Grishin, Y. A.; Kirilina, E. P.; Lubitz, W.; Plato, M.; Savitsky, A.; Möbius, K. High-field EPR, ENDOR and ELDOR on bacterial photosynthetic reaction centers. Applied Magnetic Resonance 2007, 31, 59-98.

[46] Hertel, M. M.; Denysenkov, V. P.; Bennati, M.; Prisner, T. F. Pulsed 180-GHz EPR/ENDOR/PELDOR spectroscopy. Magnetic resonance in chemistry: MRC. 2005, 43, 248-255.

[47] Murphy, D. M.; Farley, R. D. Principles and applications of ENDOR spectroscopy for structure determination in solution and disordered matrices. Chemical Society Reviews 2006, 35, 249-268.

[48] Bennati, M.; Tkach, I.; Turke, M. T. Dynamic nuclear polarization in liquids. In: Electron Paramagnetic Resonance 2011, 22, 155-182.

[49] Epel, B.; Pöppl, A.; Manikandan, P.; Vega, S.; Goldfarb, D. Effect of spin relaxation on ENDOR spectra at high fields and low T. Journal of Magnetic Resonance 2001, 148, 388-397.

[50] Tyryshkin, A. M.; Morton, J. J. L.; Ardavan, A.; Lyon, S. A. Davies electronnuclear double resonance revisited: Enhanced sensitivity and nuclear spin relaxation. The Journal of Chemical Physics 2006, 124, 2345081-2345087.

[51] Morton, J. J. L.; Lees, N. S.; Hoffman, B. M.; Stoll, S. Nuclear relaxation effects in Davies ENDOR variants. Journal of Magnetic Resonance 2008, 191, 315-321.

[52] Rizzato, R. Development of cross-polarization ENDOR spectroscopy. GeorgAugust-Universität Göttingen 2015, PhD Thesis.

[53] Epel, B.; Arieli, D.; Baute, D.; Goldfarb, D. Improving W-band pulsed ENDOR sensitivity-random acquisition and pulsed special TRIPLE. Journal of Magnetic Resonance 2003, 164, 78-83. 
[54] Mehring, M.; Höfer, P.; Grupp, A. Pulsed electron nuclear double and triple resonance schemes. Berichte der Bunsengesellschaft für physikalische Chemie 1987, 91, 1132-1137.

[55] Hartmann, S.; Hahn, E. L. Nuclear double resonance in the rotating frame. Physical Review 1962, 128, 2042-2053.

[56] Pines, A.; Gibby, M. G.; Waugh, J. S. Protonenhanced NMR of dilute spins in solids. The Journal of Chemical Physics 1973, 59, 569-590.

[57] Tan, K. O.; Yang, C.; Mathies, G.; Griffin, R. G.; Weber, R. T.; Mathies, G. Time-optimized pulsed dynamic nuclear polarization. Science Advances 2019, 5.

[58] Weis, V.; Bennati, M.; Rosay, M.; Griffin, R. G. Solid effect in the electron spin dressed state: A new approach for dynamic nuclear polarization. Journal of Chemical Physics 2000, 113, 6795-6802.

[59] Weis, V.; Griffin, R. G. Electron nuclear cross polarization. Solid State Nuclear Magnetic Resonance 2006, 29, 105-117.

[60] Reichard, P. From RNA to DNA, why so many ribonucleotide reductases? Science 1993, 260, 1773-1777.

[61] Thelander, L.; Reichard, P. Reduction of Ribonucleotides. Annual Review of Biochemistry 1979, 48, 133-158.

[62] Stubbe, J. Ribonucleotide reductases: the link between an RNA and a DNA world? Current opinion in structural biology. 2000, 10, 731-736.

[63] Minnihan, E. C.; Ando, N.; Brignole, E. J.; Olshansky, L.; Chittuluru, J.; Asturias, F. J.; Drennan, C. L.; Nocera, D. G.; Stubbe, J. Generation of a stable, aminotyrosyl radical-induced $\alpha 2 \beta 2$ complex of Escherichia coli class Ia ribonucleotide reductase. Proceedings of the National Academy of Sciences 2013, $110,3835-3840$.

[64] Stubbe, J. Ribonucleotide reductases in the twenty-first century. Proceedings of the National Academy of Sciences of the United States of America 1998, 95, 2723-2724.

[65] Reichard, P.; Ehrenberg, A. Ribonucleotide reductase-a radical enzyme. Science Science 1983, 221, 514-519.

[66] Nordlund, P.; Sjöberg, B. M.; Eklund, H. Three-dimensional structure of the free radical protein of ribonucleotide reductase. Nature 1990, 345, 593-8. 
[67] Larsson, A.; Sjöberg, B. Identification of the stable free radical tyrosine residue in ribonucleotide reductase. The EMBO Journal 1986, 5, 2037-2040.

[68] Un, S.; Gerez, C.; Elleingand, E.; Fontecave, M. Sensitivity of Tyrosyl Radical g-Values to Changes in Protein Structure: A High-Field EPR Study of Mutants of Ribonucleotide Reductase. Journal of the American Chemical Society 2001, 123, 3048-3054.

[69] Bennati, M.; Robblee, J. H.; Mugnaini, V.; Stubbe, J.; Freed, J. H.; Borbat, P. EPR Distance Measurements Support a Model for Long-Range Radical Initiation in E. coli Ribonucleotide Reductase. Journal of the American Chemical Society 2005, 127, 15014-15015.

[70] Stubbe, J.; Seyedsayamdost, M. R. Discovery of a New Class I Ribonucleotide Reductase with an Essential DOPA Radical and NO Metal as an Initiator of Long-Range Radical Transfer. Biochemistry 2019, 58, 435-437.

[71] Jordan, A.; Reichard, P. Ribonucleotide Reductases. Annual Review of Biochemistry 1998, 67, 71-98.

[72] Nordlund, P.; Reichard, P. Ribonucleotide Reductases. Annual Review of Biochemistry 2006, 75, 681-706.

[73] Graslund, A.; Sahlin, M. Electron Paramagnetic Resonance and Nuclear Magnetic Resonance Studies of Class I Ribonucleotide Reductase. Annual Review of Biophysics and Biomolecular Structure 1996, 25, 259-286.

[74] Stubbe, J.; Nocera, D. G.; Yee, C. S.; Chang, M. C. Y. Radical Initiation in the Class I Ribonucleotide Reductase: Long-Range Proton-Coupled Electron Transfer? Chemical Reviews 2003, 103, 2167-2202.

[75] Thelander, L. Physicochemical characterization of ribonucleoside diphosphate reductase from Escherichia coli. The Journal of biological chemistry 1973, 248, 4591-601.

[76] Stubbe, J.; van der Donk, W. A. Chemical Reviews 1998, 98, 705-762.

[77] Lenz, R.; Giese, B. Studies on the Mechanism of Ribonucleotide Reductases. Journal of the American Chemical Society 1997, 119, 2784-2794.

[78] Uhlin, U.; Eklund, H. Structure of ribonucleotide reductase protein R1. Nature 1994, 370, 533-539. 
[79] Seyedsayamdost, M. R.; Xie, J.; Chan, C. T. Y.; Schultz, P. G.; Stubbe, J. Site-Specific Insertion of 3-Aminotyrosine into Subunit $\alpha 2$ of E. coli Ribonucleotide Reductase: Direct Evidence for Involvement of $\mathrm{Y}_{730}$ and $\mathrm{Y}_{731}$ in Radical Propagation. Journal of the American Chemical Society 2007, 129, 15060-15071.

[80] Ando, N.; Brignole, E. J.; Zimanyi, C. M.; Funk, M. A.; Yokoyama, K.; Asturias, F. J.; Stubbe, J.; Drennan, C. L. Structural interconversions modulate activity of Escherichia coli ribonucleotide reductase. Proceedings of the National Academy of Sciences 2011, 108, 21046-21051.

[81] Ravichandran, K. R.; Minnihan, E. C.; Wei, Y.; Nocera, D. G.; Stubbe, J. Reverse Electron Transfer Completes the Catalytic Cycle in a 2,3,5-TrifluorotyrosineSubstituted Ribonucleotide Reductase. Journal of the American Chemical Society 2015, 13\%, 14387-14395.

[82] Climent, I.; Sjoeberg, B. M.; Huang, C. Y. Site-directed mutagenesis and deletion of the carboxyl terminus of Escherichia coli ribonucleotide reductase protein R2. Effects on catalytic activity and subunit interaction. Biochemistry Biochemistry 1992, 31, 4801-4807.

[83] Migliore, A.; Polizzi, N. F.; Therien, M. J.; Beratan, D. N. Biochemistry and Theory of Proton-Coupled Electron Transfer. Chemical Reviews 2014, 114, $3381-3465$.

[84] Ge, J.; Yu, G.; Ator, M. A.; Stubbe, J. Pre-Steady-State and Steady-State Kinetic Analysis of E. coli Class I Ribonucleotide Reductase. Biochemistry 2003, 42, 10071-10083.

[85] Minnihan, E. C.; Seyedsayamdost, M. R.; Uhlin, U.; Stubbe, J. Kinetics of Radical Intermediate Formation and Deoxynucleotide Production in 3-AminotyrosineSubstituted Escherichia coli Ribonucleotide Reductases. Journal of the American Chemical Society 2011, 133, 9430-9440.

[86] Lee, W.; Kasanmascheff, M.; Huynh, M.; Quartararo, A.; Costentin, C.; Bejenke, I.; Nocera, D. G.; Bennati, M.; Tommos, C.; Stubbe, J. Properties of Site-Specifically Incorporated 3-Aminotyrosine in Proteins To Study RedoxActive Tyrosines: Escherichia coli Ribonucleotide Reductase as a Paradigm. Biochemistry 2018, 57, 3402-3415. 
[87] Hosseinzadeh, P.; Lu, Y. Design and fine-tuning redox potentials of metalloproteins involved in electron transfer in bioenergetics. Biochimica et Biophysica Acta (BBA) - Bioenergetics 2016, 1857, 557-581.

[88] Sanderud, A.; Sagstuen, E.; Itagaki, Y.; Lund, A. EPR and ENDOR Studies of Deuteron Hyperfine and Quadrupole Coupling in $\bullet \mathrm{CD}(\mathrm{COOD})_{2}$ : Experimental and Theoretical Estimates of Electric Field Gradients from an $\alpha$-Carbon. The Journal of Physical Chemistry A 2000, 104, 6372-6379.

[89] Do, C.; Hatfield, J.; Patel, S.; Vasudevan, D.; Tirla, C.; Mills, N. S. Dications of Benzylidenefluorene and Diphenylmethylidene Fluorene: The Relationship between Magnetic and Energetic Measures of Antiaromaticity. The Journal of Organic Chemistry 2011, 76, 181-187.

[90] Kuhn, R.; Neugebauer, A. Über substituierte Bis-biphenylen-allyl-Radikale. Monatshefte für Chemie und verwandte Teile anderer Wissenschaften 1964, 95, $3-23$.

[91] Hausser, K. H. Hyperfeinstruktur und Relaxationsmechanismus der Elektronenresonanz in Lösung. Zeitschrift für Naturforschung A 1959, 14.

[92] Salowe, S. P.; Stubbe, J. Cloning, overproduction, and purification of the B2 subunit of ribonucleoside-diphosphate reductase. Journal of Bacteriology 1986, $165,363-366$.

[93] Vega, S.; Pines, A. Operator formalism for double quantum NMR. The Journal of Chemical Physics 1977, 66, 5624-5644.

[94] Vega, S. Fictitious spin 1/2 operator formalism for multiple quantum NMR. The Journal of Chemical Physics 1978, 68, 5518-5527.

[95] Dalton, L. R.; Kwiram, A. L. ENDOR Studies in Molecular Crystals. II. Computer Analysis of the Polycrystalline ENDOR Spectra of Low Symmetry Materials. The Journal of Chemical Physics 1972, 5\%, 1132-1145.

[96] Stoll, S.; Schweiger, A. EasySpin, a comprehensive software package for spectral simulation and analysis in EPR. Journal of Magnetic Resonance 2006, 178, 42-55.

[97] Weis, V.; Bennati, M.; Rosay, M.; Bryant, J. A.; Griffin, R. G. High-field DNP and ENDOR with a novel multiple-frequency resonance structure. Journal of Magnetic Resonance 1999, 140, 293-299. 
[98] Klauder, J. R.; Anderson, P. W. Spectral Diffusion Decay in Spin Resonance Experiments. Physical Review 1962, 125, PR, 912-932.

[99] Dzuba, S. A.; Kawamori, A. Selective hole burning in EPR: Spectral diffusion and dipolar broadening. Concepts in Magnetic Resonance 1996, 8, 49-61.

[100] Schosseler, P.; Wacker, T.; Schweiger, A. Pulsed ELDOR detected NMR. Chemical Physics Letters 1994, 224, 319-324.

[101] Cox, N.; Nalepa, A.; Lubitz, W.; Savitsky, A. ELDOR-detected NMR: A general and robust method for electron-nuclear hyperfine spectroscopy? Journal of Magnetic Resonance 2017, 280, 63-78.

[102] Jeschke, G. Generation and transfer of coherence in electron-nuclear spin systems by non-ideal microwave pulses. Molecular Physics 1996, 88, 355-383.

[103] Brunner, H.; Fritsch, R.; Hausser, K. Cross polarization in electron nuclear double resonance by satisfying the Hartmann-Hahn condition. Zeitschrift für Naturforschung 1987, 42a, 1456-1457.

[104] Henstra, A.; Dirksen, P.; Schmidt, J.; Wenckebach, W. T. Nuclear spin orientation via electron spin locking (NOVEL). Journal of Magnetic Resonance (1969) 1988, 77, 389-393.

[105] Reinhard, M. Kreuzpolarisationseditierte Elektronen-Kern-DoppelresonanzSpektroskopie an deuterierten Proteinradikalen. Georg-August-Universität Göttingen 2016, Bachelor Thesis.

[106] Seyedsayamdost, M. R.; Argirević, T.; Minnihan, E. C.; Stubbe, J.; Bennati, M. Structural Examination of the Transient 3-Aminotyrosyl Radical on the PCET Pathway of E. coli Ribonucleotide Reductase by Multifrequency EPR Spectroscopy. Journal of the American Chemical Society 2009, 131, 1572915738.

[107] Motion, C. L.; Cassidy, S. L.; Cruickshank, P. A. S.; Hunter, R. I.; Bolton, D. R.; El Mkami, H.; Van Doorslaer, S.; Lovett, J. E.; Smith, G. M. The use of composite pulses for improving DEER signal at $94 \mathrm{GHz}$. Journal of Magnetic Resonance 2017, 278, 122-133.

[108] Spindler, P. E.; Zhang, Y.; Endeward, B.; Gershernzon, N.; Skinner, T. E.; Glaser, S. J.; Prisner, T. F. Shaped optimal control pulses for increased excitation bandwidth in EPR. Journal of Magnetic Resonance 2012, 218, 49-58. 
[109] Spindler, P. E.; Glaser, S. J.; Skinner, T. E.; Prisner, T. F. Broadband Inversion PELDOR Spectroscopy with Partially Adiabatic Shaped Pulses. Angewandte Chemie International Edition 2013, 52, 3425-3429. 



\section{Acknowledgments}

Firstly, I would like to thank my advisor Prof. Dr. Marina Bennati for all her contributions of knowledge, ideas, and funding to make my $\mathrm{PhD}$ experience productive and fruitful. I appreciate her good balance between guidance and freedom which allowed to do this work. I may have been lost in the doubly-rotating tilted frame sometimes, however, her encouragement and advice have guided me through, so that the past four years were challenging, but not a maze.

My gratitude also goes to the members of my thesis committee Prof. Dr. Christian Griesinger and Prof. Dr. Martin Suhm for making my committee meetings a pleasant experience.

In addition, I want to thank Prof. Dr. Steffen Glaser and Dr. Robert Zeier (TU Munich) for their immense knowledge, motivation and patience during our collaborative research, which made me think outside of the experimentalists box.

In addition to my advisors, I want to thank all members of the EPR spectroscopy group, past and current, for the wonderful atmosphere of collegiality and support in scientific matters and beyond. In particular Roberto Rizzato for setting the basis for my research. A significant part of this work would not have been possible if you would not have done the spin gymnastics with me during the first year of my PhD. A very special word of thanks goes to Igor Tkach. His scientific expertise and technical skills paired with his patient attitude were indispensable to overcome countless small and large instrumentation issues.

Furthermore, I would like to express my gratitude to Karin Halbmair, Müge Kasanmascheff and Tomas Orlando for their friendship and support through my entire time in the group. Your energy and positivity is contagious and has motivated me even during tough times. Further, I want to thank my fellow PhD students Fabian Hecker and 
Marcel Levien. I will always remember the scientific discussions and fun-time we had together at conferences, as well as the evenings dedicated to board games. Markus Hiller, Andreas Meyer, Francesco Torricella, Gabriele Valora, Nikolay Enkin and Thomas Nick for, besides many other things, always being helpful. My gratitude goes to Brigitta Angerstein for her practical help especially with sample preparation, and Monika Frömel for assistance in apparently never-ending administrative tasks. I am thankful for my motivated and talented student co-workers including Annemarie Kehl, Maik Reinhardt and Bastian Zimmer during their respective Bachelor's theses.

Many thanks go to my friends for always making me smile and lots of joyful moments that enrich my life.

Most of all, I want to thank my parents. You have encouraged me to go my own way in life and you are always there with overwhelming support, experience, and love. This work is dedicated to both of you. 


\section{Publications}

Bejenke, I.; Zeier, R.; Rizzato,R.; Glaser, S. J.; Bennati, M. Cross-polarization edited ENDOR for spin-1 deuterium nuclei. to be submitted.

Tkach, I.; Bejenke, I.; Hecker, F.; Kehl, A.; Kasanmascheff, M.; Gromov, I.; Prisecaru, I.; Höfer, P.; Hiller, M.; Bennati, M. 1H High Field Electron-Nuclear Double Resonance Spectroscopy at $263 \mathrm{GHz} / 9.4$ Tesla. Journal of Magnetic Resonance 2019, 303, 17-27.

Lee, W.; Kasanmascheff, M.; Huynh, M.; Quartartaro, A.; Constentin, C.; Bejenke, I.; Nocera, D. G.; Bennati, M.; Tommos, C.; Stubbe, J. Properties of Site-Specifically Incorporated 3-Aminotyrosine in Proteins to Study Redox-Active Tyrosines: E. coli Ribonucleotide Reductase as a Paradigm. Biochemistry 2018, 57 (24), 3402-3415.

Frank, M.; Ahrens, J.; Bejenke, I.; Krick, M.; Schwarzer, D.; Clever, G.H. Light-Induced Charge Separation in Densely Packed Donor-Acceptor Coordination Cages. Journal of the American Chemical Society 2016, 138 (26), 8279-8287. 
